\title{
Matter waves II \\ Mesoscopic transport of coherent bosonic matter waves
}

\author{
Peter Schlagheck \\ Université de Liège
}




\title{
Matter waves II Mesoscopic transport of coherent bosonic matter waves
}

\author{
Peter Schlagheck \\ Université de Liège
}




\section{Outline}

- Bose-Einstein condensation

- guided atom lasers

- quasi-stationary (?) transport of interacting bosonic matter waves: mean-field description and beyond

- breakdown of Anderson localization in 1D disorder potentials

- coherent backscattering in 2D disorder potentials

- summary and FAQs 


\section{Bose-Einstein condensation}

Noninteracting atoms in harmonic traps: $T>T_{c}$

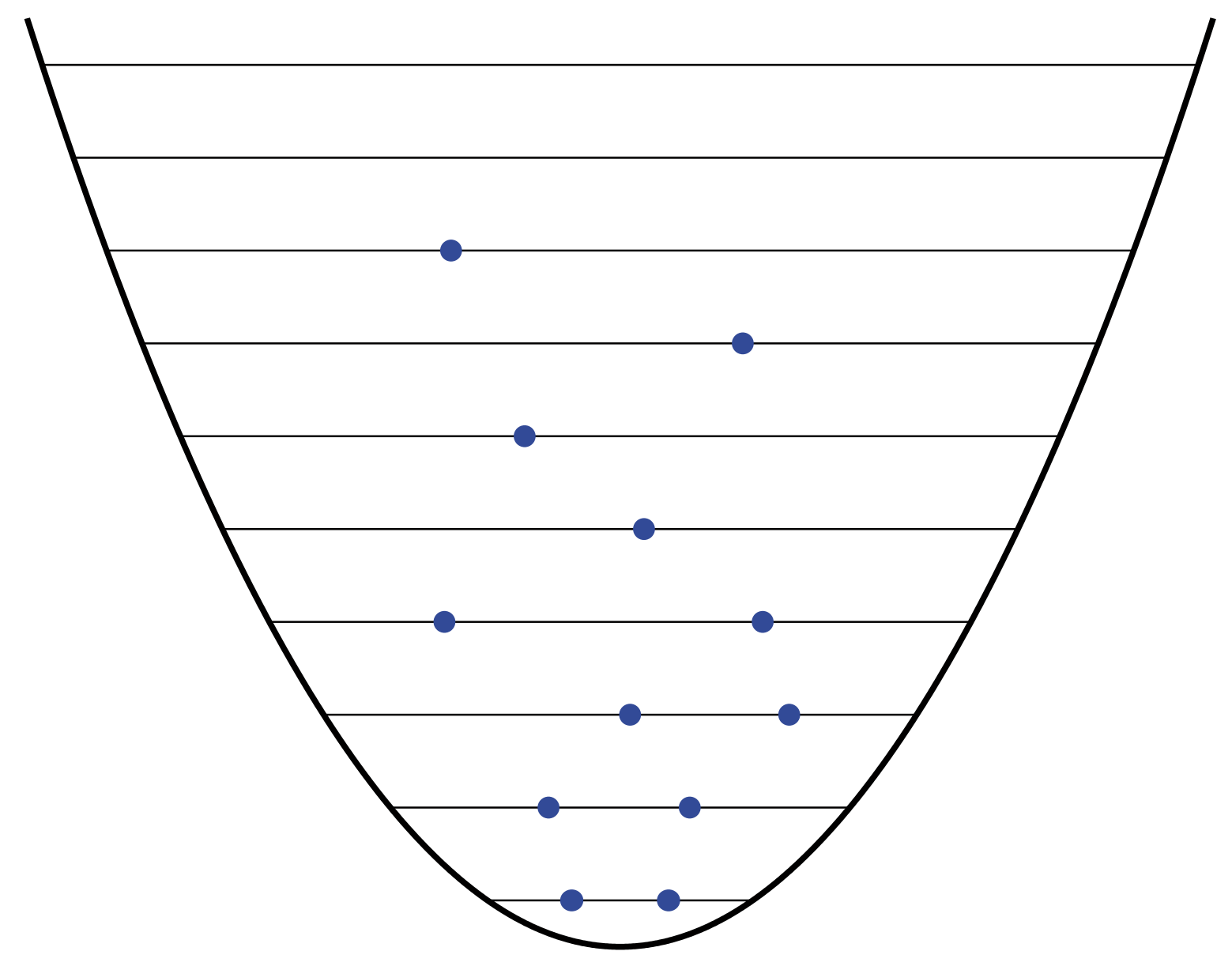




\section{Bose-Einstein condensation}

Noninteracting atoms in harmonic traps: $T \lesssim T_{c}$

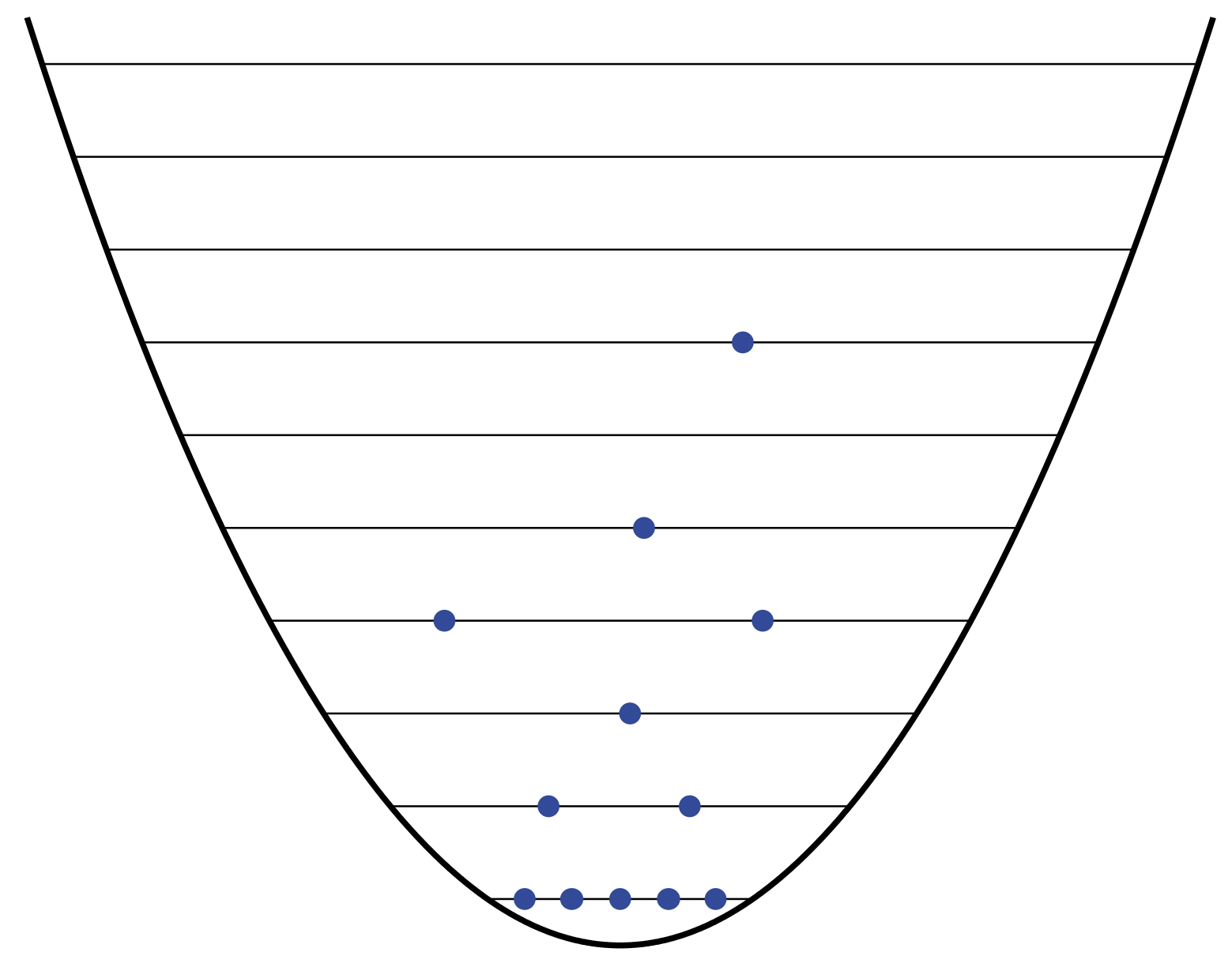




\section{Bose-Einstein condensation}

Noninteracting atoms in harmonic traps: $T \ll T_{c}$

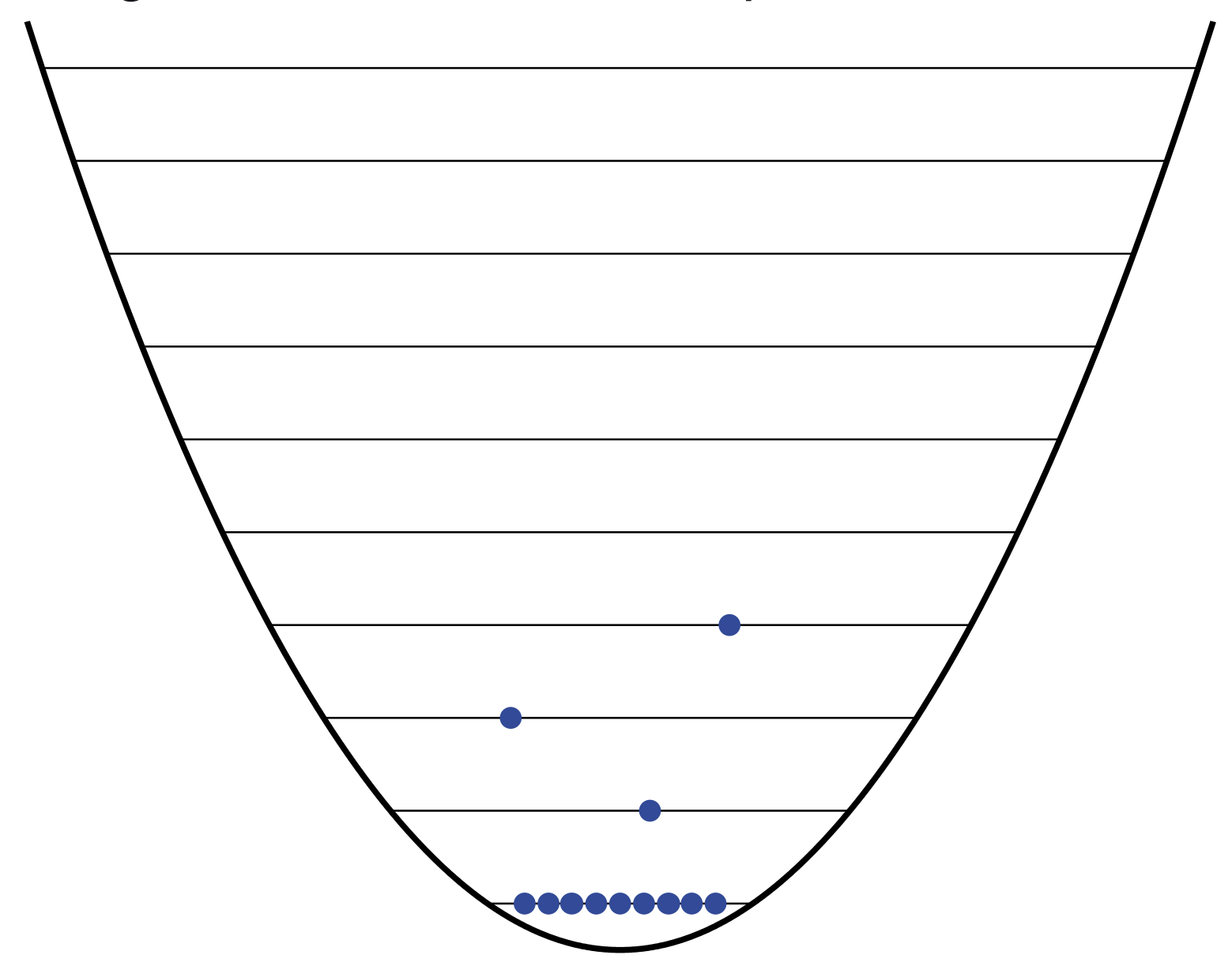

$\longrightarrow$ ground state population: $N_{0} / N=\left(1-T / T_{c}\right)^{3}$ 


\section{Bose-Einstein condensation}

Noninteracting atoms in harmonic traps: $T=0$

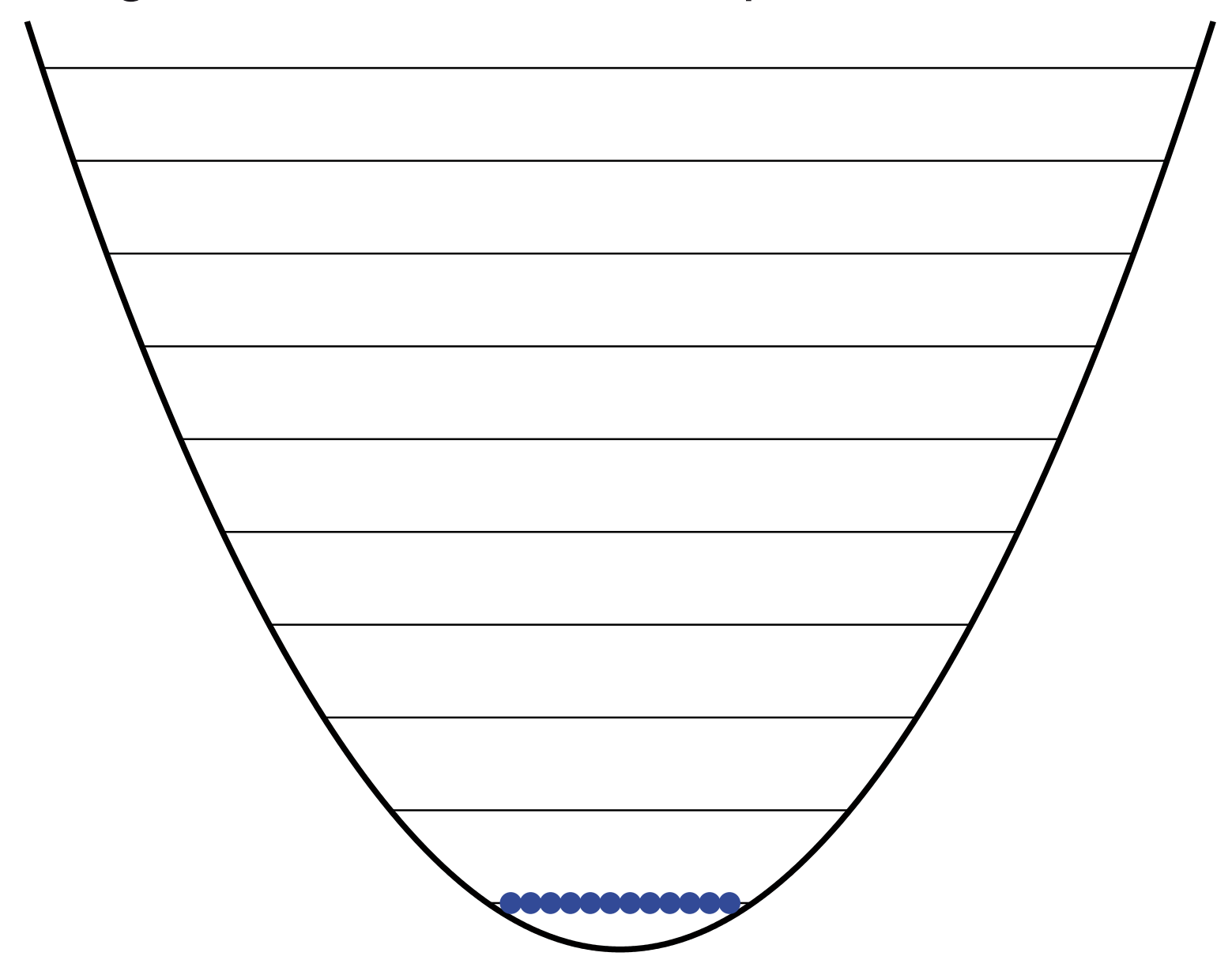

$\longrightarrow$ ground state population: $N_{0} / N=\left(1-T / T_{c}\right)^{3}$ 


\section{Atom-atom interaction}

short-range interaction potential $U\left(\mathbf{r}_{1}-\mathbf{r}_{2}\right)$

with spatial extent $\sim \mathrm{nm} \ll$ mean inter-atomic distance

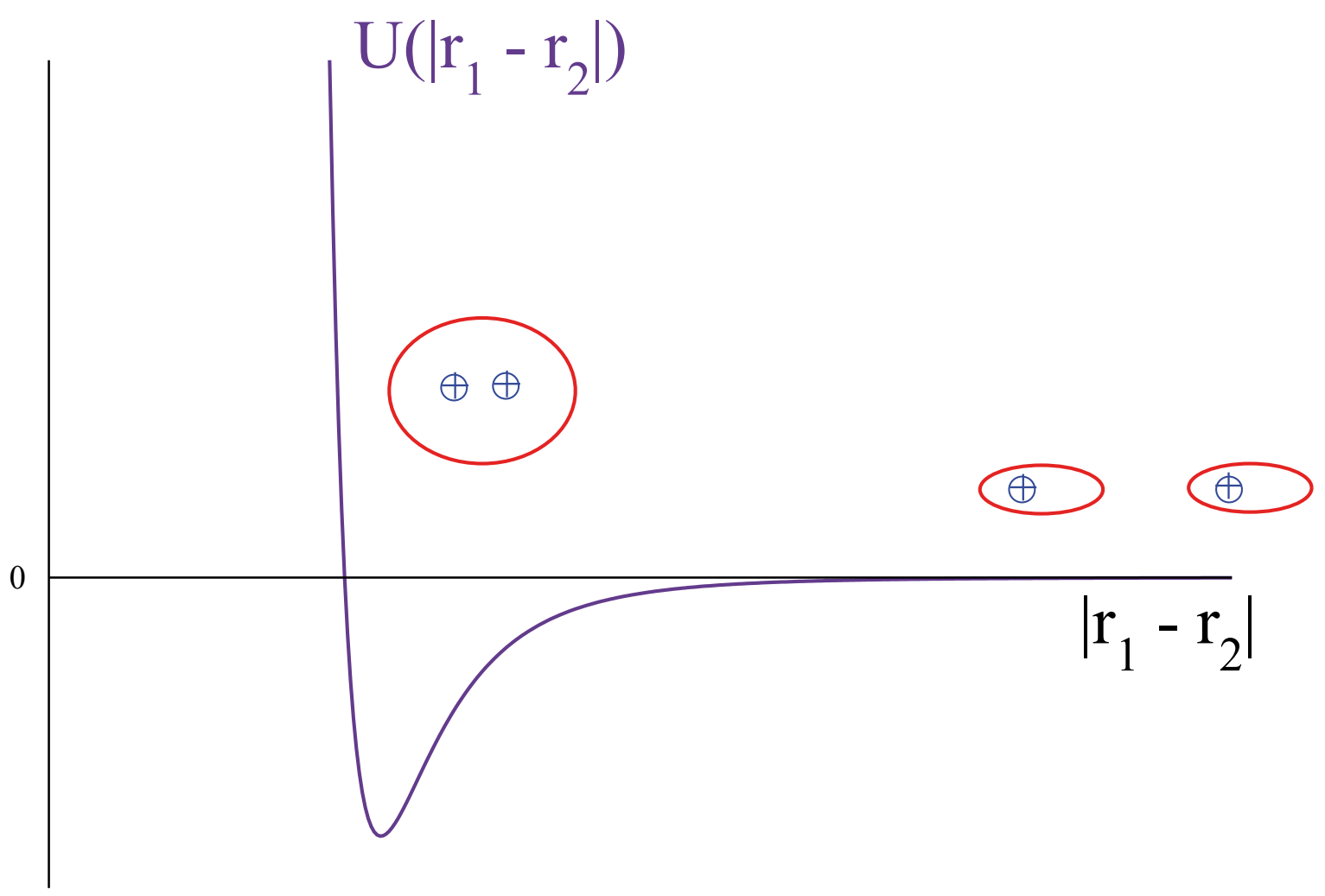




\section{Atom-atom interaction}

$\longrightarrow$ short-range interaction potential $U\left(\mathbf{r}_{1}-\mathbf{r}_{2}\right)$

with spatial extent $\sim \mathrm{nm} \ll$ mean inter-atomic distance

$\longrightarrow$ replace it by a contact interaction (pseudo-)potential

$$
U\left(\mathbf{r}_{1}-\mathbf{r}_{2}\right)=U_{0} \delta\left(\mathbf{r}_{1}-\mathbf{r}_{2}\right)
$$

that reproduces the asymptotic scattering properties of two atoms:

$$
U_{0}=\frac{4 \pi \hbar^{2} a_{s}}{m}
$$

with $a_{s}$ the $s$-wave scattering length of the atomic species 


\section{Atom-atom interaction}

$\longrightarrow$ short-range interaction potential $U\left(\mathbf{r}_{1}-\mathbf{r}_{2}\right)$

with spatial extent $\sim \mathrm{nm} \ll$ mean inter-atomic distance

$\longrightarrow$ replace it by a contact interaction (pseudo-)potential

$$
\left.\left.(U \psi)\right|_{\mathbf{r}_{1}, \mathbf{r}_{2}} \equiv U_{0} \delta\left(\mathbf{r}_{1}-\mathbf{r}_{2}\right) \frac{\partial}{\partial r}[r \psi]\right|_{r=\left|\mathbf{r}_{1}-\mathbf{r}_{2}\right|}
$$

that reproduces the asymptotic scattering properties of two atoms:

$$
U_{0}=\frac{4 \pi \hbar^{2} a_{s}}{m}
$$

with $a_{s}$ the $s$-wave scattering length of the atomic species 


\section{Atom-atom interaction}

$\longrightarrow$ short-range interaction potential $U\left(\mathbf{r}_{1}-\mathbf{r}_{2}\right)$

with spatial extent $\sim \mathrm{nm} \ll$ mean inter-atomic distance

$\longrightarrow$ replace it by a contact interaction (pseudo-)potential

$$
\left.\left.(U \psi)\right|_{\mathbf{r}_{1}, \mathbf{r}_{2}} \equiv U_{0} \delta\left(\mathbf{r}_{1}-\mathbf{r}_{2}\right) \frac{\partial}{\partial r}[r \psi]\right|_{r=\left|\mathbf{r}_{1}-\mathbf{r}_{2}\right|}
$$

that reproduces the asymptotic scattering properties of two atoms:

$$
U_{0}=\frac{4 \pi \hbar^{2} a_{s}}{m}
$$

with $a_{s}$ the $s$-wave scattering length of the atomic species

$\longrightarrow$ stable condensate for $a_{s}>0$ (effective repulsion) 


\section{$B E C$ with atom-atom interaction}

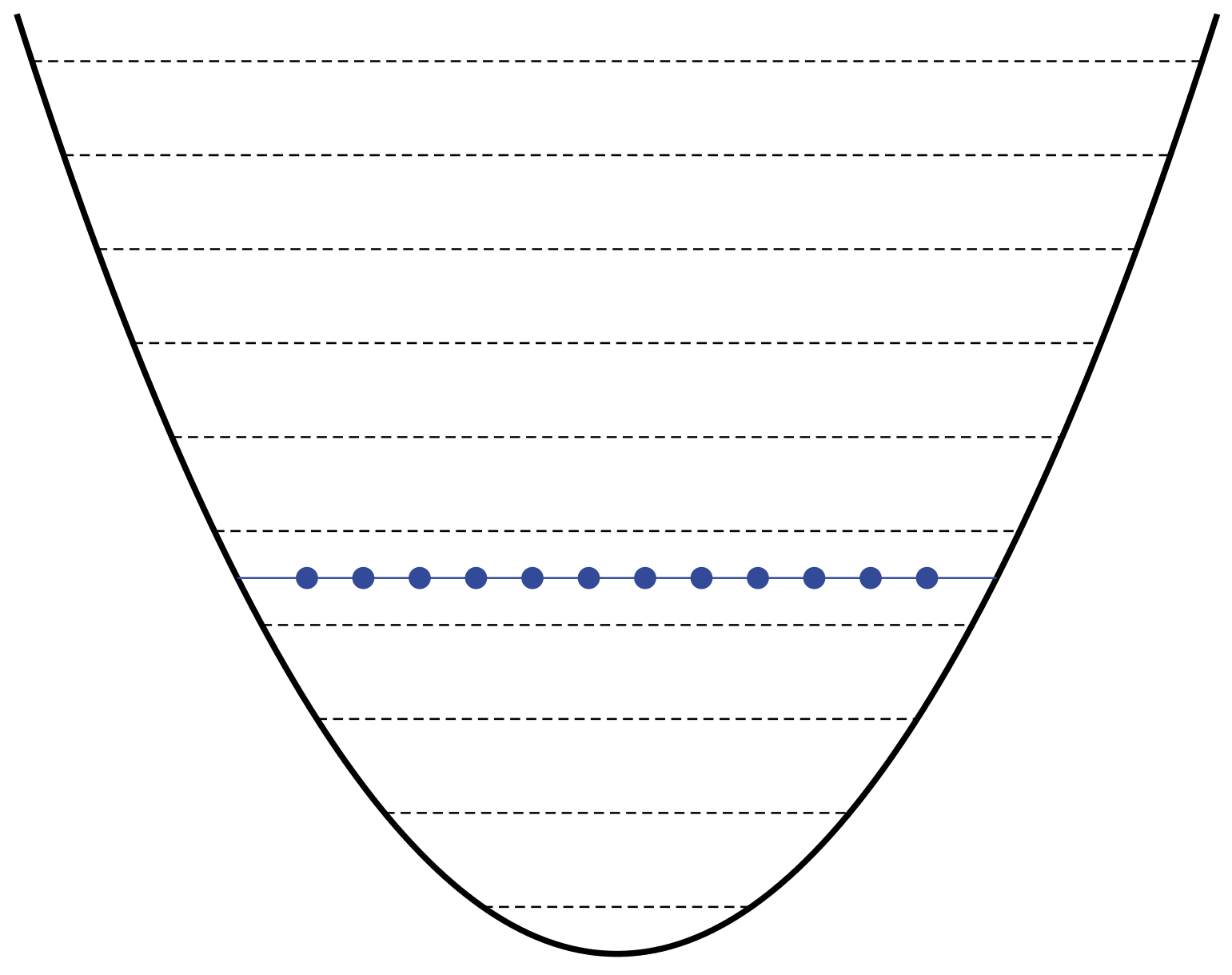




\section{BEC with atom-atom interaction}

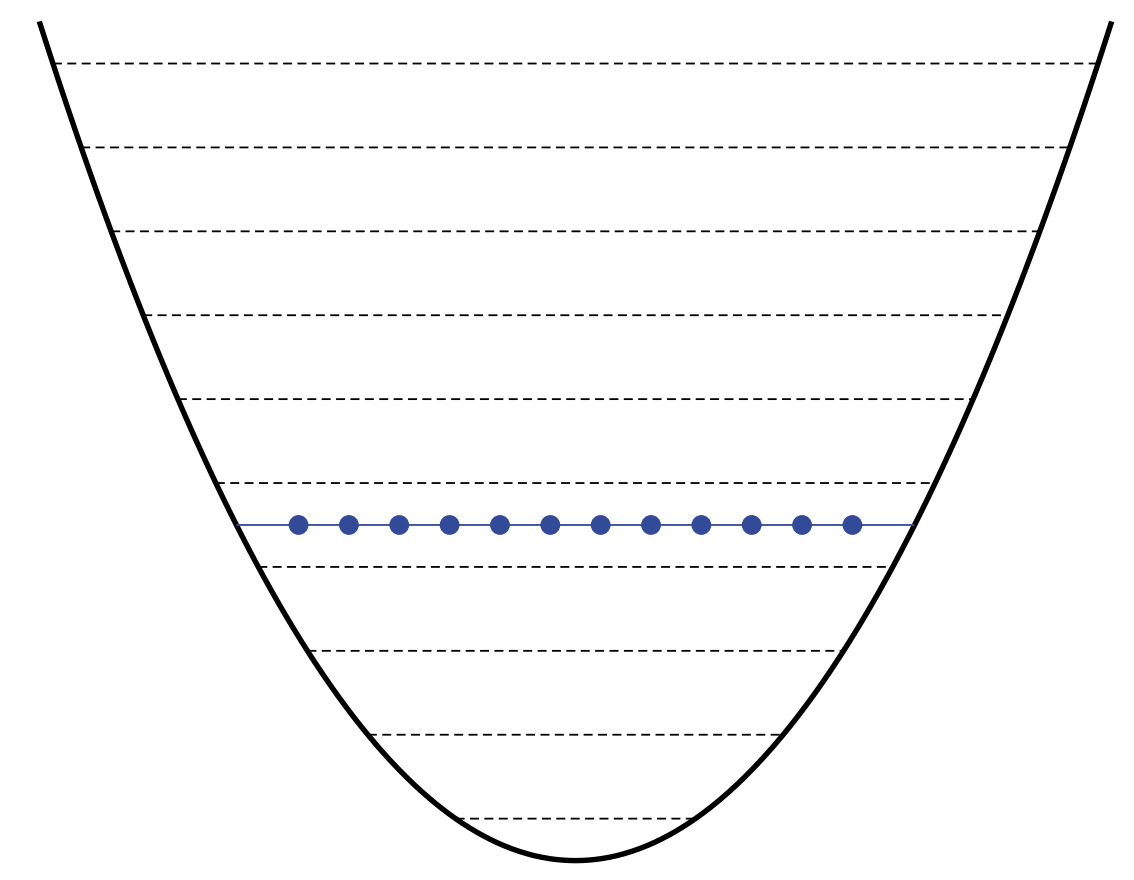

$\rightarrow$ modified condensate wavefunction $\psi$ and chemical potential $\mu$

$$
\left(-\frac{\hbar^{2}}{2 m} \frac{\partial^{2}}{\partial \mathbf{r}^{2}}+V_{\text {trap }}(\mathbf{r})+U_{0}|\psi(\mathbf{r})|^{2}\right) \psi(\mathbf{r})=\mu \psi(\mathbf{r})
$$

$\longrightarrow$ Gross-Pitaevskii equation 


\section{BEC with atom-atom interaction}

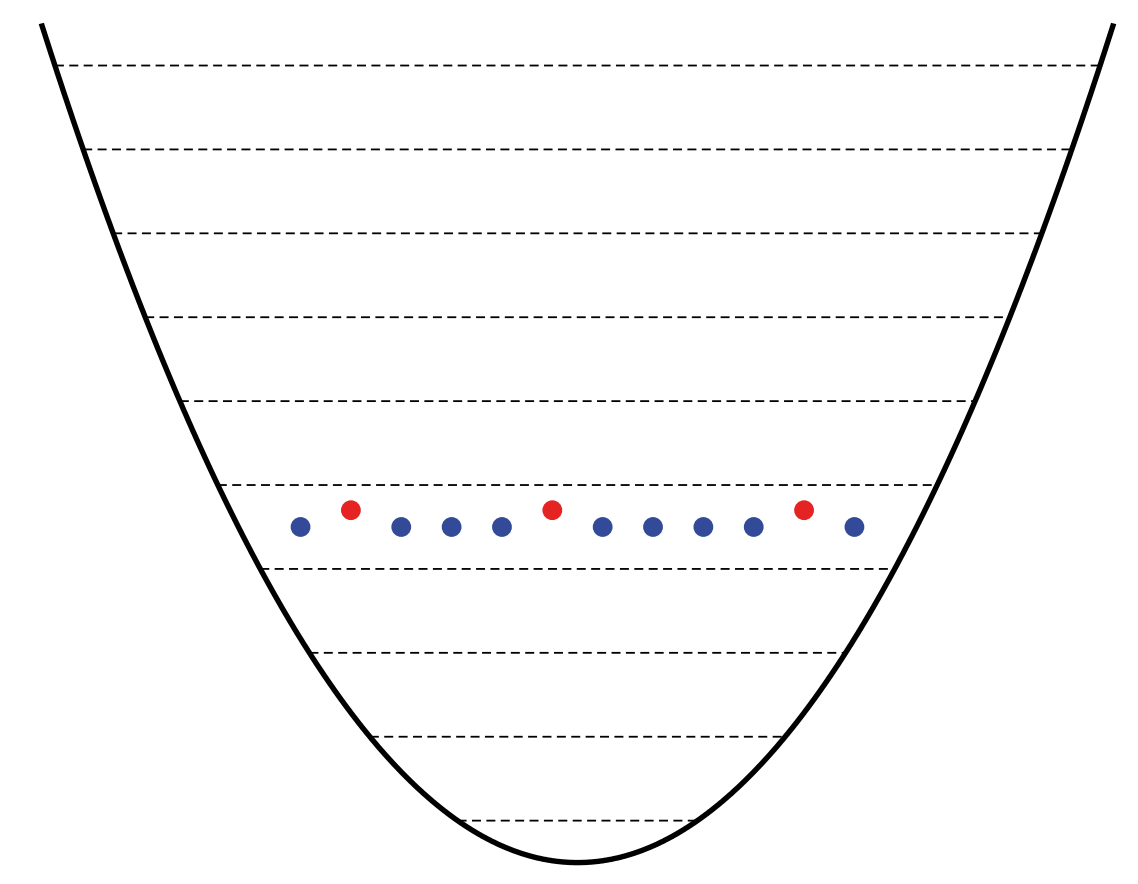

$\rightarrow$ not all atoms share the same wavefunction: finite "depletion" at zero temperature

$$
\delta N \equiv N_{\text {noncondensed }} \equiv N-N_{0} \sim\left(N a_{s} / a_{\text {osc }}\right)^{6 / 5} \text { for small } a_{s}
$$




\section{BEC with atom-atom interaction}

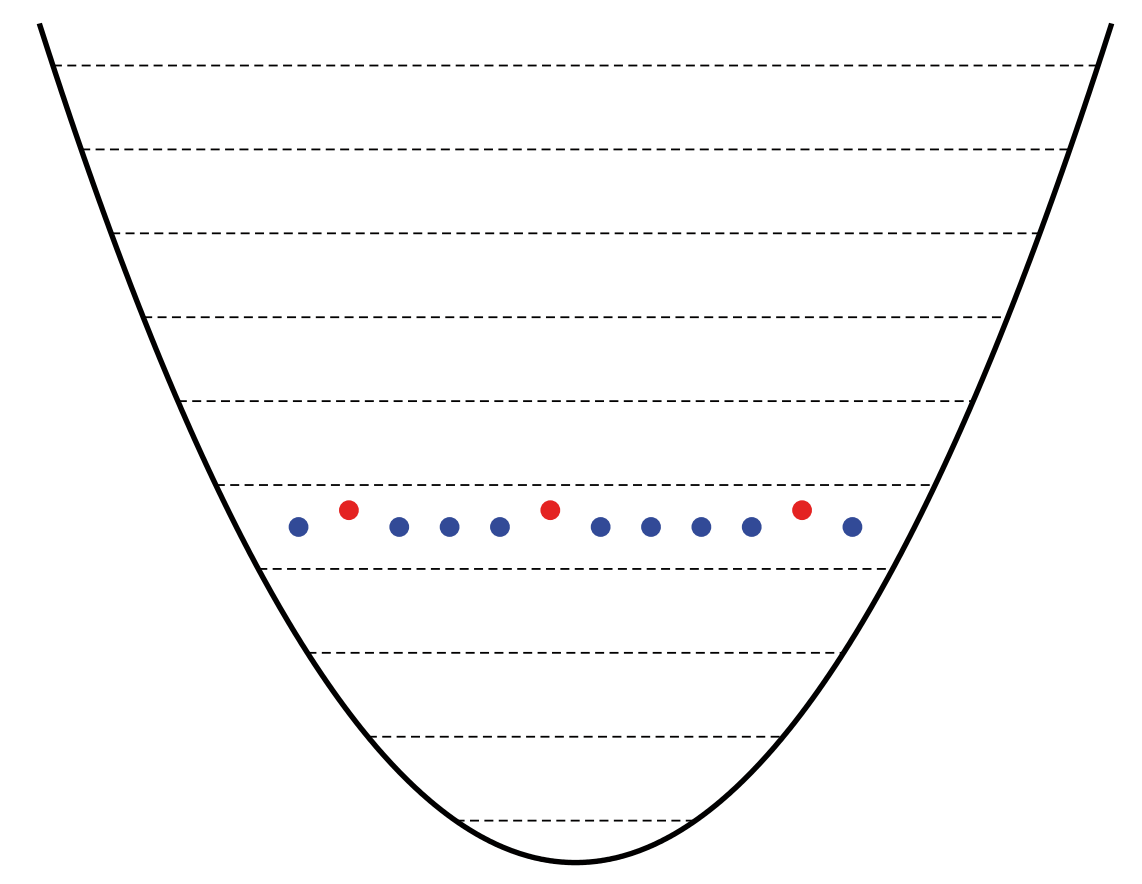

Mean-field limit: $N \rightarrow \infty, a_{s} \rightarrow 0$, such that $N a_{s} \sim$ const

$\longrightarrow \delta N \sim\left(N a_{s}\right)^{6 / 5} \sim$ const $\sim \mathcal{O}\left(N^{0}\right)$

$\longrightarrow$ Gross-Pitaevskii description becomes perfect in that limit

E. H. Lieb and R. Seiringer and J. Yngvason, PRA 61, 043602 (2000) 


\section{BEC with atom-atom interaction}

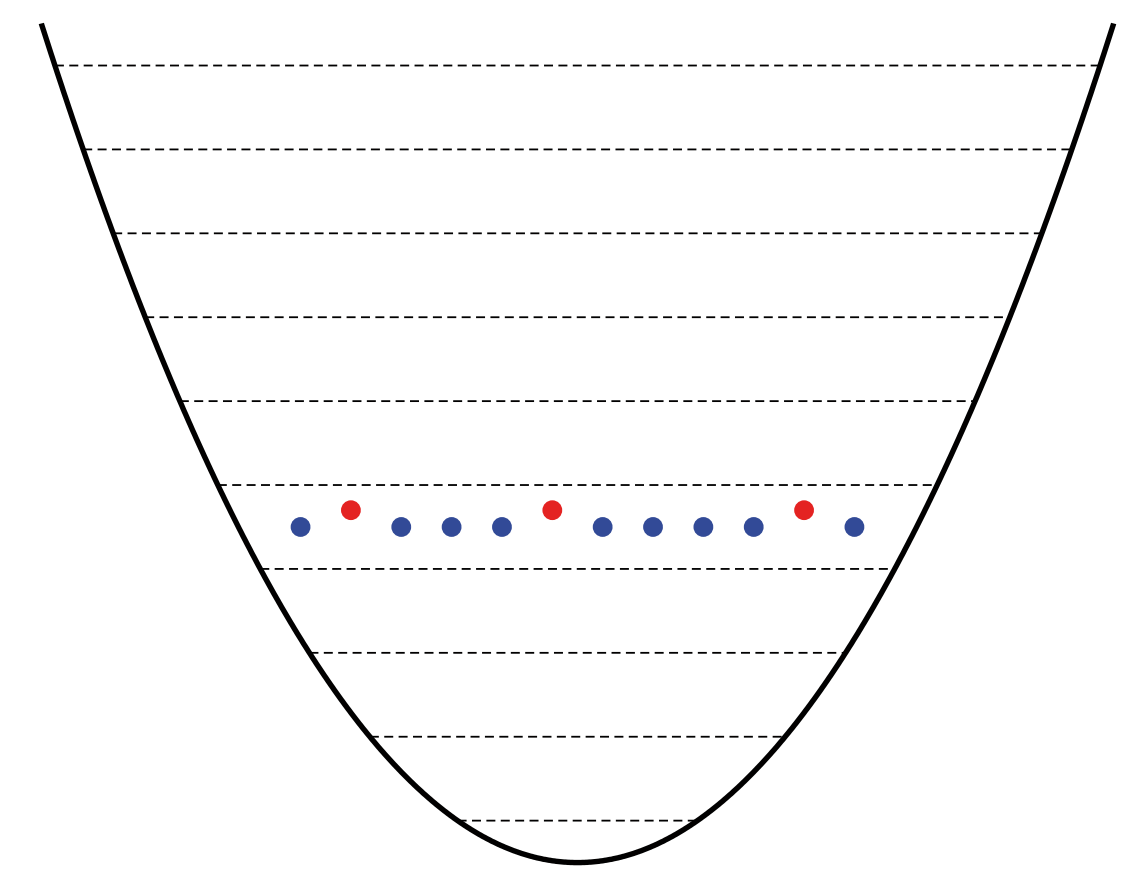

$\longrightarrow$ spontaneous breaking of $U(1)$ gauge symmetry for $N \rightarrow \infty$

$\longrightarrow$ atomic field operator $\hat{\psi}(\mathbf{r})$ can be replaced by a c-number $\psi(\mathbf{r}) \equiv\langle\hat{\psi}(\mathbf{r})\rangle$ for the condensate

E. H. Lieb and R. Seiringer and J. Yngvason, Rep. Math. Phys. 59, 389 (2007) 


\section{BEC with interaction at finite temperature}

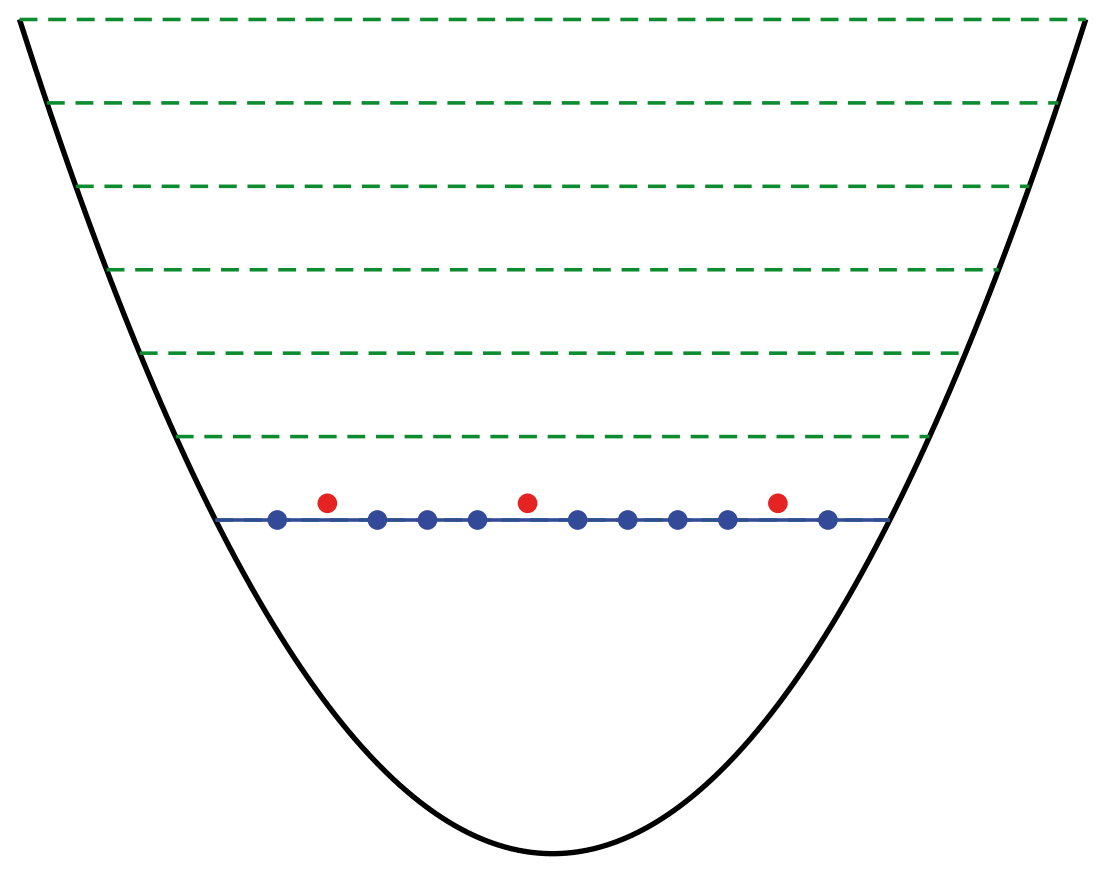

$\longrightarrow$ thermal population of Bogoliubov quasiparticle excitations

$$
\hat{H}_{\mathrm{QP}}=\sum_{j=1}^{\infty} \hbar \omega_{j} \hat{\alpha}_{j}^{\dagger} \hat{\alpha}_{j}
$$

(quantization of collective modes within the condensate) 


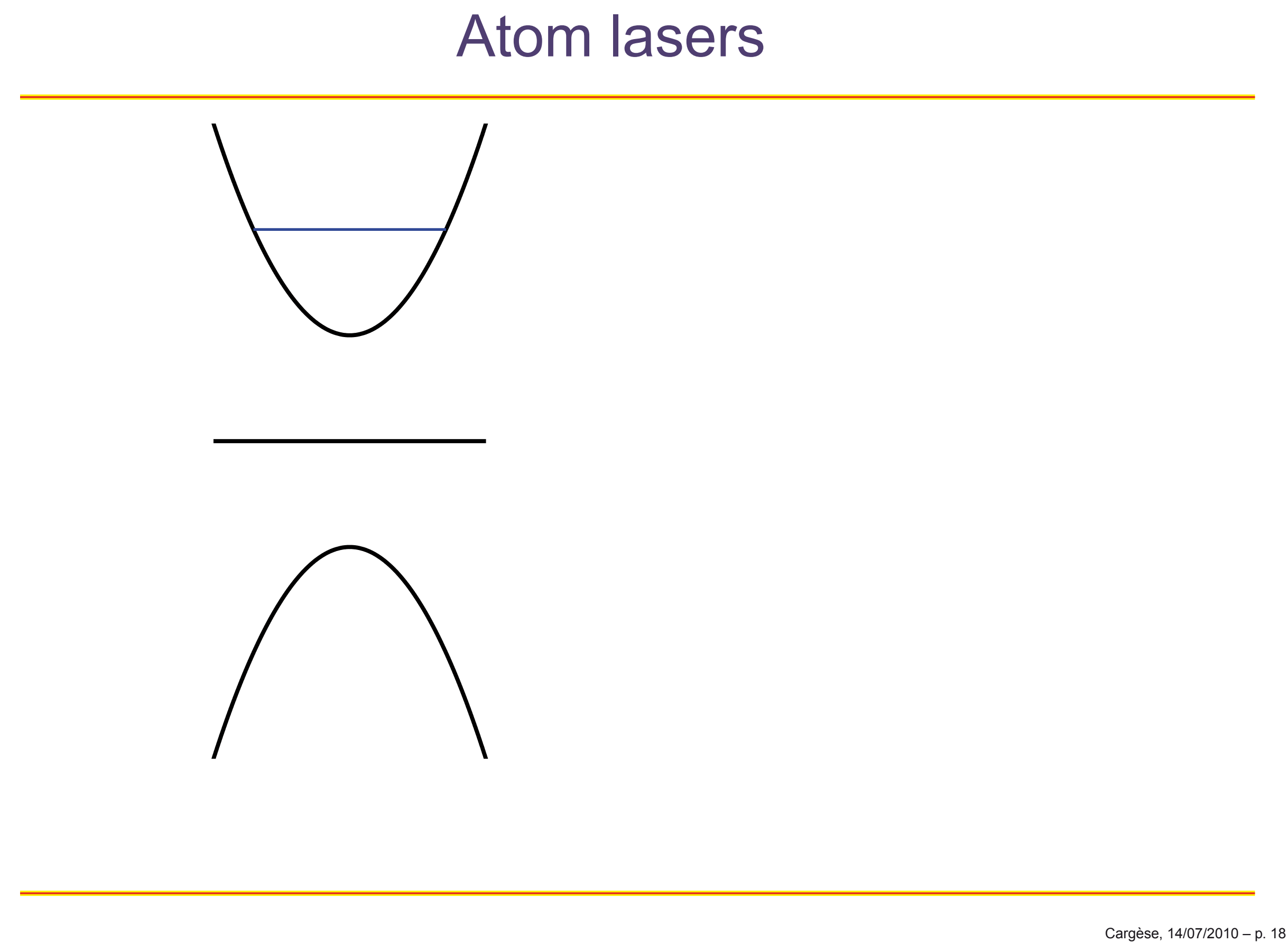




\section{Atom lasers}

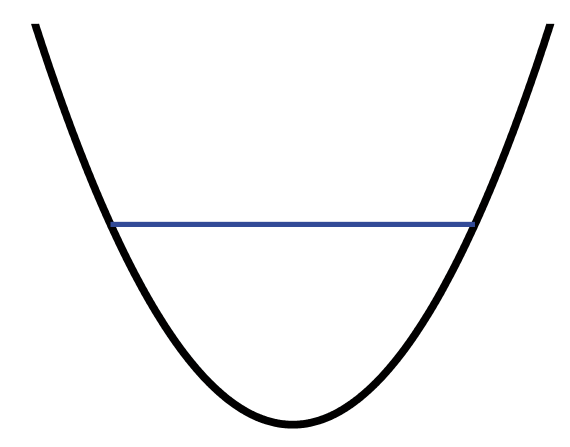

Level scheme of ${ }^{87} \mathrm{Rb}$ :
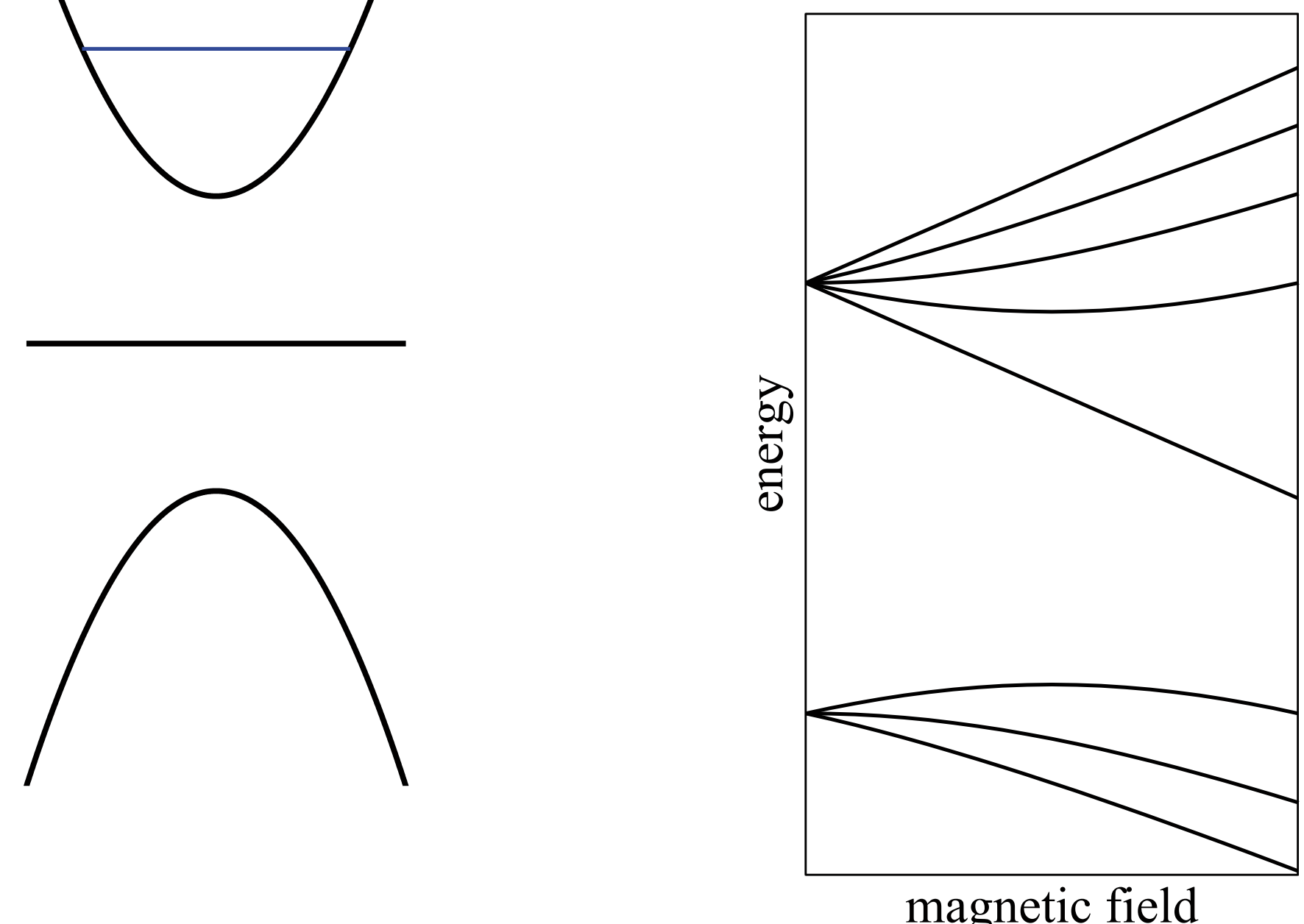


\section{Atom lasers}

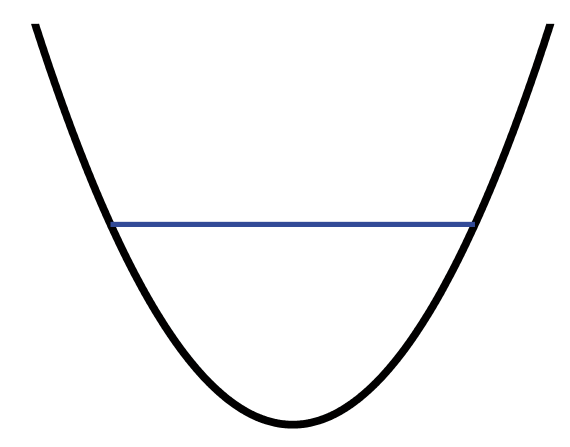

Level scheme of ${ }^{87} \mathrm{Rb}$ :
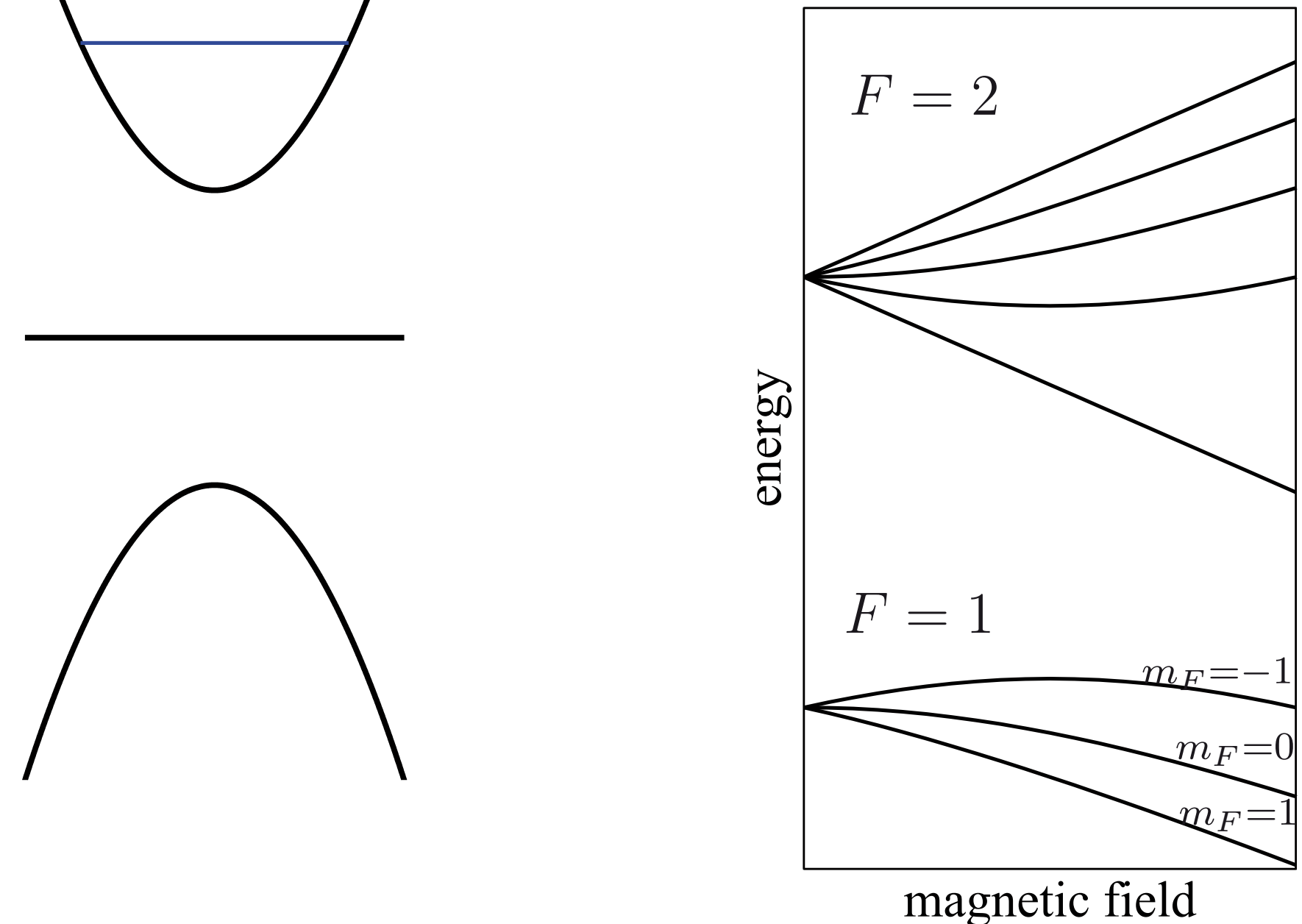


\section{Atom lasers}

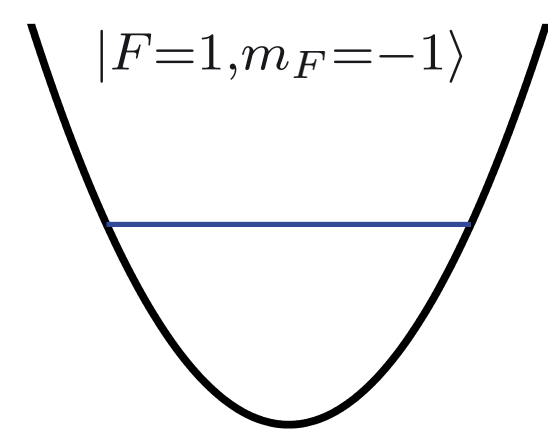

Level scheme of ${ }^{87} \mathrm{Rb}$ :
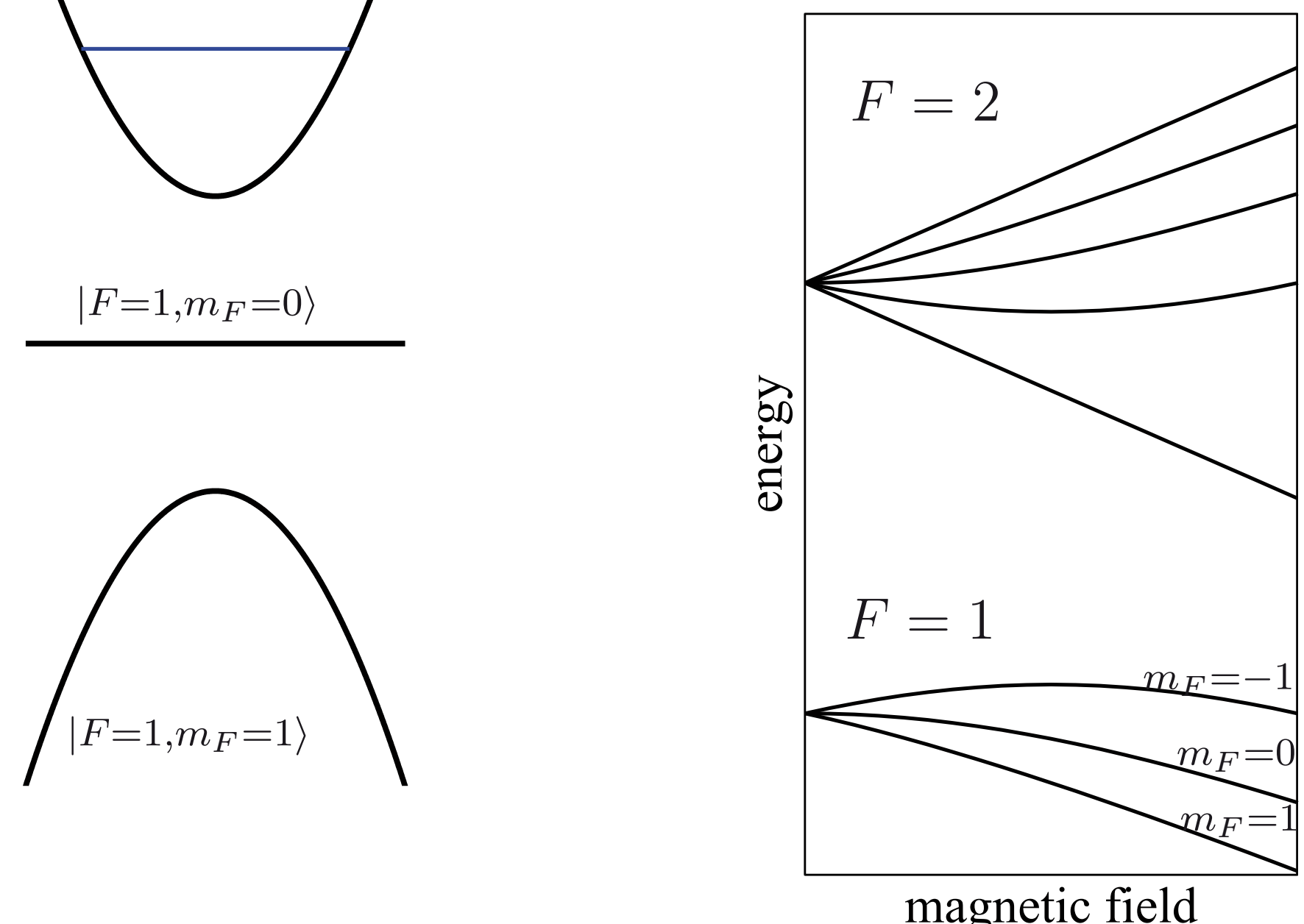


\section{Atom lasers}

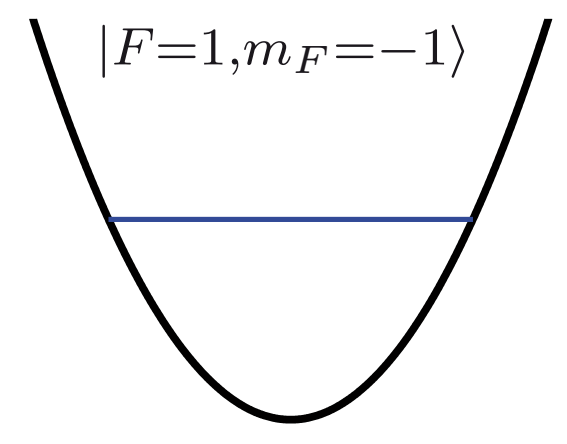

Apply a radiofrequency field
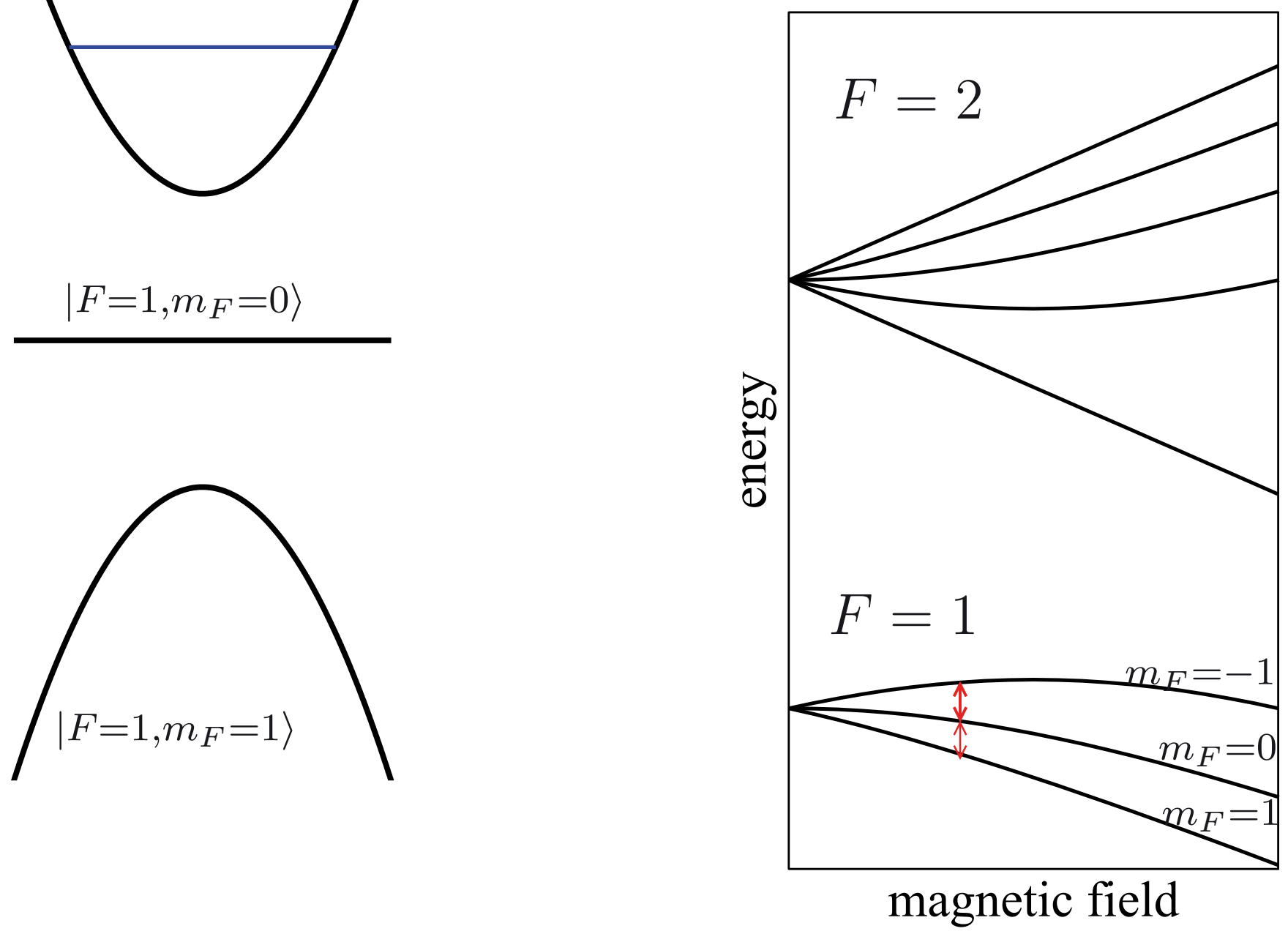


\section{Atom lasers}

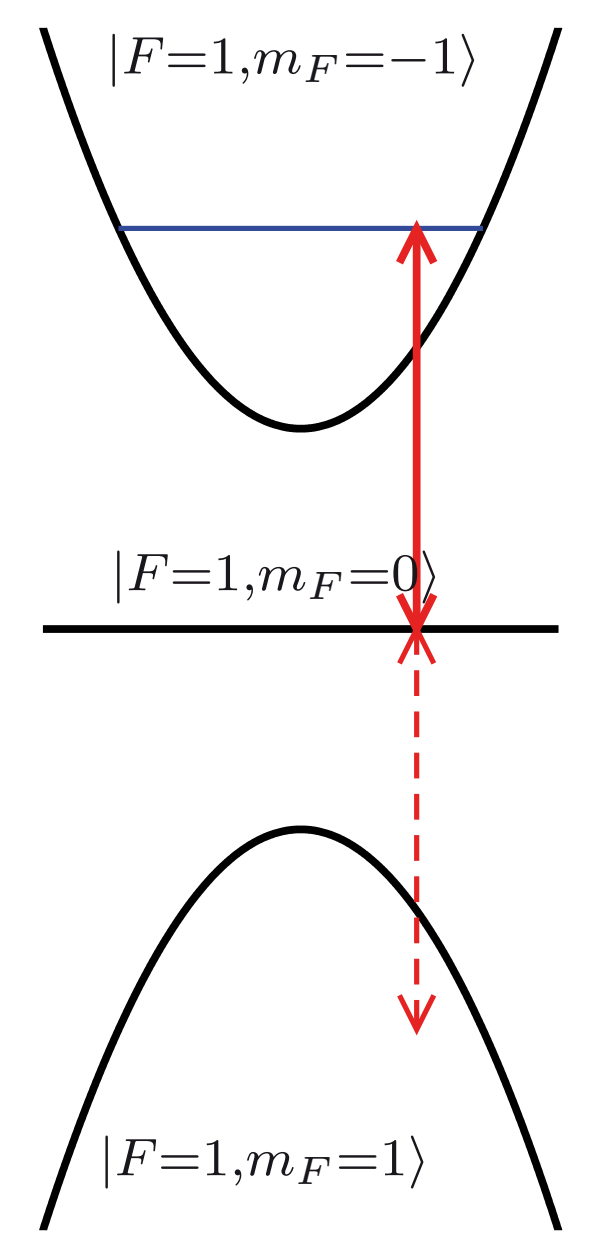

Apply a radiofrequency field

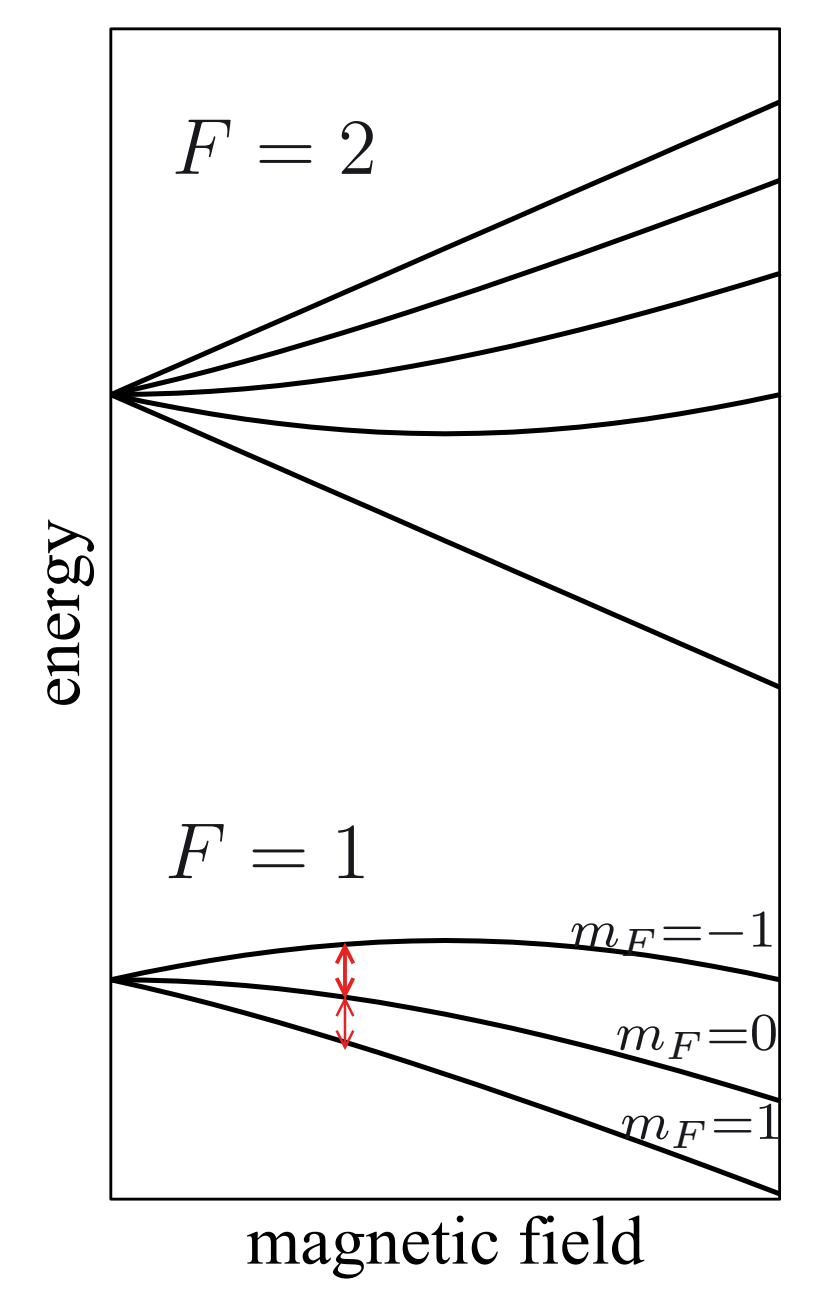




\section{Atom lasers with gravity}
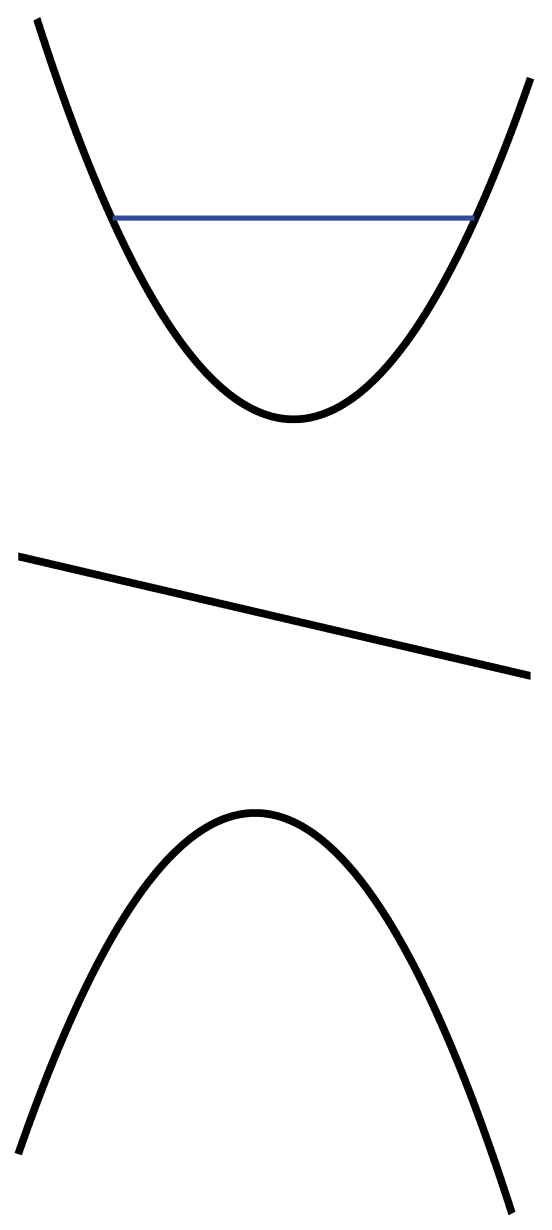


\section{Atom lasers with gravity}
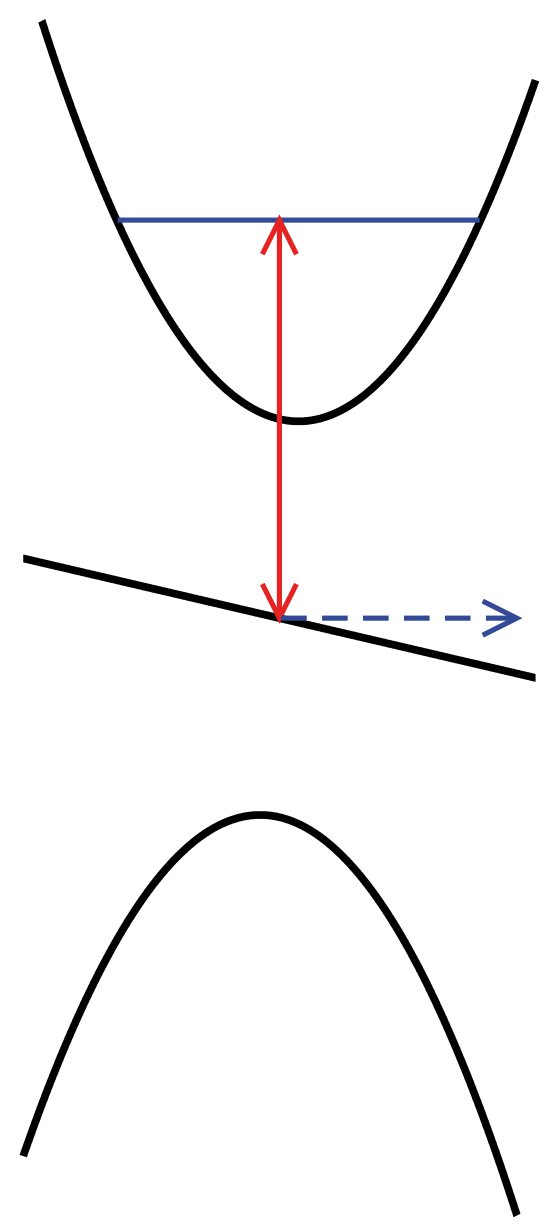


\section{Atom lasers with gravity}

\section{Atom Laser with a cw Output Coupler}

Immanuel Bloch, Theodor W. Hänsch, and Tilman Esslinger Sektion Physik, Ludwig-Maximilians-Universität, Schellingstrasse 4/III, D-80799 Munich, Germany and Max-Planck-Institut für Quantenoptik, D-85748 Garching, Germany (Received 3 December 1998)

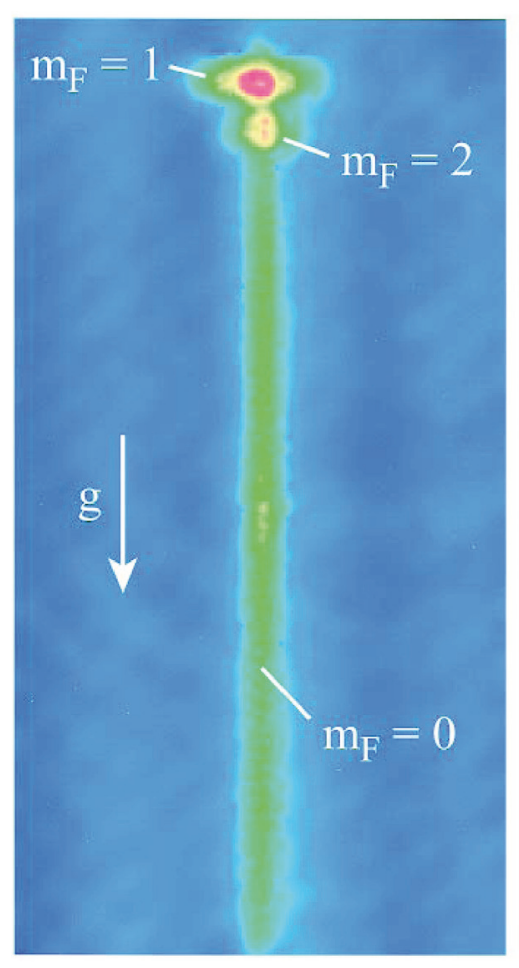




\section{Guided atom laser}

Idea: add a horizontal optical waveguide (focused laser beam)

magnetic trap

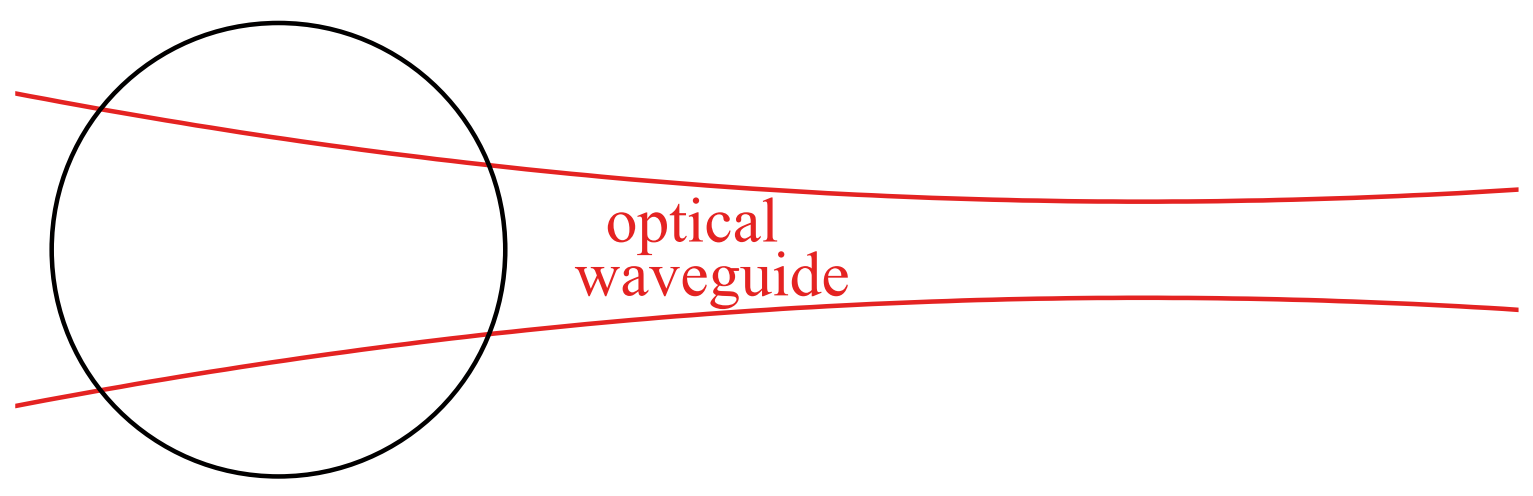




\section{Guided atom laser}

Idea: add a horizontal optical waveguide (focused laser beam)

magnetic trap
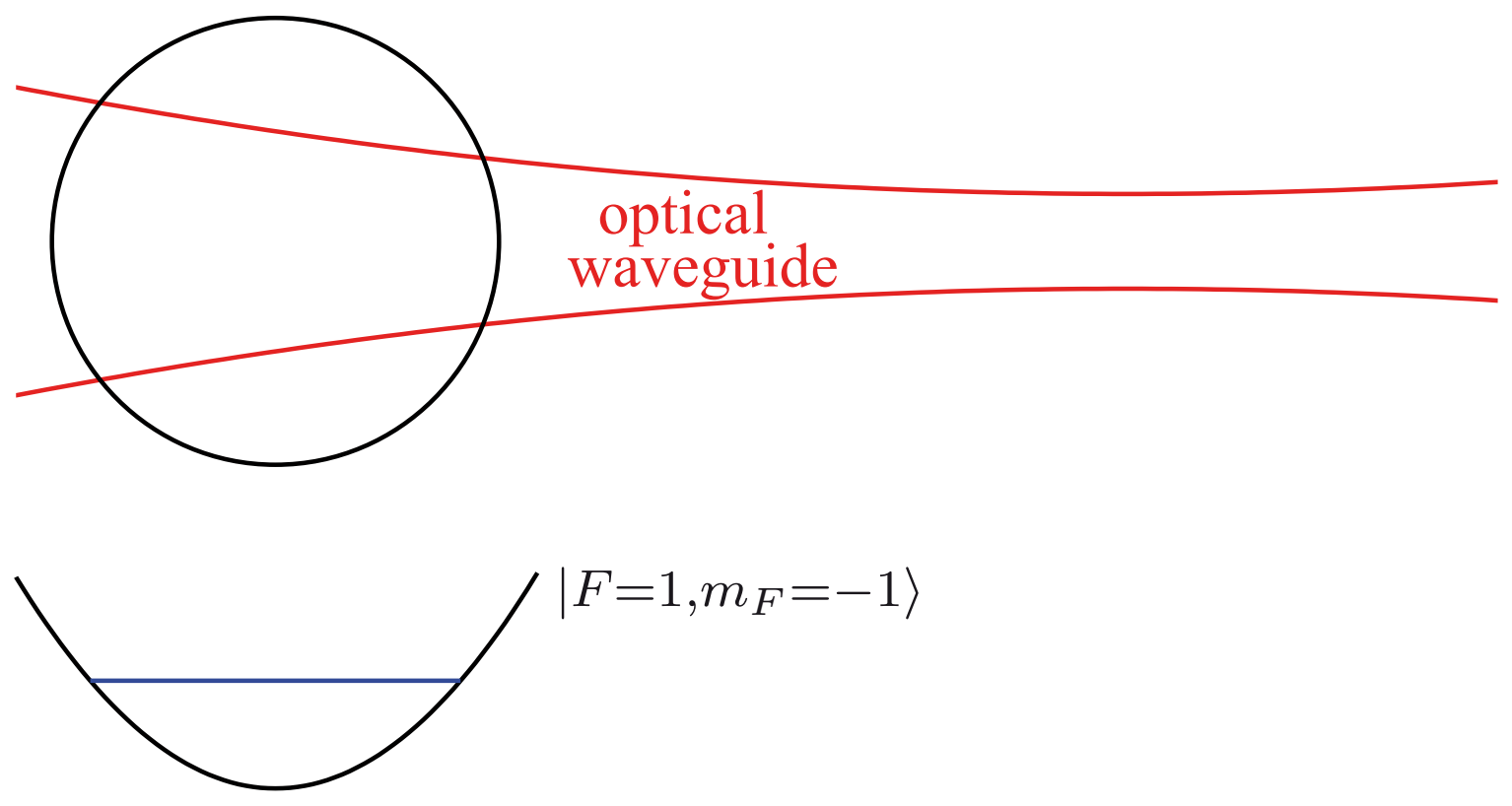

$$
\left|F=1, m_{F}=0\right\rangle
$$




\section{Guided atom laser}

Idea: add a horizontal optical waveguide (focused laser beam)

magnetic trap
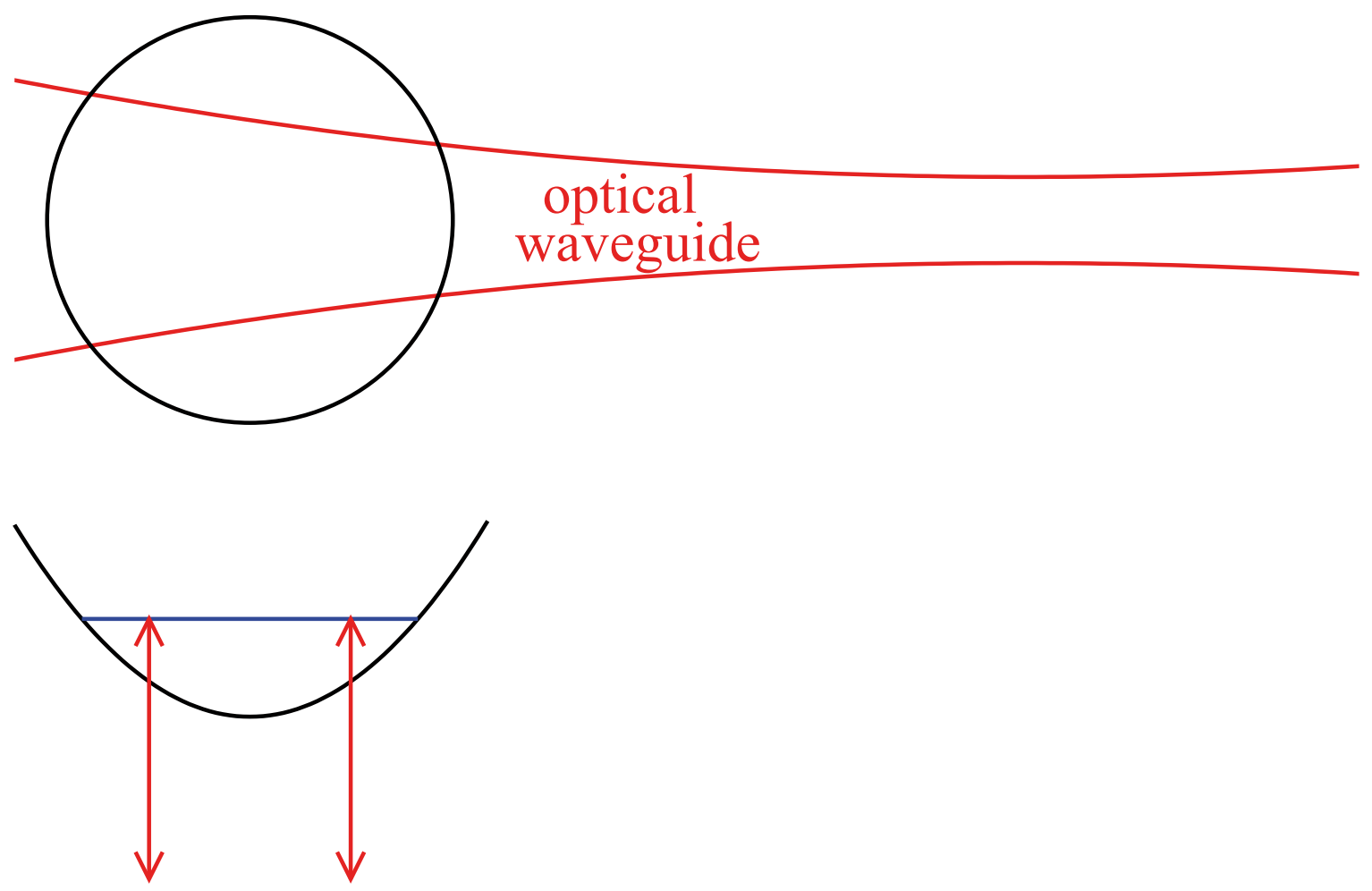


\section{Guided atom laser}

Idea: add a horizontal optical waveguide (focused laser beam)

magnetic trap
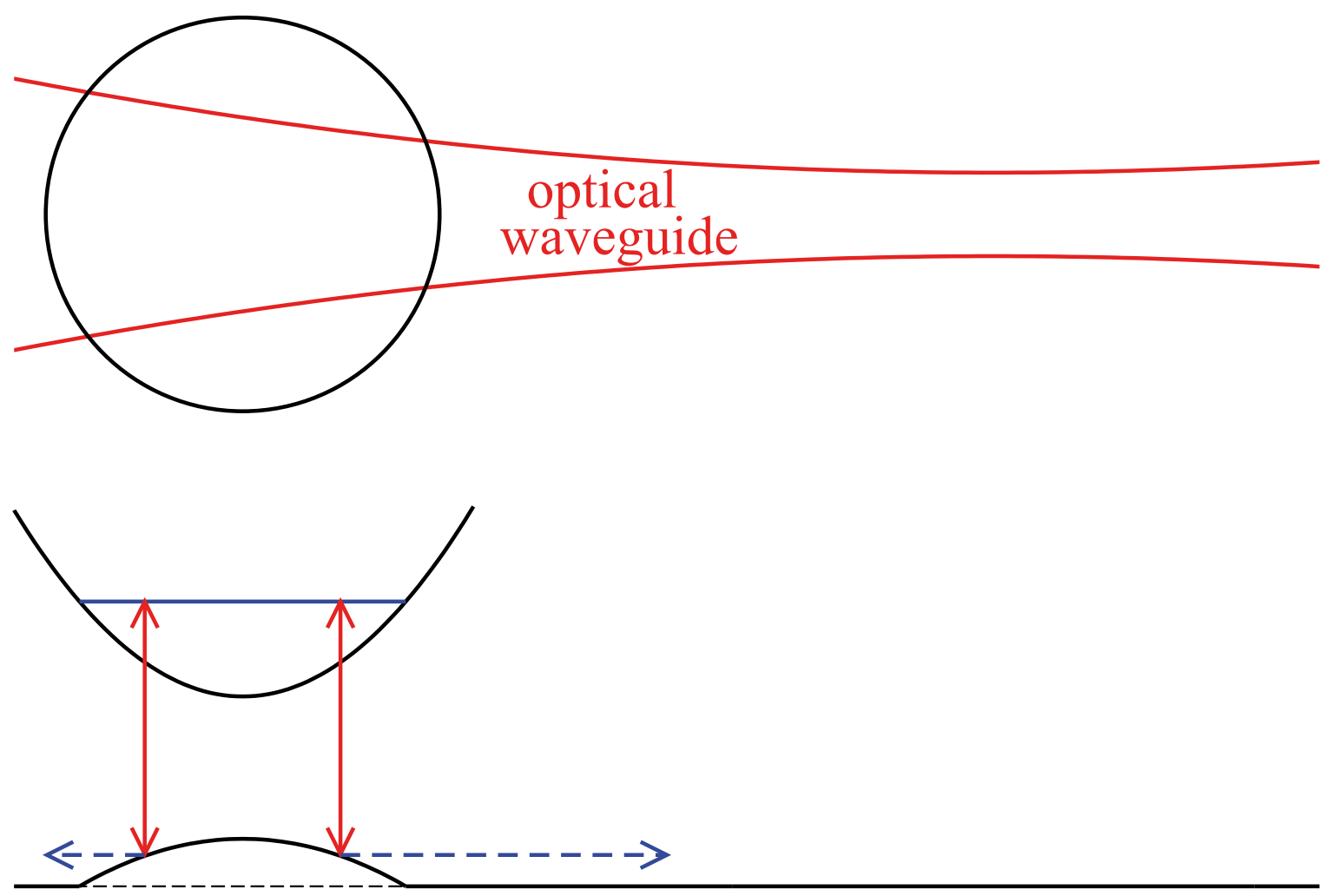

repulsive interaction between $m_{F}=-1$ and $m_{F}=0$ atoms 


\section{Guided atom laser}

Idea: add a horizontal optical waveguide (focused laser beam)

magnetic trap
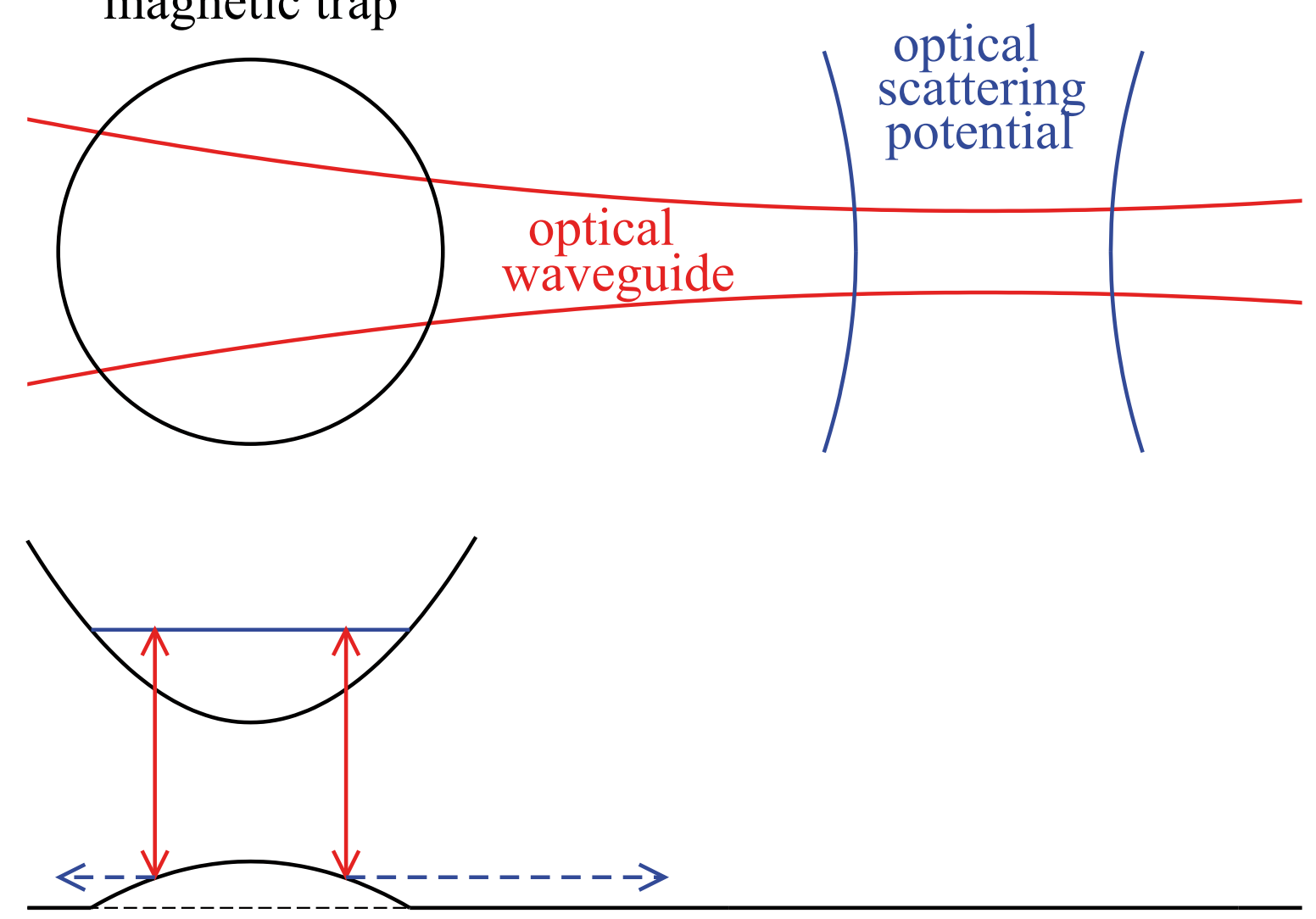


\section{Guided atom laser}

Idea: add a horizontal optical waveguide (focused laser beam)

magnetic trap
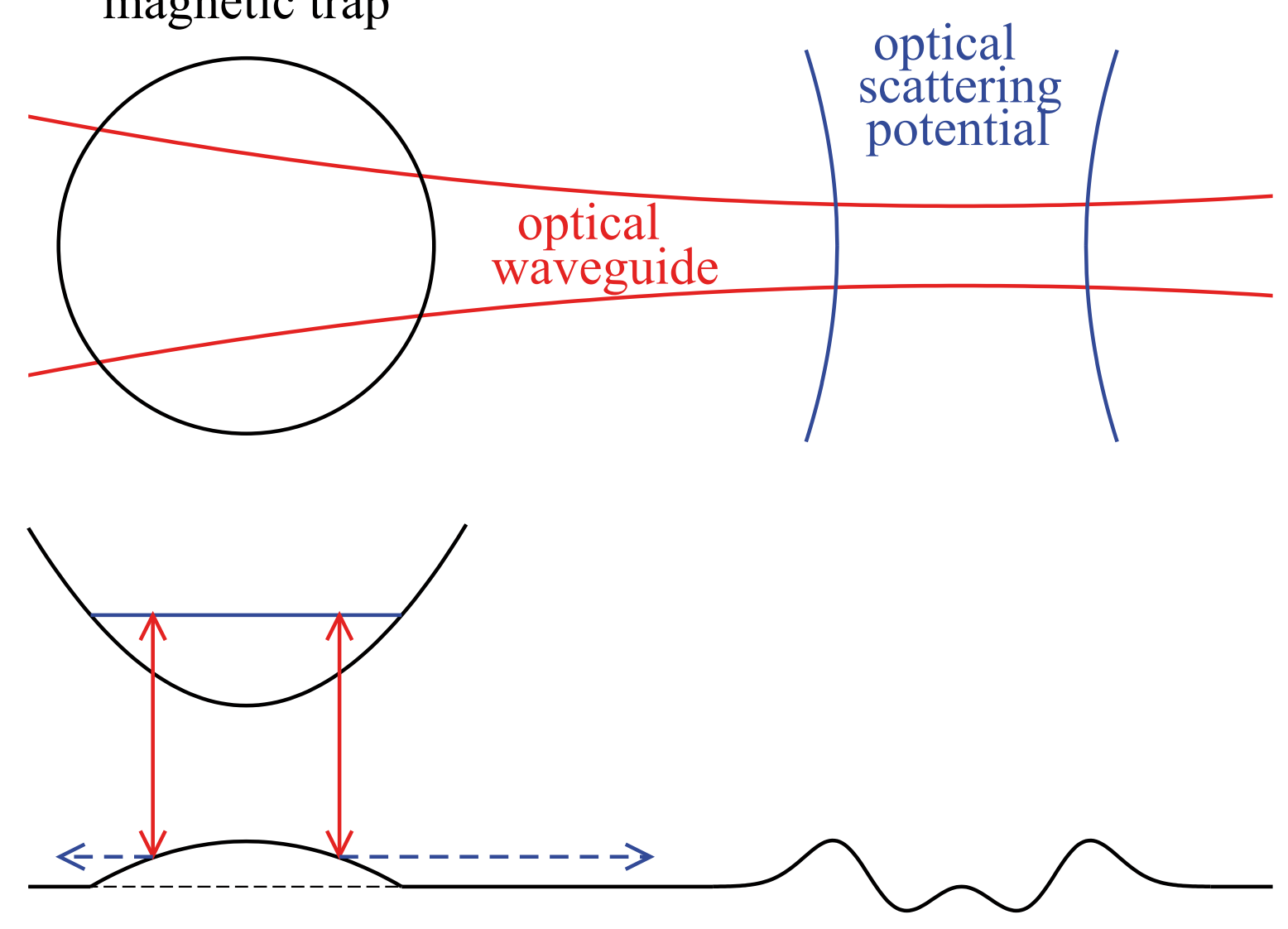


\section{Guided atom laser}

Idea: add a horizontal optical waveguide (focused laser beam)
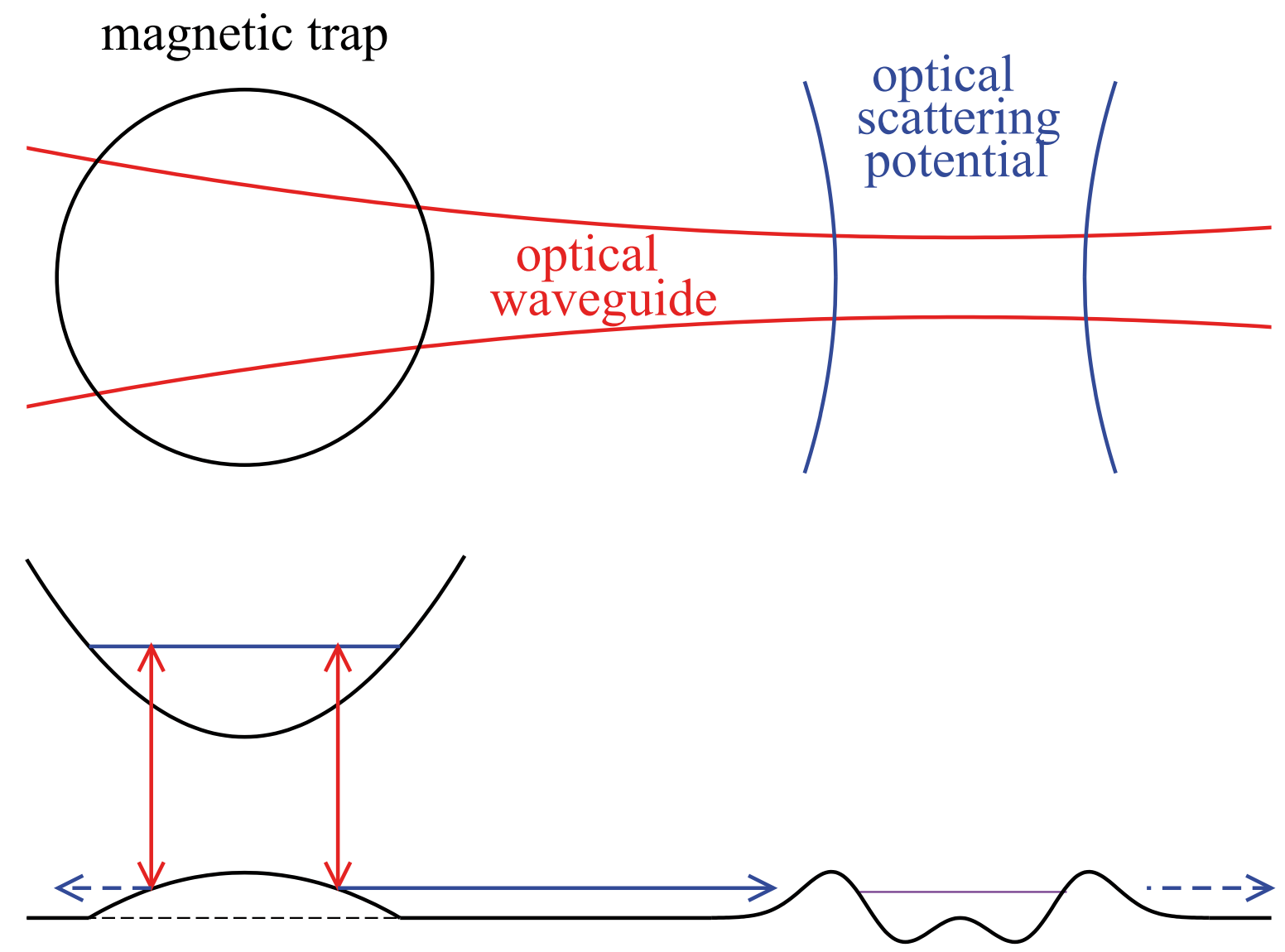

$\longrightarrow$ study quasistationary scattering at well-defined energy 


\section{Guided atom laser}

Idea: add a horizontal optical waveguide (focused laser beam)
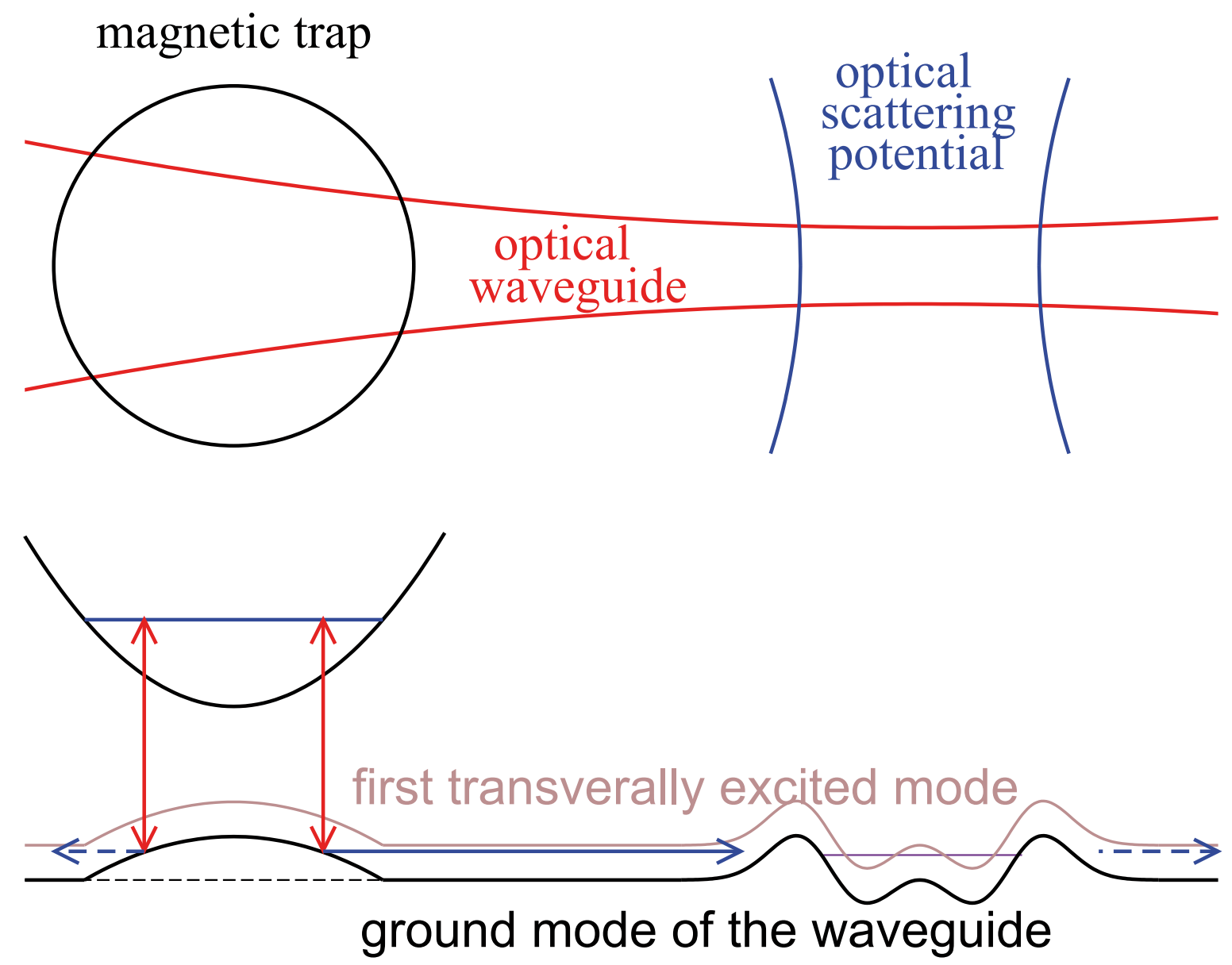


\section{Guided atom laser}

Idea: add a horizontal optical waveguide (focused laser beam)

a)

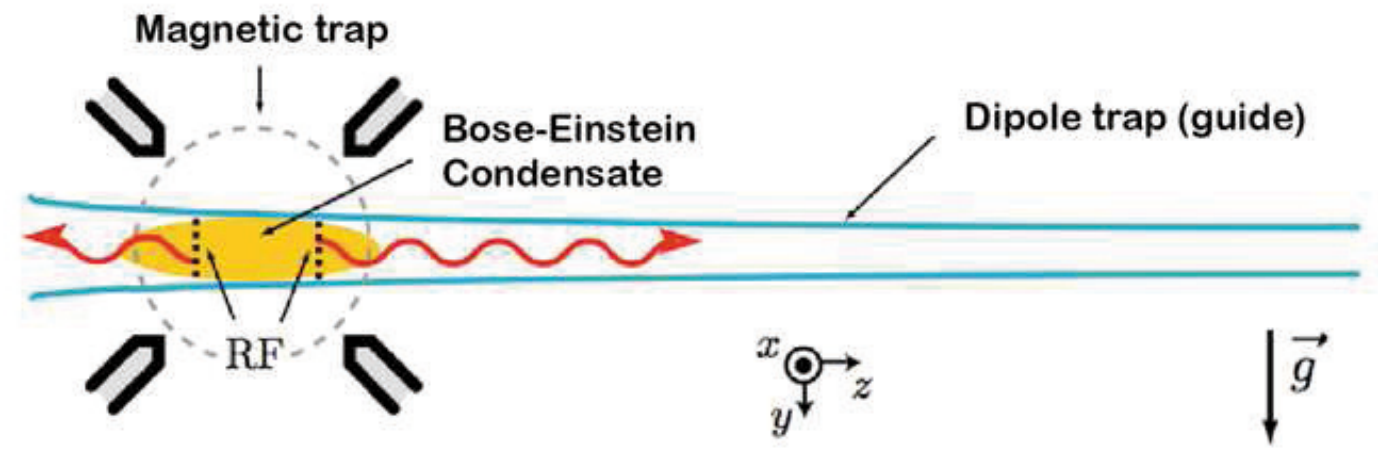

b)

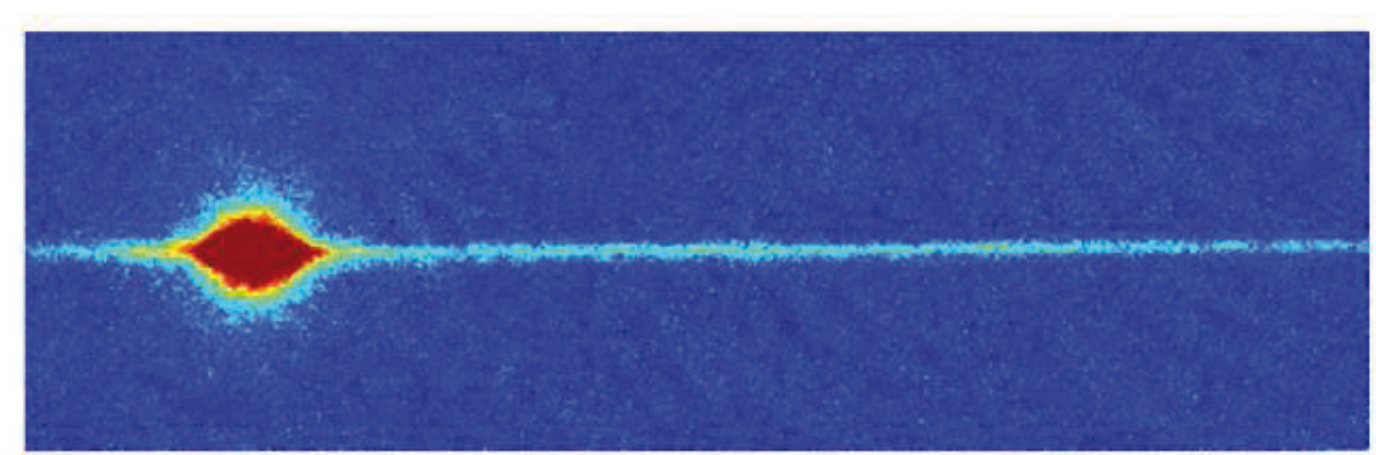

$\longrightarrow$ experimental realization: W. Guerin et al., PRL 97, 200402 (2006) 


\section{Guided atom laser}

Idea: add a horizontal optical waveguide (focused laser beam)

a)

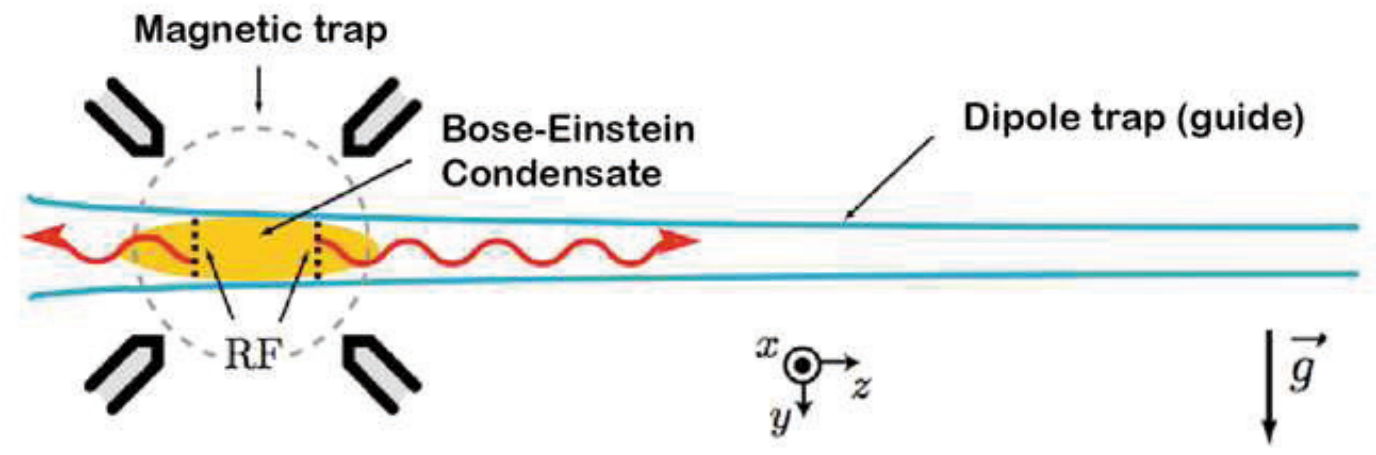

b)

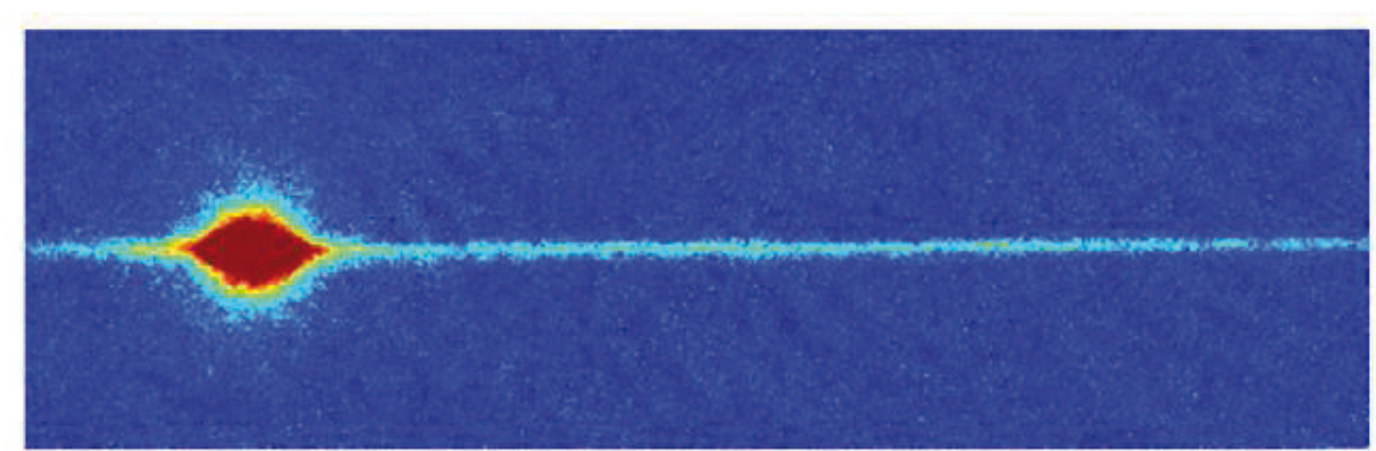

$\longrightarrow$ experimental realization: W. Guerin et al., PRL 97, 200402 (2006)

$\longrightarrow$ all-optical realization of a guided atom laser:

A. Couvert et al., EPL 83, 50001 (2008) 


\section{Transport theory of Bose-Einstein condensates}

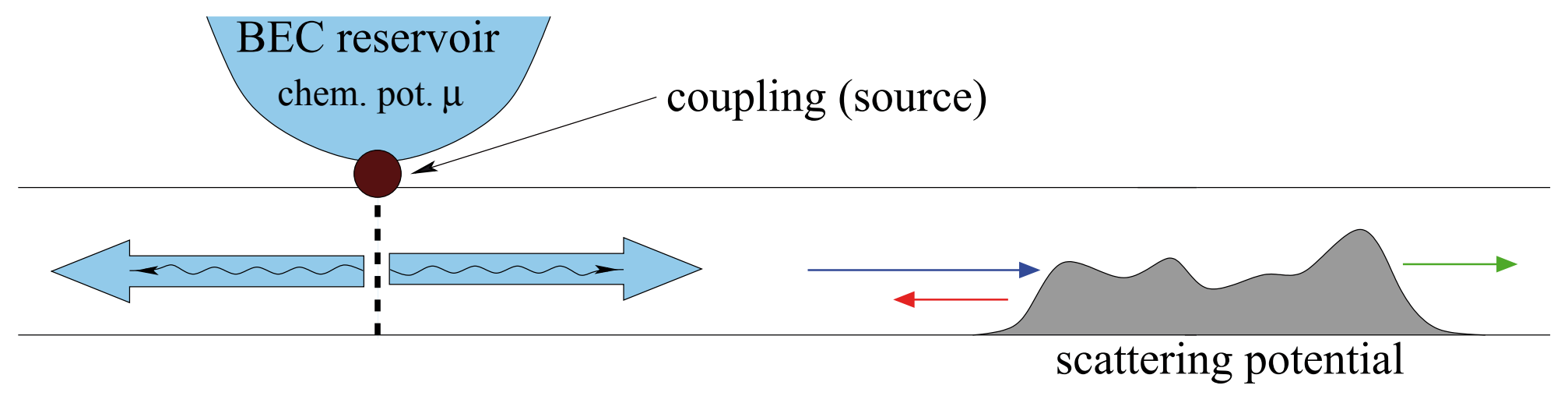

Field equation for atoms in the waveguide:

$$
\begin{aligned}
i \hbar \frac{\partial}{\partial t} \hat{\Psi}(\mathbf{r}, t)= & \left(-\frac{\hbar^{2}}{2 m} \Delta+V_{\text {guide }}(\mathbf{r})+U \hat{\Psi}^{\dagger}(\mathbf{r}, t) \hat{\Psi}(\mathbf{r}, t)\right) \hat{\Psi}(\mathbf{r}, t) \\
& +\tilde{U} \hat{\Psi}_{\text {res }}^{\dagger}(\mathbf{r}, t) \hat{\Psi}_{\mathrm{res}}(\mathbf{r}, t) \hat{\Psi}(\mathbf{r}, t)+K(t) \hat{\Psi}_{\mathrm{res}}(\mathbf{r}, t)
\end{aligned}
$$




\section{Transport theory of Bose-Einstein condensates}

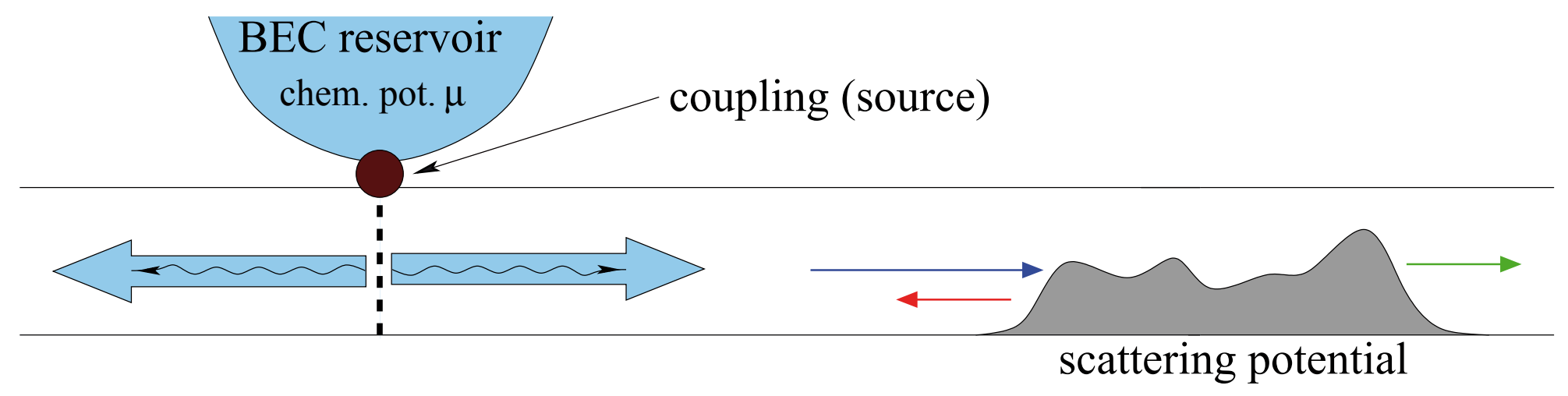

Field equation for atoms in the waveguide:

$$
\begin{aligned}
i \hbar \frac{\partial}{\partial t} \hat{\Psi}(\mathbf{r}, t)= & \left(-\frac{\hbar^{2}}{2 m} \Delta+V_{\text {guide }}(\mathbf{r})+U \hat{\Psi}^{\dagger}(\mathbf{r}, t) \hat{\Psi}(\mathbf{r}, t)\right) \hat{\Psi}(\mathbf{r}, t) \\
& +\tilde{U} \hat{\Psi}_{\mathrm{res}}^{\dagger}(\mathbf{r}, t) \hat{\Psi}_{\mathrm{res}}(\mathbf{r}, t) \hat{\Psi}(\mathbf{r}, t)+K(t) \hat{\Psi}_{\mathrm{res}}(\mathbf{r}, t)
\end{aligned}
$$

Perfect condensation in the reservoir: $\hat{\Psi}_{\text {res }}(\mathbf{r}, t)=\Psi_{\text {res }}(\mathbf{r}) e^{-i \mu t / \hbar}$ (mean-field limit: $N \rightarrow \infty$ ) 


\section{Transport theory of Bose-Einstein condensates}

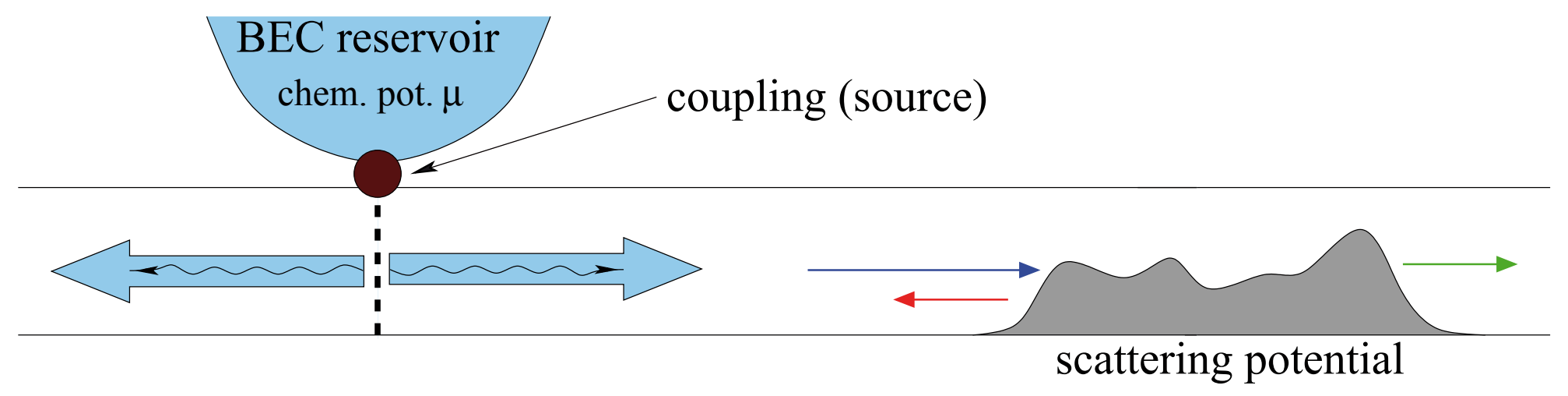

Field equation for atoms in the waveguide:

$$
\begin{aligned}
i \hbar \frac{\partial}{\partial t} \hat{\Psi}(\mathbf{r}, t)= & \left(-\frac{\hbar^{2}}{2 m} \Delta+V_{\text {guide }}(\mathbf{r})+U \hat{\Psi}^{\dagger}(\mathbf{r}, t) \hat{\Psi}(\mathbf{r}, t)\right) \hat{\Psi}(\mathbf{r}, t) \\
& +\tilde{U}\left|\Psi_{\text {res }}(\mathbf{r})\right|^{2} \hat{\Psi}(\mathbf{r}, t)+K(t) \Psi_{\text {res }}(\mathbf{r}) \exp (-i \mu t / \hbar)
\end{aligned}
$$

$\longrightarrow$ inhomogeneous field equation with a coherent source 


\section{Transport theory of Bose-Einstein condensates}

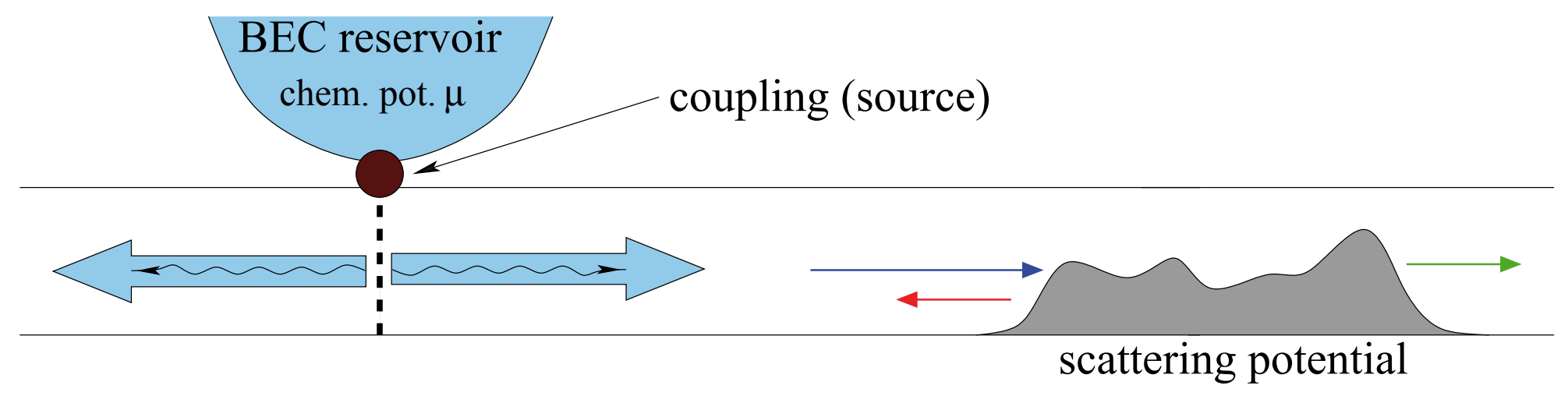

Field equation for atoms in the waveguide:

$$
\begin{aligned}
i \hbar \frac{\partial}{\partial t} \hat{\Psi}(\mathbf{r}, t)= & \left(-\frac{\hbar^{2}}{2 m} \Delta+V_{\text {guide }}(\mathbf{r})+U \hat{\Psi}^{\dagger}(\mathbf{r}, t) \hat{\Psi}(\mathbf{r}, t)\right) \hat{\Psi}(\mathbf{r}, t) \\
& +\tilde{U}\left|\Psi_{\text {res }}(\mathbf{r})\right|^{2} \hat{\Psi}(\mathbf{r}, t)+K(t) \Psi_{\text {res }}(\mathbf{r}) \exp (-i \mu t / \hbar)
\end{aligned}
$$

Restriction to the transverse ground mode of the waveguide:

$$
\hat{\Psi}(\mathbf{r}, t)=\chi_{0}\left(\mathbf{r}_{\perp}\right) \exp \left(-i \omega_{\perp} t\right) \hat{\psi}(x, t)
$$




\section{Transport theory of Bose-Einstein condensates}

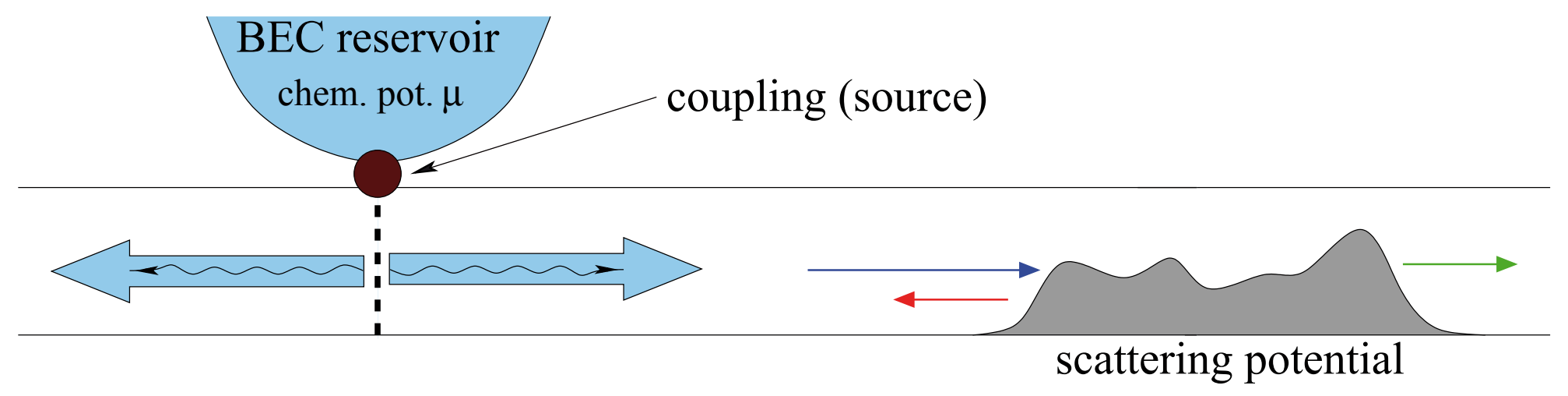

Field equation for atoms in the waveguide:

$$
\begin{aligned}
i \hbar \frac{\partial}{\partial t} \hat{\psi}(x, t)= & \left(-\frac{\hbar^{2}}{2 m} \frac{\partial^{2}}{\partial x^{2}}+V(x)+g \hat{\psi}^{\dagger}(x, t) \hat{\psi}(x, t)\right) \hat{\psi}(x, t) \\
& +\underbrace{\int d^{2} r_{\perp} \chi_{0}^{*}\left(\mathbf{r}_{\perp}\right) K(t) \Psi_{\text {res }}(\mathbf{r})}_{S(x, t): \text { coherent source }} \exp (-i \mu t / \hbar)
\end{aligned}
$$

with $g=2 \hbar \omega_{\perp} a_{s}$ : effective 1D interaction strength 


\section{Transport theory of Bose-Einstein condensates}

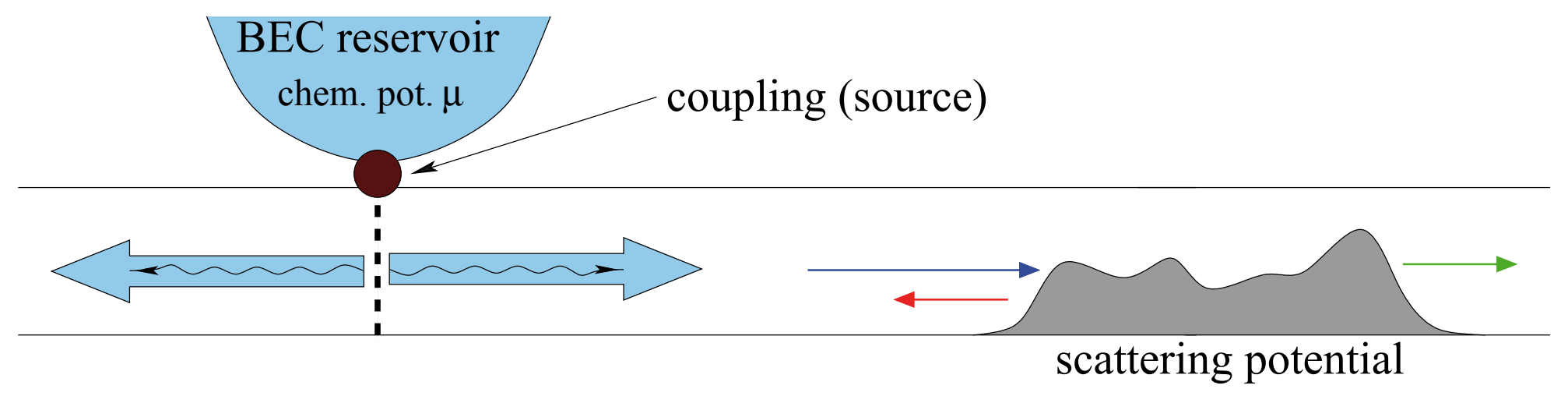

Field equation for atoms in the waveguide:

$$
\begin{aligned}
i \hbar \frac{\partial}{\partial t} \hat{\psi}(x, t)= & \left(-\frac{\hbar^{2}}{2 m} \frac{\partial^{2}}{\partial x^{2}}+V(x)+g \hat{\psi}^{\dagger}(x, t) \hat{\psi}(x, t)\right) \hat{\psi}(x, t) \\
& +\underbrace{\int d^{2} r_{\perp} \chi_{0}^{*}\left(\mathbf{r}_{\perp}\right) K(t) \Psi_{\text {res }}(\mathbf{r})}_{S(x, t): \text { coherent source }} \exp (-i \mu t / \hbar)
\end{aligned}
$$

with $g=\frac{2 \hbar \omega_{\perp} a_{s}}{1-1.46 a_{s} / a_{\perp}^{\text {(osc) }}}$ M. Olshanii, PRL 81, 938 (1998) 


\section{Transport theory of Bose-Einstein condensates}

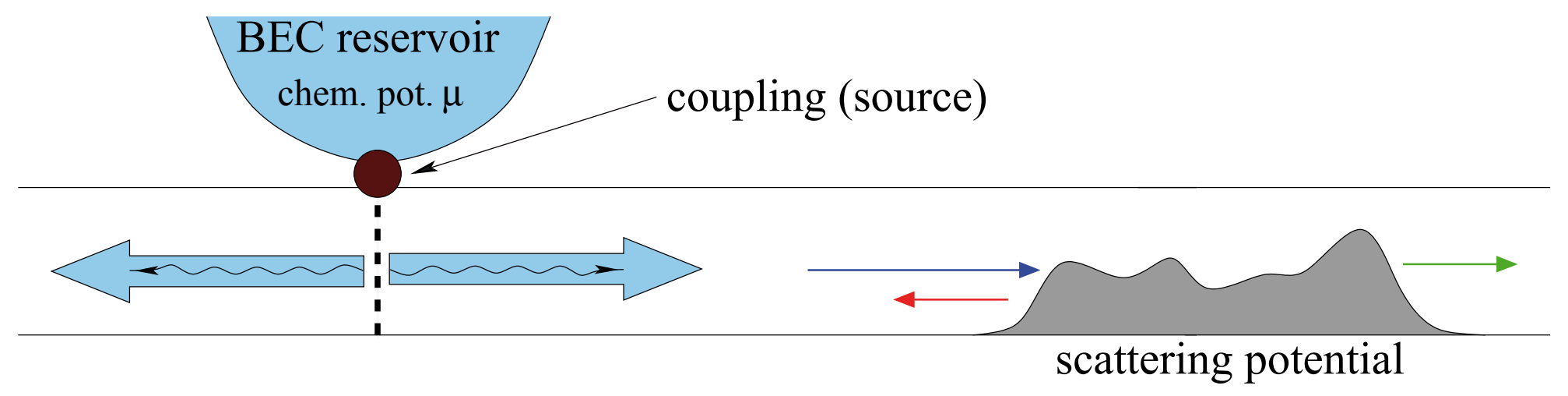

Field equation for atoms in the waveguide:

$$
\begin{aligned}
i \hbar \frac{\partial}{\partial t} \hat{\psi}(x, t)= & \left(-\frac{\hbar^{2}}{2 m} \frac{\partial^{2}}{\partial x^{2}}+V(x)+g \hat{\psi}^{\dagger}(x, t) \hat{\psi}(x, t)\right) \hat{\psi}(x, t) \\
& +S(x, t) \exp (-i \mu t / \hbar)
\end{aligned}
$$

Mean-field approximation:

$$
\hat{\psi}(x, t)=\langle\hat{\psi}(x, t)\rangle+\delta \hat{\psi}(x, t) \equiv \psi(x, t)
$$




\section{Transport theory of Bose-Einstein condensates}

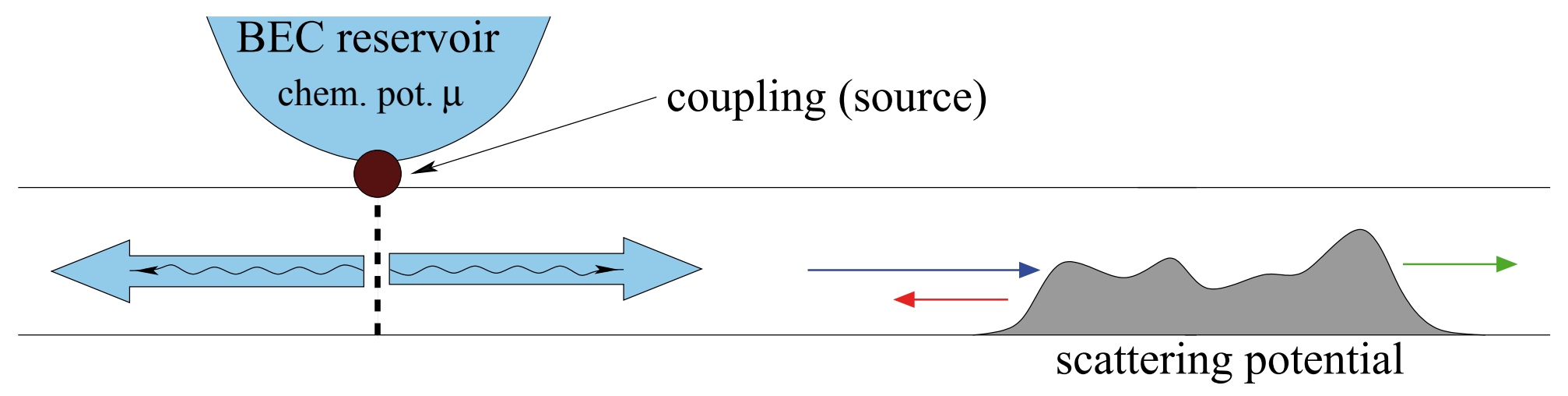

Field equation for atoms in the waveguide:

$$
\begin{aligned}
i \hbar \frac{\partial}{\partial t} \psi(x, t)= & \left(-\frac{\hbar^{2}}{2 m} \frac{\partial^{2}}{\partial x^{2}}+V(x)+g|\psi(x, t)|^{2}\right) \psi(x, t) \\
& +S(x, t) \exp (-i \mu t / \hbar)
\end{aligned}
$$

$\longrightarrow$ inhomogeneous 1D Gross-Pitaevskii equation 


\section{Scattering through double barrier potentials}

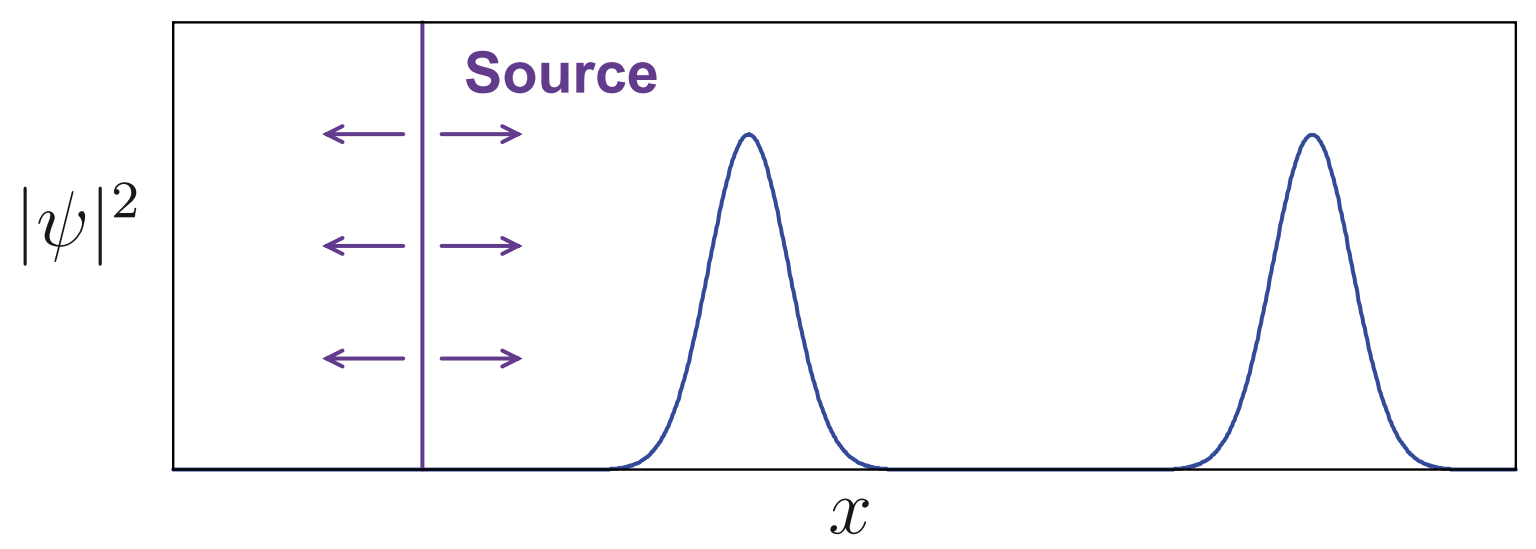

Realization with focused laser beams:

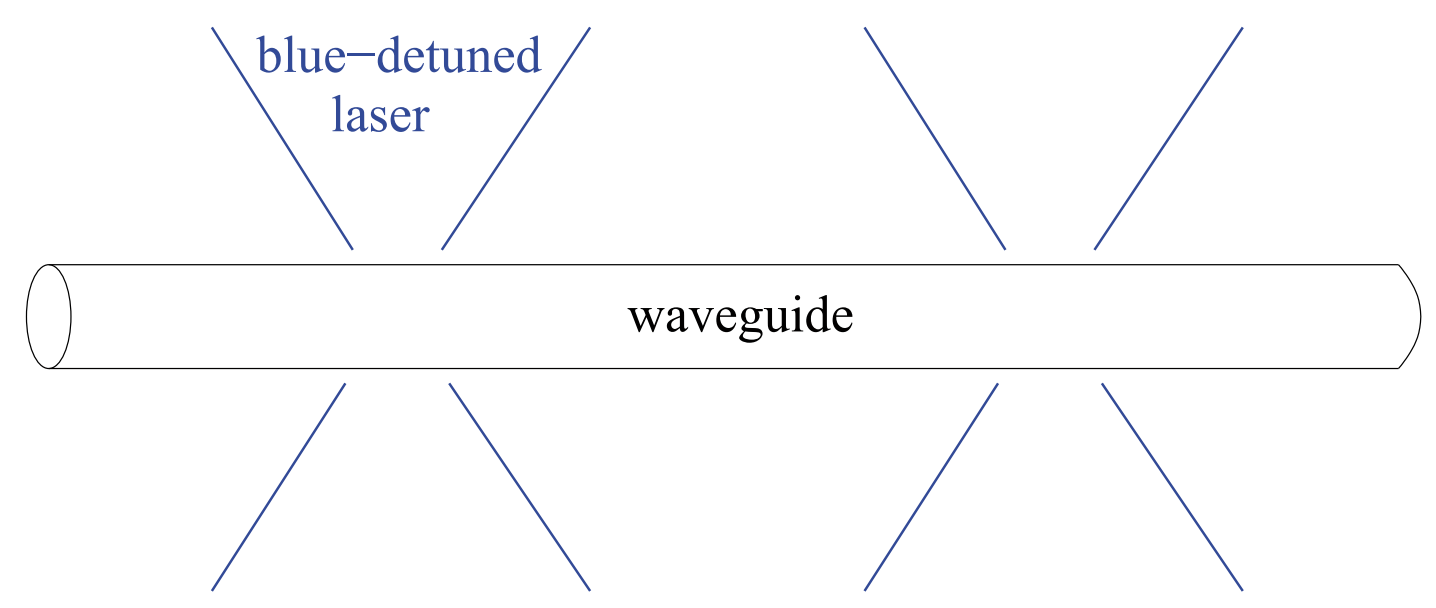




\section{Scattering through double barrier potentials}

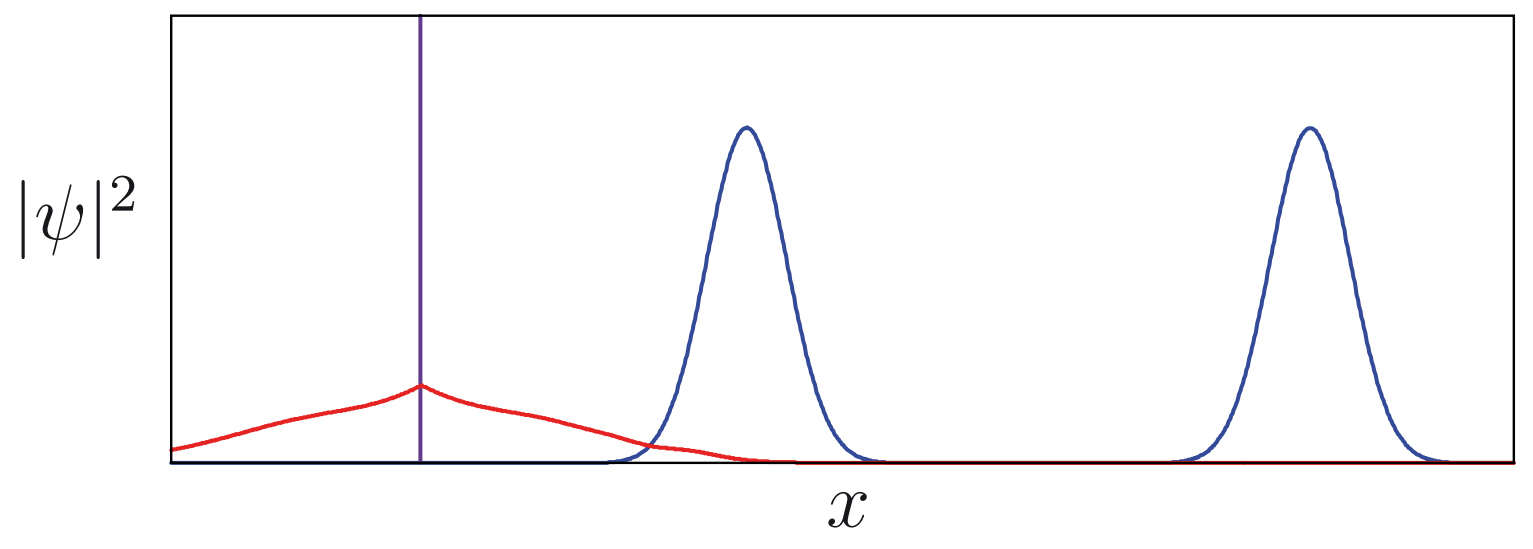




\section{Scattering through double barrier potentials}

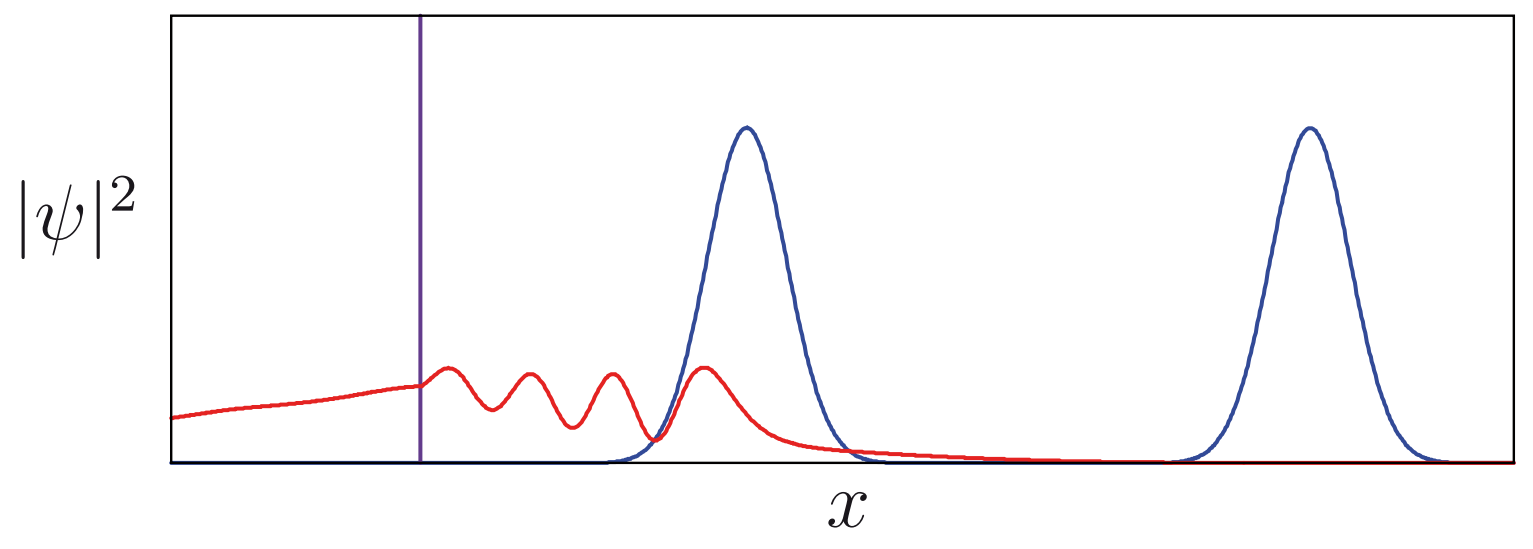




\section{Scattering through double barrier potentials}

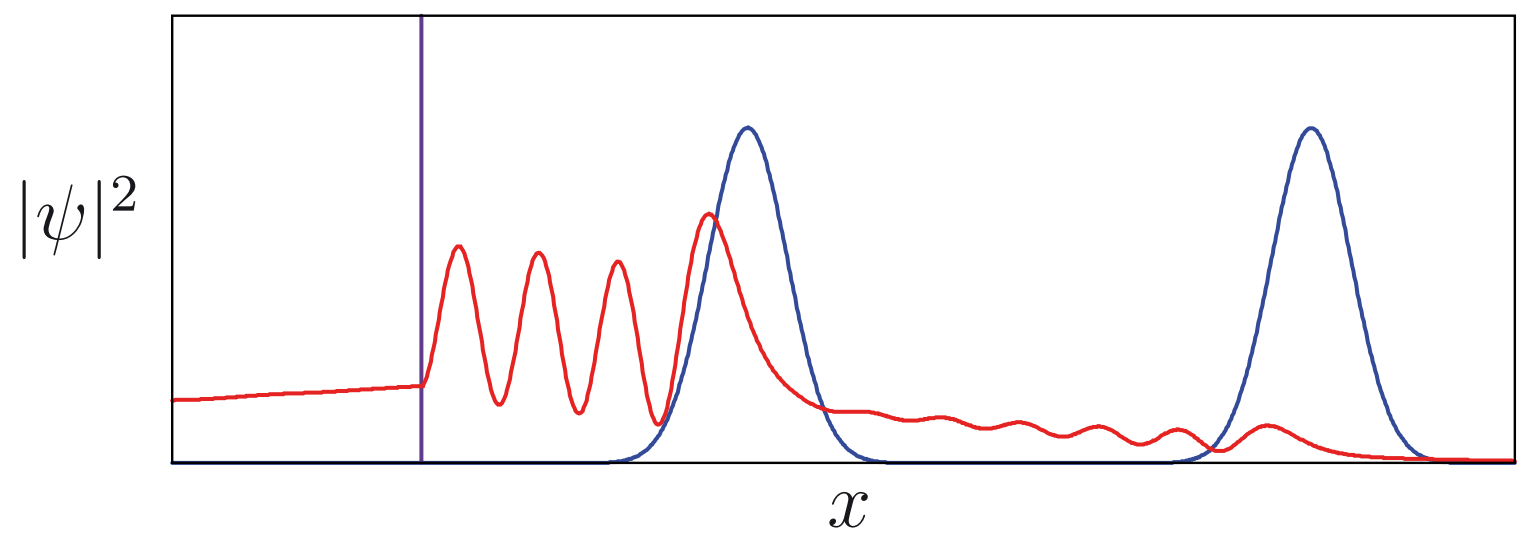




\section{Scattering through double barrier potentials}

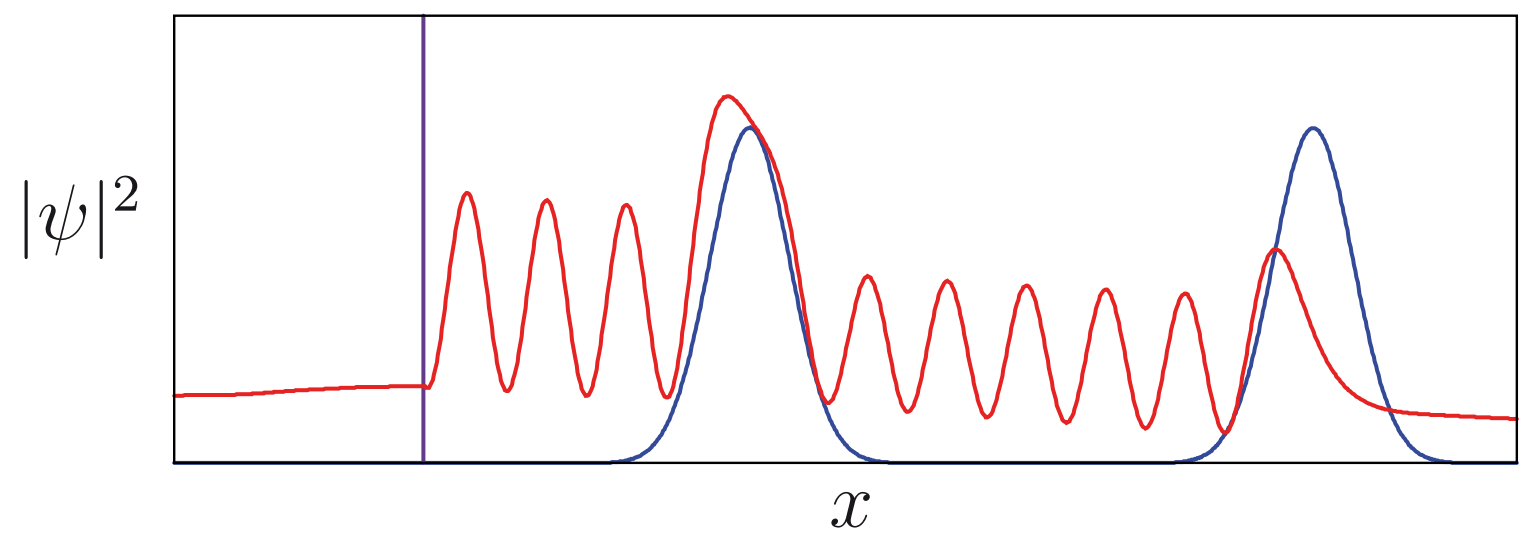




\section{Scattering through double barrier potentials}

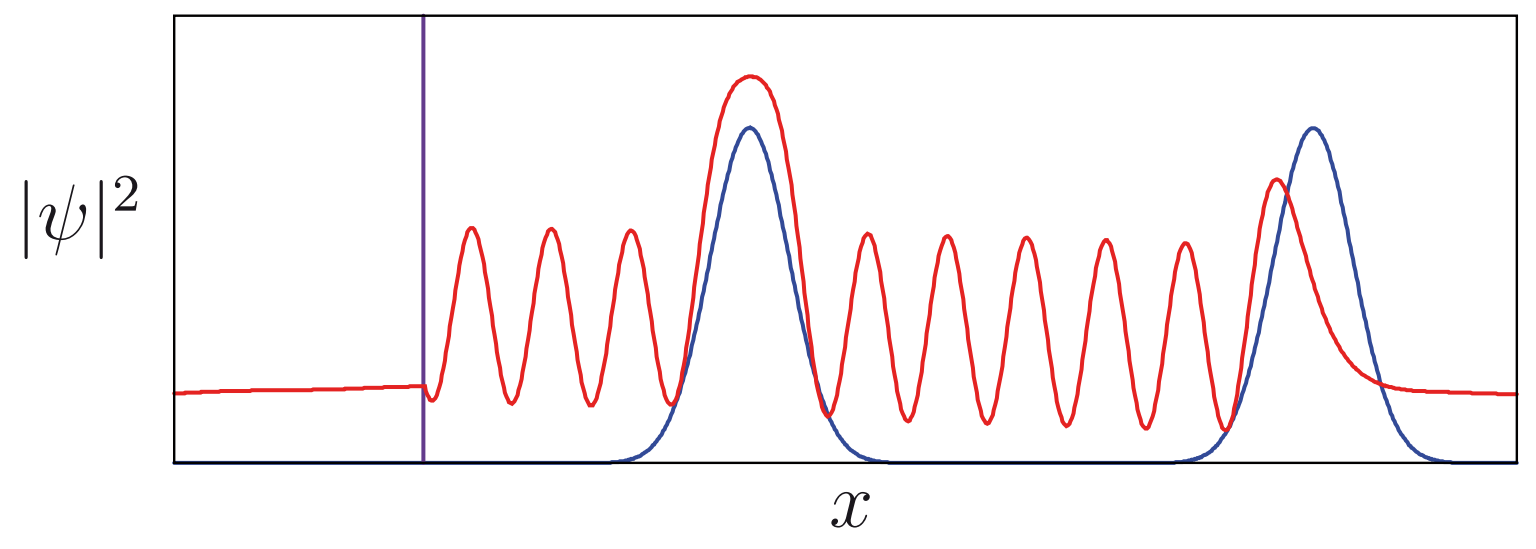




\section{Scattering through double barrier potentials}

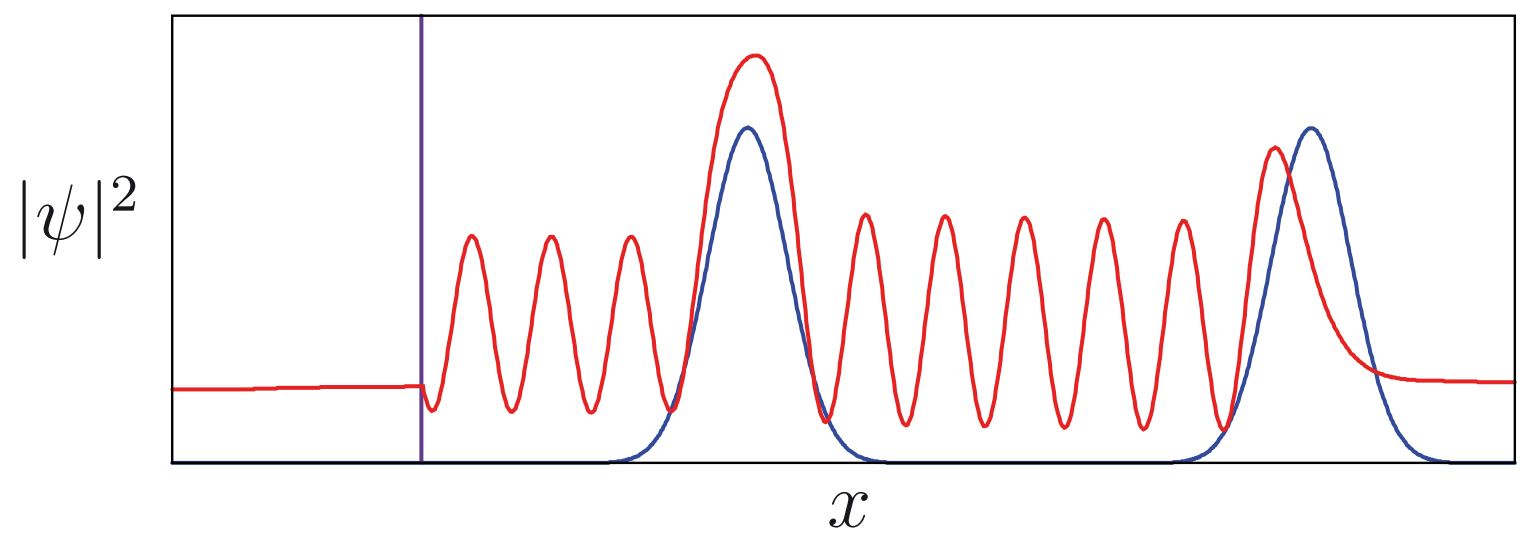




\section{Scattering through double barrier potentials}

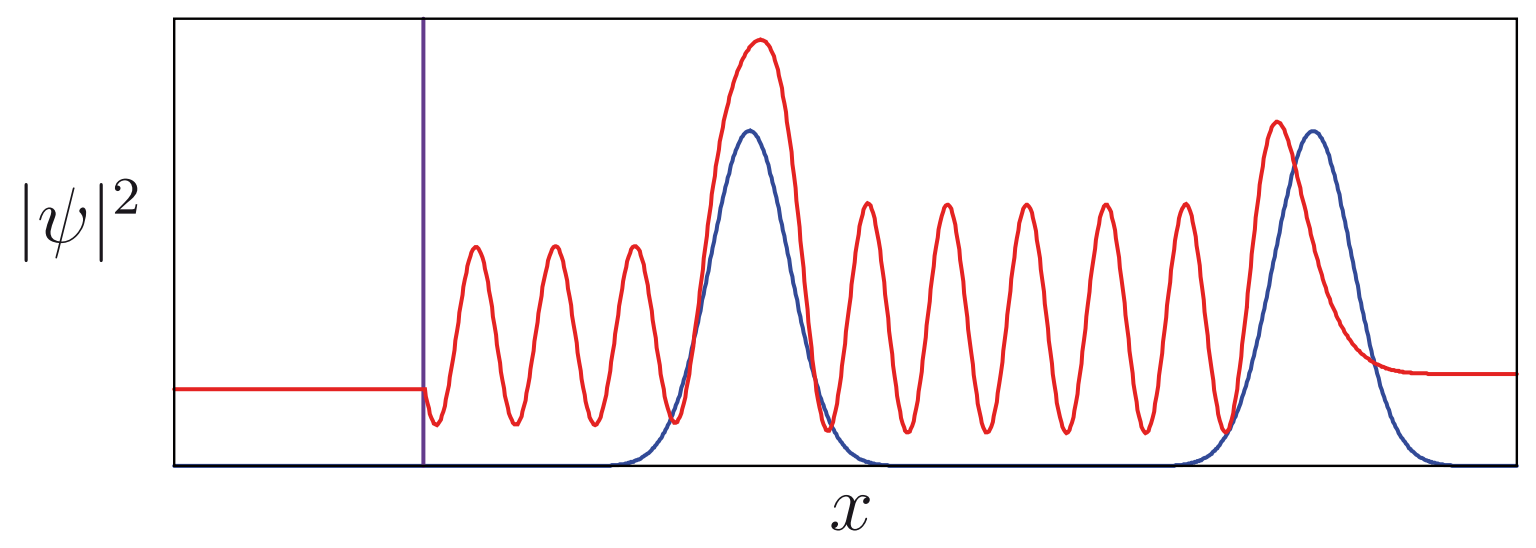




\section{Scattering through double barrier potentials}

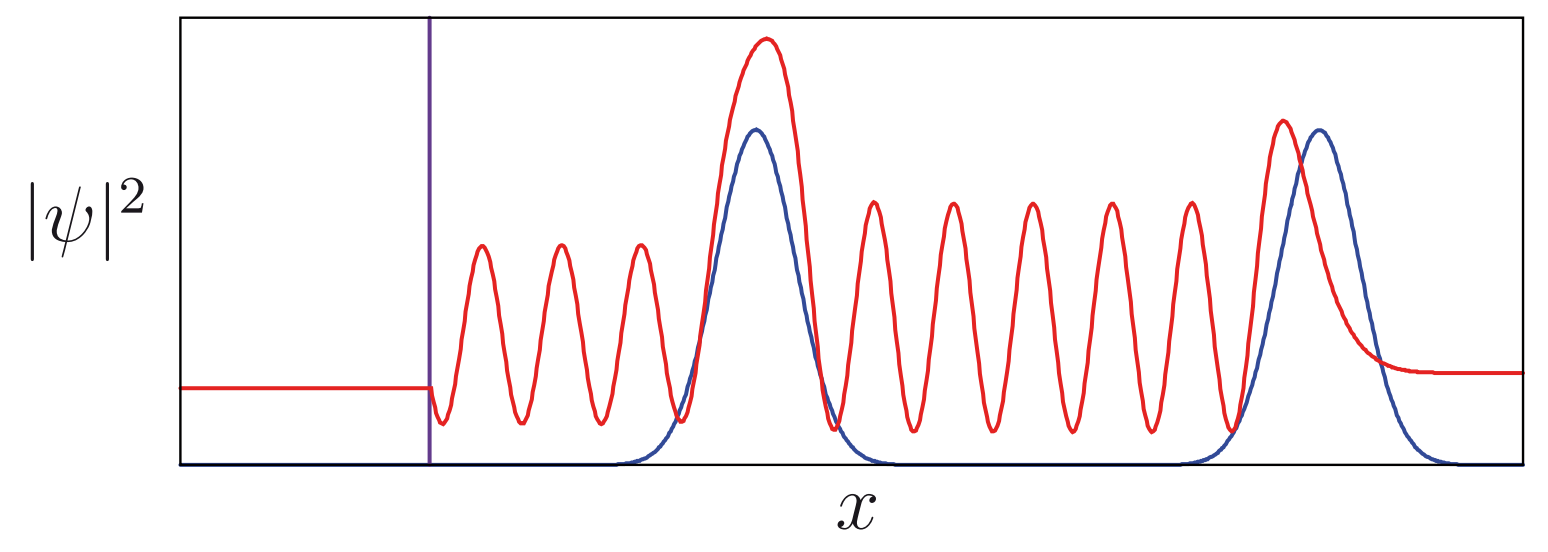

Transmission $=\frac{\text { total atomic current in the quasi-stationary regime }}{\text { incident current of the beam }(\rightarrow \text { source amplitude })}$ 


\section{Transmission of the condensate}

without interaction:

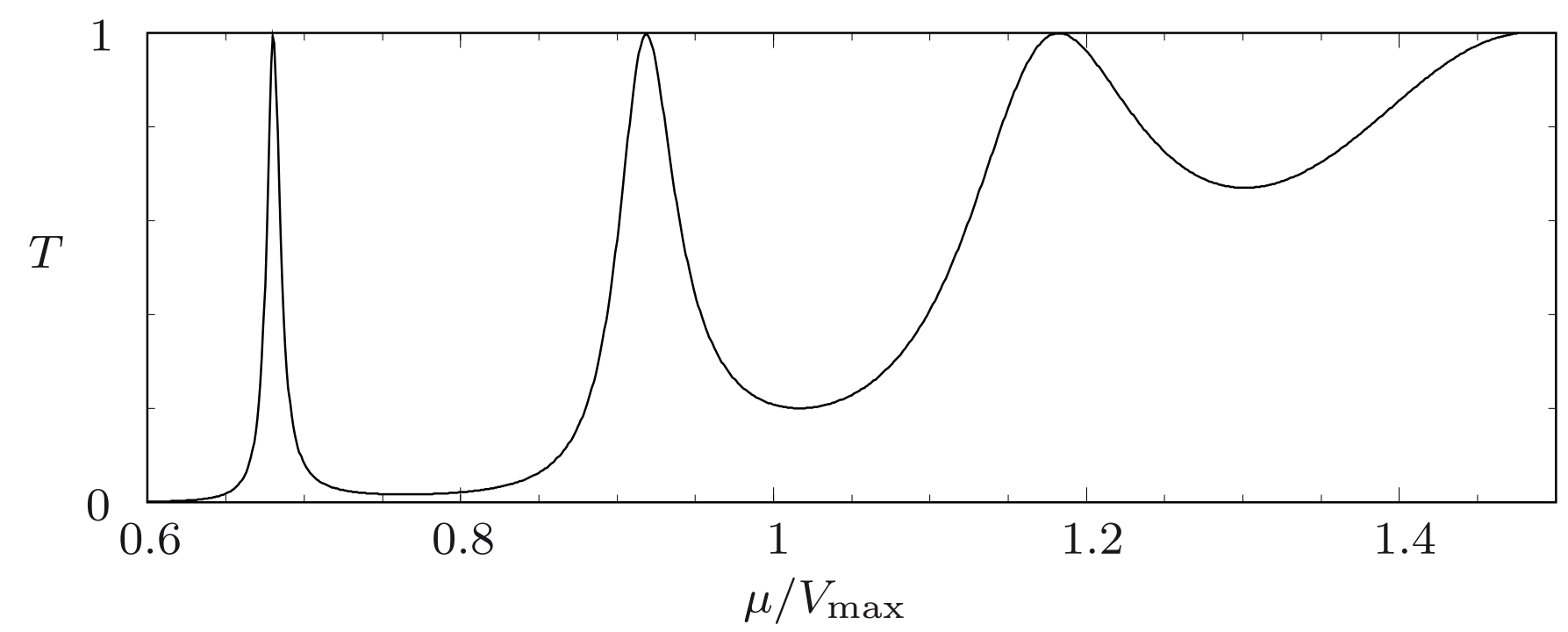

$\rightarrow$ resonant transmission through quasi-bound eigenstates

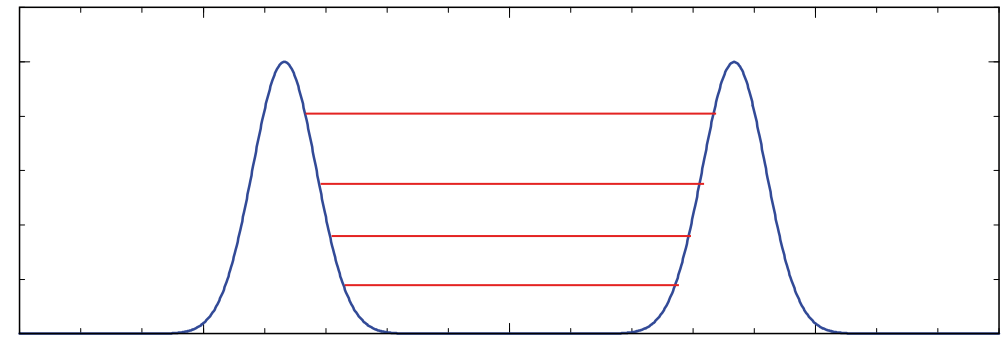




\section{Transmission of the condensate}

with interaction, at fixed incident current $\left(10^{4}\right.$ atoms/s)

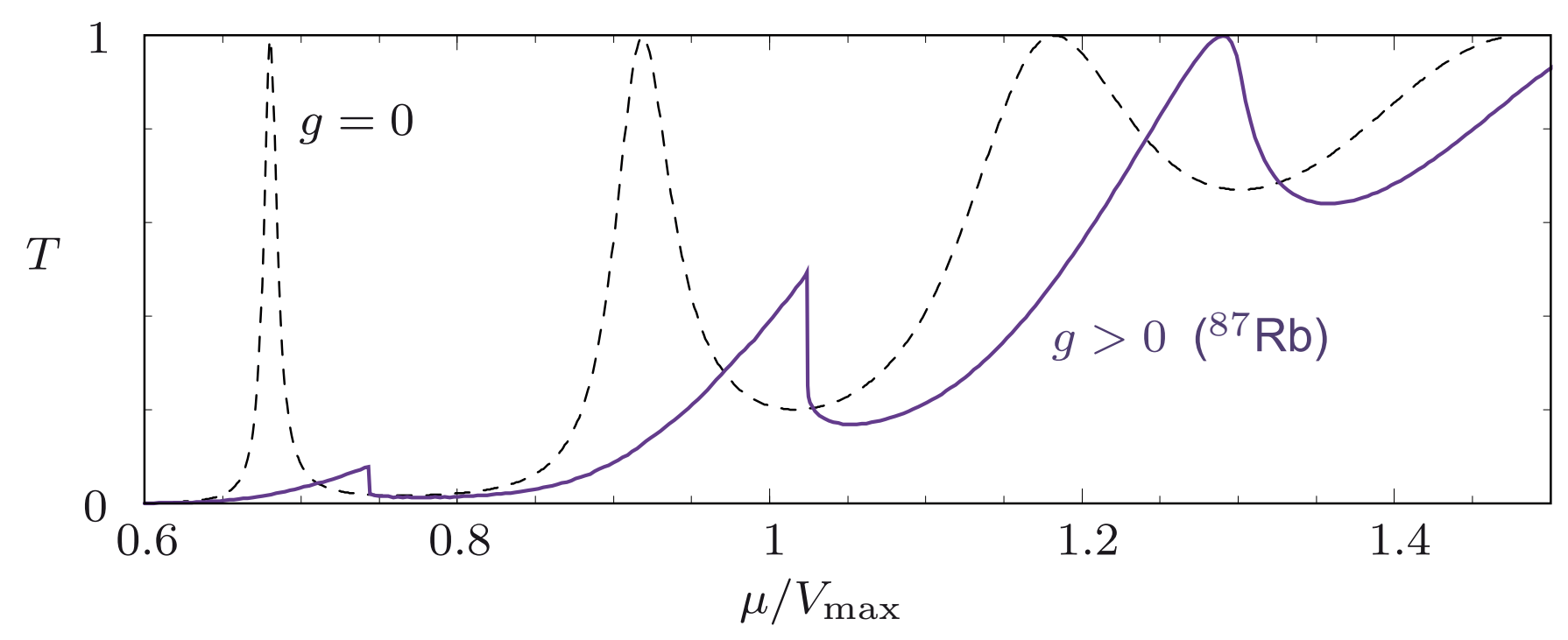




\section{Transmission of the condensate}

with interaction, at fixed incident current $\left(10^{4}\right.$ atoms/s)

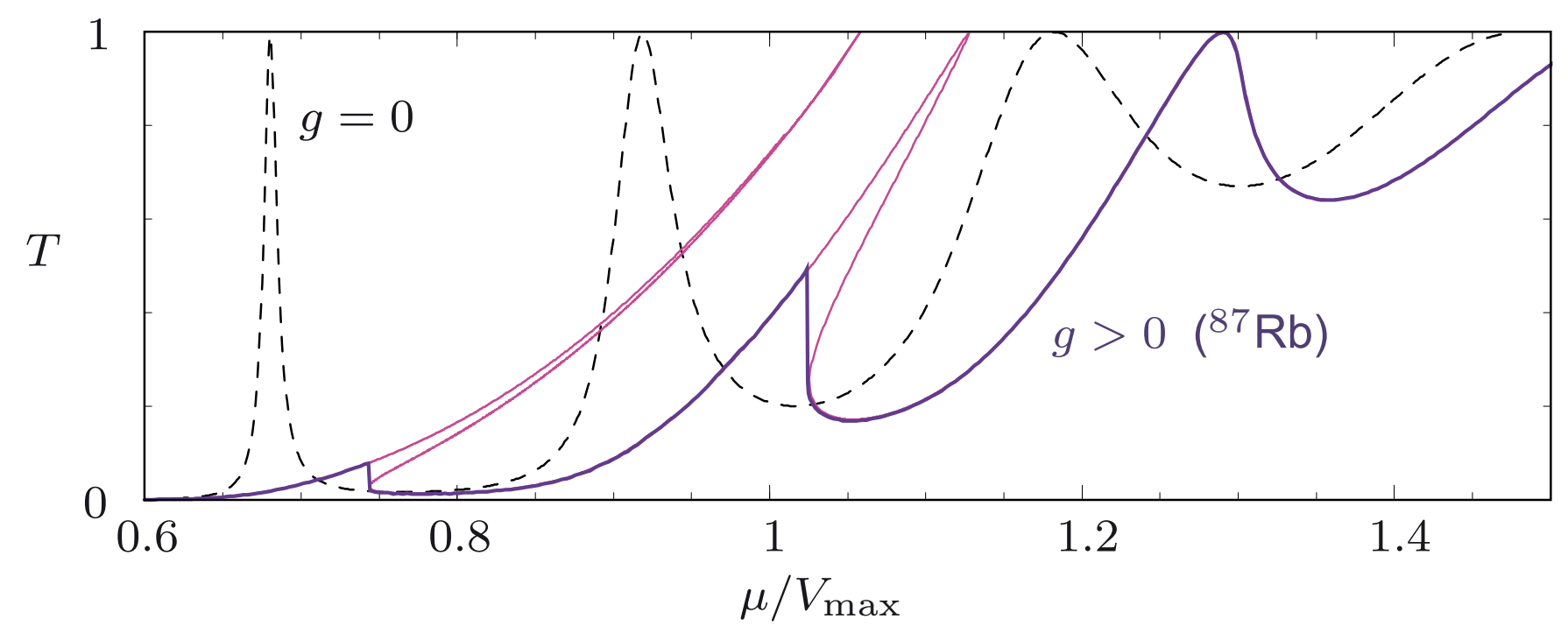

$\longrightarrow$ resonant transmission suppressed due to the interaction: nonlinear bistability phenomenon

T. Paul, K. Richter, and P.S., PRL 94, 020404 (2005) 


\section{Transmission through 2D confinement structures}

Example: circular billiard
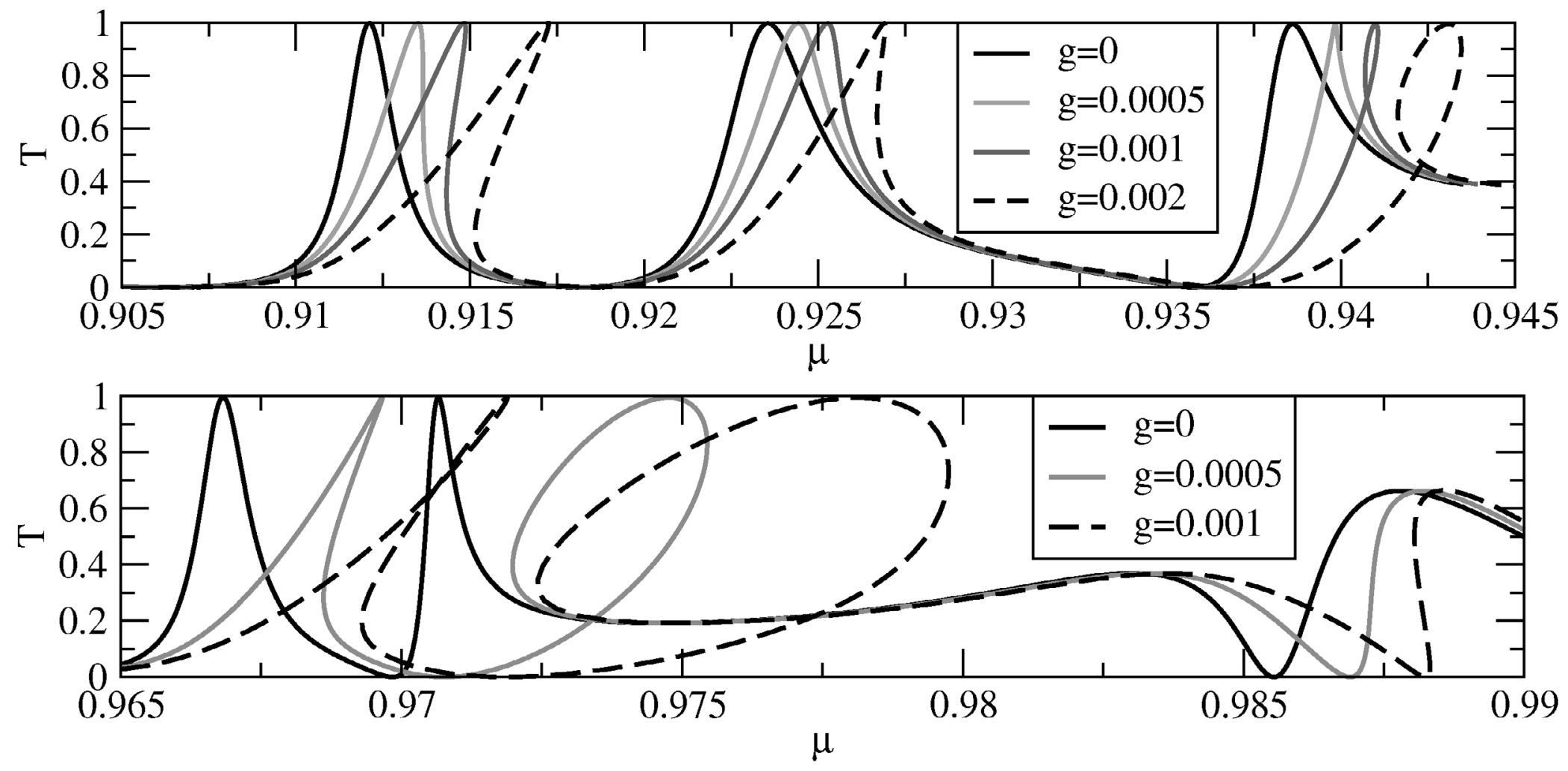

T. Hartmann et al., in preparation 


\section{Transport through longer scattering potentials}

Negligible interaction between the atoms:
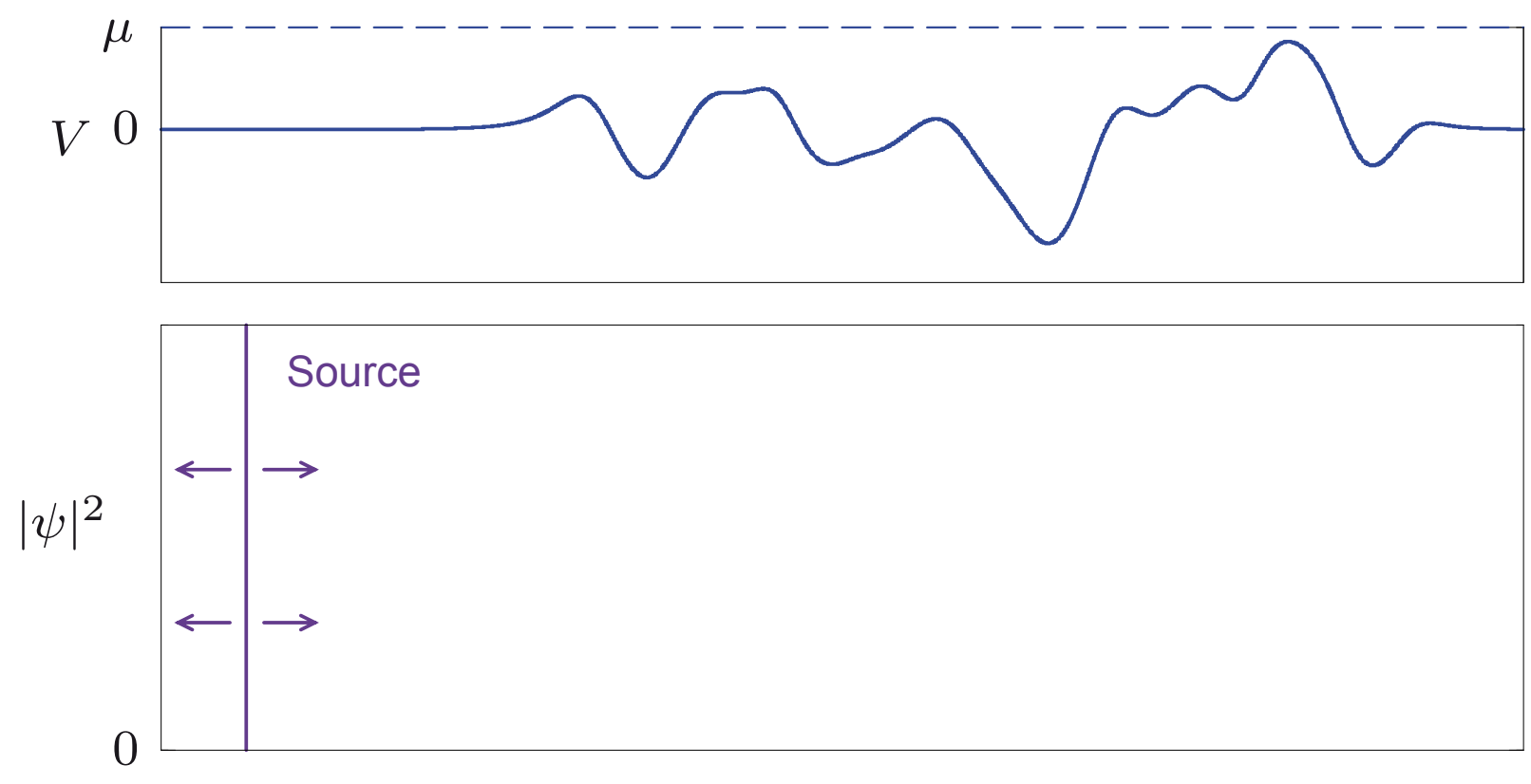


\section{Transport through longer scattering potentials}

Negligible interaction between the atoms:
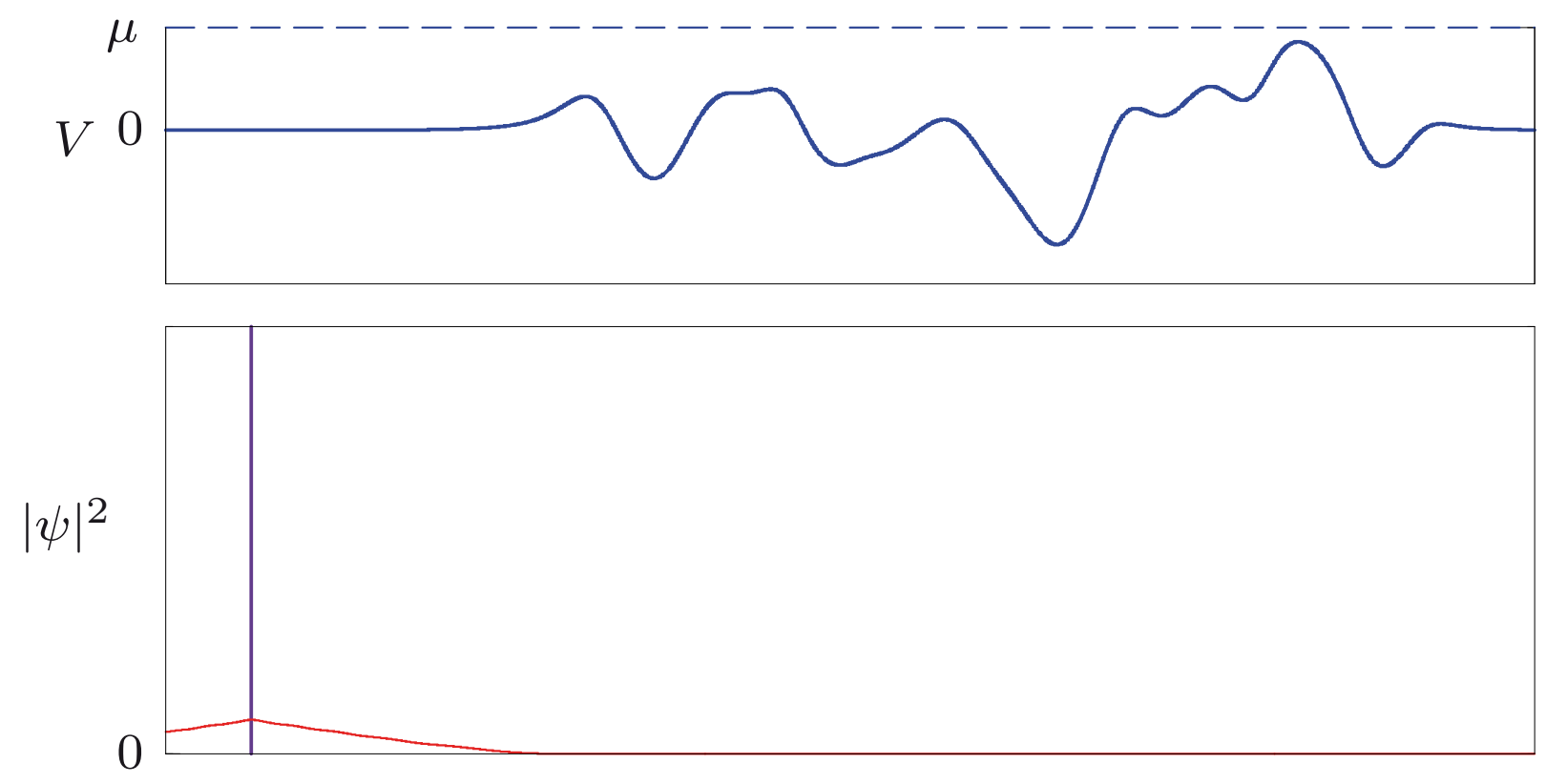


\section{Transport through longer scattering potentials}

Negligible interaction between the atoms:
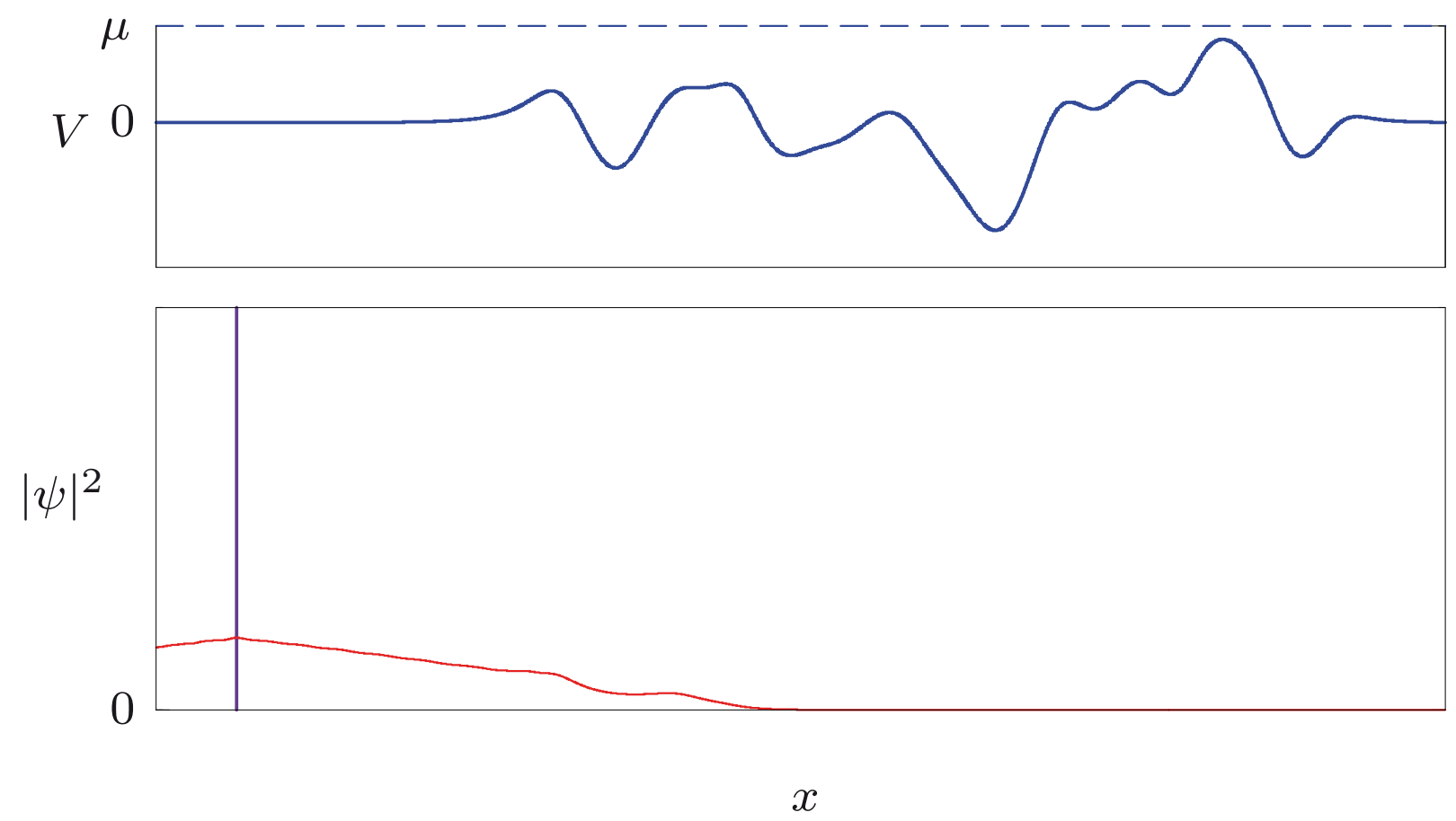


\section{Transport through longer scattering potentials}

Negligible interaction between the atoms:
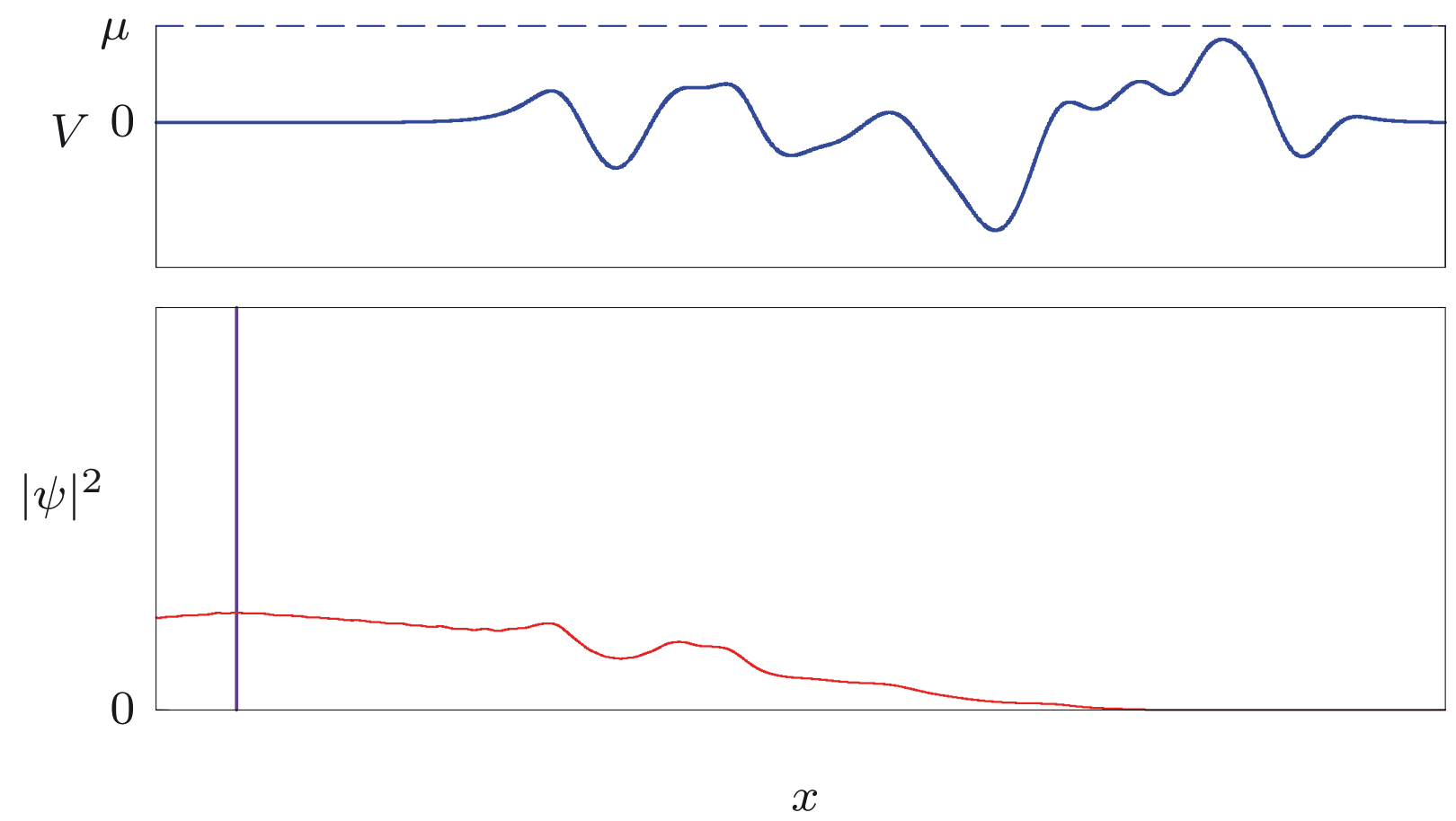


\section{Transport through longer scattering potentials}

Negligible interaction between the atoms:
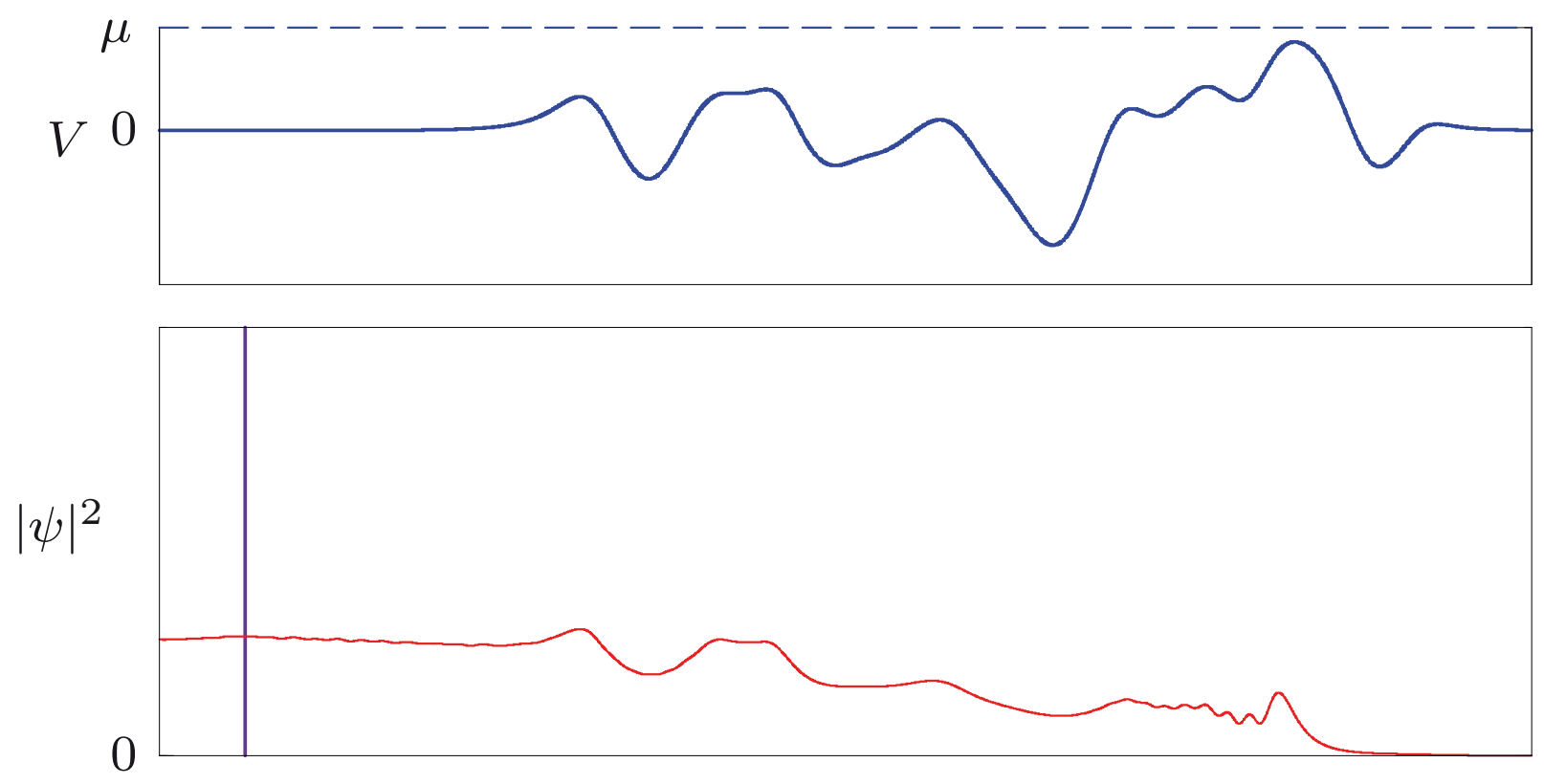

$x$ 


\section{Transport through longer scattering potentials}

Negligible interaction between the atoms:
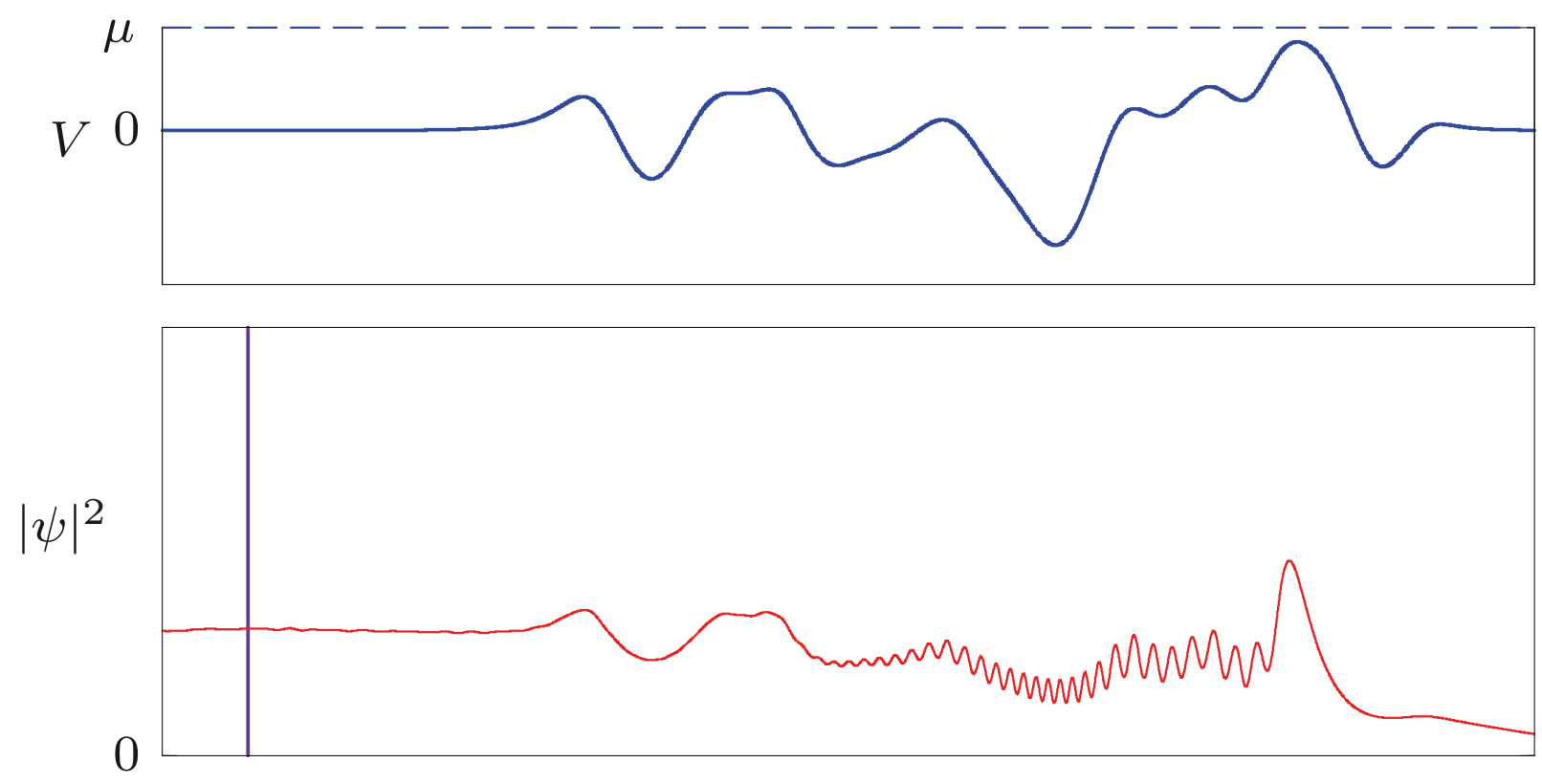

$x$ 


\section{Transport through longer scattering potentials}

Negligible interaction between the atoms:
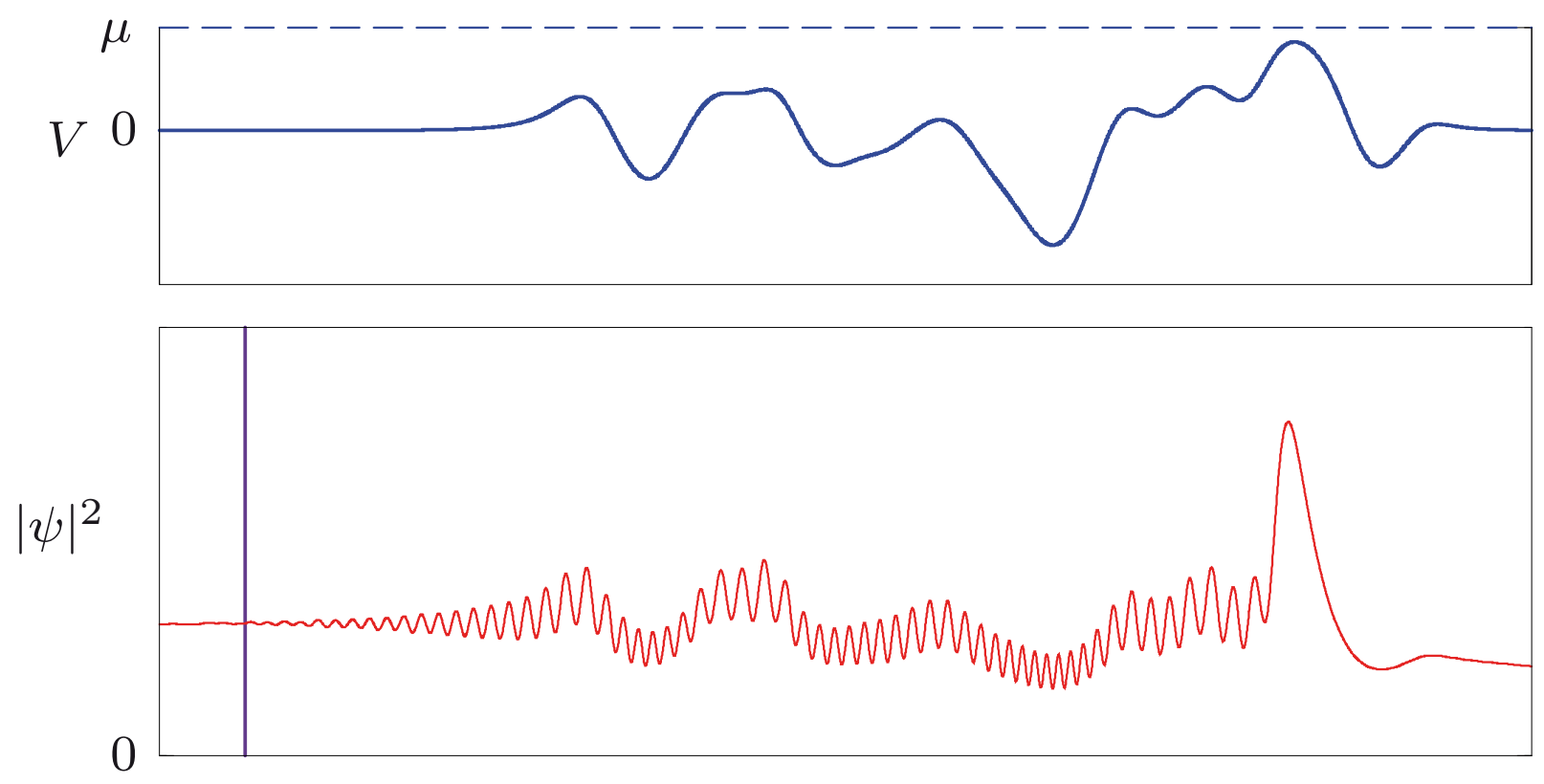

$x$ 


\section{Transport through longer scattering potentials}

Negligible interaction between the atoms:
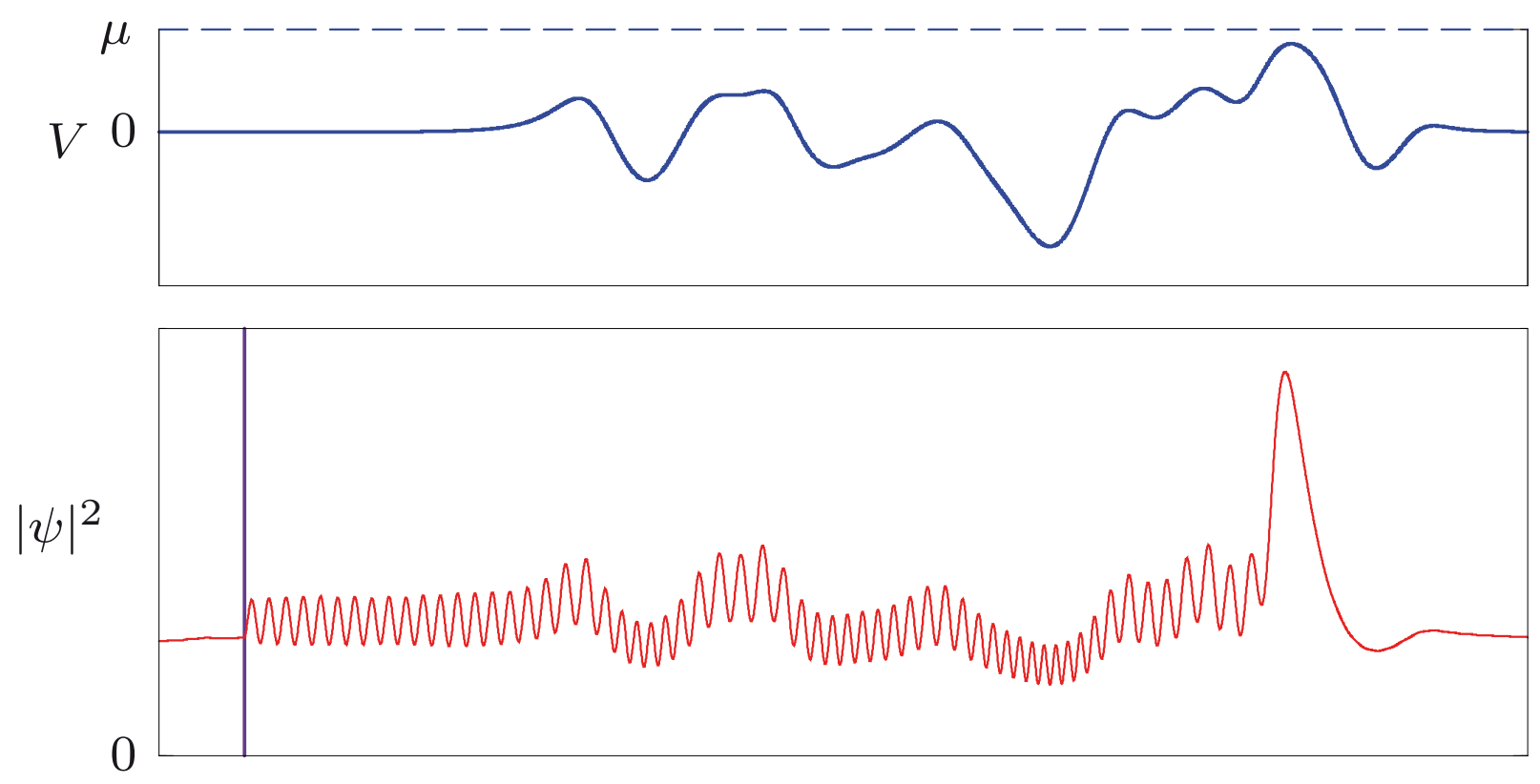

$x$ 


\section{Transport through longer scattering potentials}

Negligible interaction between the atoms:
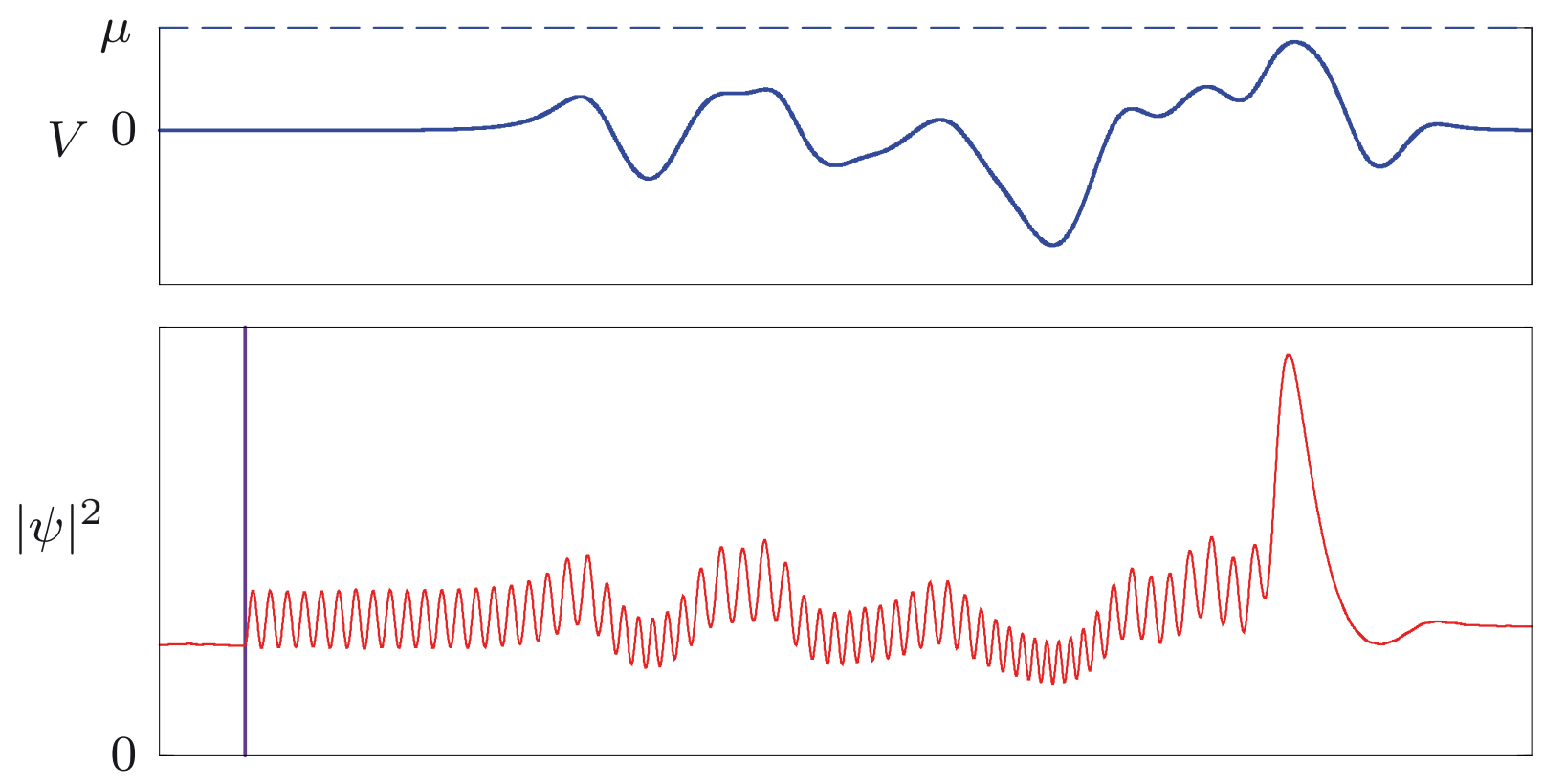

$x$ 


\section{Transport through longer scattering potentials}

Negligible interaction between the atoms:
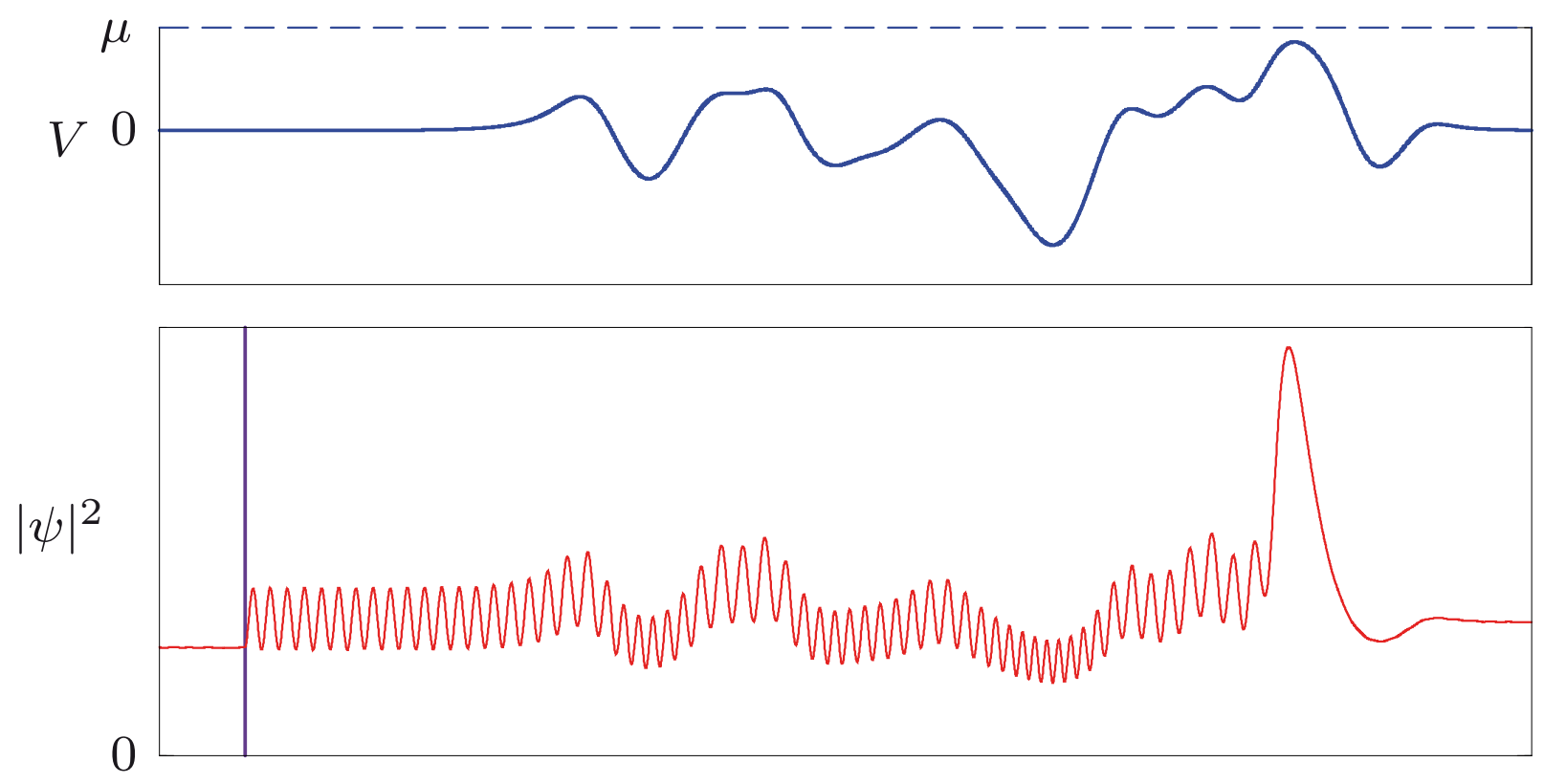

$x$ 


\section{Transport through longer scattering potentials}

Negligible interaction between the atoms:
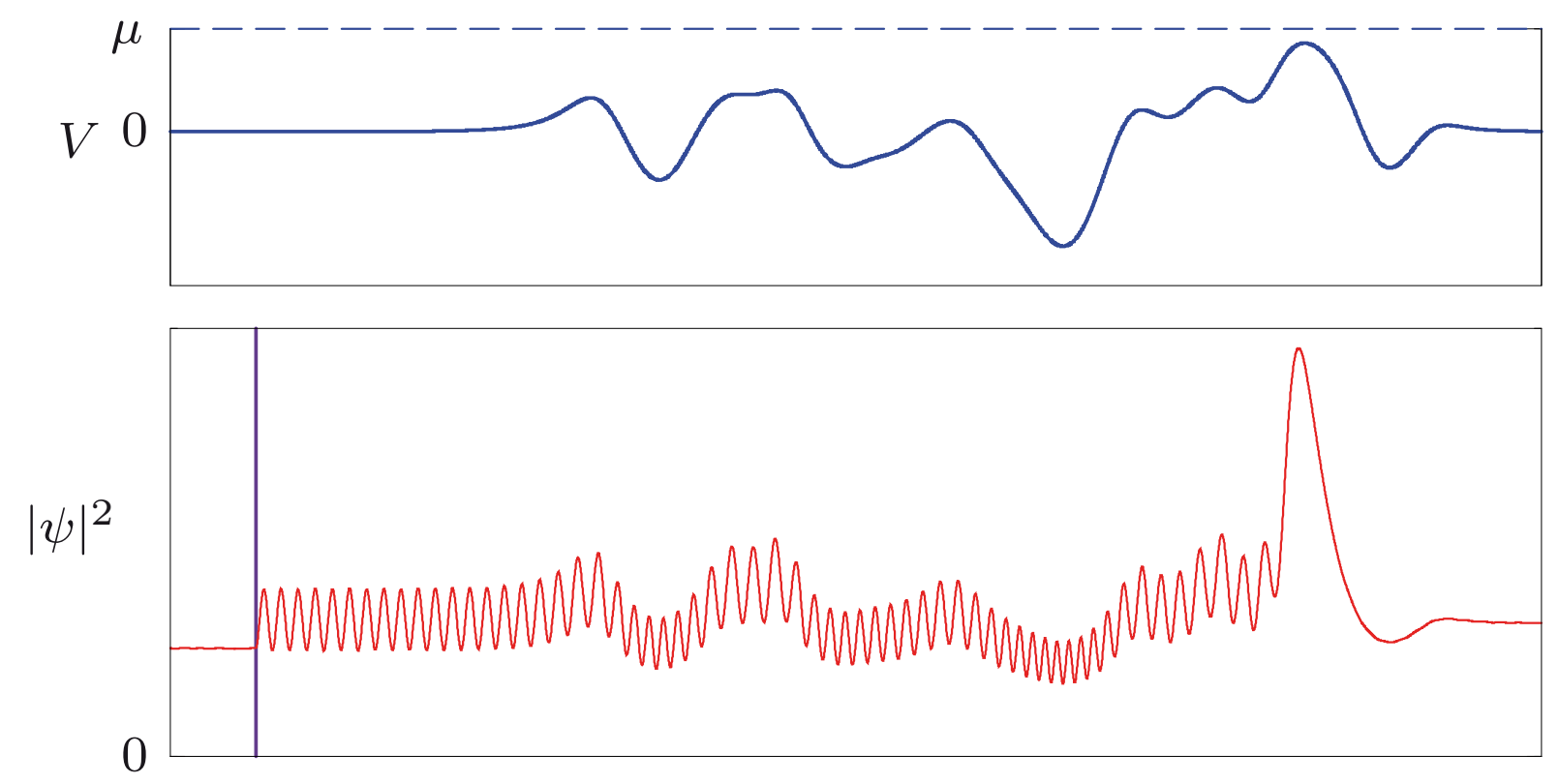

$\mathscr{X}$

$\longrightarrow$ stationary scattering state at the end of the propagation:

$$
|\psi(x)|^{2} \propto 1 / \sqrt{2 m[\mu-V(x)]}
$$




\section{Transport through longer scattering potentials}

Finite interaction between the atoms: $g|\psi|^{2} \simeq 0.1 \mu$
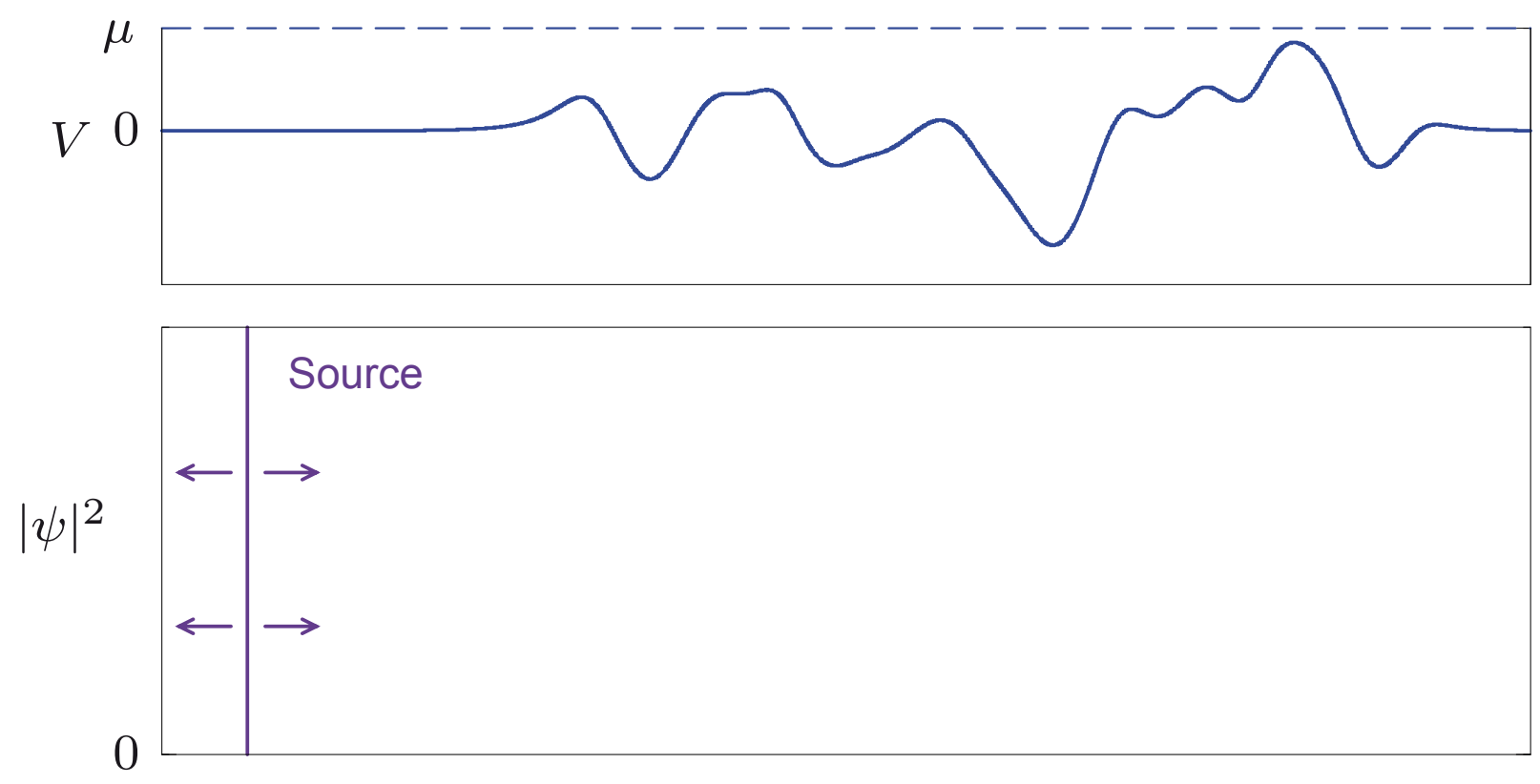


\section{Transport through longer scattering potentials}

Finite interaction between the atoms: $g|\psi|^{2} \simeq 0.1 \mu$
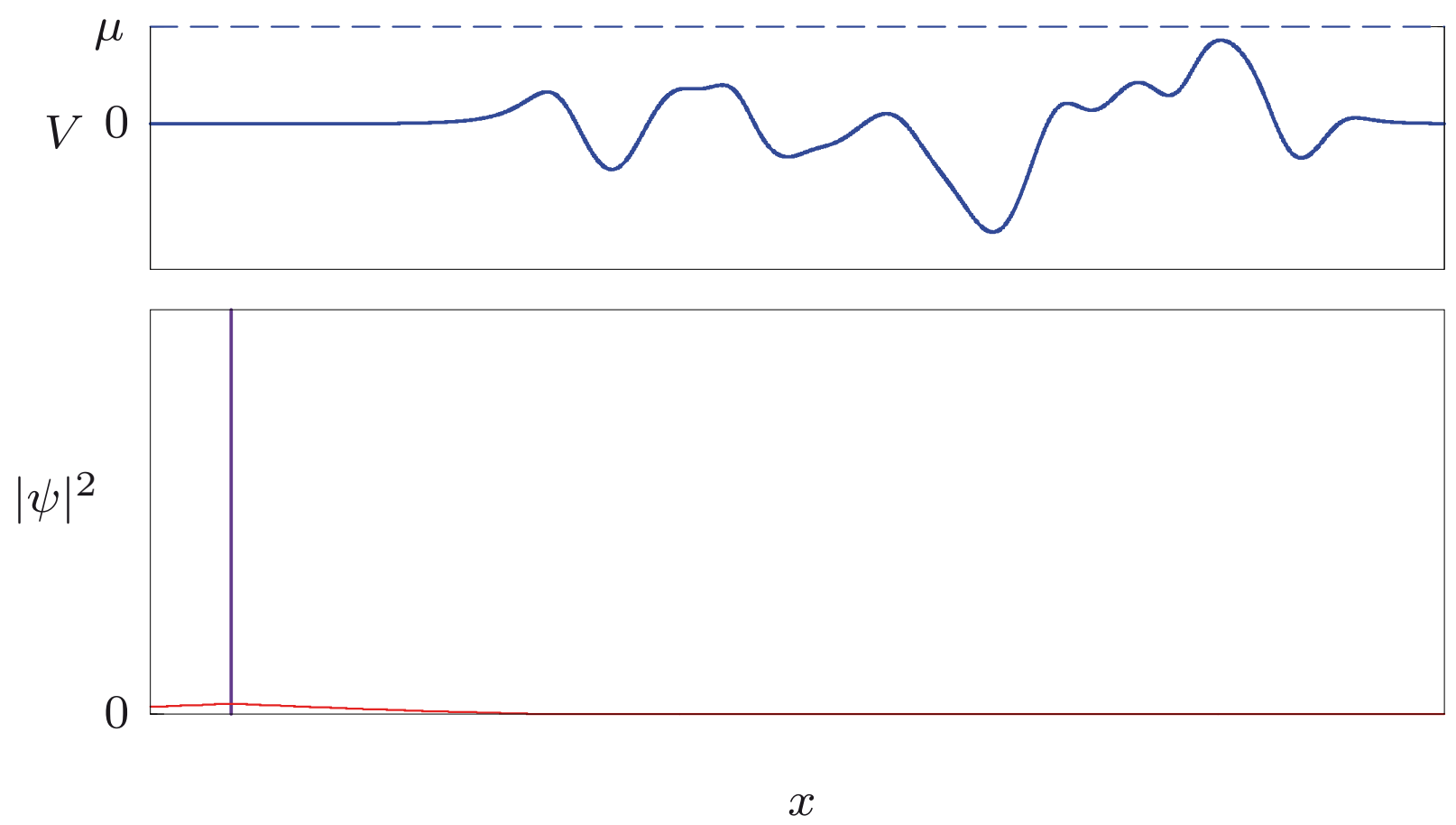


\section{Transport through longer scattering potentials}

Finite interaction between the atoms: $g|\psi|^{2} \simeq 0.1 \mu$
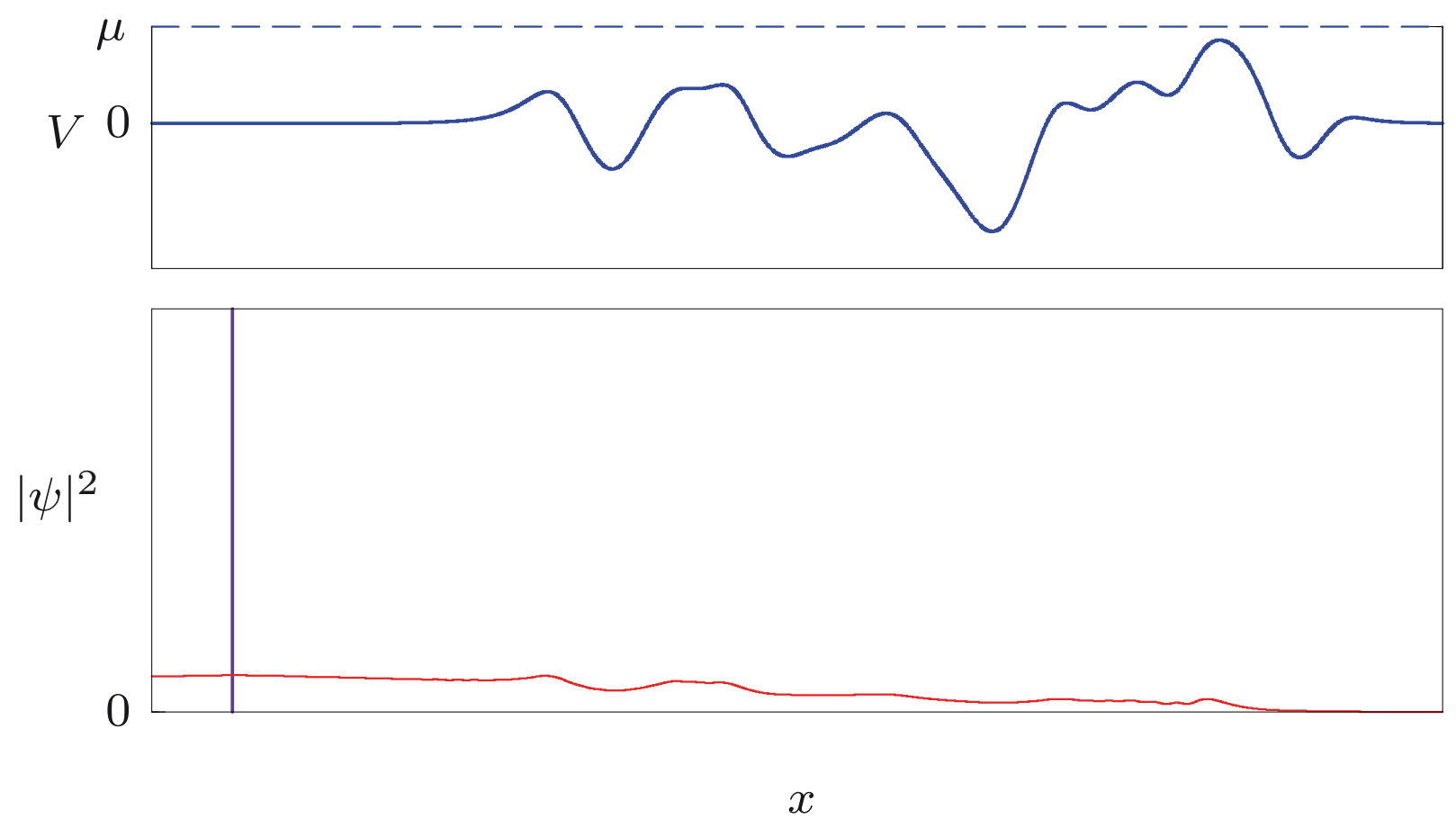


\section{Transport through longer scattering potentials}

Finite interaction between the atoms: $g|\psi|^{2} \simeq 0.1 \mu$
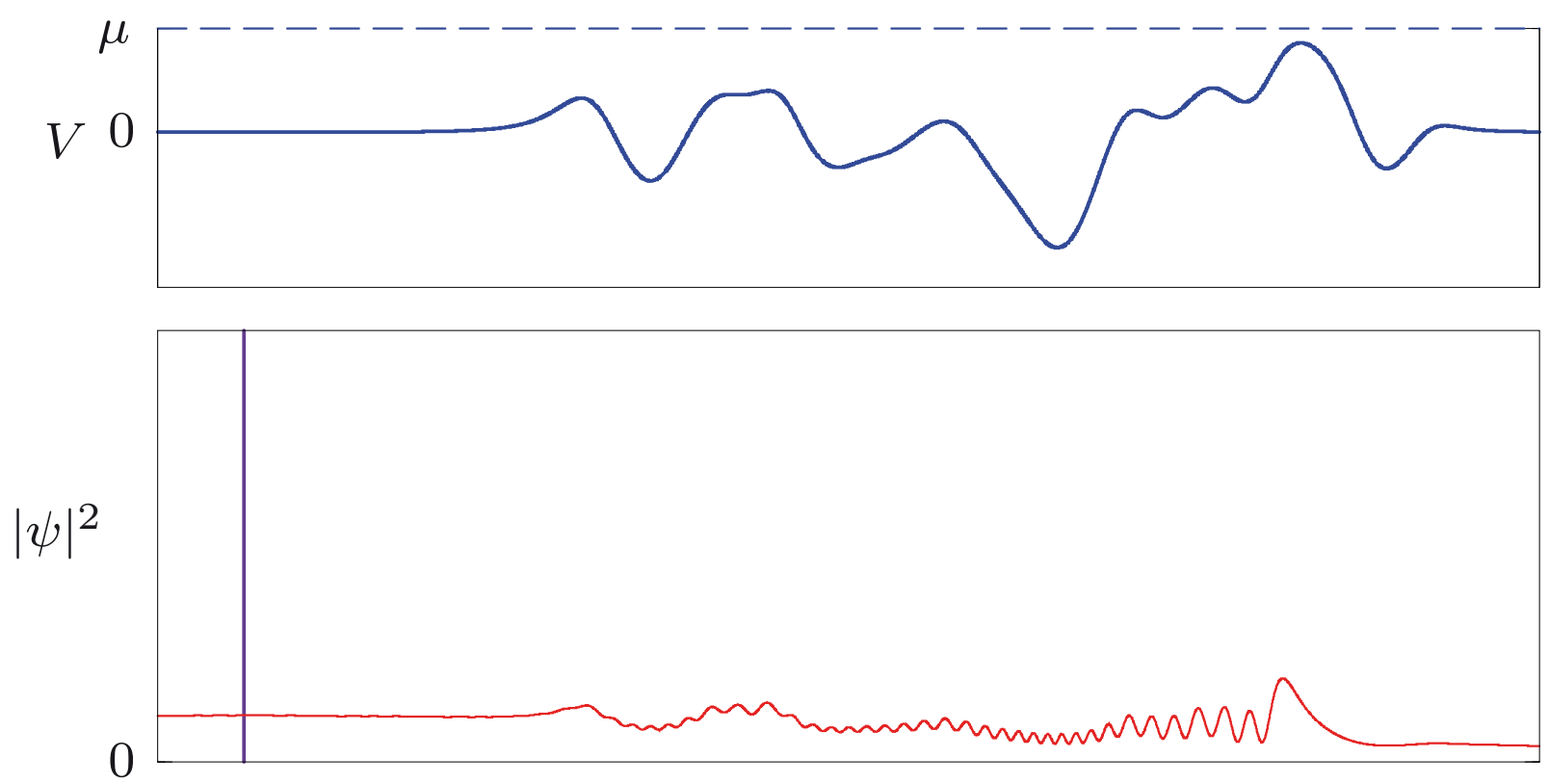

$x$ 


\section{Transport through longer scattering potentials}

Finite interaction between the atoms: $g|\psi|^{2} \simeq 0.1 \mu$
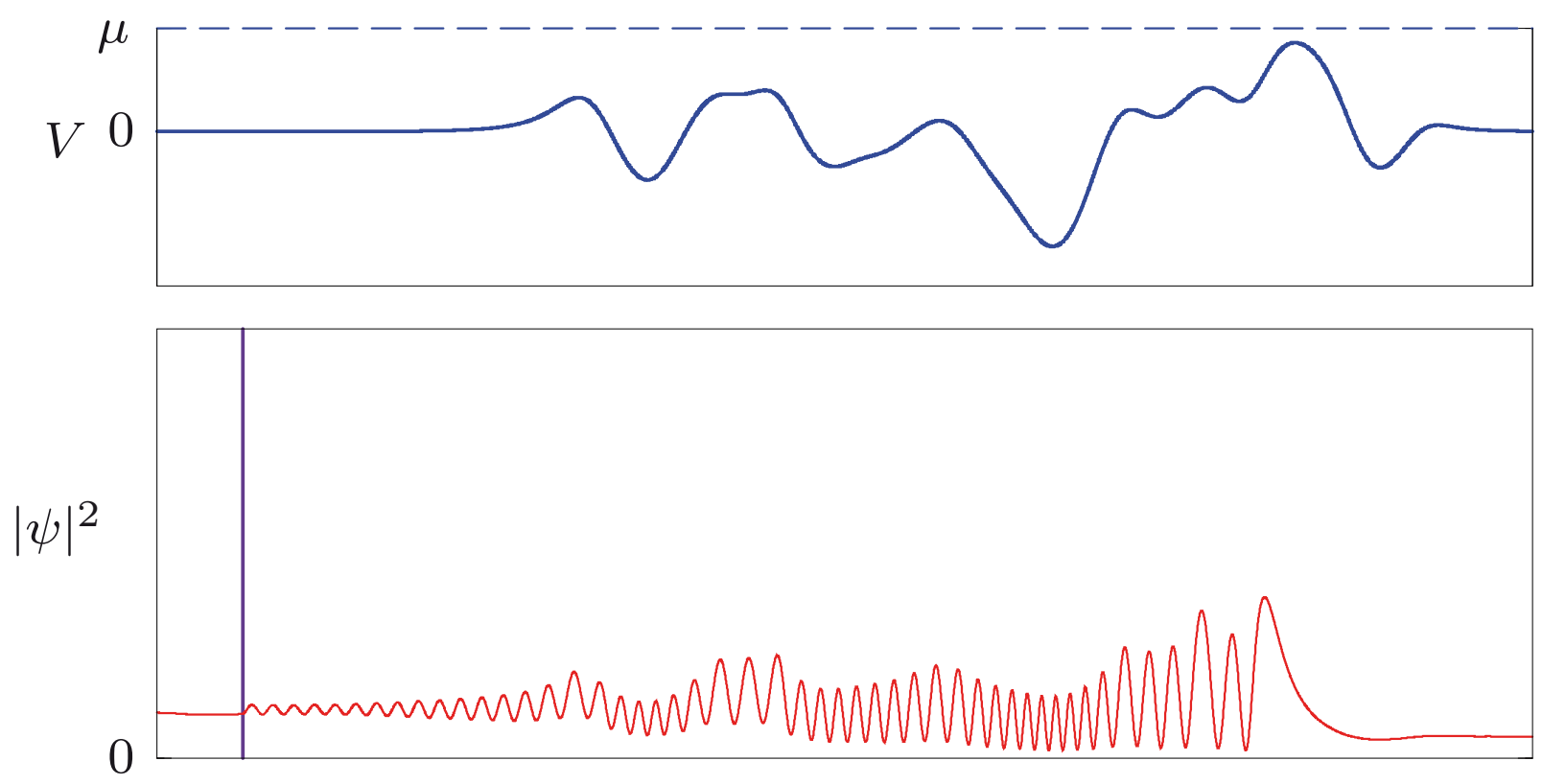

$x$ 


\section{Transport through longer scattering potentials}

Finite interaction between the atoms: $g|\psi|^{2} \simeq 0.1 \mu$
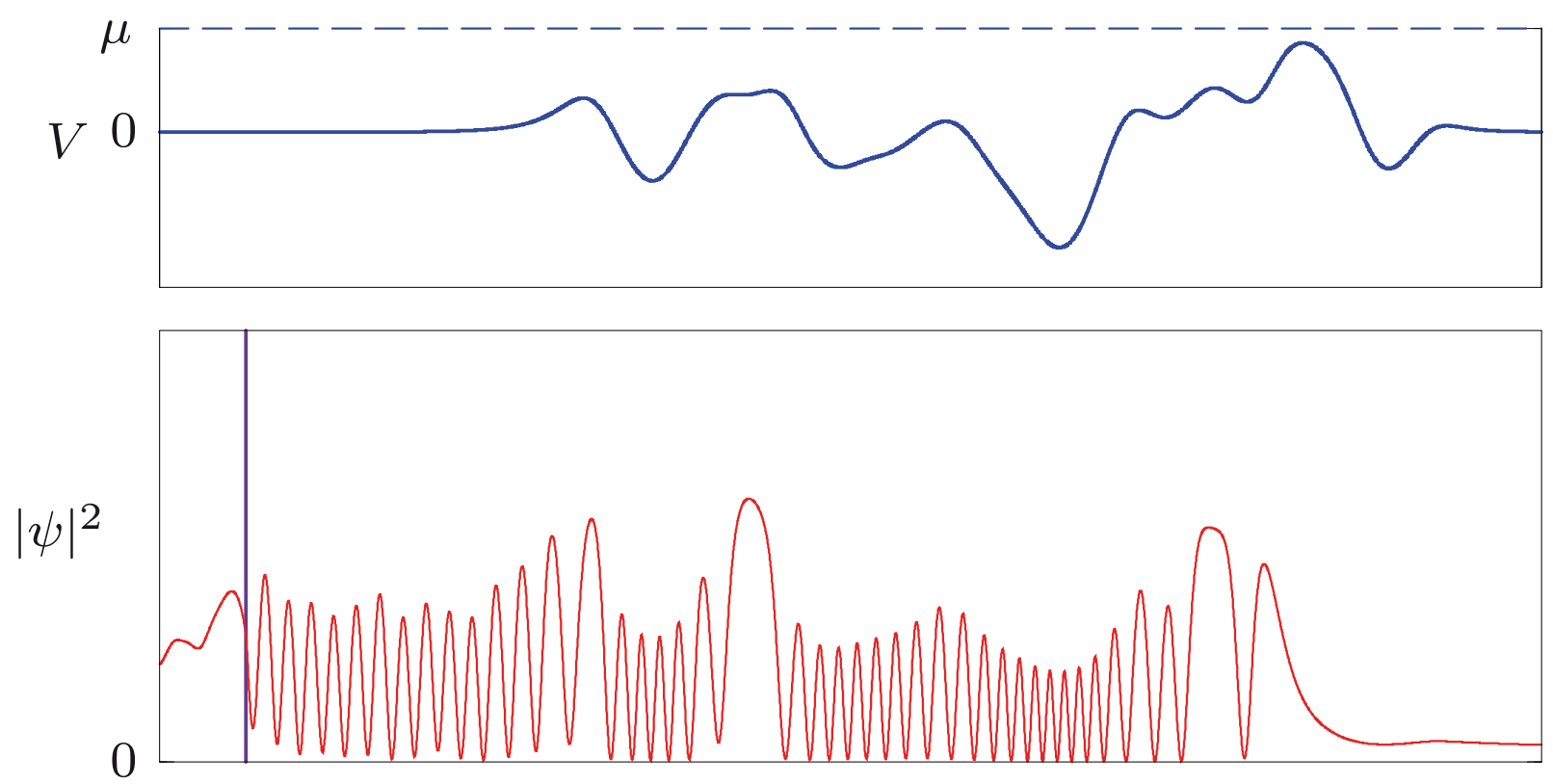


\section{Transport through longer scattering potentials}

Finite interaction between the atoms: $g|\psi|^{2} \simeq 0.1 \mu$
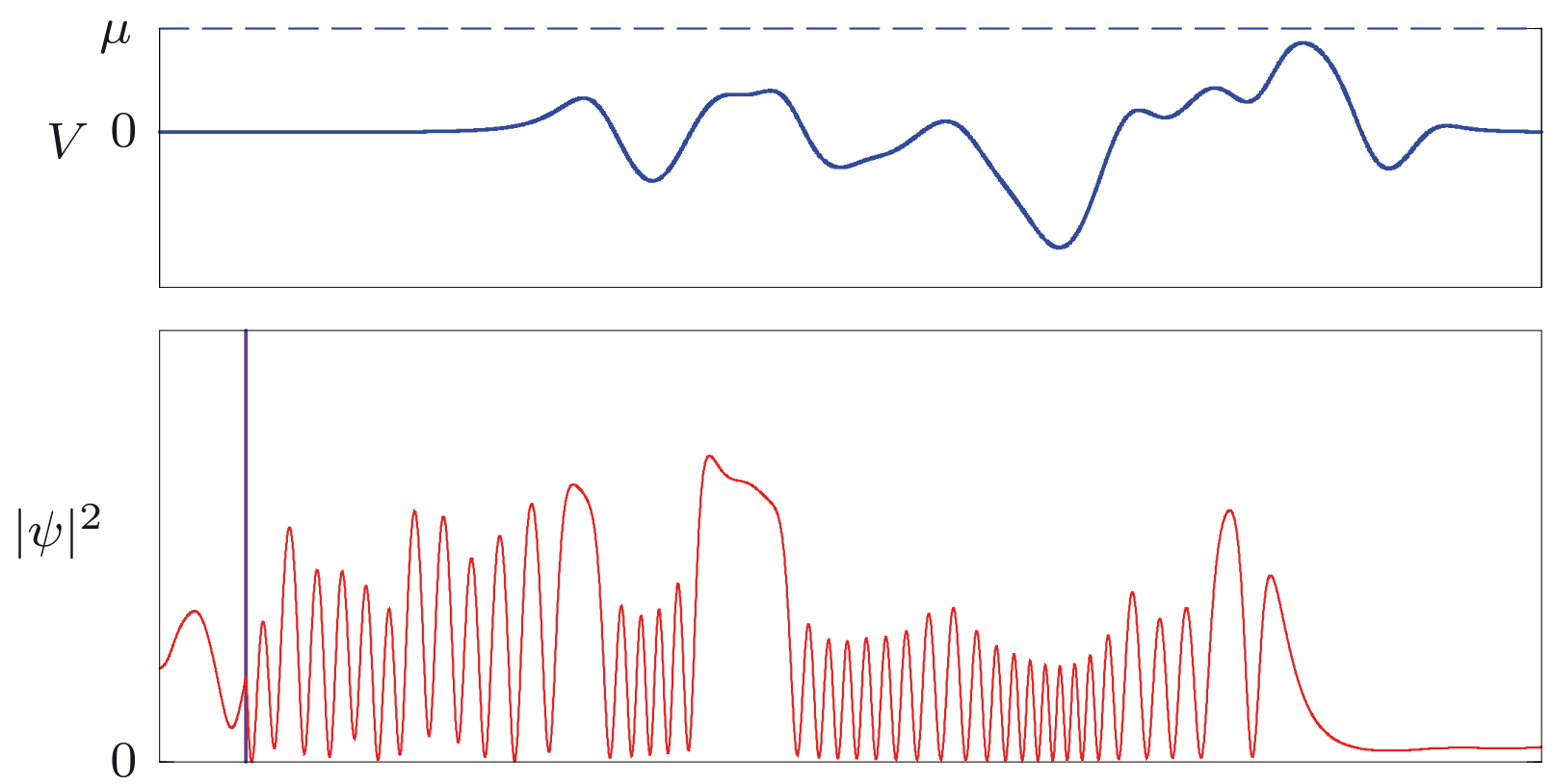


\section{Transport through longer scattering potentials}

Finite interaction between the atoms: $g|\psi|^{2} \simeq 0.1 \mu$
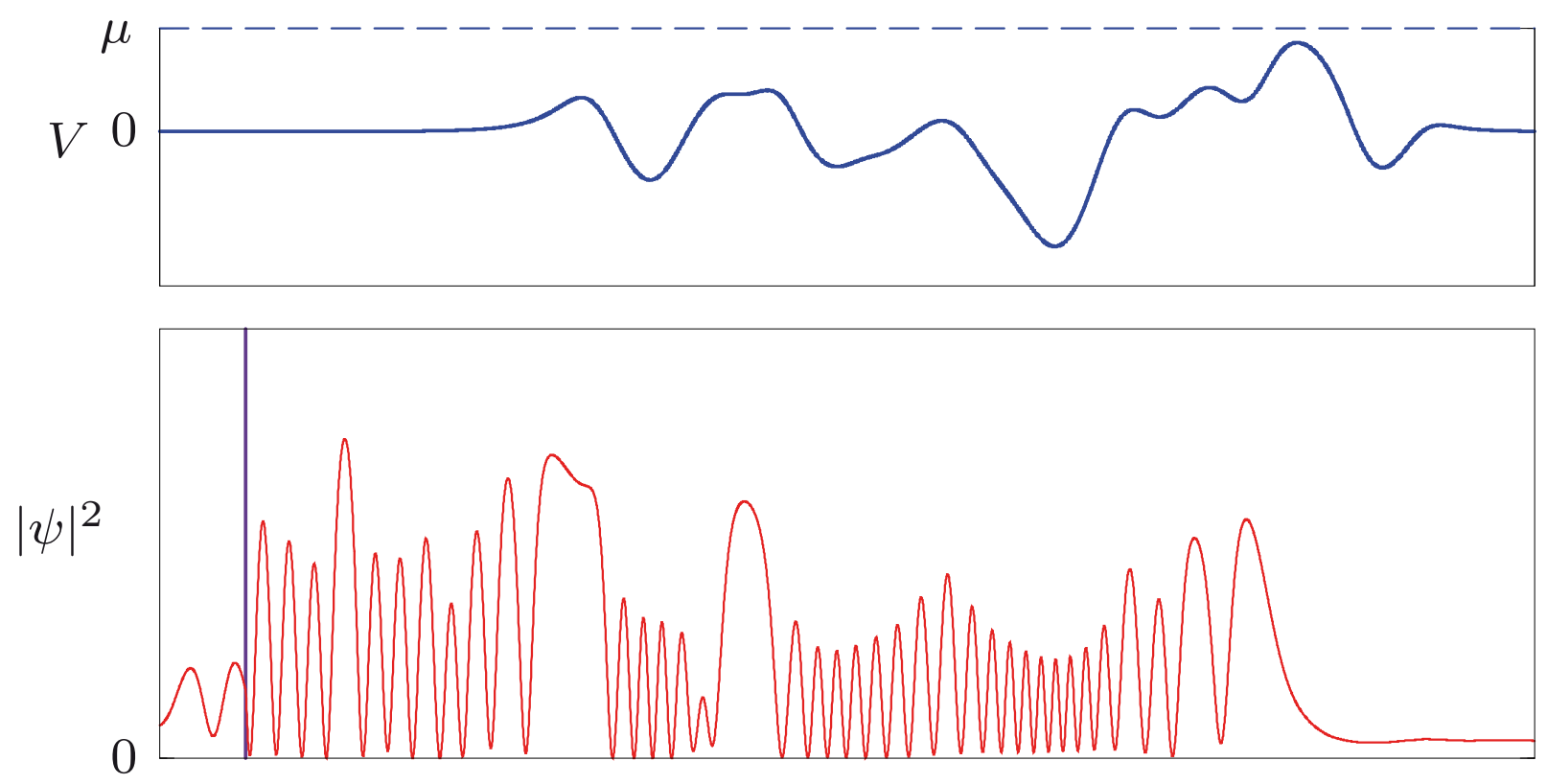


\section{Transport through longer scattering potentials}

Finite interaction between the atoms: $g|\psi|^{2} \simeq 0.1 \mu$
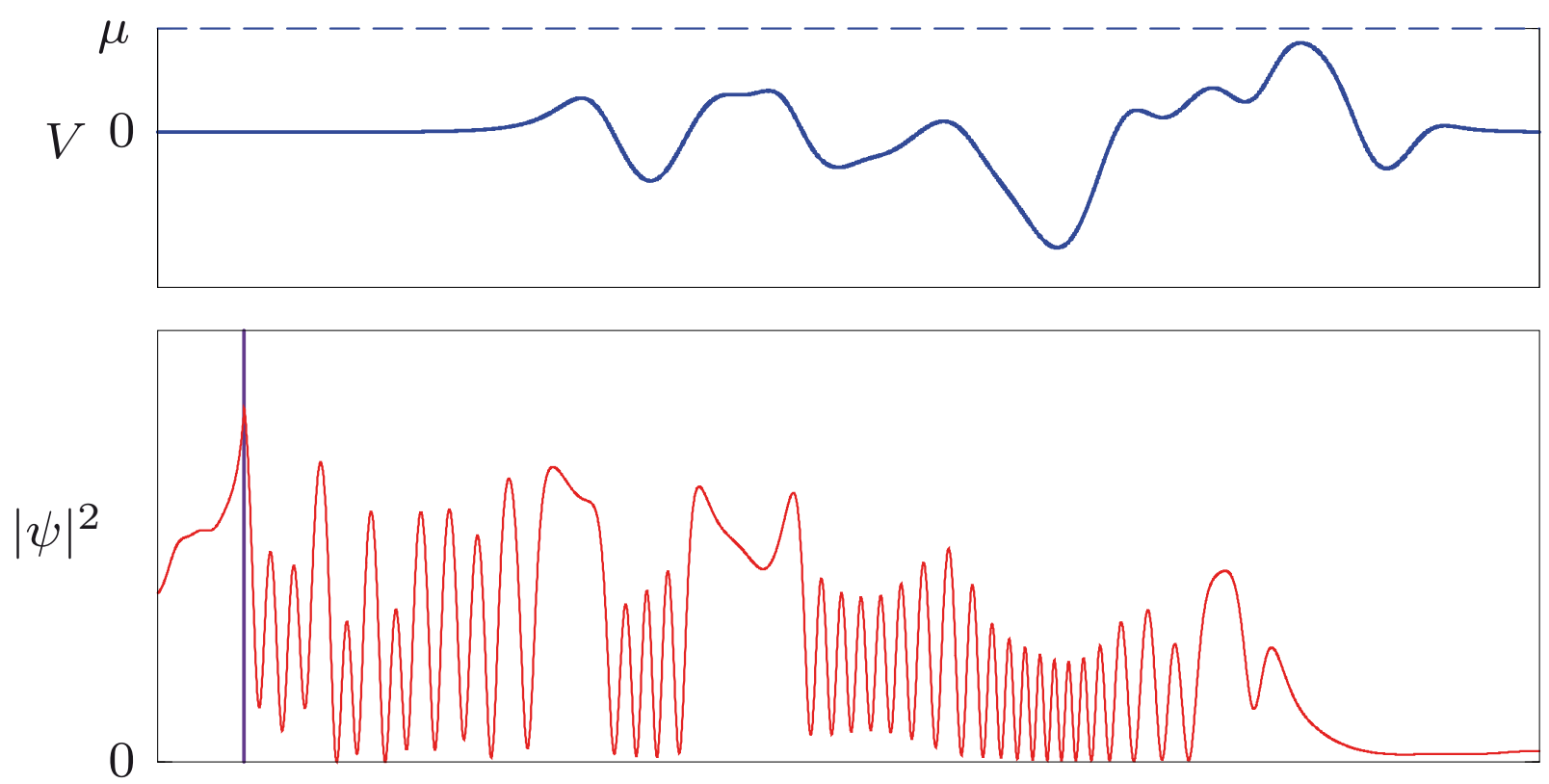


\section{Transport through longer scattering potentials}

Finite interaction between the atoms: $g|\psi|^{2} \simeq 0.1 \mu$
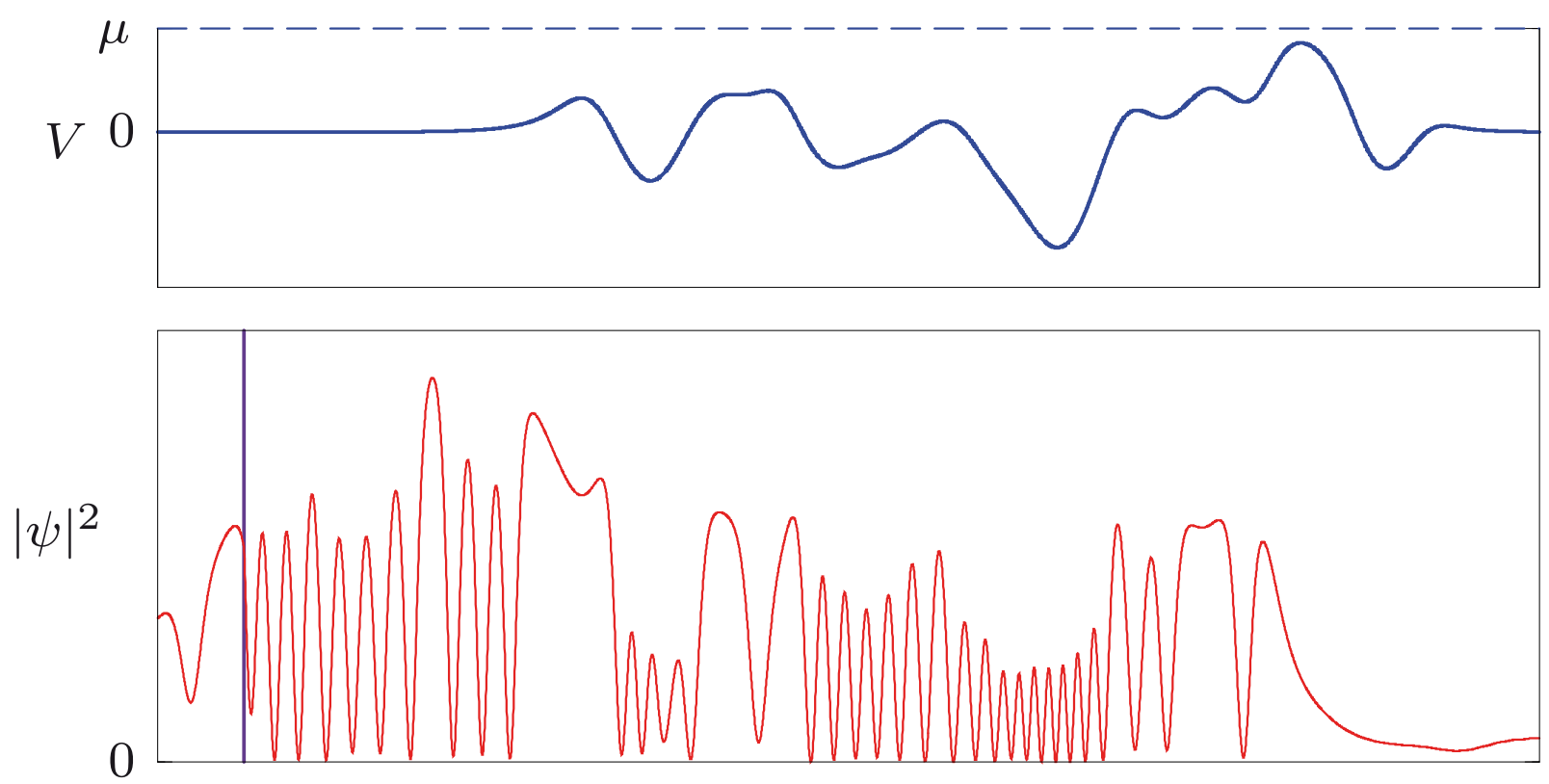


\section{Transport through longer scattering potentials}

Finite interaction between the atoms: $g|\psi|^{2} \simeq 0.1 \mu$
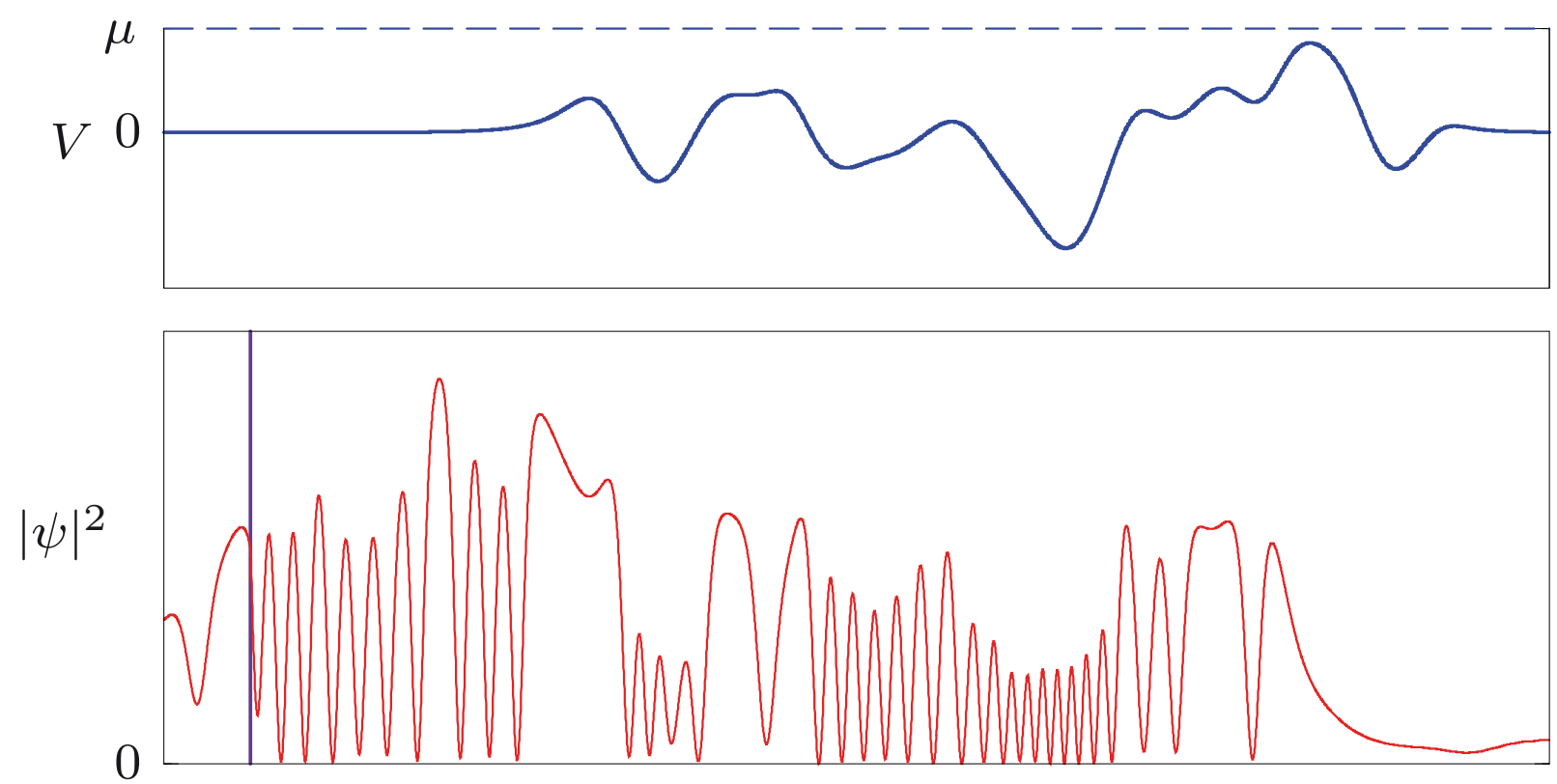

$x$

$\longrightarrow$ permanently time-dependent scattering

B. Spivak and A. Zyuzin, PRL 84, 1970 (2000)

S. E. Skipetrov and R. Maynard, PRL 85, 736 (2000) 



\section{Transport through longer scattering potentials}

Finite interaction between the atoms: $g|\psi|^{2} \simeq 0.1 \mu$
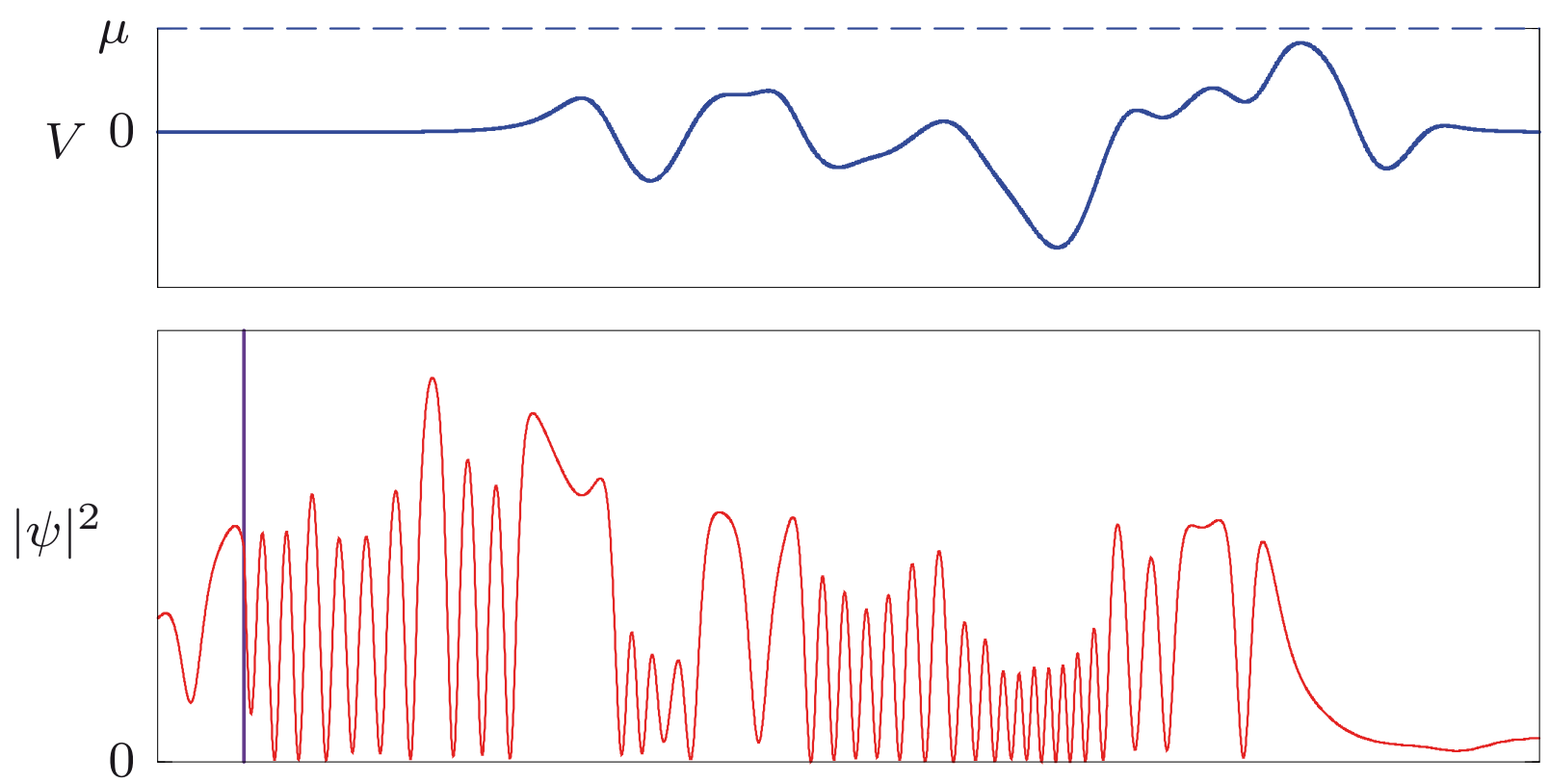

$x$

$\longrightarrow$ validity of the mean-field theory ?? 


\section{Beyond the Gross-Pitaevskii equation}

$$
\begin{aligned}
i \hbar \frac{\partial}{\partial t} \hat{\psi}(x, t)= & \left(-\frac{\hbar^{2}}{2 m} \frac{\partial^{2}}{\partial x^{2}}+V(x)+g \hat{\psi}^{\dagger}(x, t) \hat{\psi}(x, t)\right) \hat{\psi}(x, t) \\
& +S(x, t) \exp (-i \mu t / \hbar)
\end{aligned}
$$




\section{Beyond the Gross-Pitaevskii equation}

$$
\begin{aligned}
i \hbar \frac{\partial}{\partial t} \hat{\psi}(x, t)= & \left(-\frac{\hbar^{2}}{2 m} \frac{\partial^{2}}{\partial x^{2}}+V(x)+g \hat{\psi}^{\dagger}(x, t) \hat{\psi}(x, t)\right) \hat{\psi}(x, t) \\
& +S(x, t) \exp (-i \mu t / \hbar)
\end{aligned}
$$

Bogoliubov ansatz: $\hat{\psi}(x, t)=\langle\hat{\psi}(x, t)\rangle+\delta \hat{\psi}(x, t)$

$\longrightarrow$ derive coupled equations for the expectation values

$$
\begin{aligned}
& \langle\hat{\psi}(x, t)\rangle,\left\langle\delta \hat{\psi}\left(x_{1}, t\right) \delta \hat{\psi}\left(x_{2}, t\right)\right\rangle,\left\langle\delta \hat{\psi}^{\dagger}\left(x_{1}, t\right) \delta \hat{\psi}\left(x_{2}, t\right)\right\rangle, \\
& \left\langle\delta \hat{\psi}\left(x_{1}, t\right) \delta \hat{\psi}\left(x_{2}, t\right) \delta \hat{\psi}\left(x_{3}, t\right)\right\rangle, \ldots
\end{aligned}
$$

$\longrightarrow$ Hartree-Fock-Bogoliubov theory 


\section{Beyond the Gross-Pitaevskii equation}

$$
\begin{aligned}
i \hbar \frac{\partial}{\partial t} \hat{\psi}(x, t)= & \left(-\frac{\hbar^{2}}{2 m} \frac{\partial^{2}}{\partial x^{2}}+V(x)+g \hat{\psi}^{\dagger}(x, t) \hat{\psi}(x, t)\right) \hat{\psi}(x, t) \\
& +S(x, t) \exp (-i \mu t / \hbar)
\end{aligned}
$$

Mean-field regime:

- large density $n \propto|S|^{2}$ of atoms emitted by the source

- small 1D interaction strength $g \propto a_{s}$

- finite mean-field interaction term $\propto n a_{s}$

$\Longrightarrow$ well-defined truncation scheme in the formal limit $n \rightarrow \infty, a_{s} \rightarrow 0, n a_{s} \sim$ const:

take into account terms of order $n^{1 / 2}, n^{0}, n^{-1 / 2}$, and $n^{-1}$ 


\section{Beyond the Gross-Pitaevskii equation}

$$
\begin{array}{rlr}
\Psi(x, t) \equiv\langle\hat{\psi}(x, t)\rangle & \quad \Gamma(x, y, t) \equiv\left\langle\delta \hat{\psi}^{\dagger}(y, t) \delta \hat{\psi}(x, t)\right\rangle, \quad \Phi(x, y, t) \equiv\langle\delta \hat{\psi}(y, t) \delta \hat{\psi}(x, t)\rangle \\
i \hbar \frac{\partial}{\partial t} \Psi(x, t)= & \mathrm{H}_{0}^{(x)} \Psi(x, t)+g|\Psi(x, t)|^{2} \Psi(x, t)+S(x, t) \exp (-i \mu t / \hbar) & \\
& +2 g \Psi(x, t) \Gamma(x, x, t)+g \Psi^{\dagger}(x, t) \Phi(x, x, t) & \\
i \hbar \frac{\partial}{\partial t} \Phi(x, y, t)=\quad & \left.\mathrm{H}_{0}^{(x)}+2 g|\Psi(x, t)|^{2}+\mathrm{H}_{0}^{(y)}+2 g|\Psi(y, t)|^{2}\right] \Phi(x, y, t) & \\
& +g \delta(x-y) \Psi(x, t) \Psi(y, t) & \\
& +g \Psi^{2}(y, t) \Gamma(x, y, t)+g \Psi^{2}(x, t) \Gamma(y, x, t) & \\
& +g \delta(x-y) \Phi(x, y, t)+2 g[\Gamma(x, x, t)+\Gamma(y, y, t)] \Phi(x, y, t) \\
& +g \Gamma(y, x, t) \Phi(x, x, t)+g \Gamma(x, y, t) \Phi(y, y, t) & \\
& {\left[\mathrm{H}_{0}^{(x)}+2 g|\Psi(x, t)|^{2}-\mathrm{H}_{0}^{(y)}-2 g|\Psi(y, t)|^{2}\right] \Gamma(x, y, t)} \\
& +g \Psi^{2}(x, t) \Phi^{\star}(x, y, t)-g\left(\Psi^{\star}(y, t)\right)^{2} \Phi(x, y, t) & \\
& +2 g\left[\Gamma(x, x, t)-\Gamma^{\star}(y, y, t)\right] \Gamma(x, y, t) & \\
& +g \Phi^{\star}(x, y, t) \Phi(x, x, t)-g \Phi(x, y, t) \Phi^{\star}(y, y, t)
\end{array}
$$




\section{Depletion in extended potentials}

Quasi-stationary scattering on the Gross-Pitaevskii level:

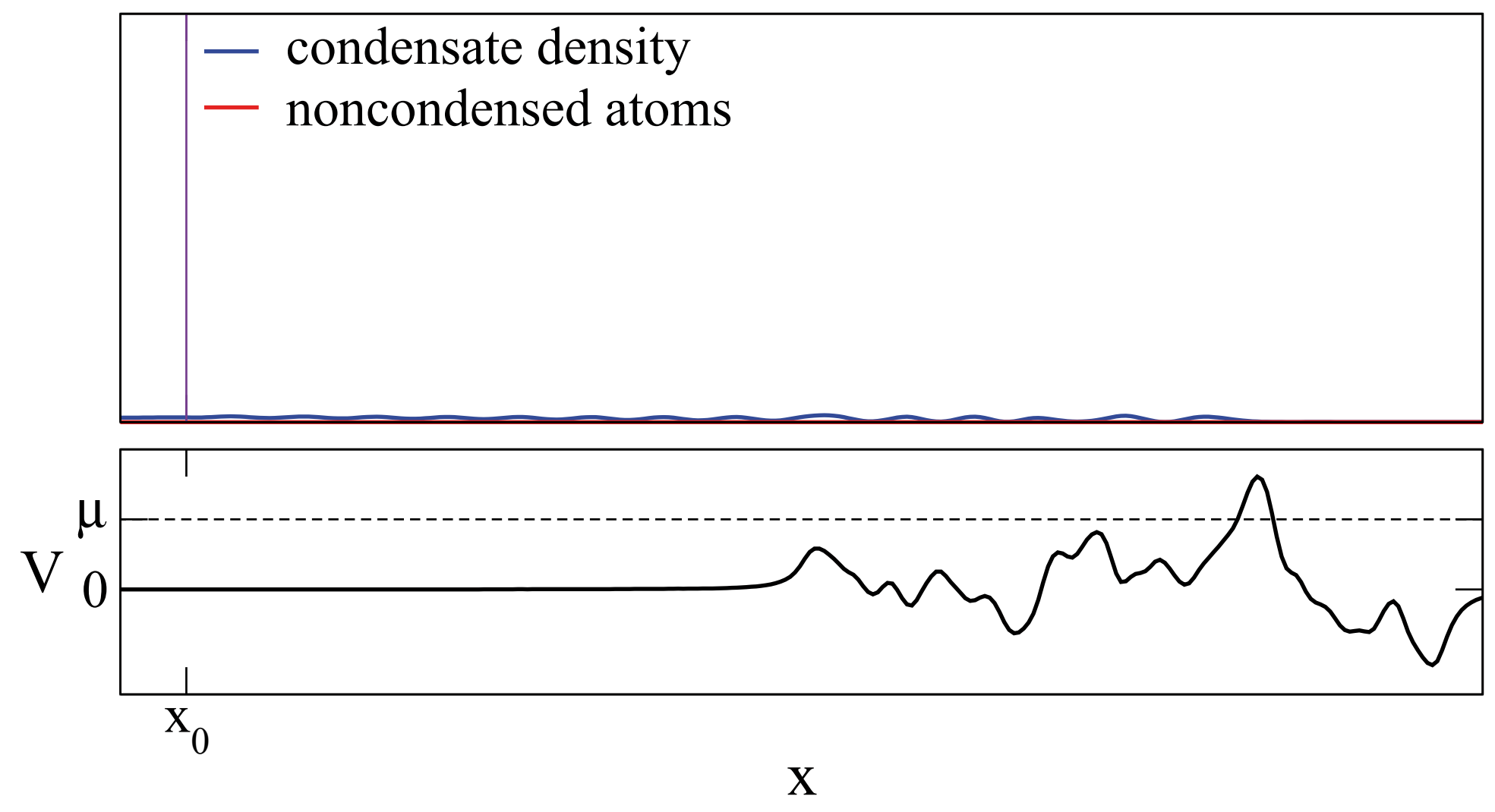




\section{Depletion in extended potentials}

Quasi-stationary scattering on the Gross-Pitaevskii level:

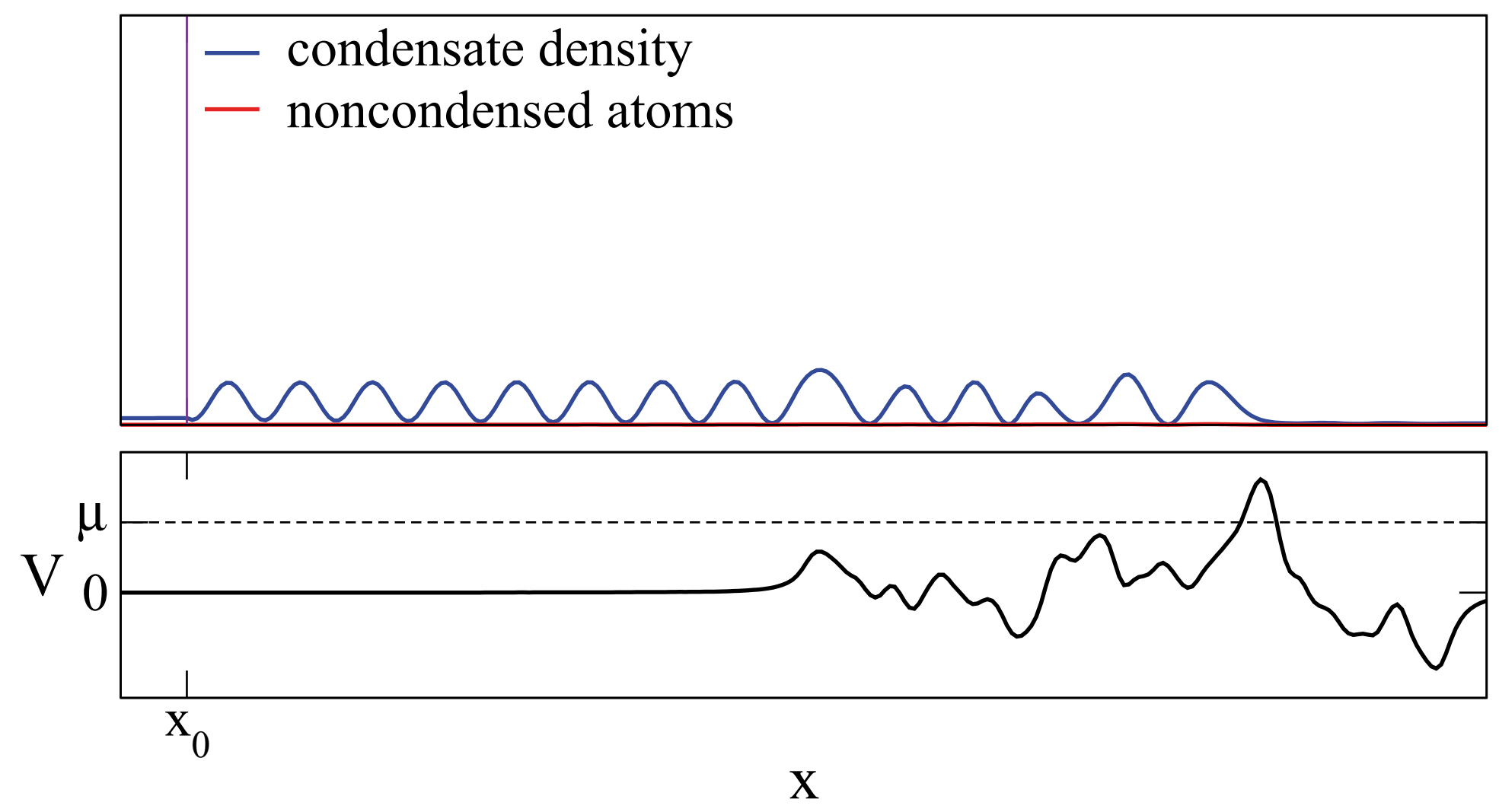




\section{Depletion in extended potentials}

Quasi-stationary scattering on the Gross-Pitaevskii level:

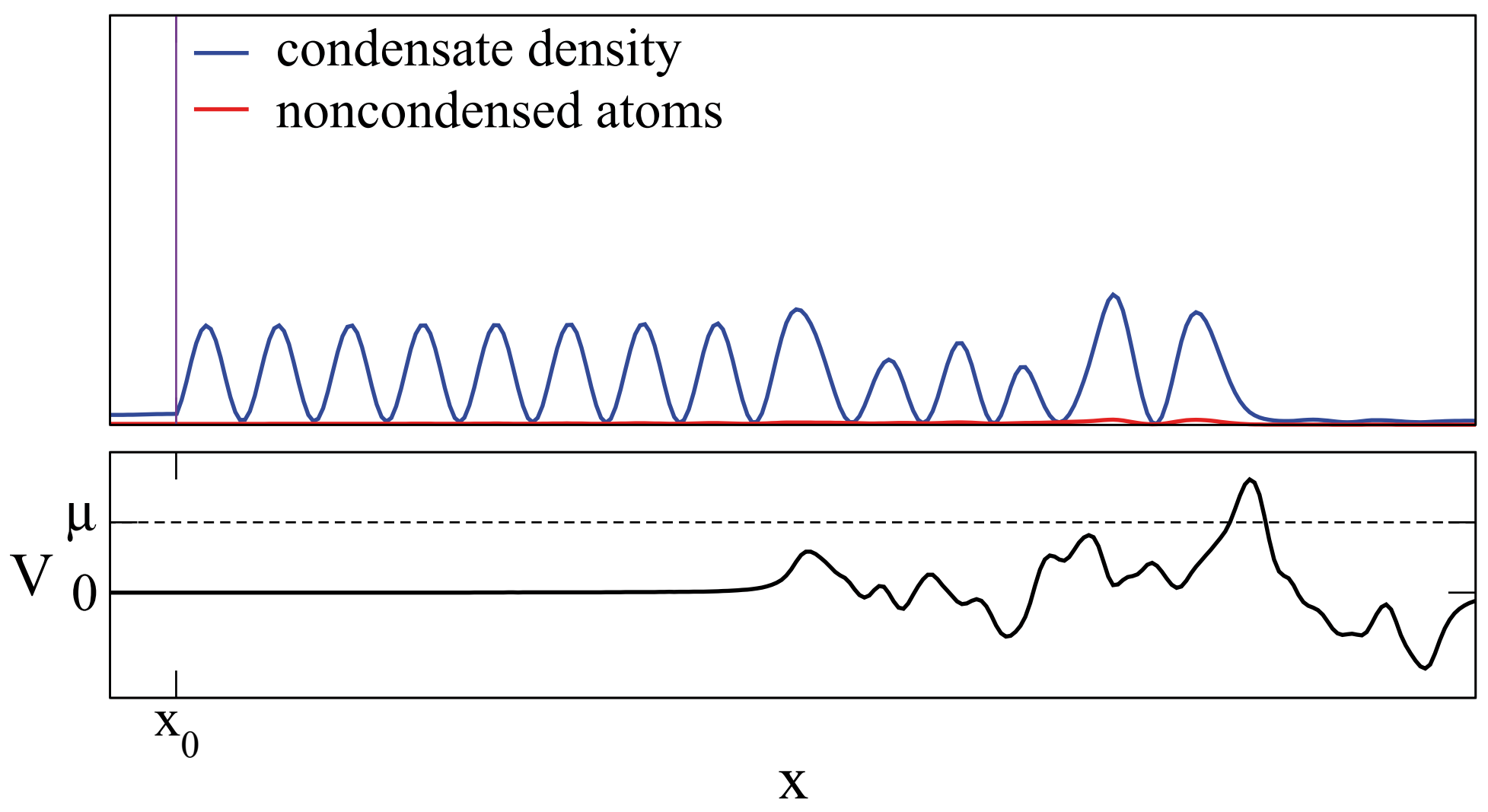




\section{Depletion in extended potentials}

Quasi-stationary scattering on the Gross-Pitaevskii level:

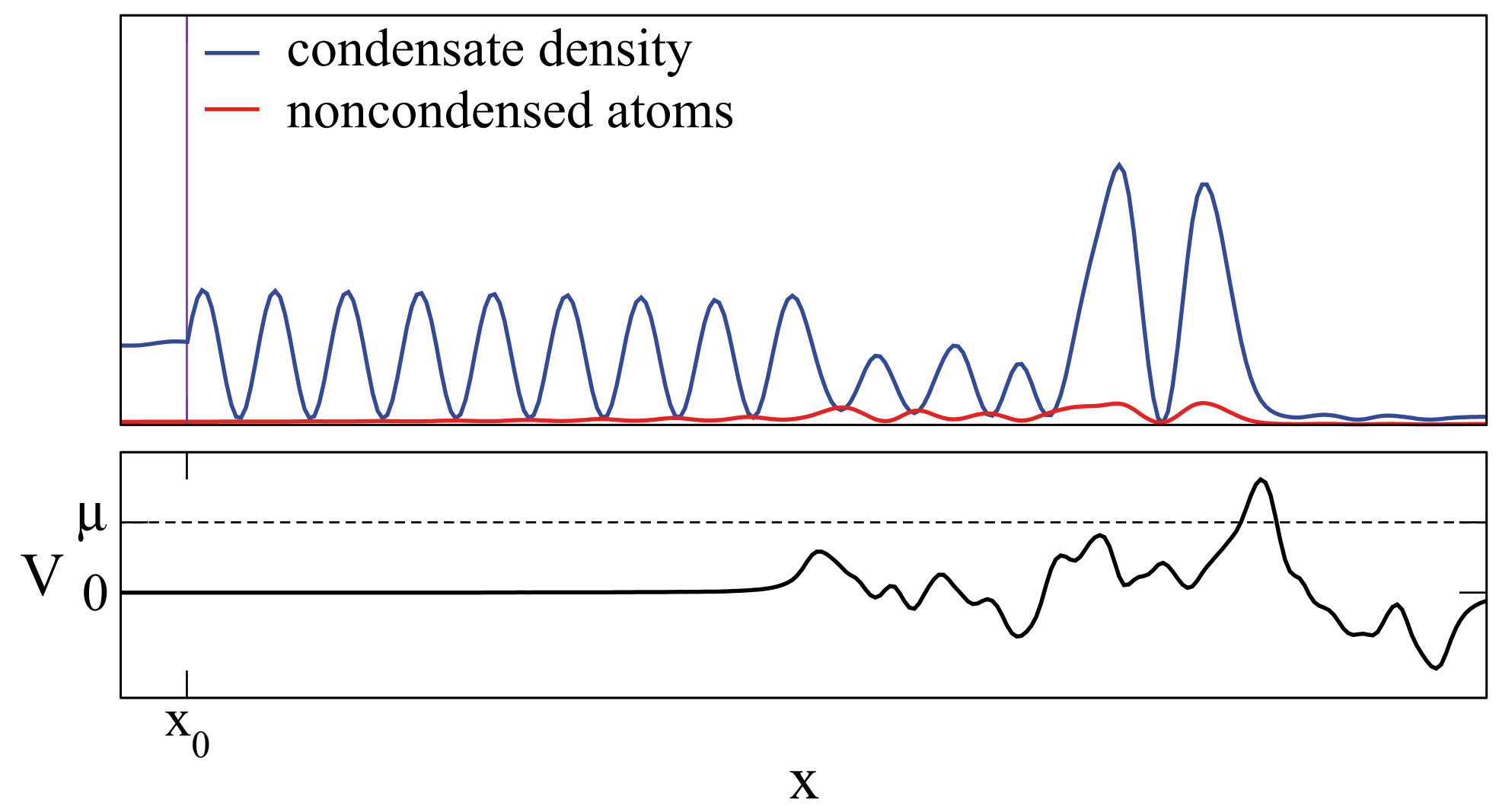




\section{Depletion in extended potentials}

Quasi-stationary scattering on the Gross-Pitaevskii level:
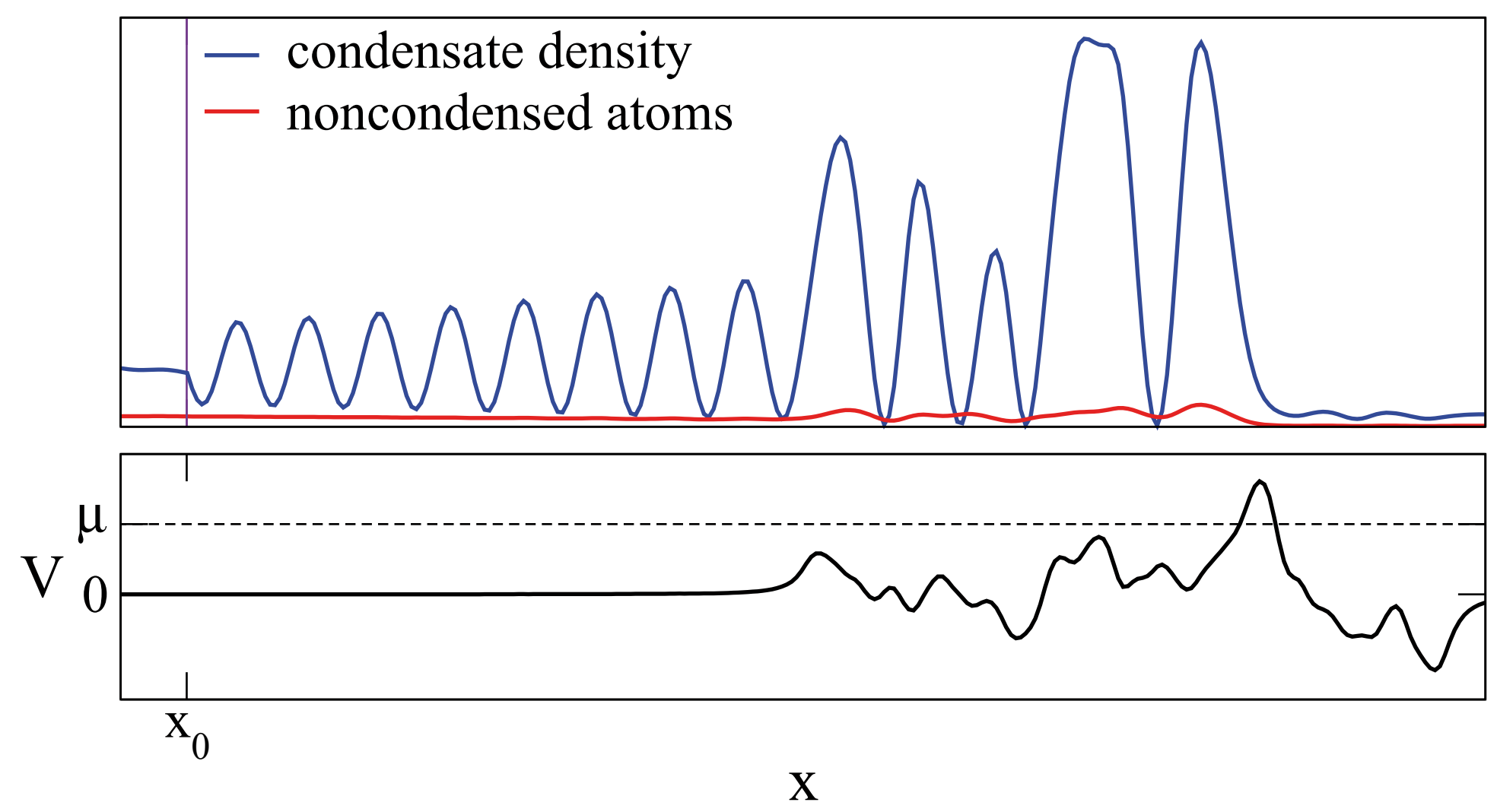


\section{Depletion in extended potentials}

Quasi-stationary scattering on the Gross-Pitaevskii level:

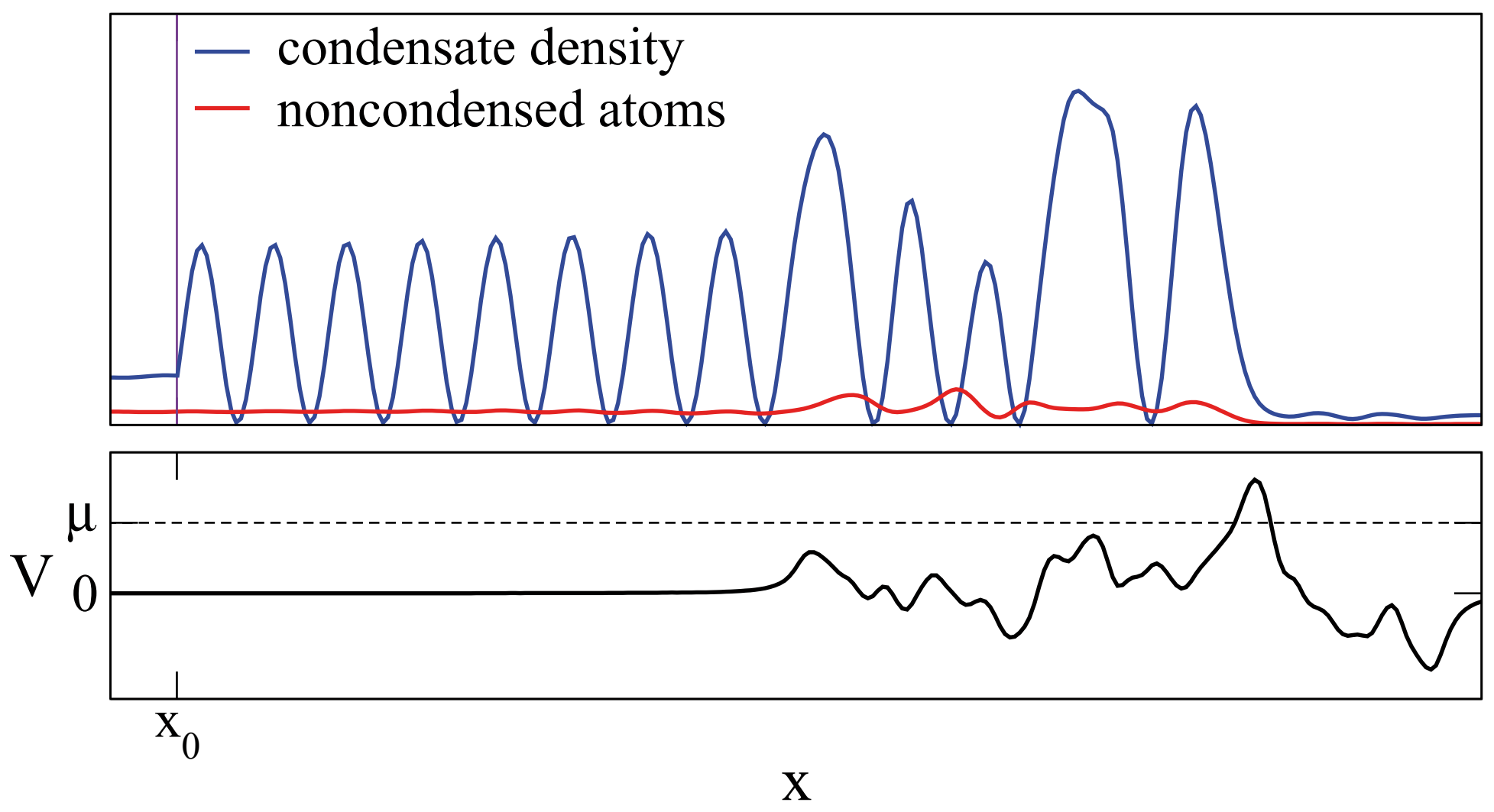




\section{Depletion in extended potentials}

Quasi-stationary scattering on the Gross-Pitaevskii level:

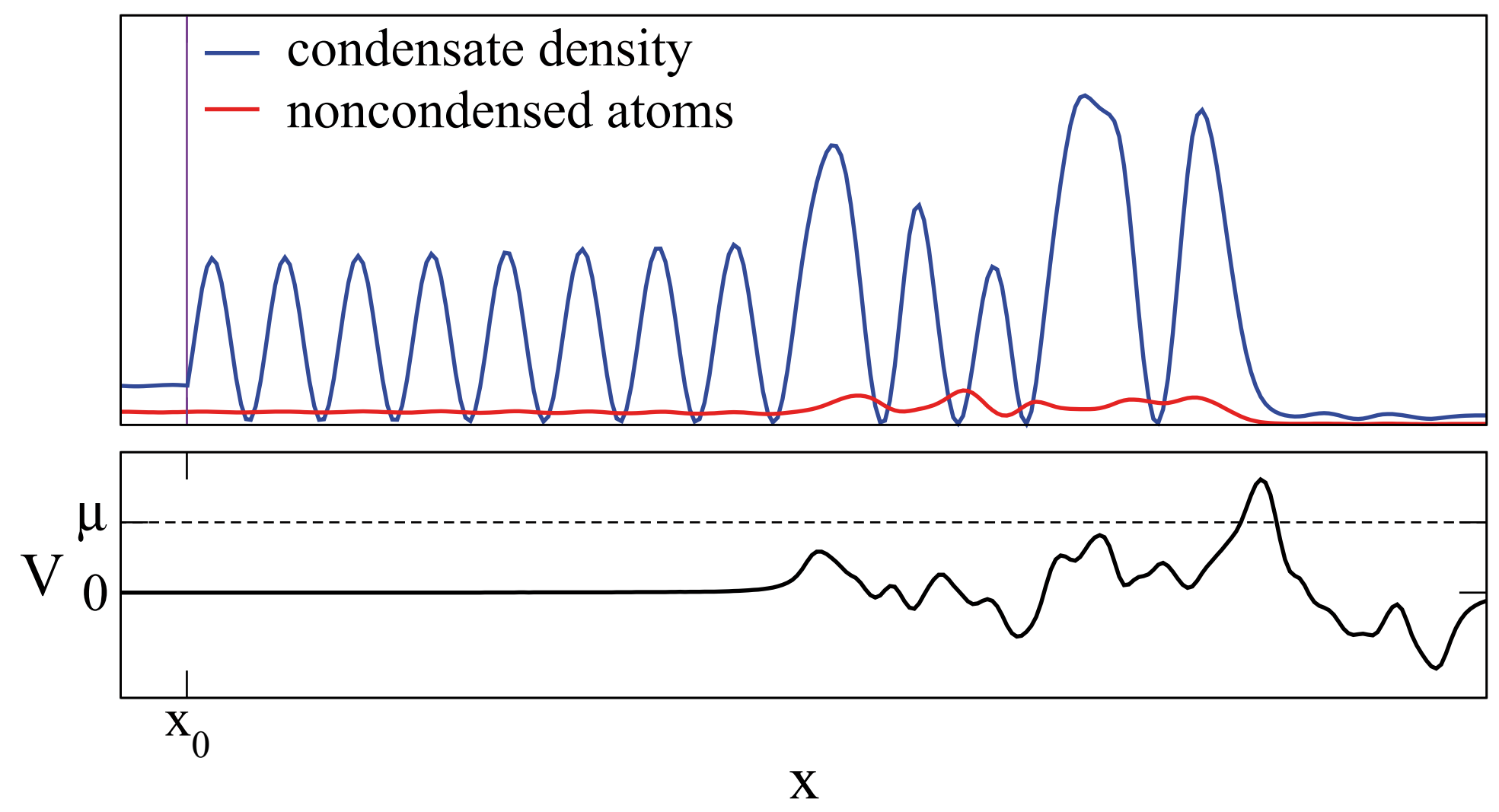




\section{Depletion in extended potentials}

Quasi-stationary scattering on the Gross-Pitaevskii level:

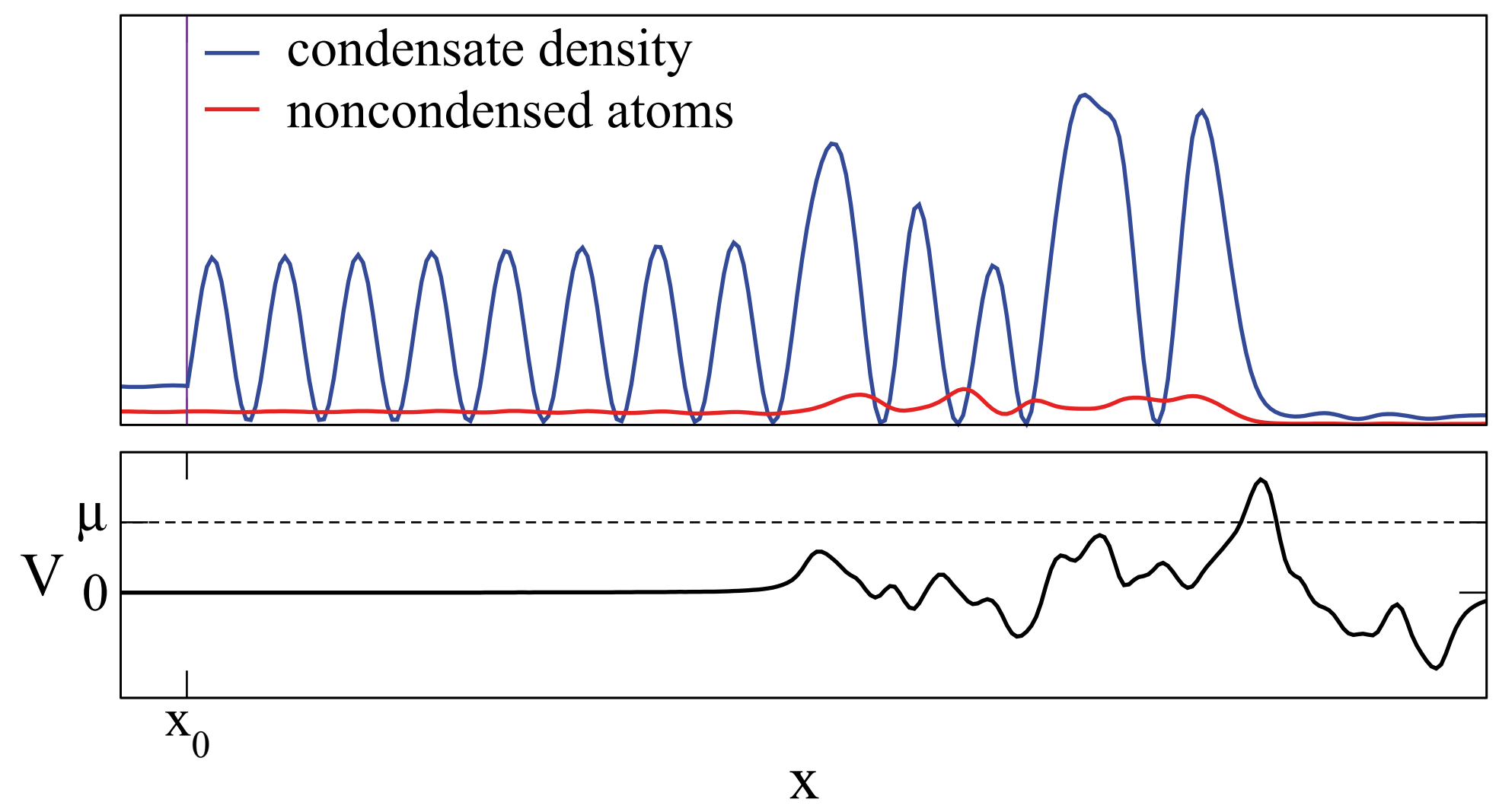




\section{Depletion in extended potentials}

Quasi-stationary scattering on the Gross-Pitaevskii level:

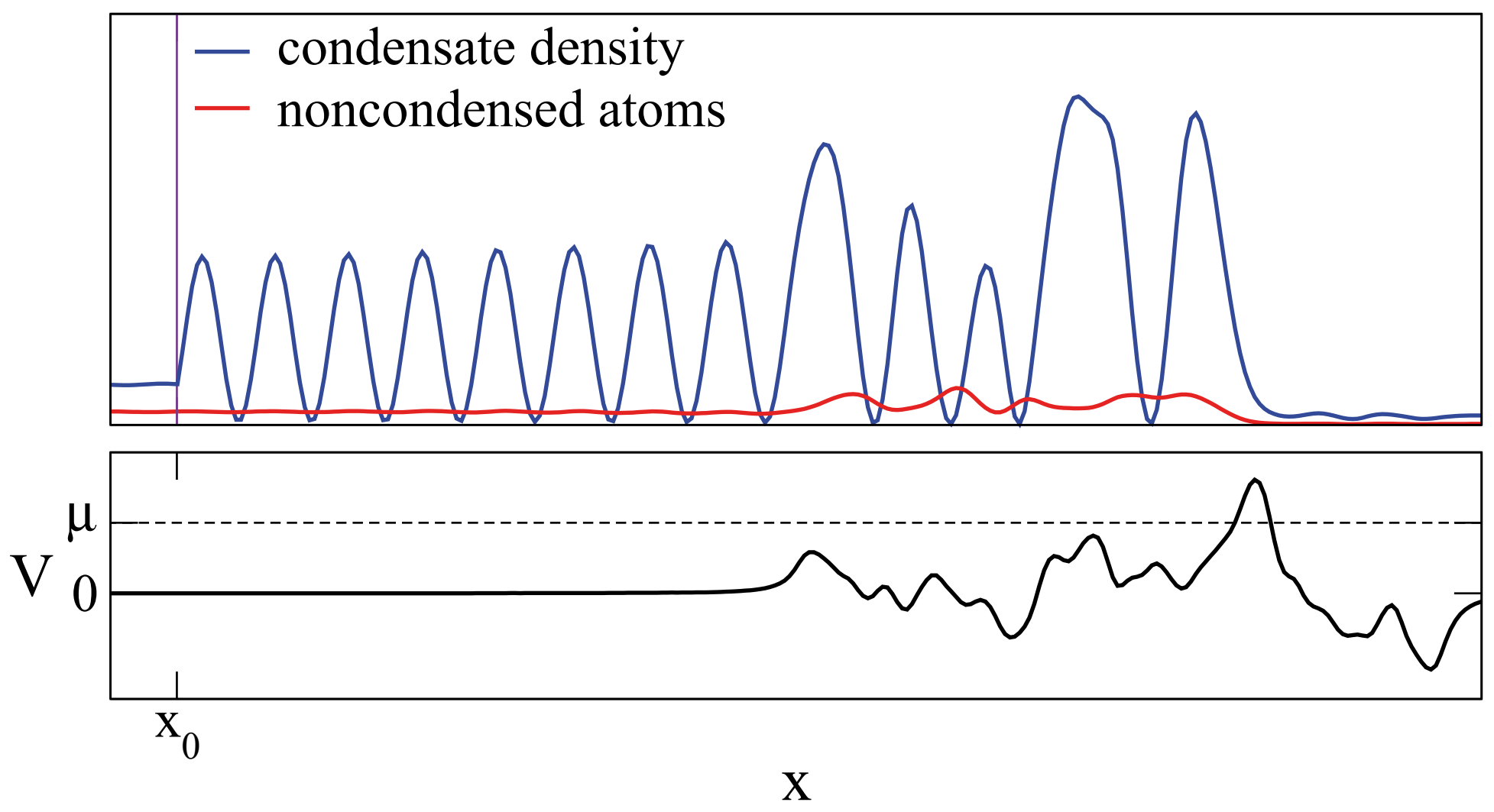




\section{Depletion in extended potentials}

Quasi-stationary scattering on the Gross-Pitaevskii level:

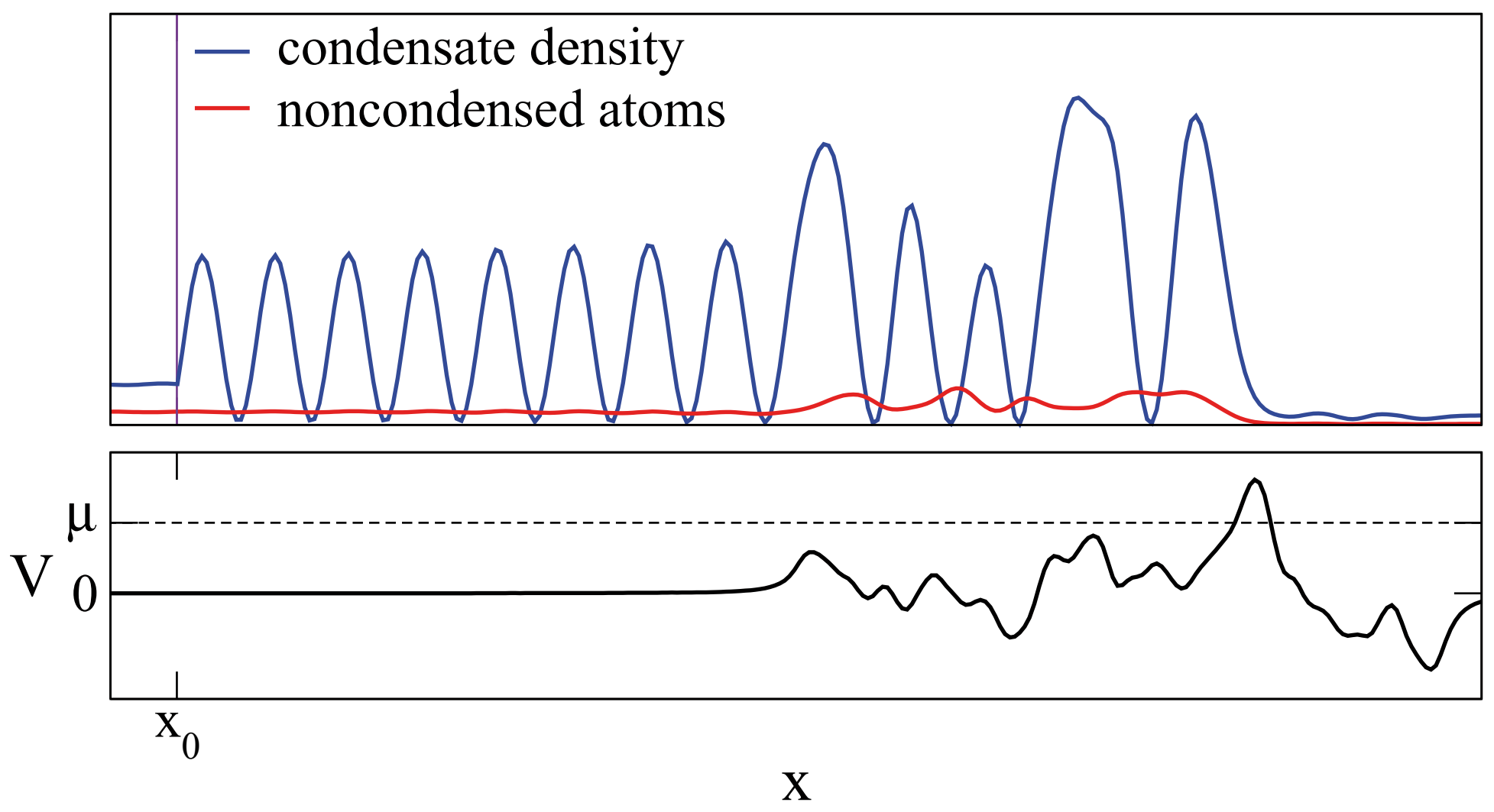




\section{Depletion in extended potentials}

Quasi-stationary scattering on the Gross-Pitaevskii level:

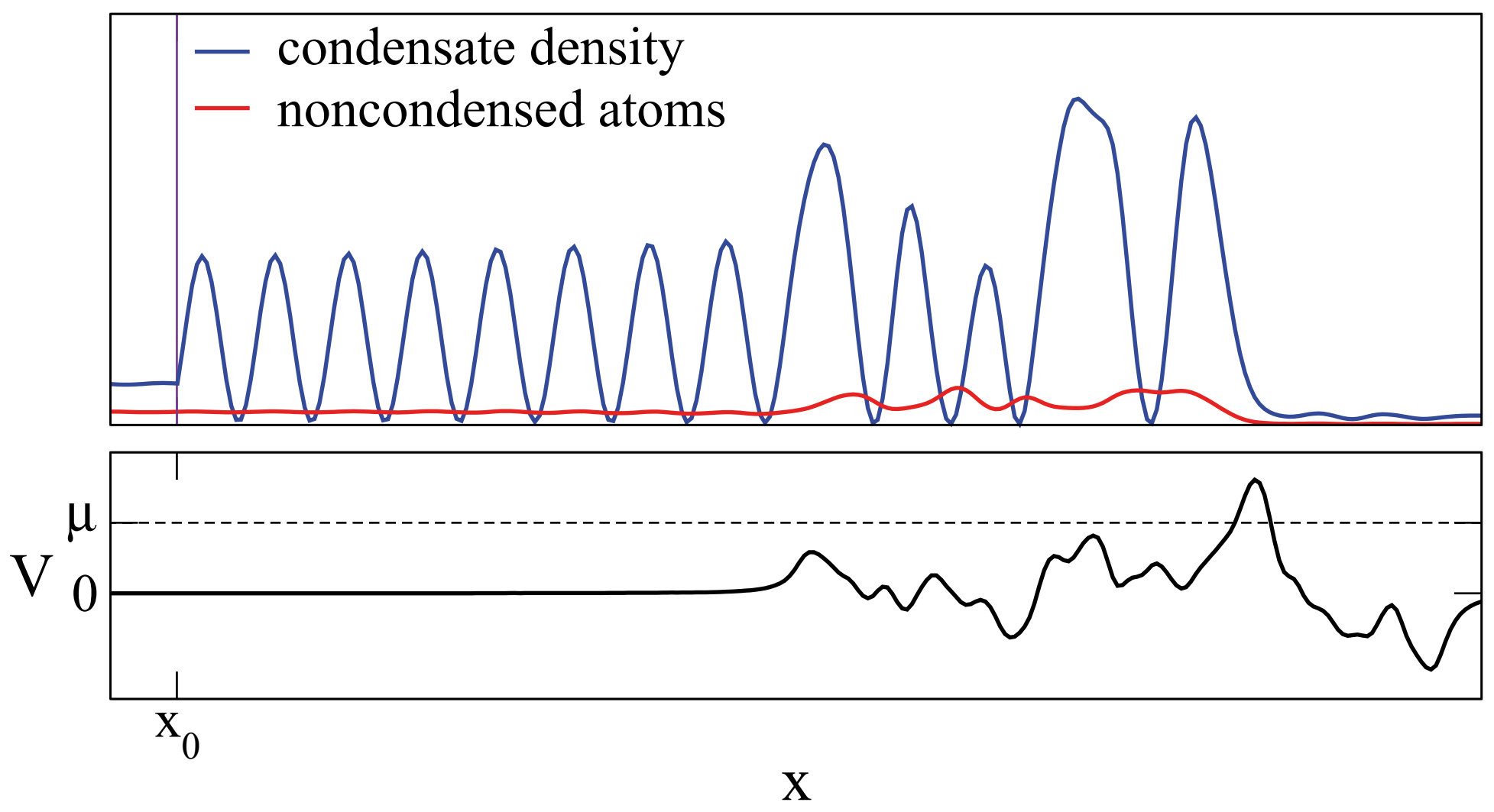




\section{Depletion in extended potentials}

Quasi-stationary scattering on the Gross-Pitaevskii level:

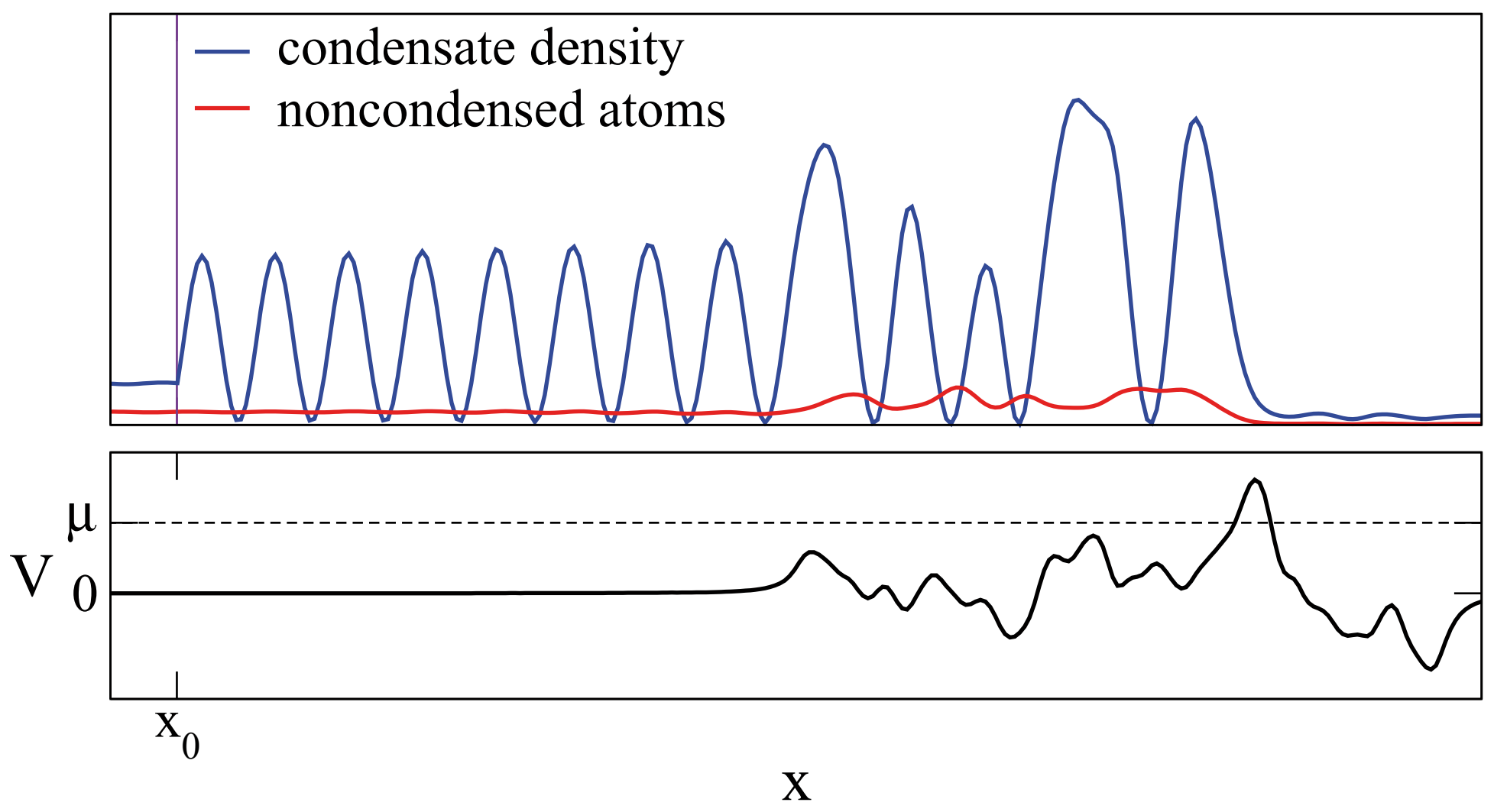




\section{Depletion in extended potentials}

Quasi-stationary scattering on the Gross-Pitaevskii level:

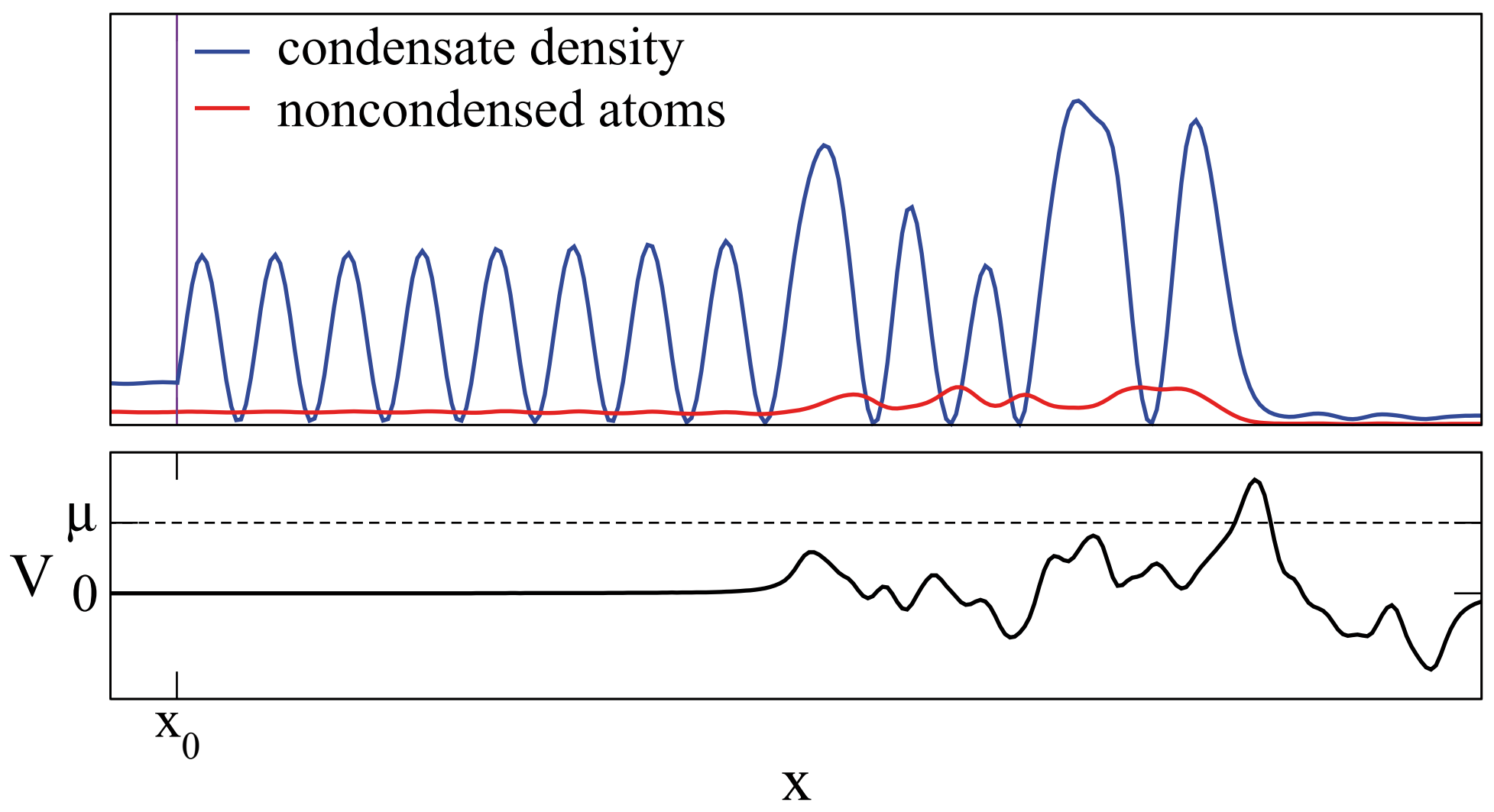




\section{Depletion in extended potentials}

Quasi-stationary scattering on the Gross-Pitaevskii level:

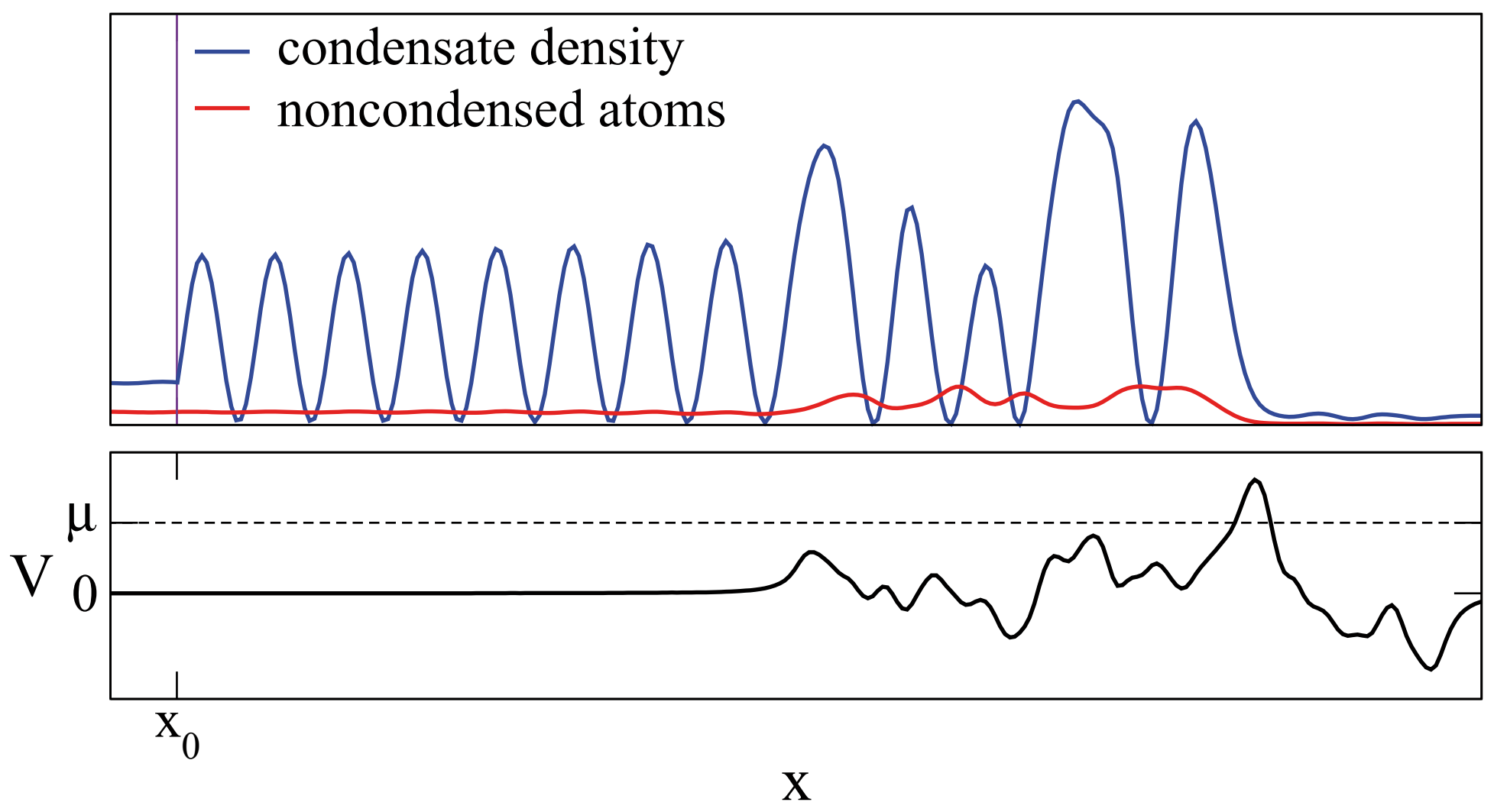




\section{Depletion in extended potentials}

Quasi-stationary scattering on the Gross-Pitaevskii level:

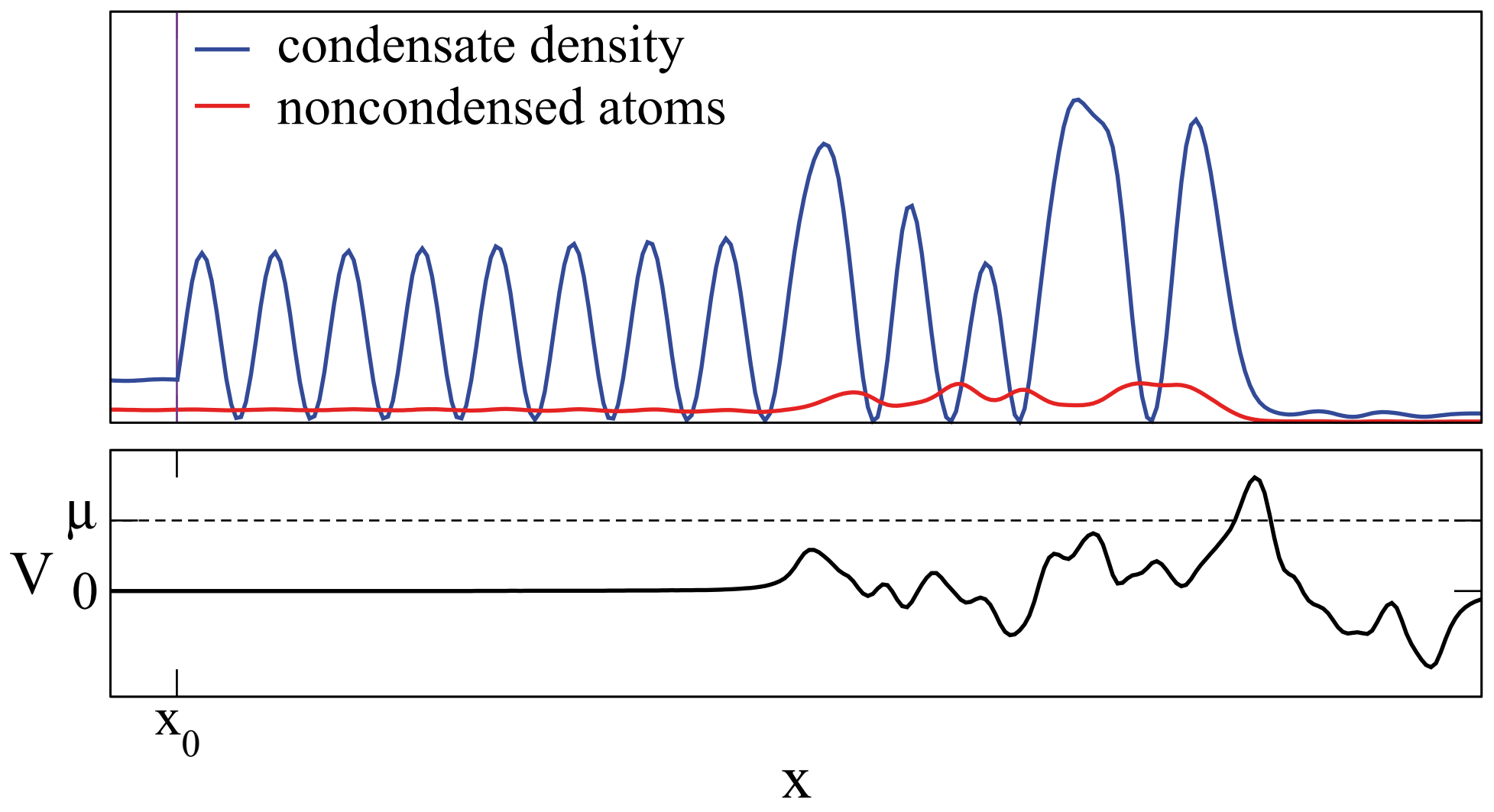




\section{Depletion in extended potentials}

Quasi-stationary scattering on the Gross-Pitaevskii level:

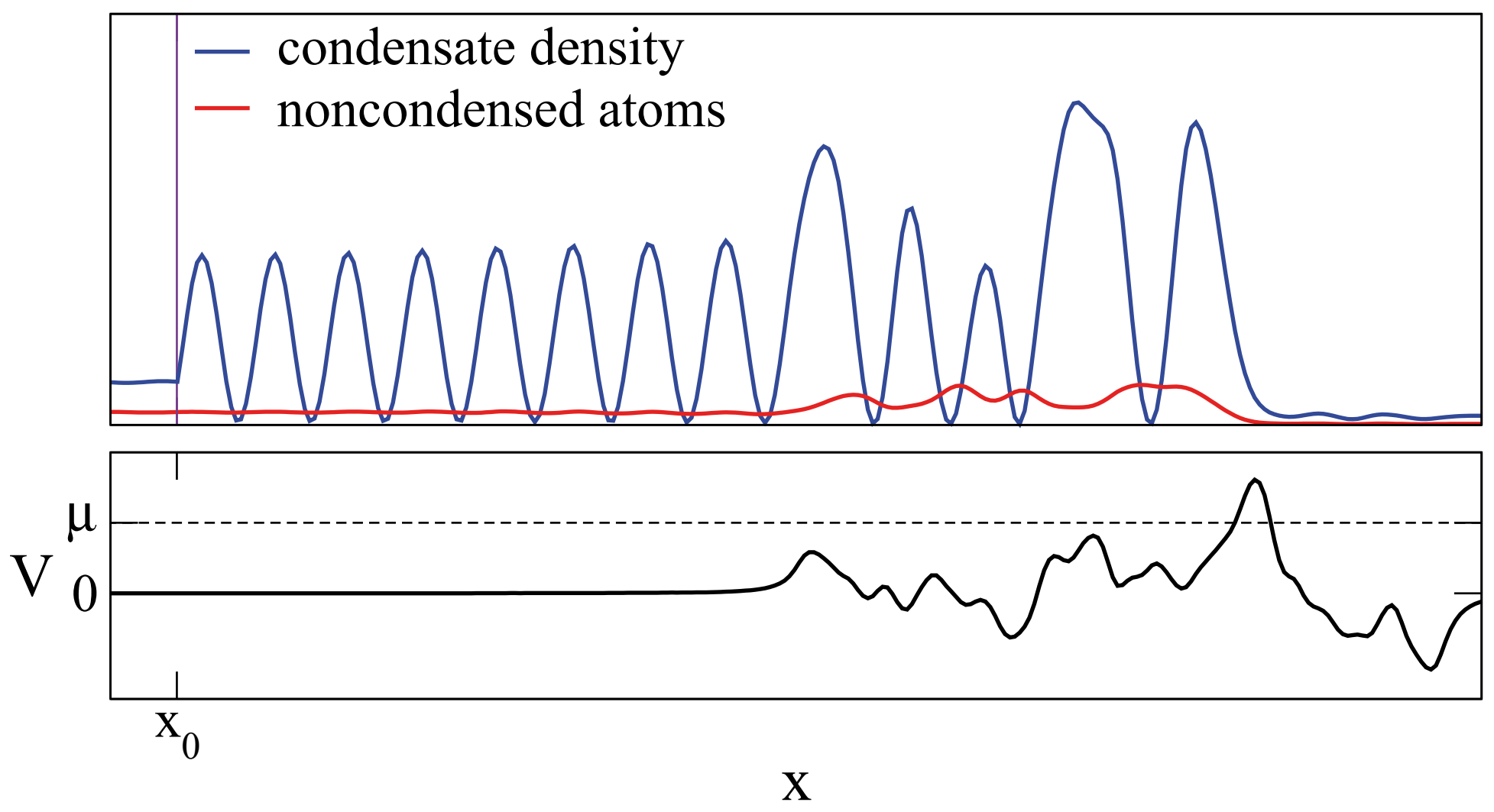




\section{Depletion in extended potentials}

Quasi-stationary scattering on the Gross-Pitaevskii level:

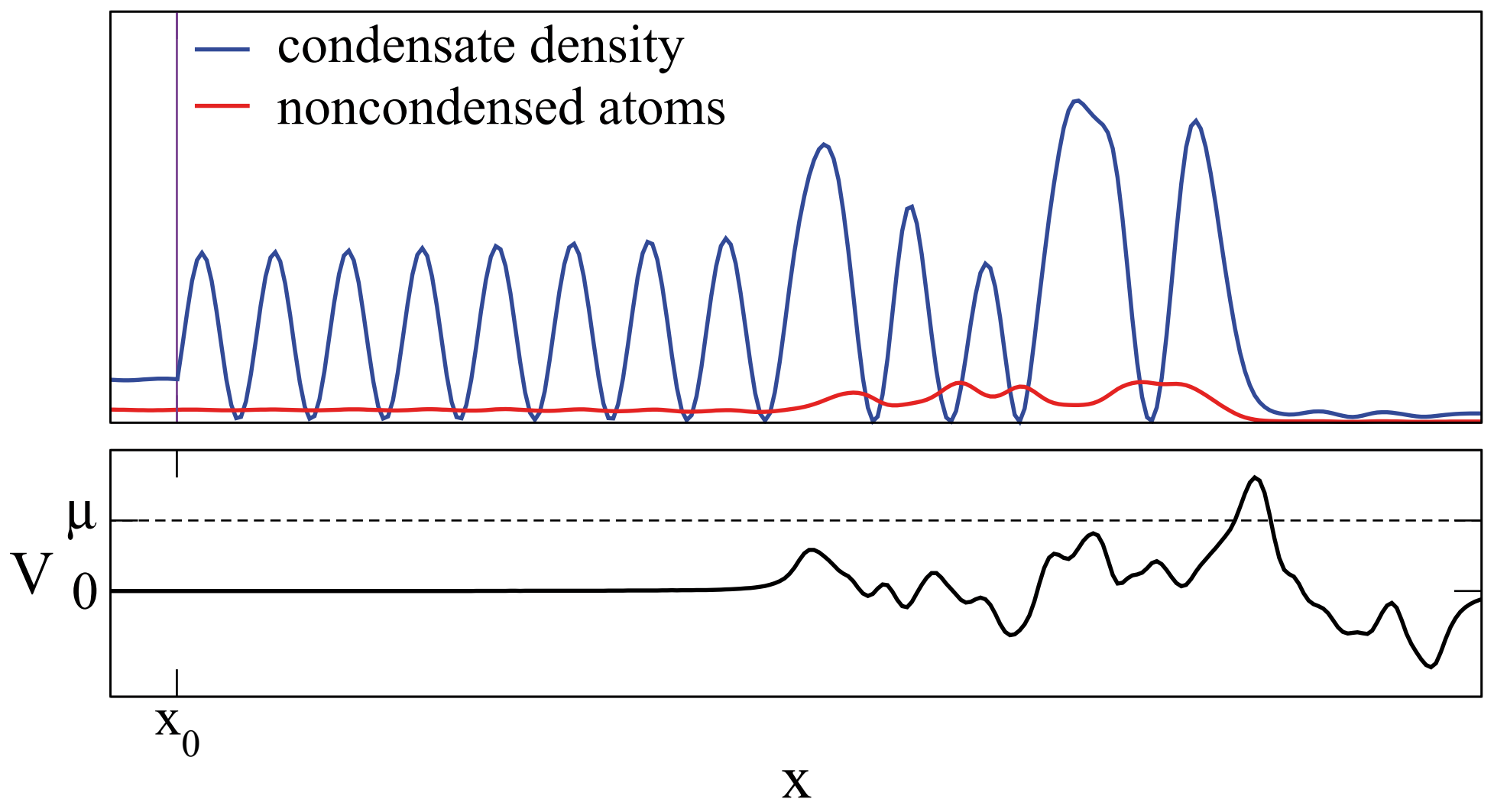




\section{Depletion in extended potentials}

Quasi-stationary scattering on the Gross-Pitaevskii level:

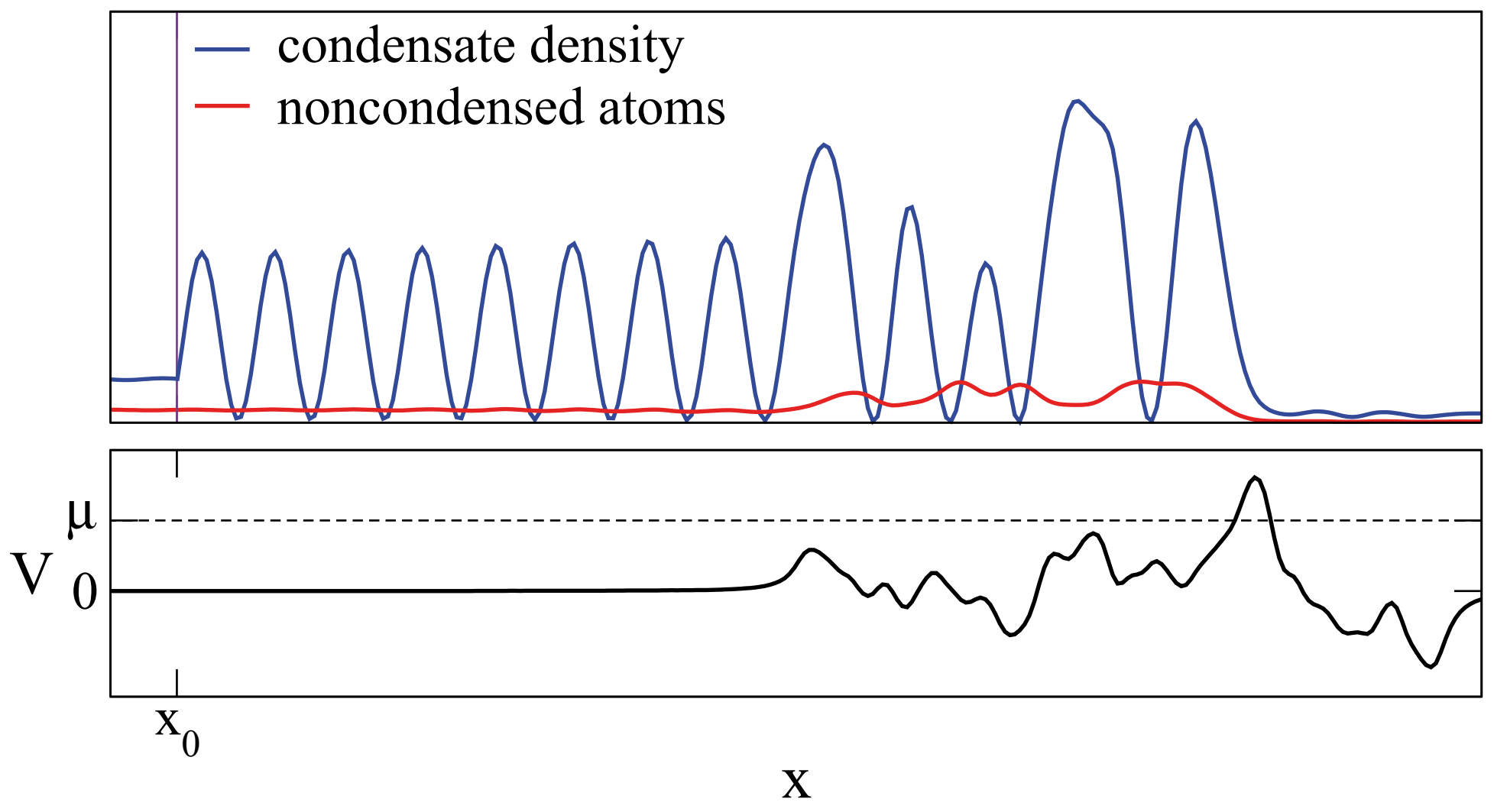




\section{Depletion in extended potentials}

Quasi-stationary scattering on the Gross-Pitaevskii level:

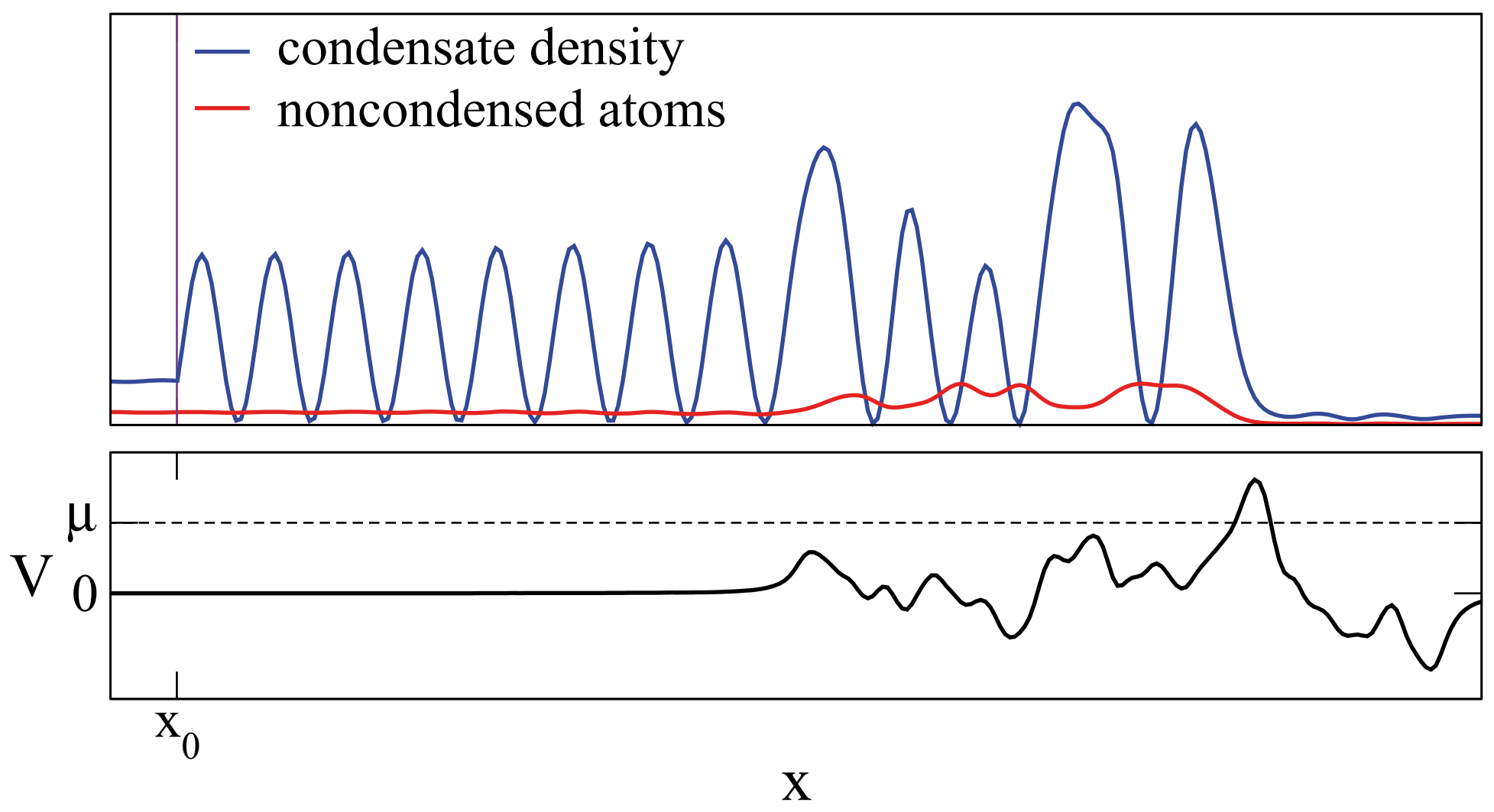




\section{Depletion in extended potentials}

Quasi-stationary scattering on the Gross-Pitaevskii level:

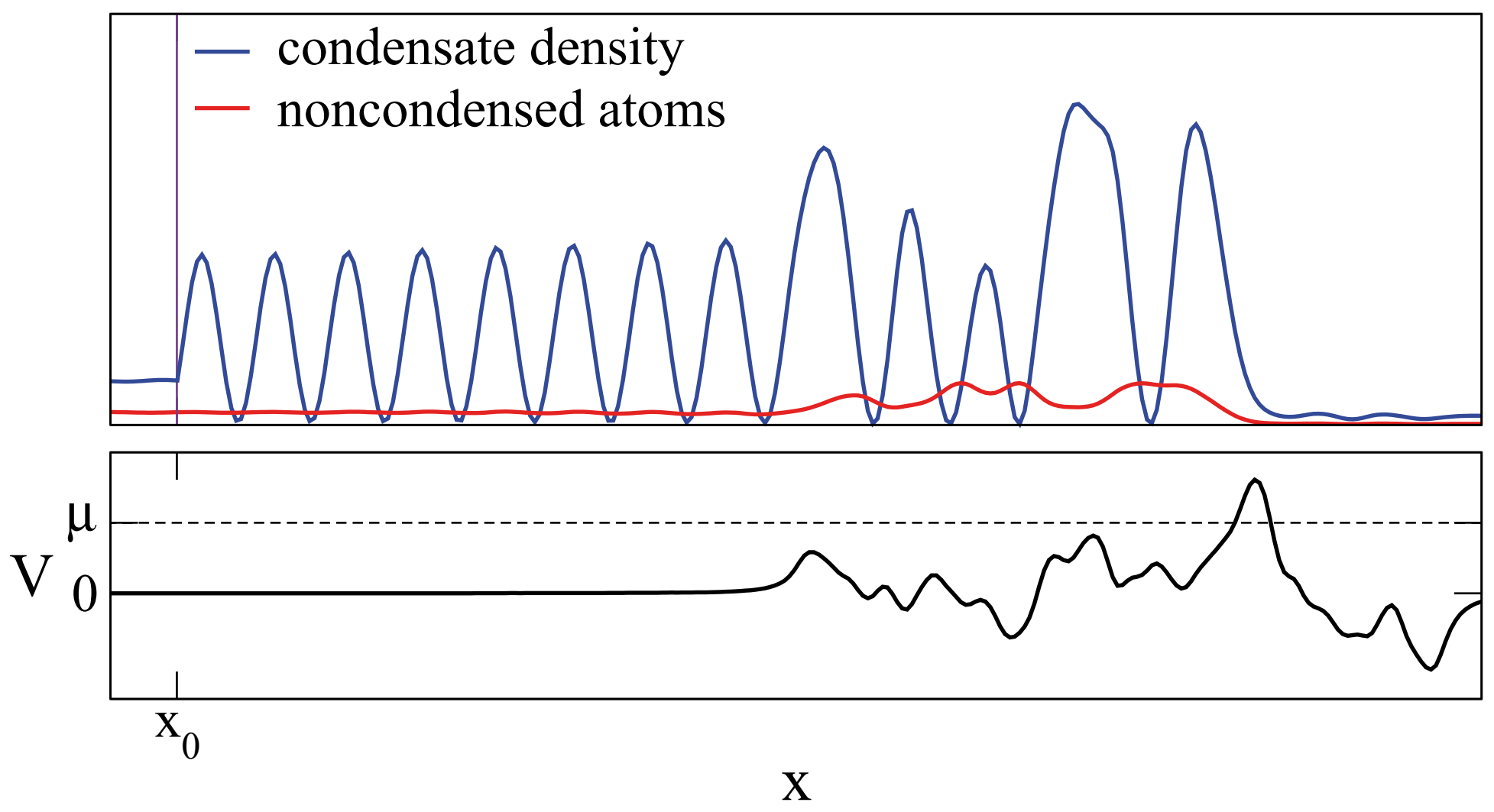




\section{Depletion in extended potentials}

Quasi-stationary scattering on the Gross-Pitaevskii level:
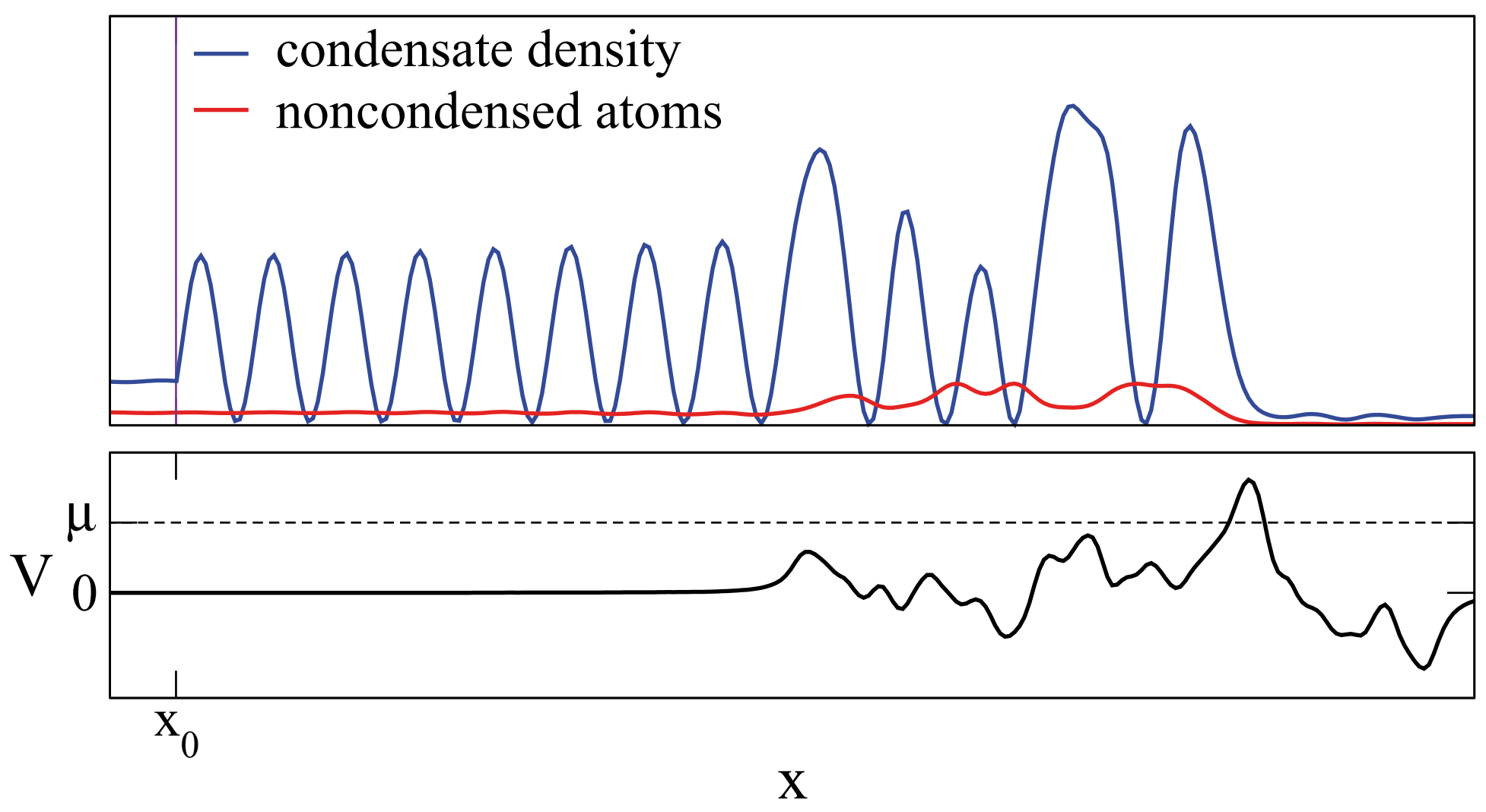

$\longrightarrow$ low depletion, coherent (mean-field-like) transport 


\section{Depletion in extended potentials}

Time-dependent scattering on the Gross-Pitaevskii level:

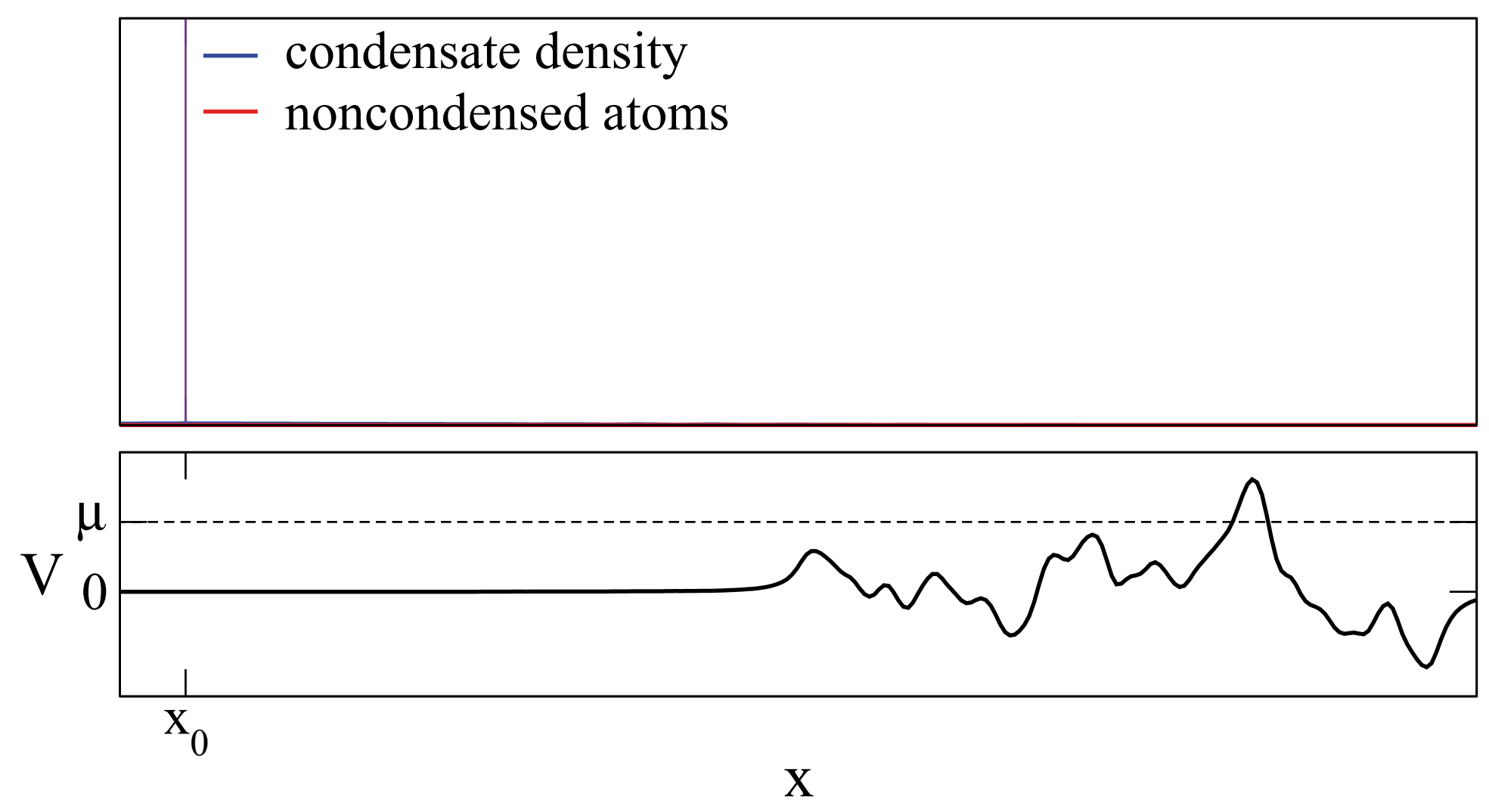




\section{Depletion in extended potentials}

Time-dependent scattering on the Gross-Pitaevskii level:

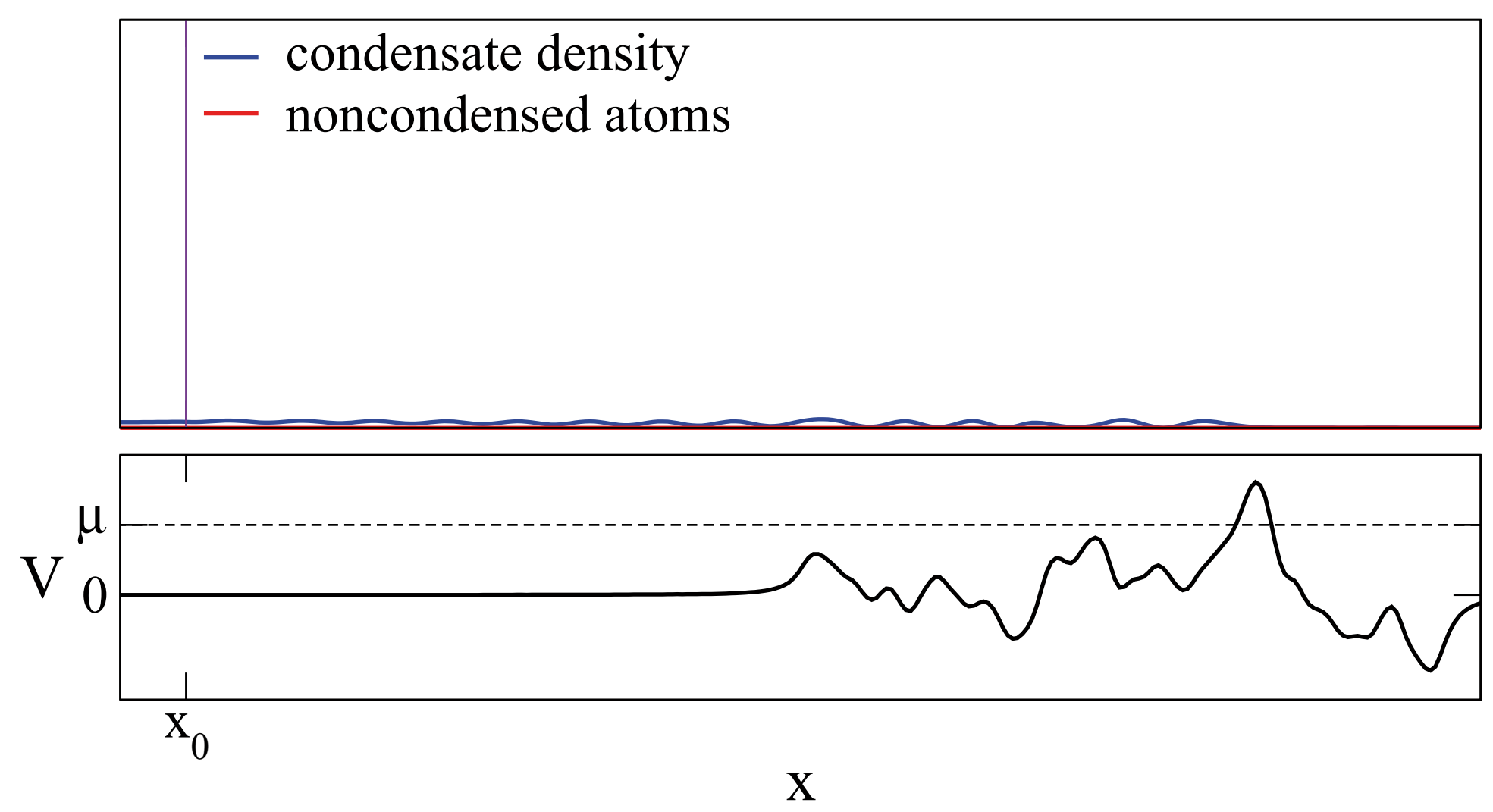




\section{Depletion in extended potentials}

Time-dependent scattering on the Gross-Pitaevskii level:

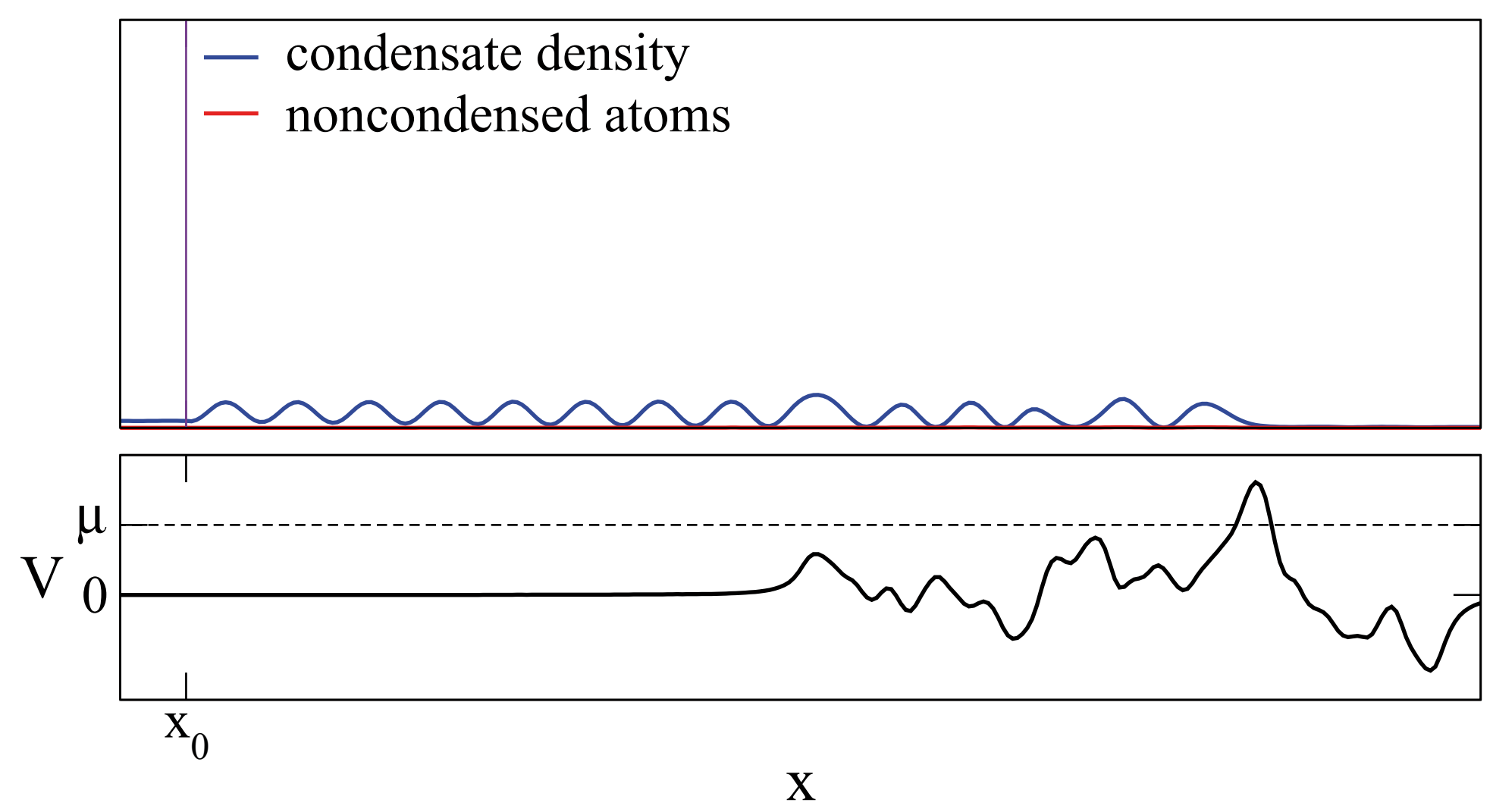




\section{Depletion in extended potentials}

Time-dependent scattering on the Gross-Pitaevskii level:

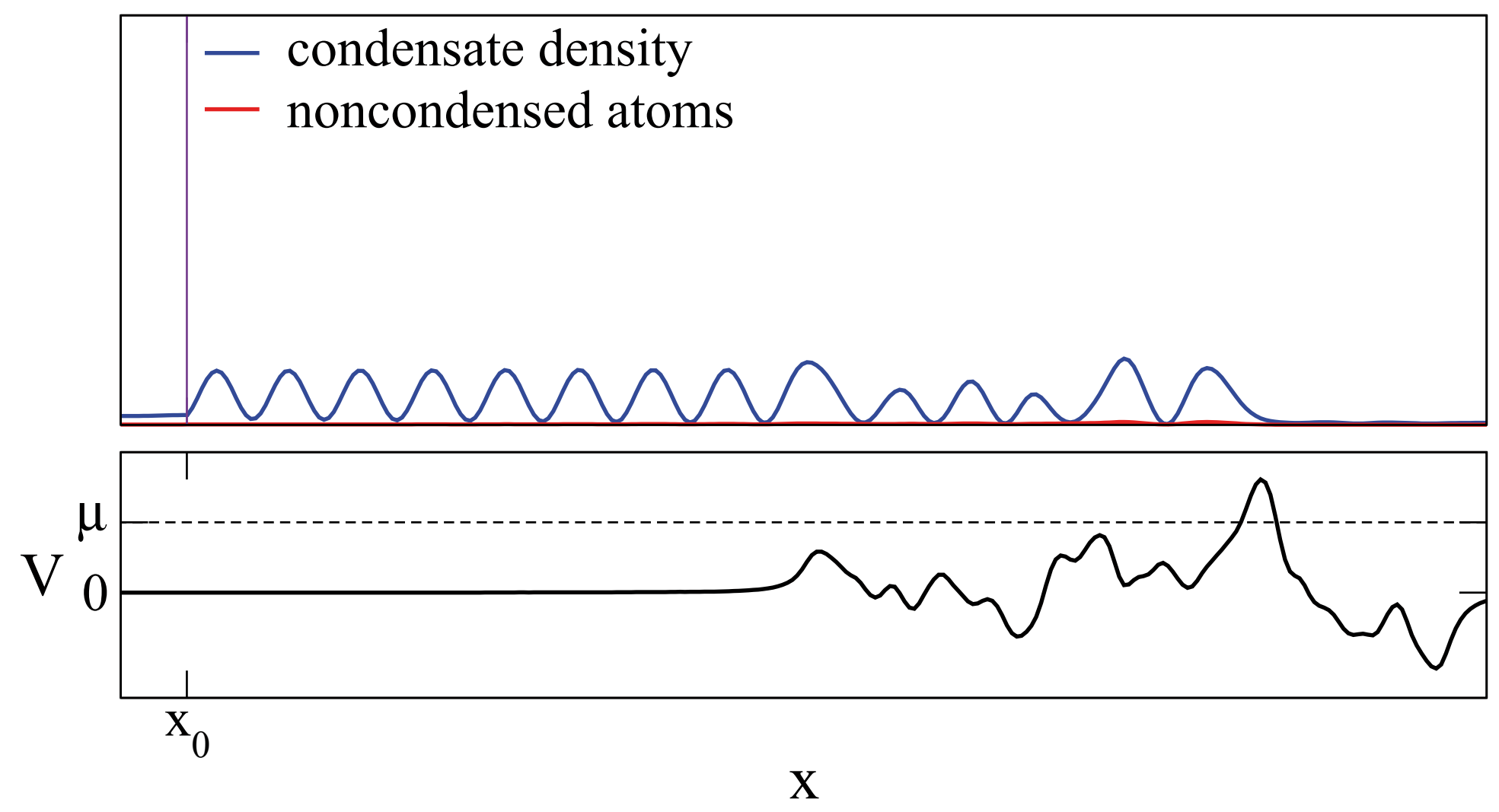




\section{Depletion in extended potentials}

Time-dependent scattering on the Gross-Pitaevskii level:

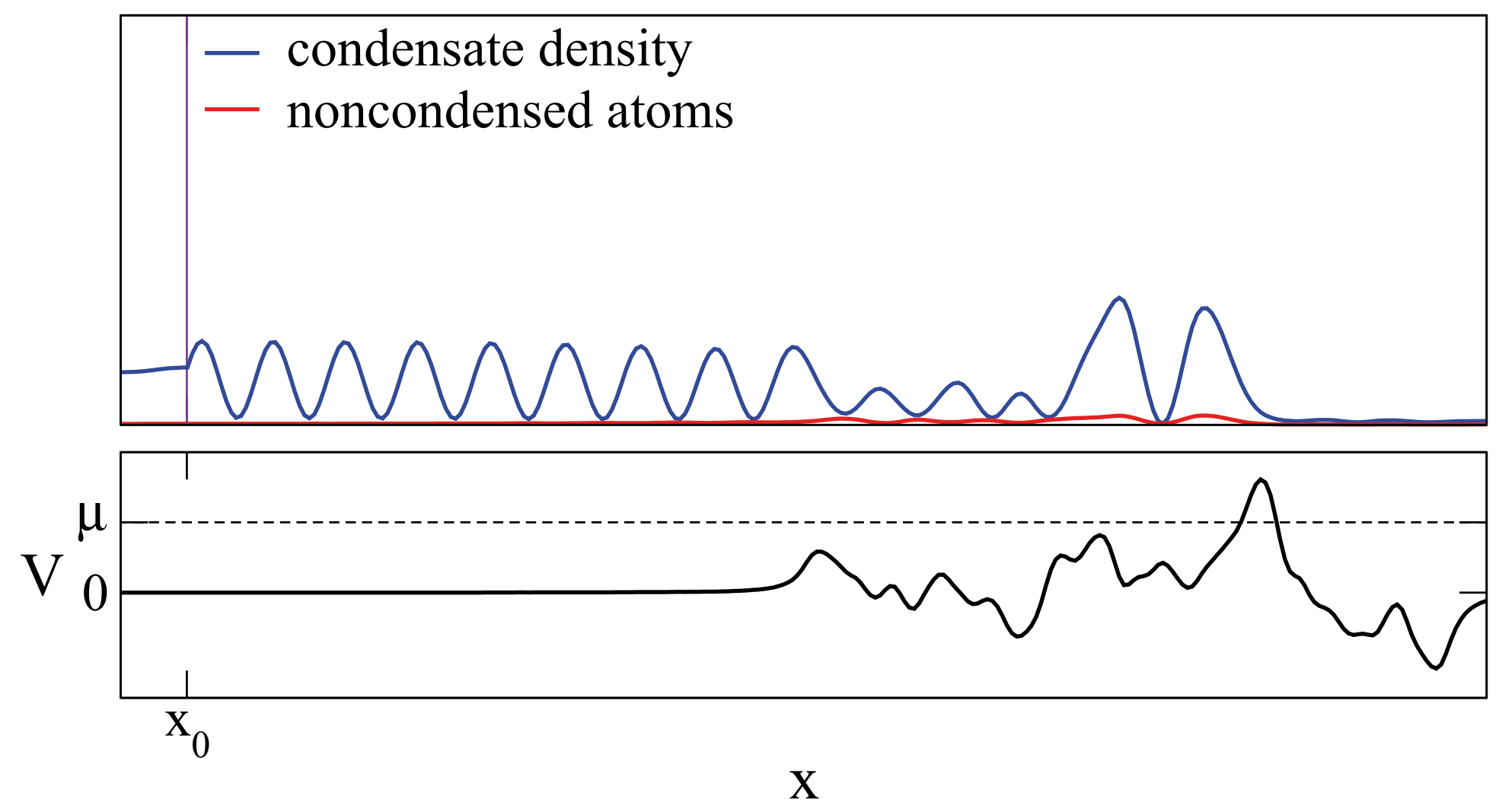




\section{Depletion in extended potentials}

Time-dependent scattering on the Gross-Pitaevskii level:

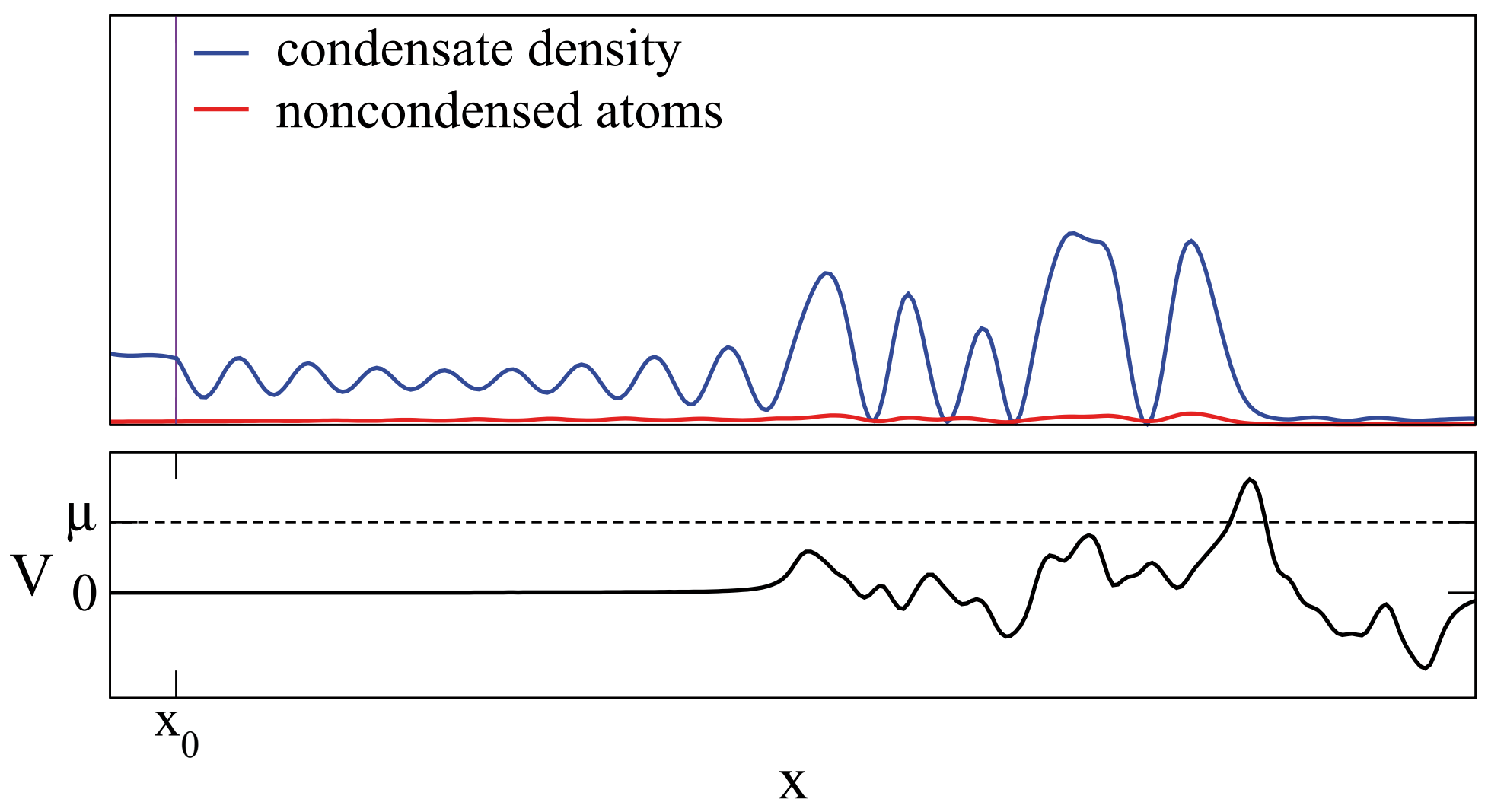




\section{Depletion in extended potentials}

Time-dependent scattering on the Gross-Pitaevskii level:
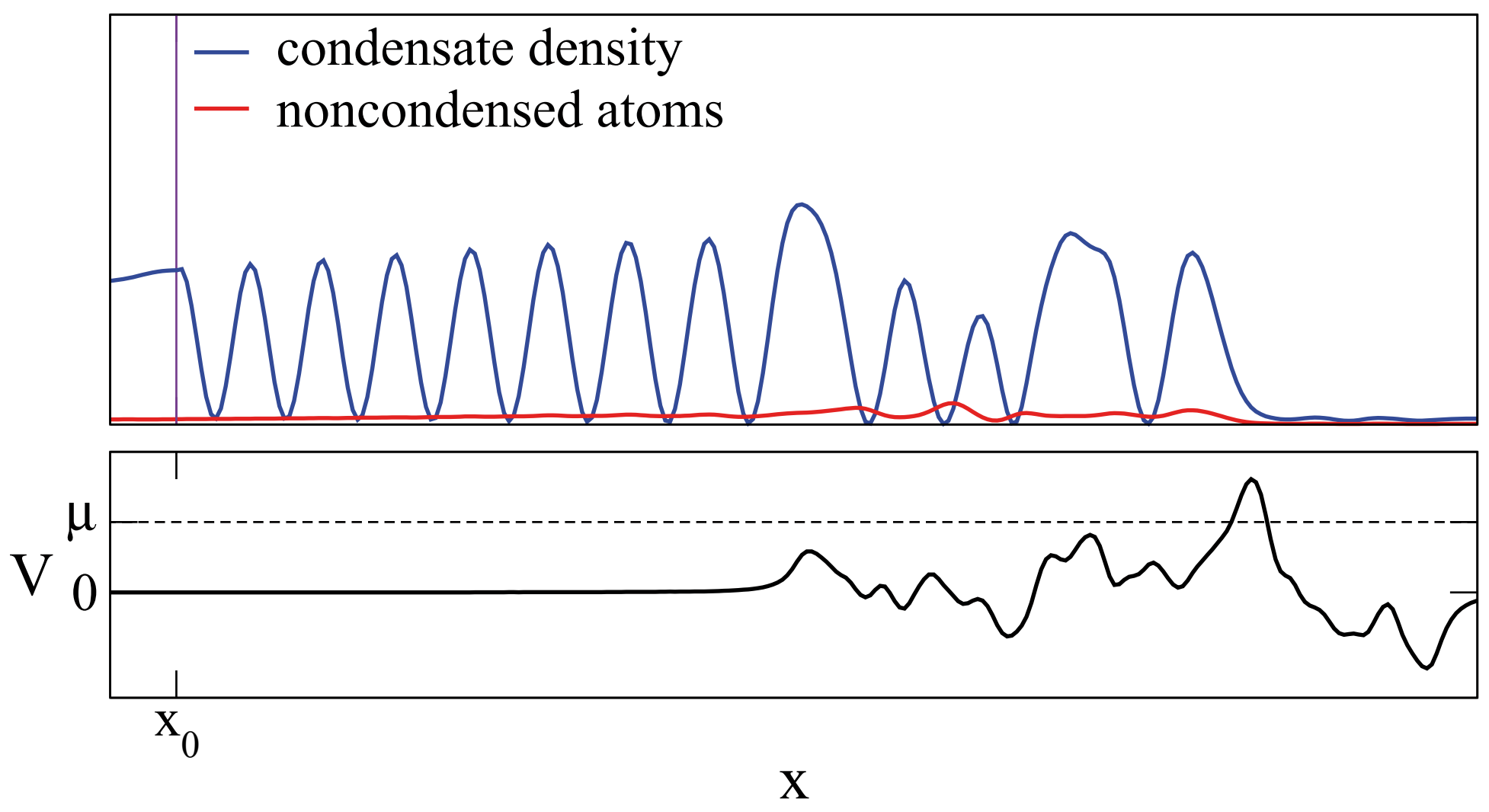


\section{Depletion in extended potentials}

Time-dependent scattering on the Gross-Pitaevskii level:

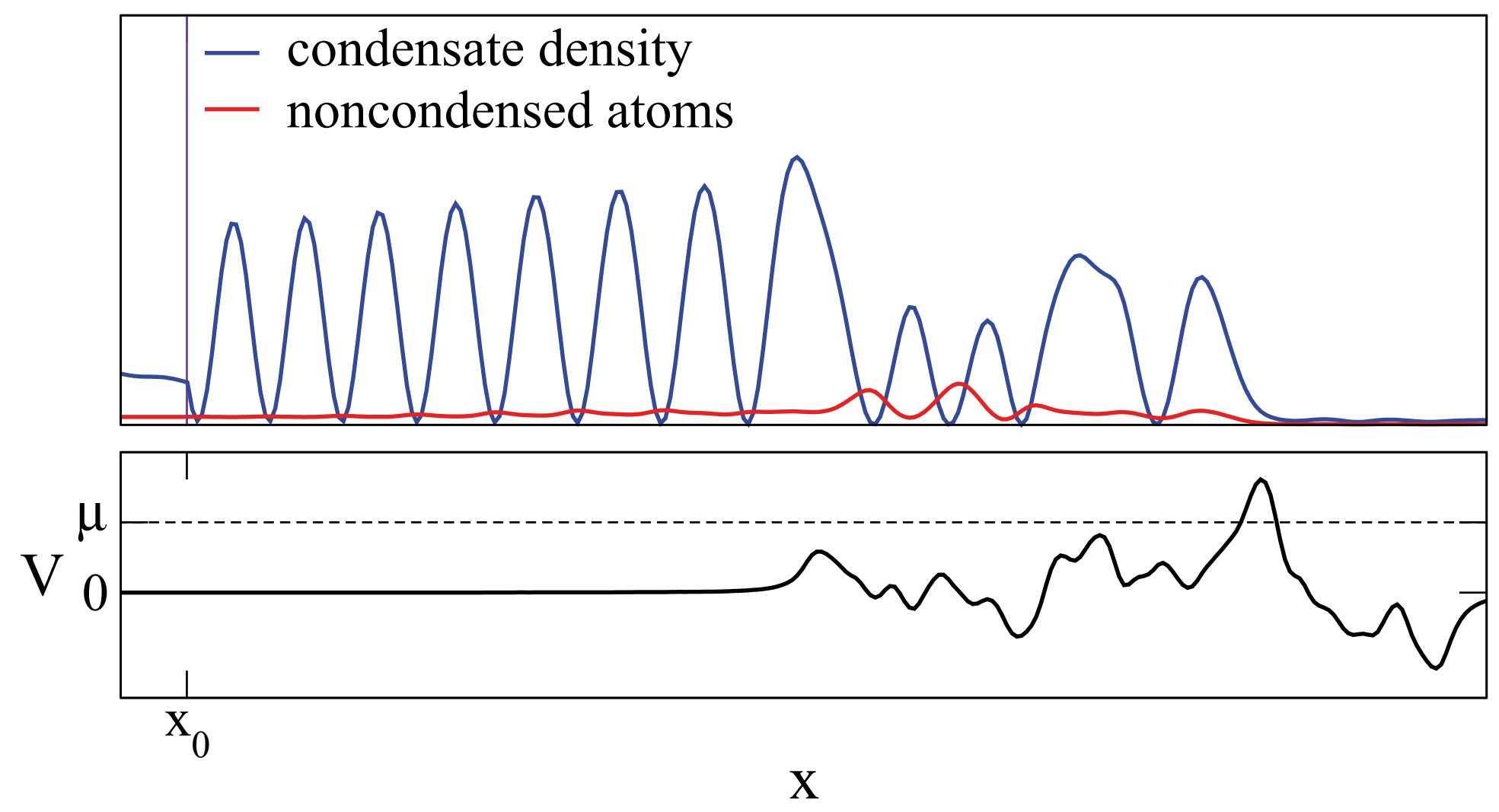




\section{Depletion in extended potentials}

Time-dependent scattering on the Gross-Pitaevskii level:

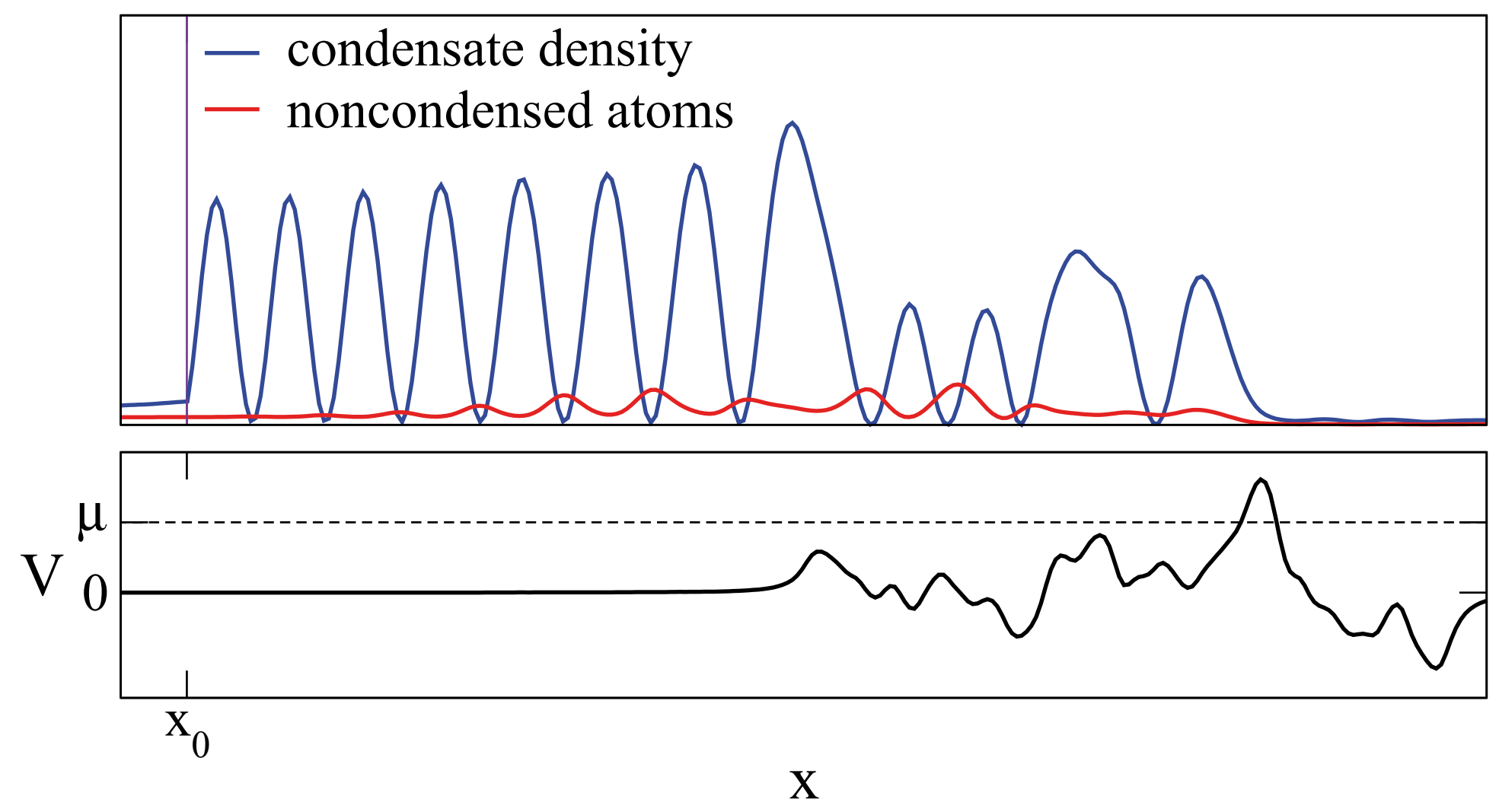




\section{Depletion in extended potentials}

Time-dependent scattering on the Gross-Pitaevskii level:

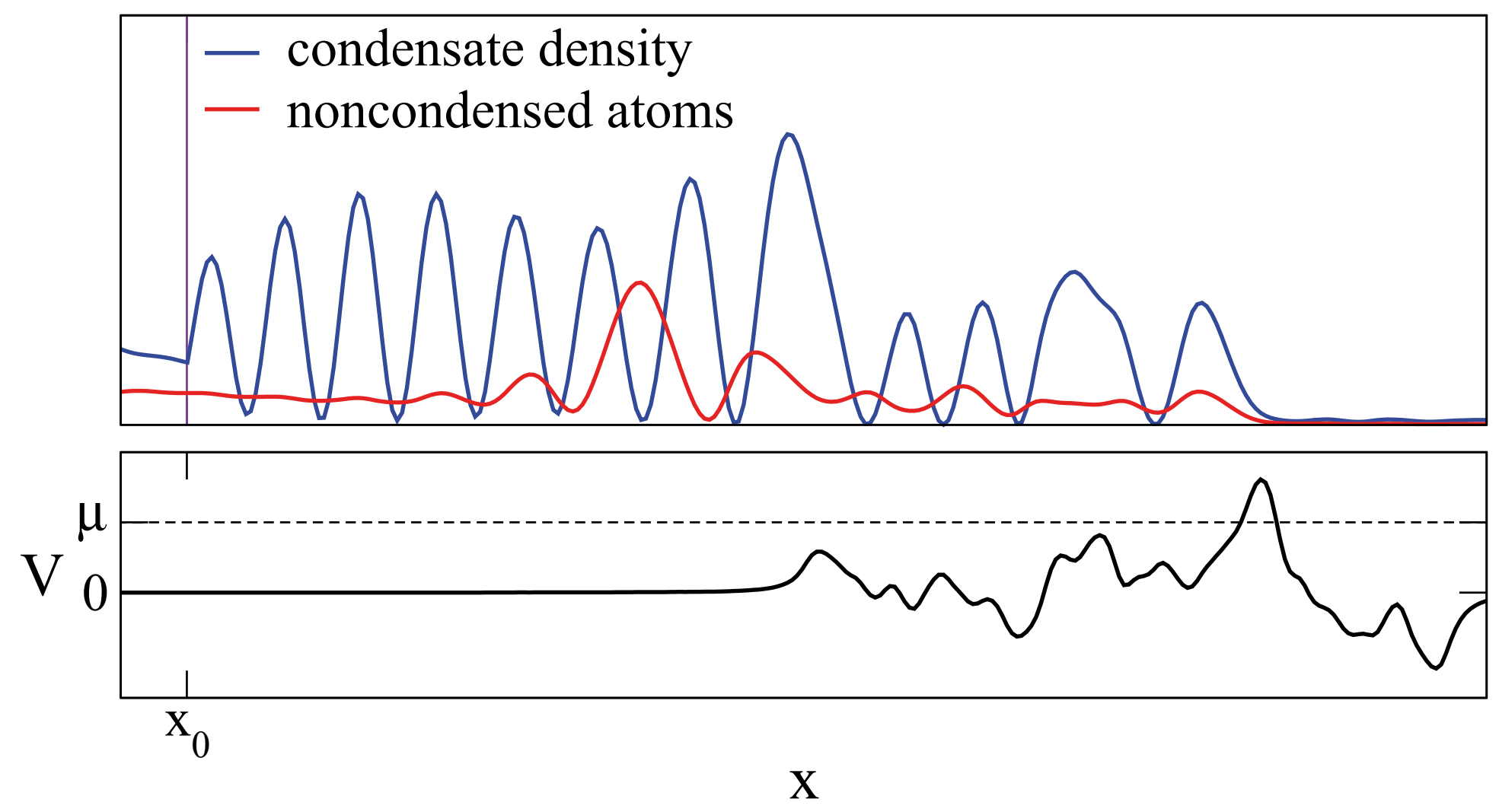




\section{Depletion in extended potentials}

Time-dependent scattering on the Gross-Pitaevskii level:
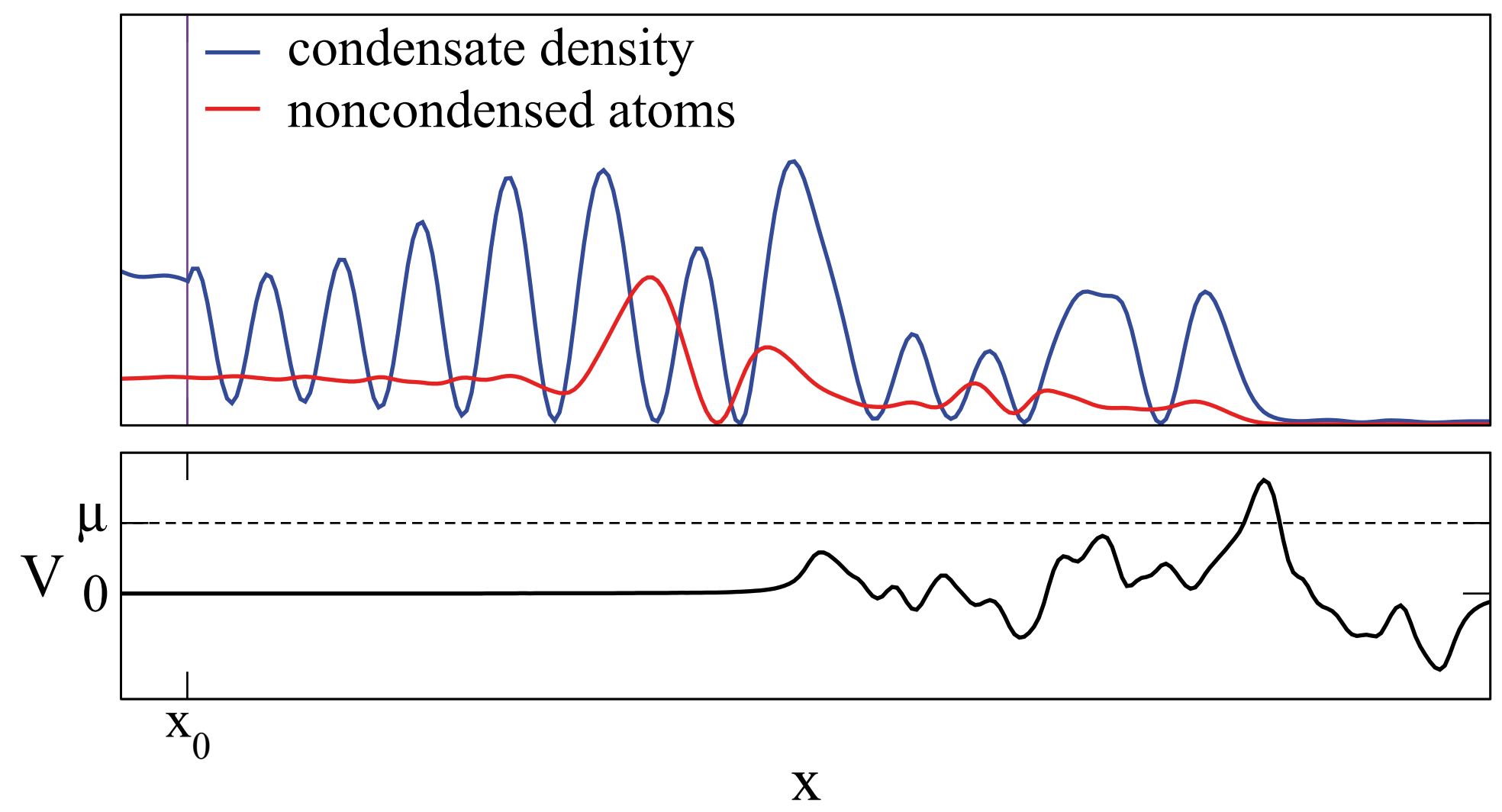


\section{Depletion in extended potentials}

Time-dependent scattering on the Gross-Pitaevskii level:
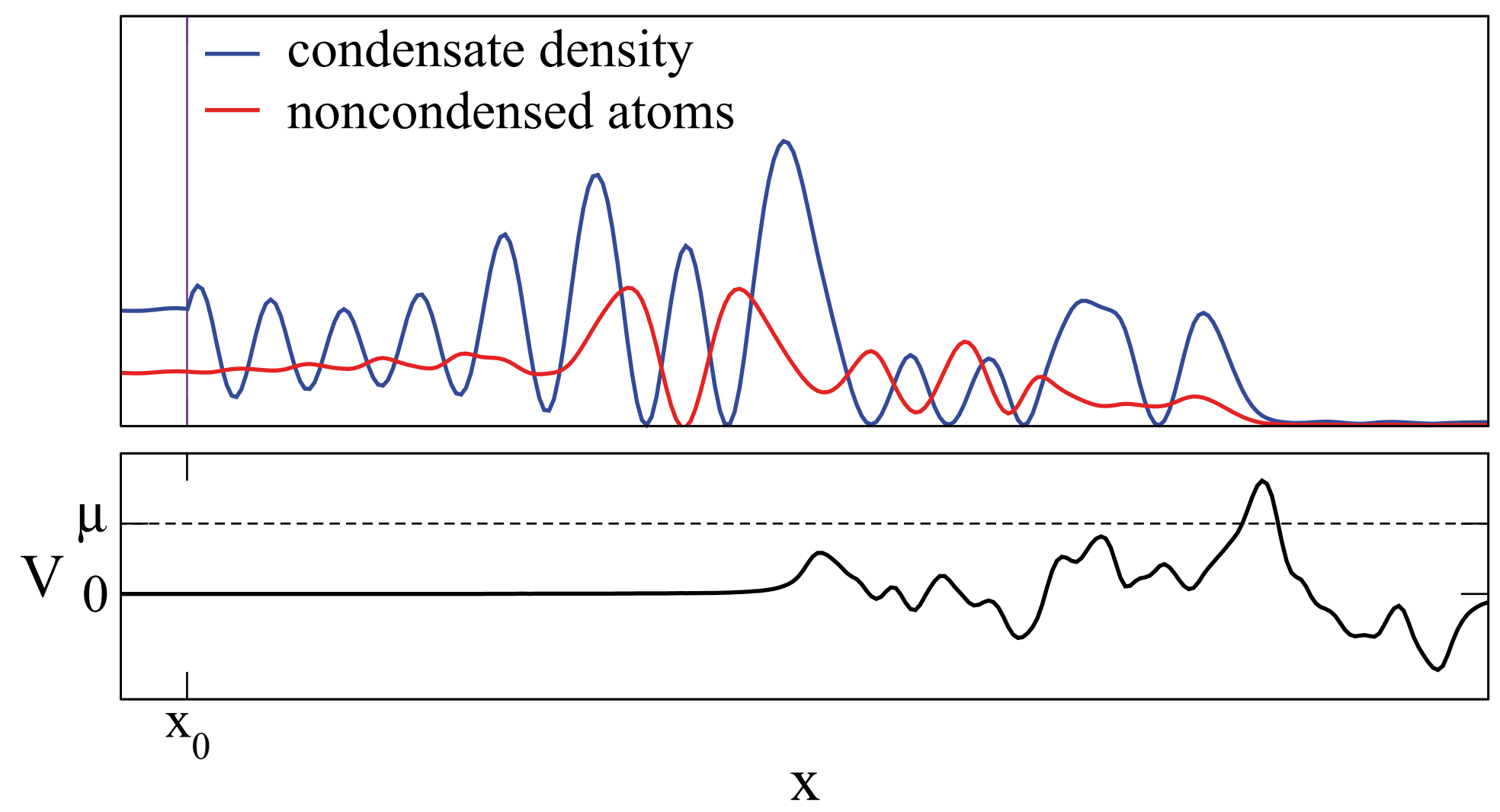


\section{Depletion in extended potentials}

Time-dependent scattering on the Gross-Pitaevskii level:

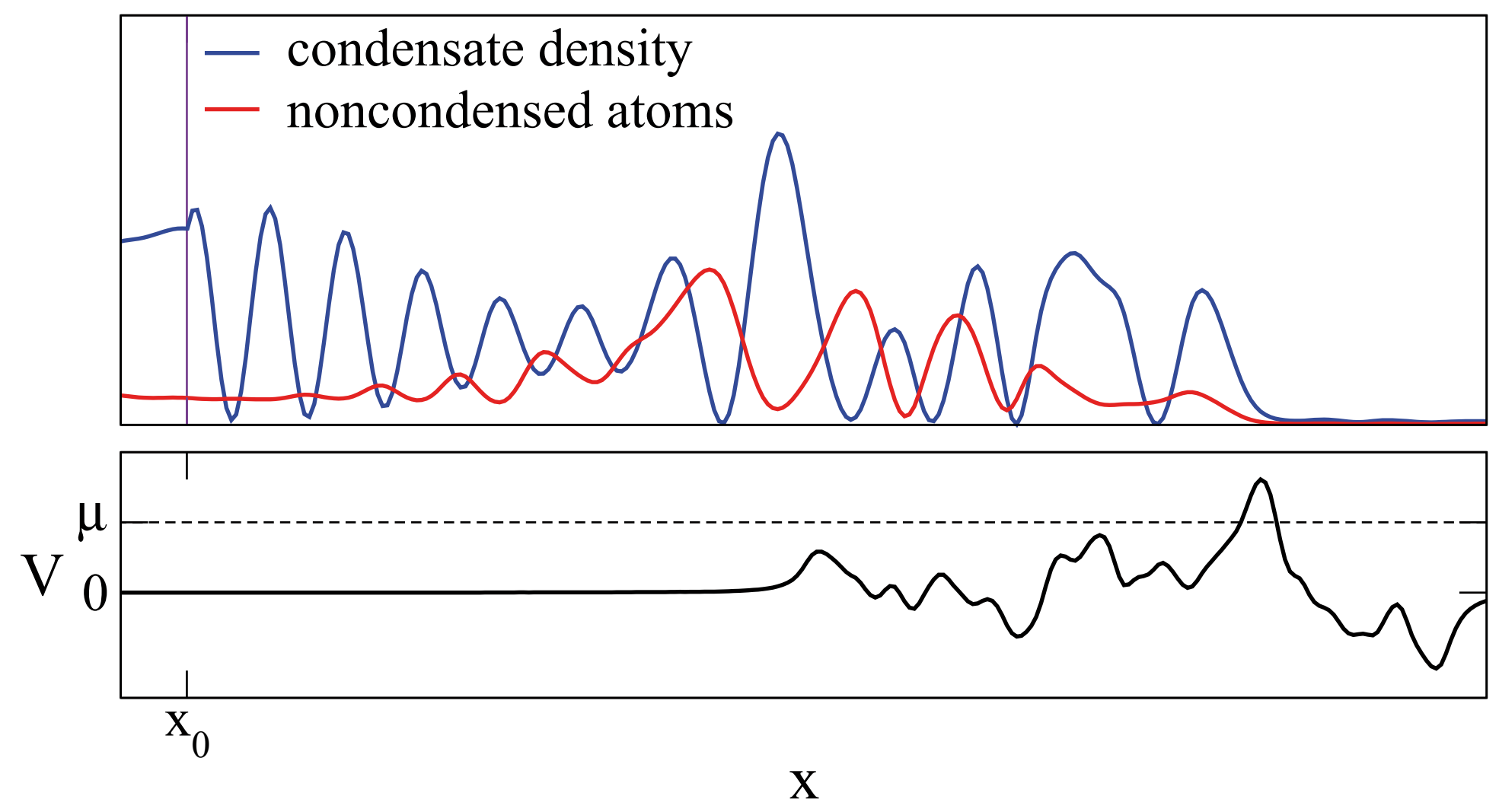




\section{Depletion in extended potentials}

Time-dependent scattering on the Gross-Pitaevskii level:

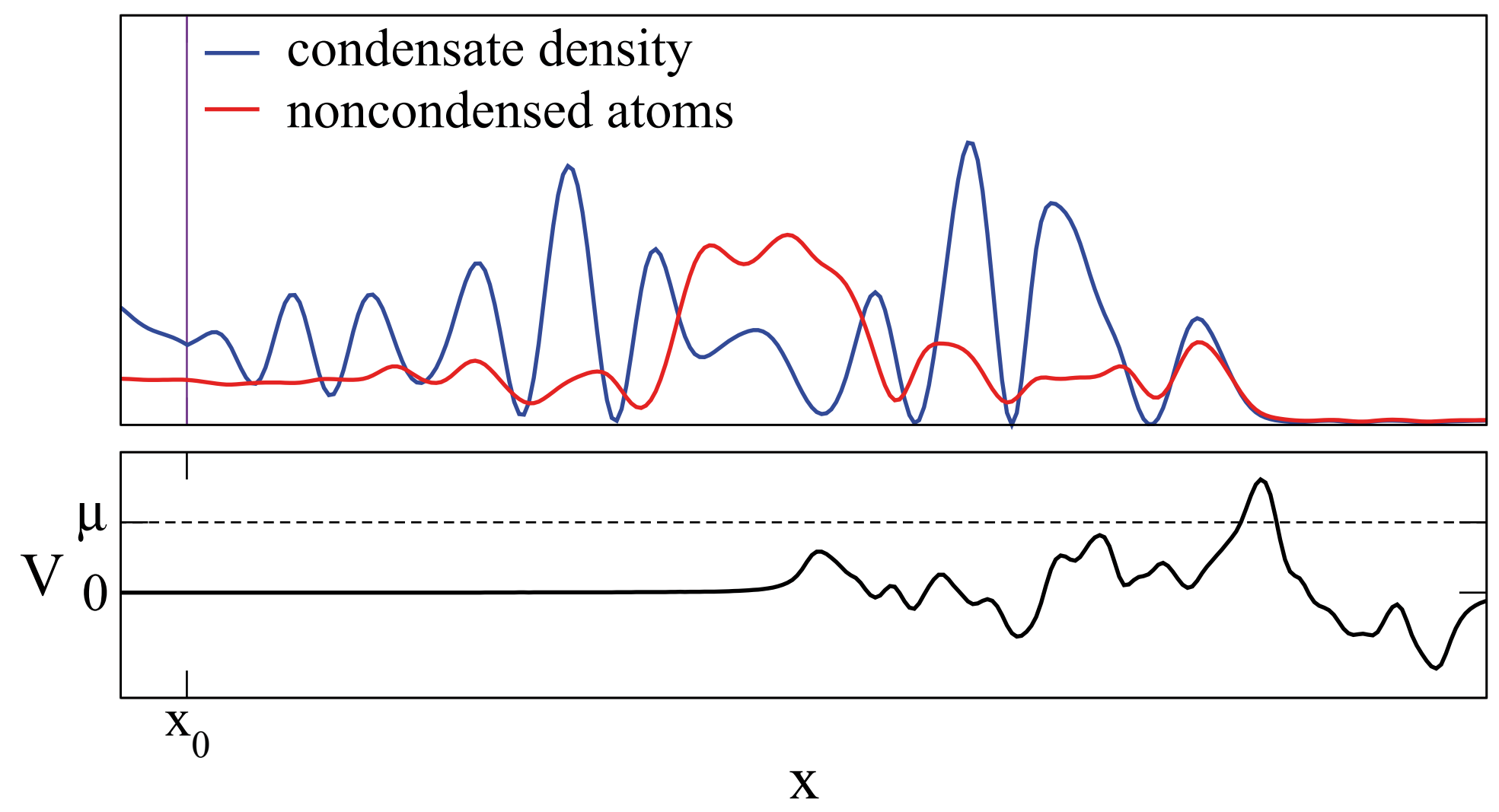




\section{Depletion in extended potentials}

Time-dependent scattering on the Gross-Pitaevskii level:

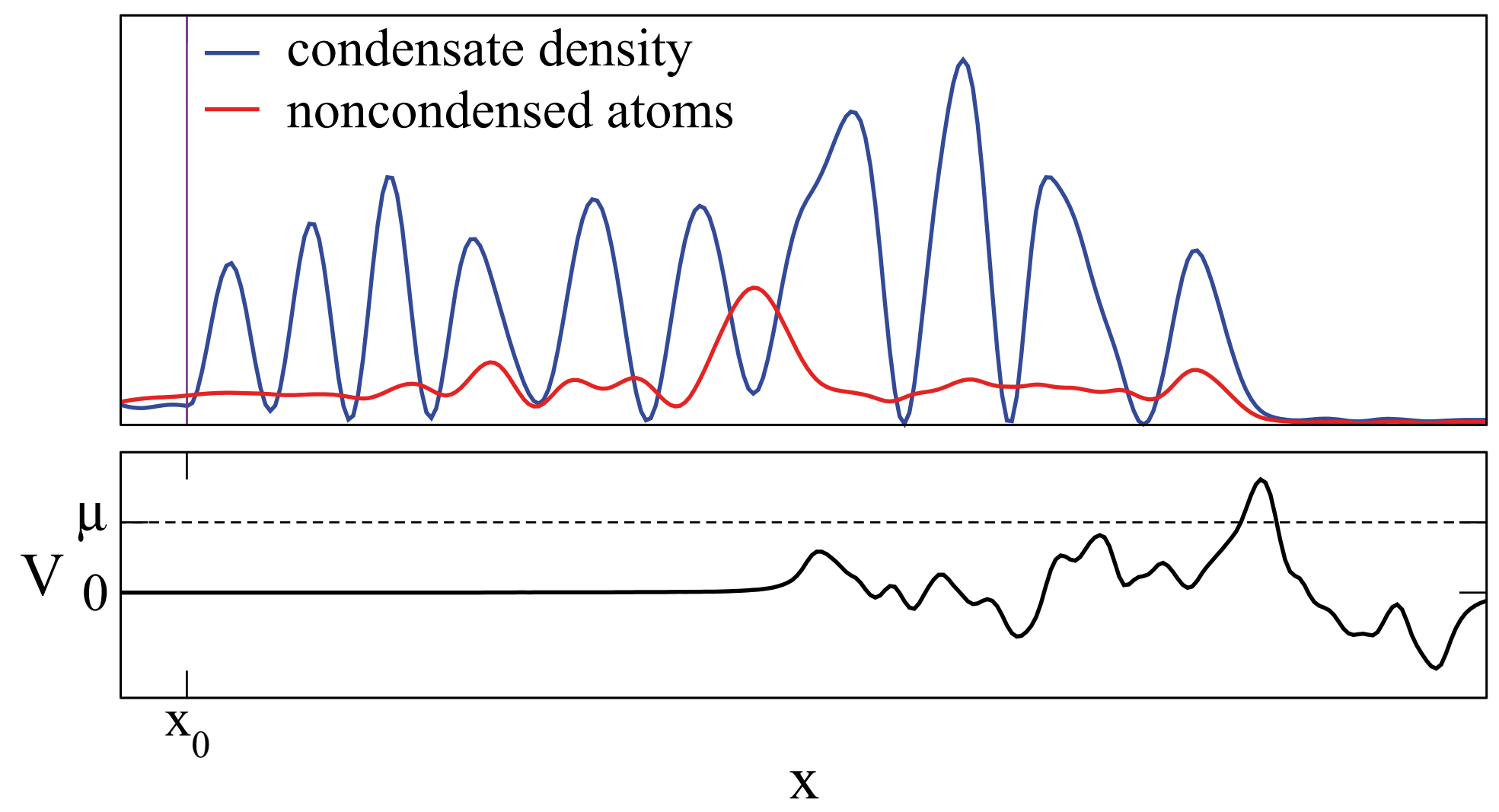




\section{Depletion in extended potentials}

Time-dependent scattering on the Gross-Pitaevskii level:

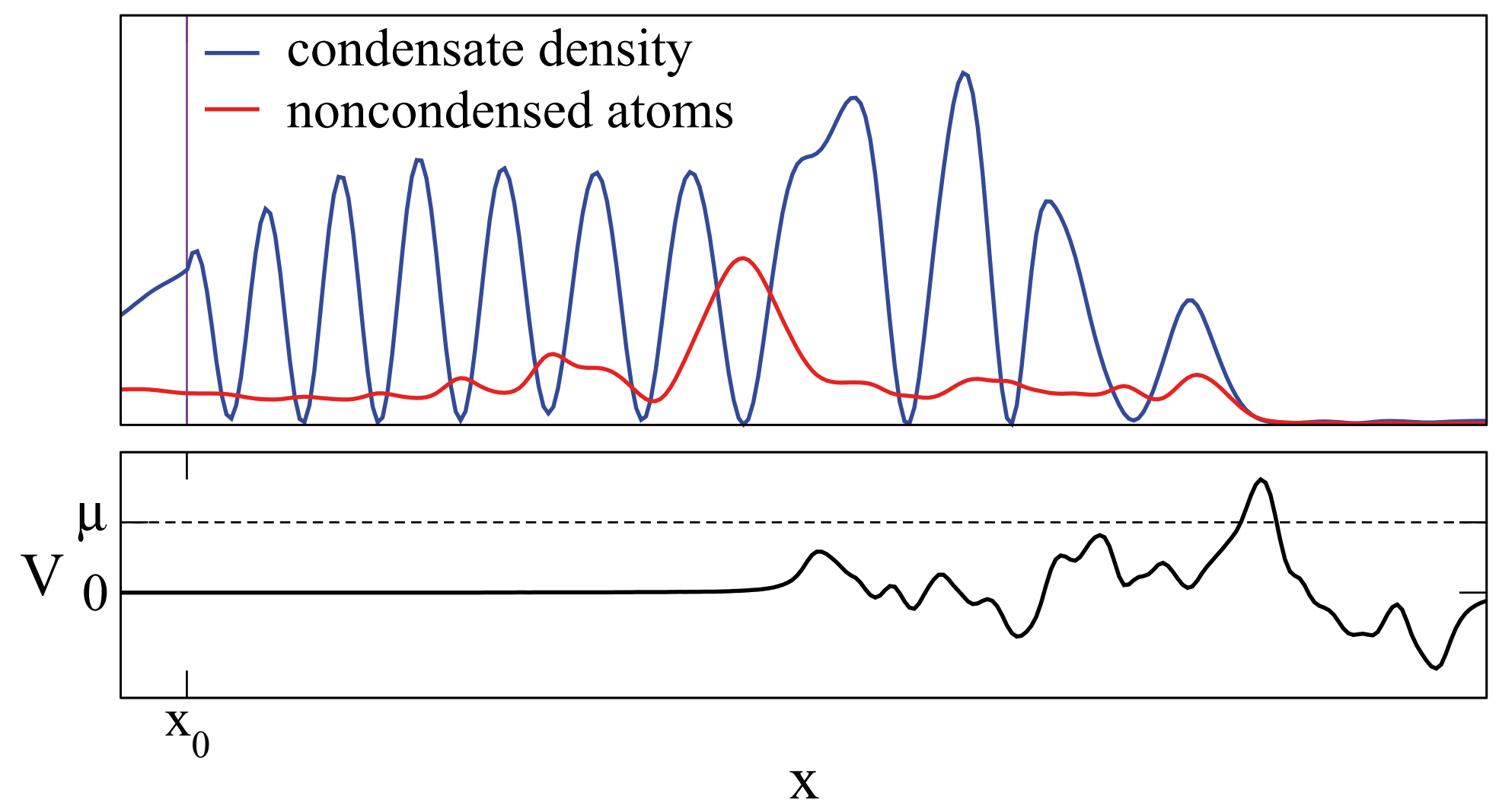




\section{Depletion in extended potentials}

Time-dependent scattering on the Gross-Pitaevskii level:
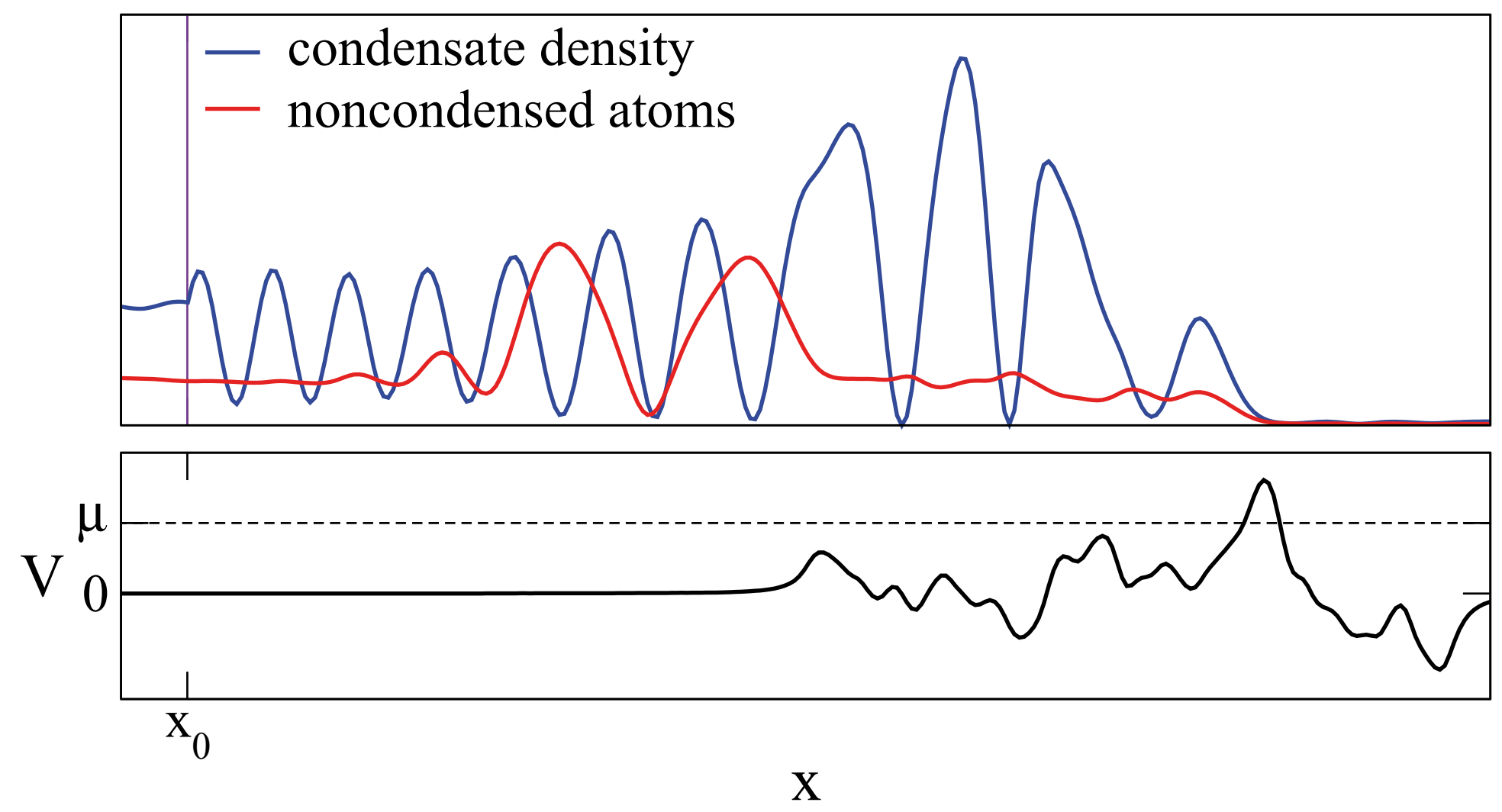


\section{Depletion in extended potentials}

Time-dependent scattering on the Gross-Pitaevskii level:
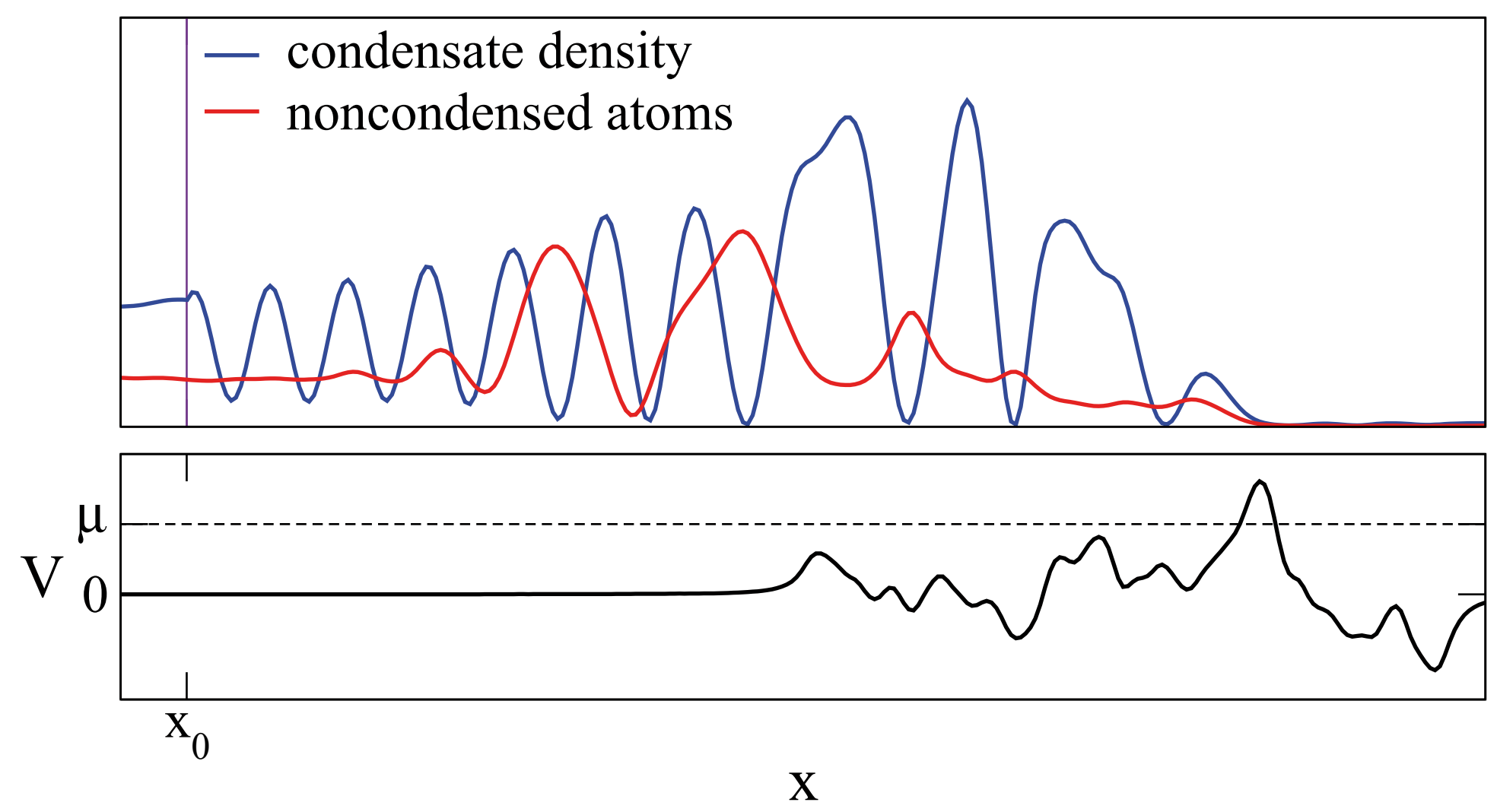


\section{Depletion in extended potentials}

Time-dependent scattering on the Gross-Pitaevskii level:

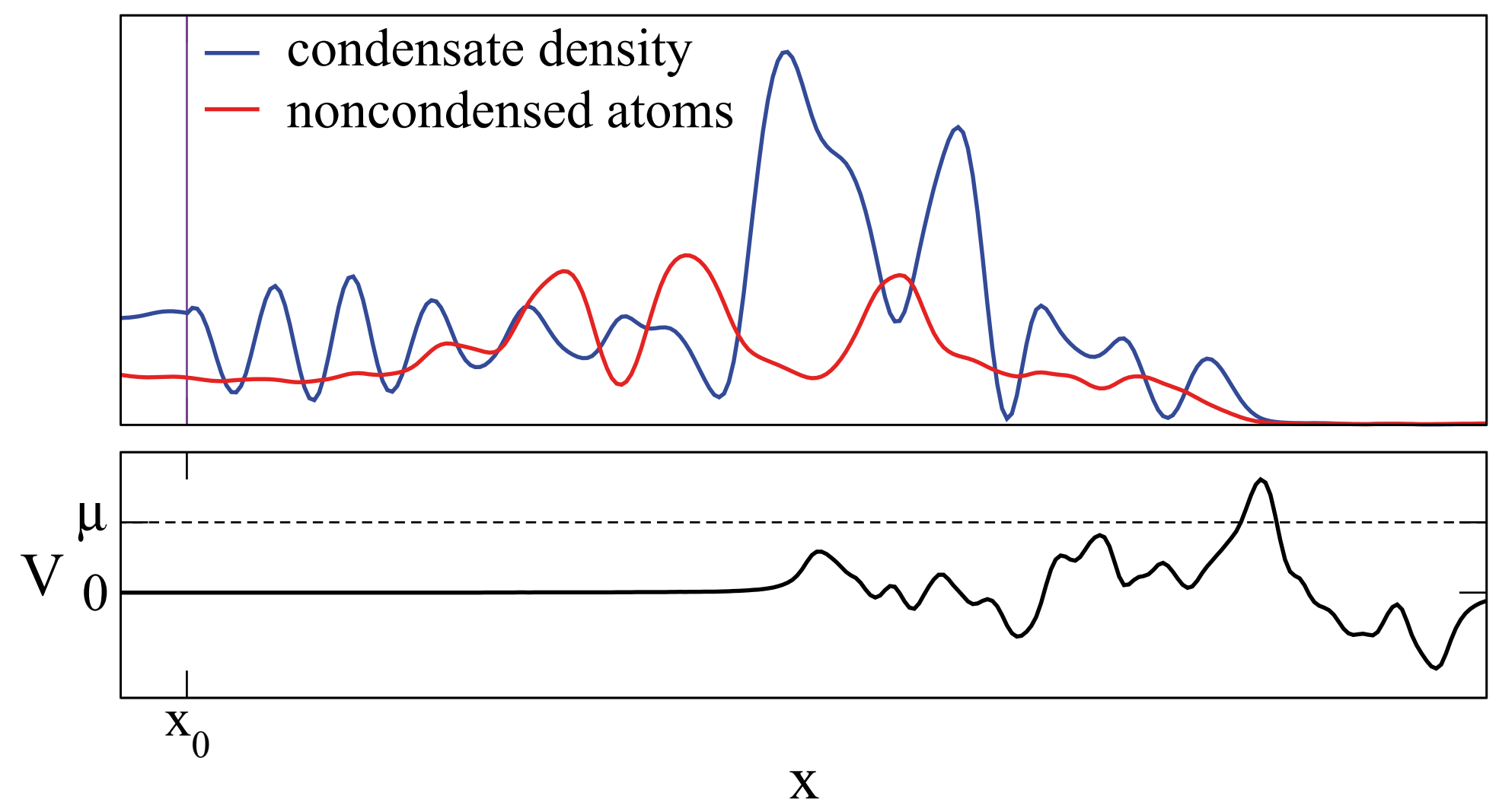




\section{Depletion in extended potentials}

Time-dependent scattering on the Gross-Pitaevskii level:
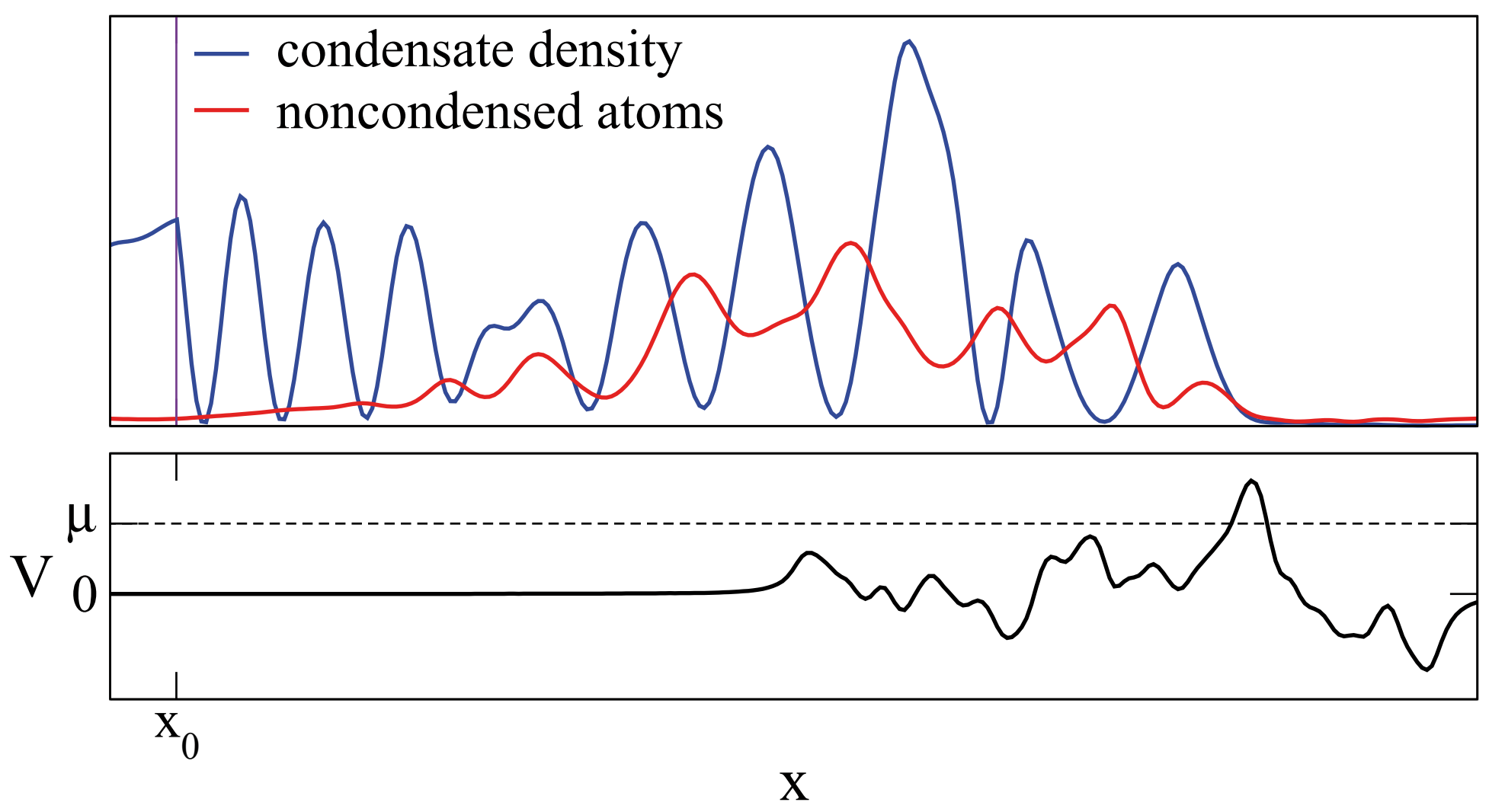


\section{Depletion in extended potentials}

Time-dependent scattering on the Gross-Pitaevskii level:
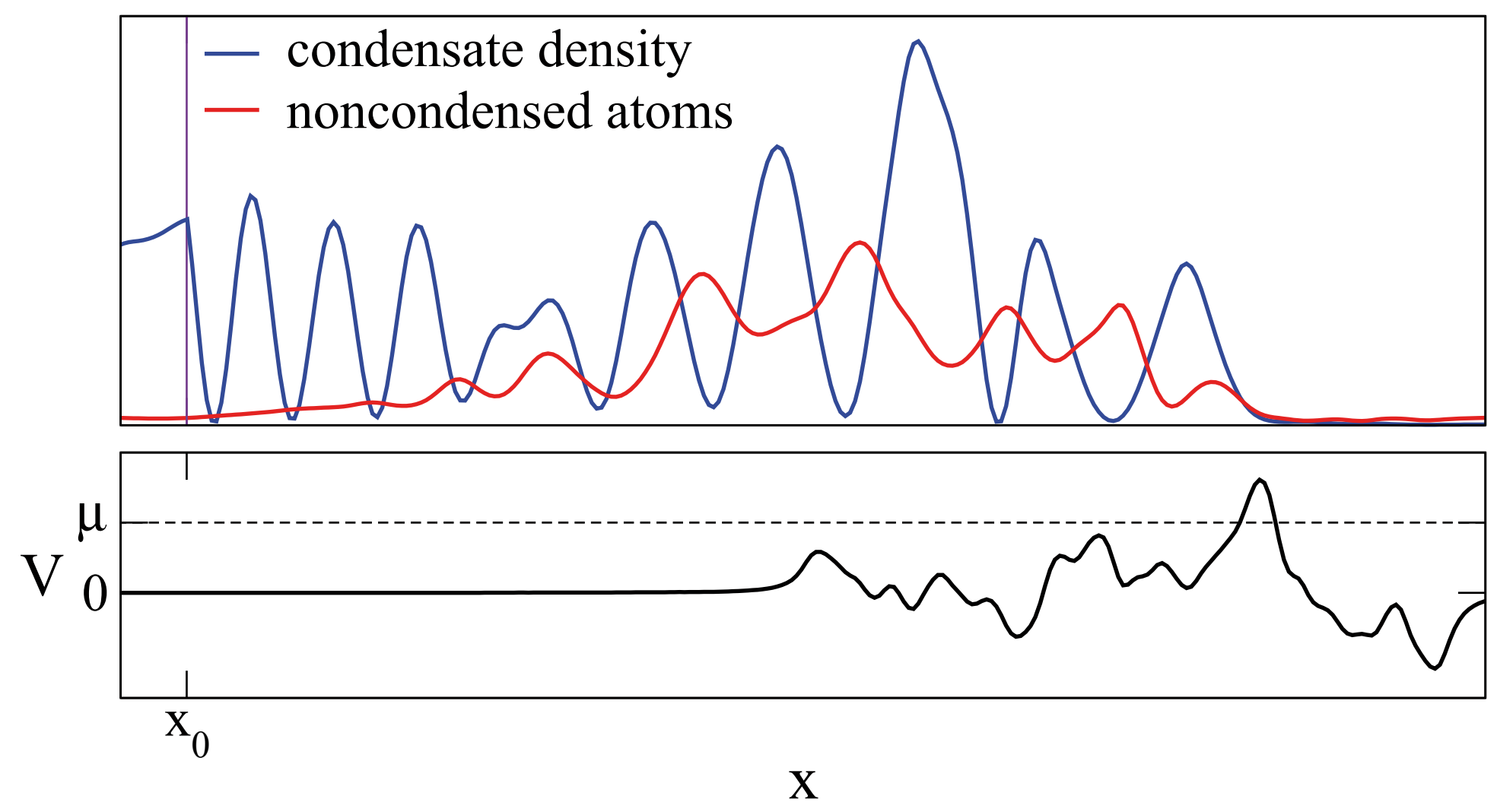

$\longrightarrow$ strong depletion, breakdown of Gross-Pitaevskii description 


\section{Depletion in extended potentials}

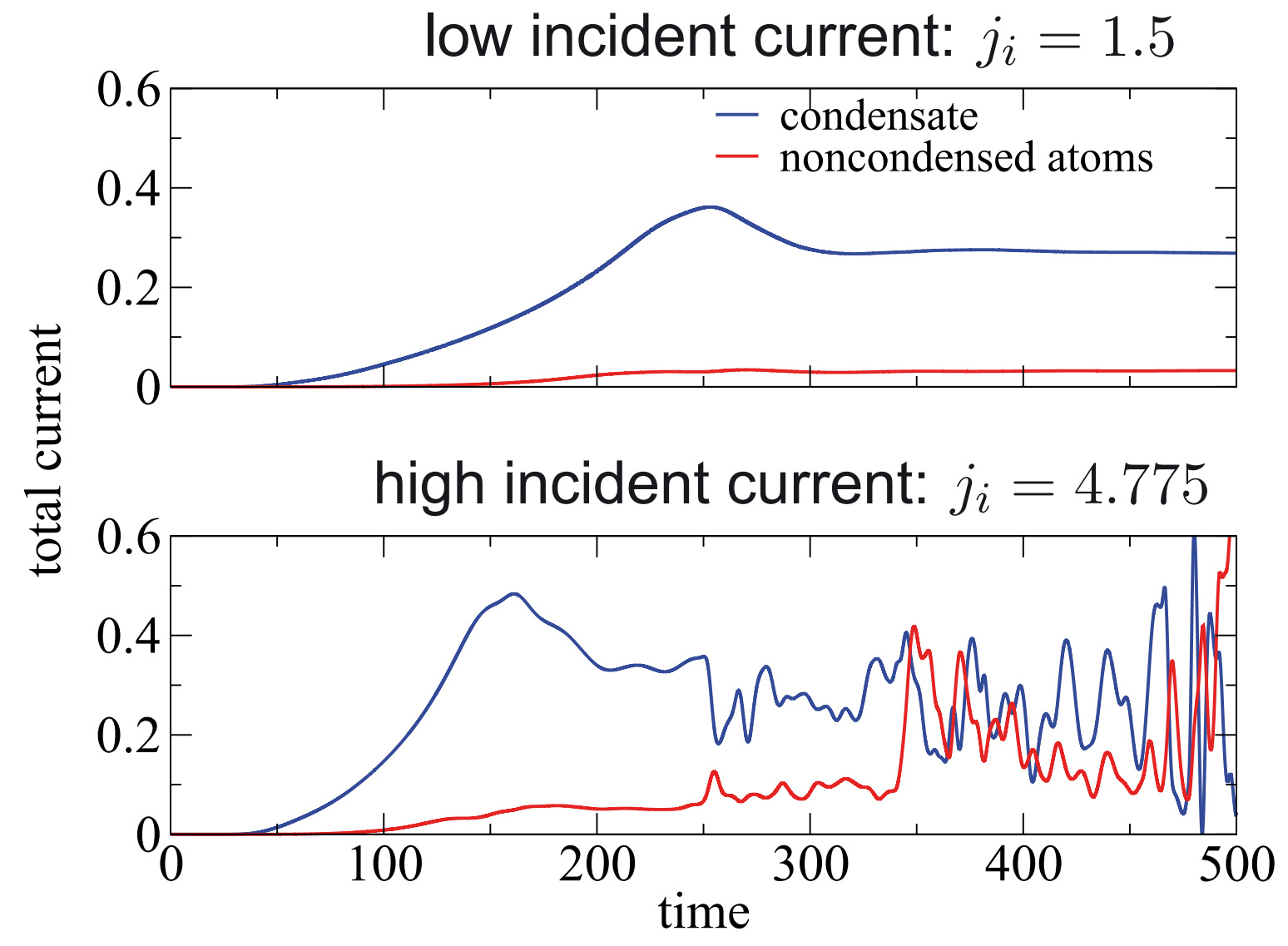

T. Ernst, T. Paul, and P.S., PRA 81, 0136431 (2010) 


\section{Depletion in extended potentials}

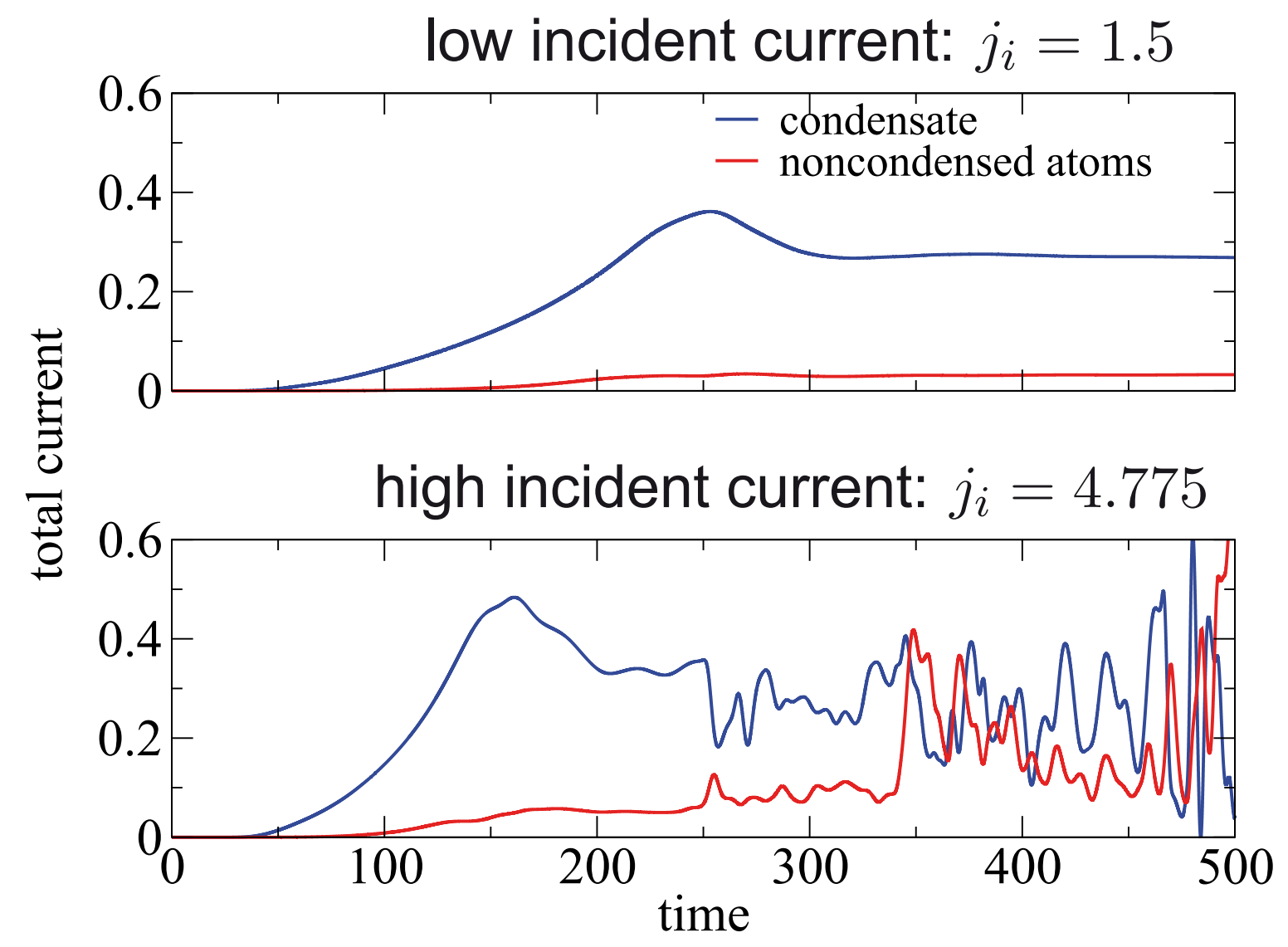

T. Ernst, T. Paul, and P.S., PRA 81, 0136431 (2010)

Alternative: truncated Wigner approach

R. G. Scott and D. A. W. Hutchinson, PRA 78, 063614 (2008) 


\section{Transport through 1D disorder potentials}

Consider spatially correlated disorder potentials with

- Gaussian correlation

- Lorentzian correlation

$\rightarrow$ disorder on atom chips

- Bessel-type correlation

$\rightarrow$ optical speckle fields
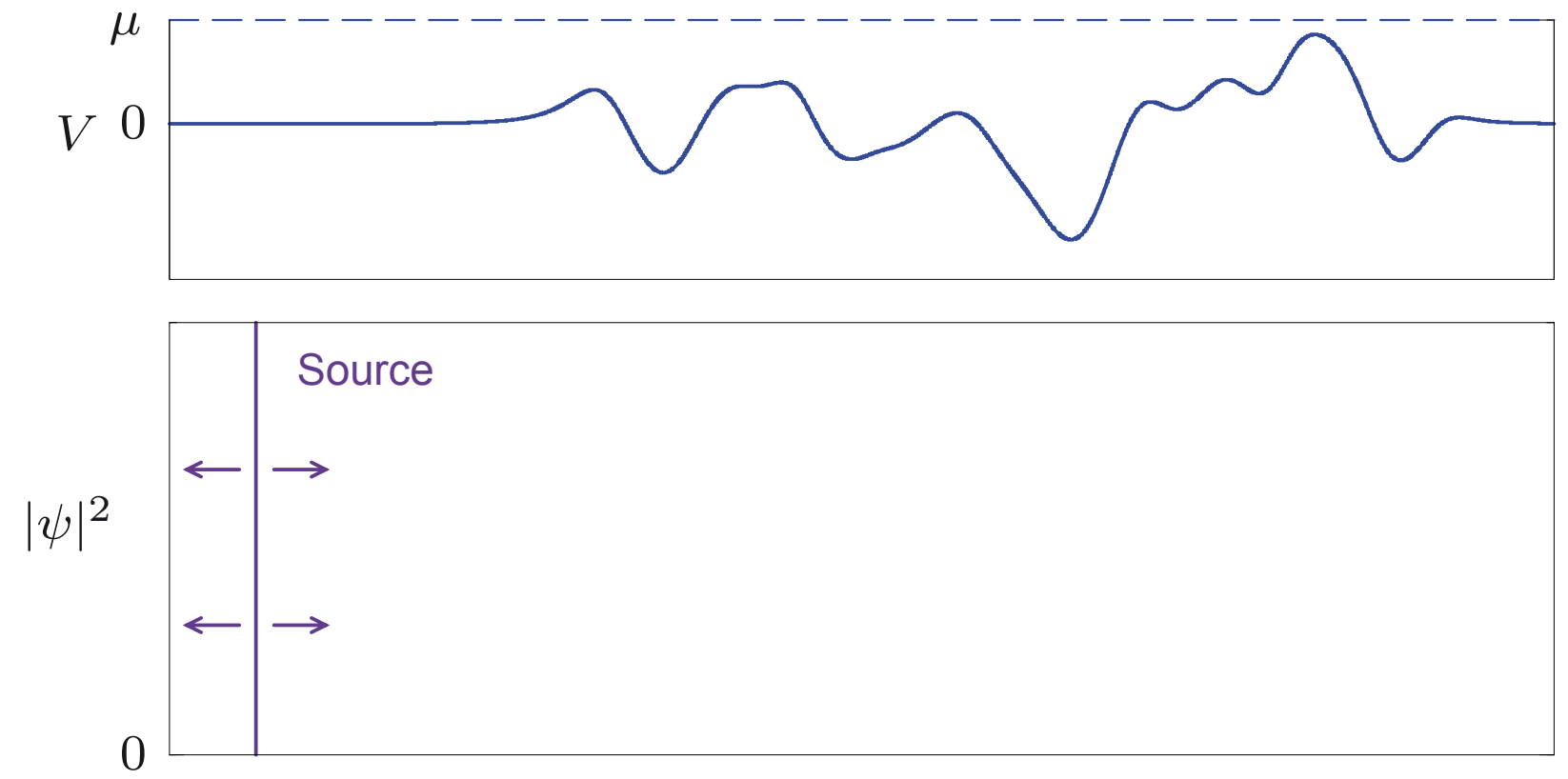


\section{Transport through 1D disorder potentials}

Negligible interaction between the atoms: Anderson localization

$\rightarrow$ exponential decrease of the average transmission with the length $L$ of the disorder region

$\rightarrow$ lognormal-type probability distribution for the transmission $T$ at fixed length $L$ :

$$
P(\ln T)=\sqrt{\frac{L_{\mathrm{loc}}}{4 \pi L}} \exp \left[-\frac{L_{\mathrm{loc}}}{4 L}\left(\frac{L}{L_{\mathrm{loc}}}+\ln T\right)^{2}\right]
$$

Dorokhov (1982); Mello, Pereyra, Kumar (1988); Pichard (1991) 


\section{Transport through 1D disorder potentials}

Negligible interaction between the atoms: Anderson localization

$\rightarrow$ exponential decrease of the average transmission with the length $L$ of the disorder region

$\rightarrow$ lognormal-type probability distribution for the transmission $T$ at fixed length $L$ :

$$
P(\ln T)=\sqrt{\frac{L_{\mathrm{loc}}}{4 \pi L}} \exp \left[-\frac{L_{\mathrm{loc}}}{4 L}\left(\frac{L}{L_{\mathrm{loc}}}+\ln T\right)^{2}\right]
$$

$\longrightarrow$ same expression $L_{\mathrm{loc}} \equiv L_{\mathrm{loc}}(\kappa)$ as for noninteracting atoms, with a "renormalized" wavenumber $\kappa=\sqrt{k^{2}-m g|\psi|^{2} / \hbar^{2}}$

T. Paul et al., PRL 98, 210602 (2007); PRA 80, 033615 (2009) 


\section{Lognormal distribution for speckle disorder}

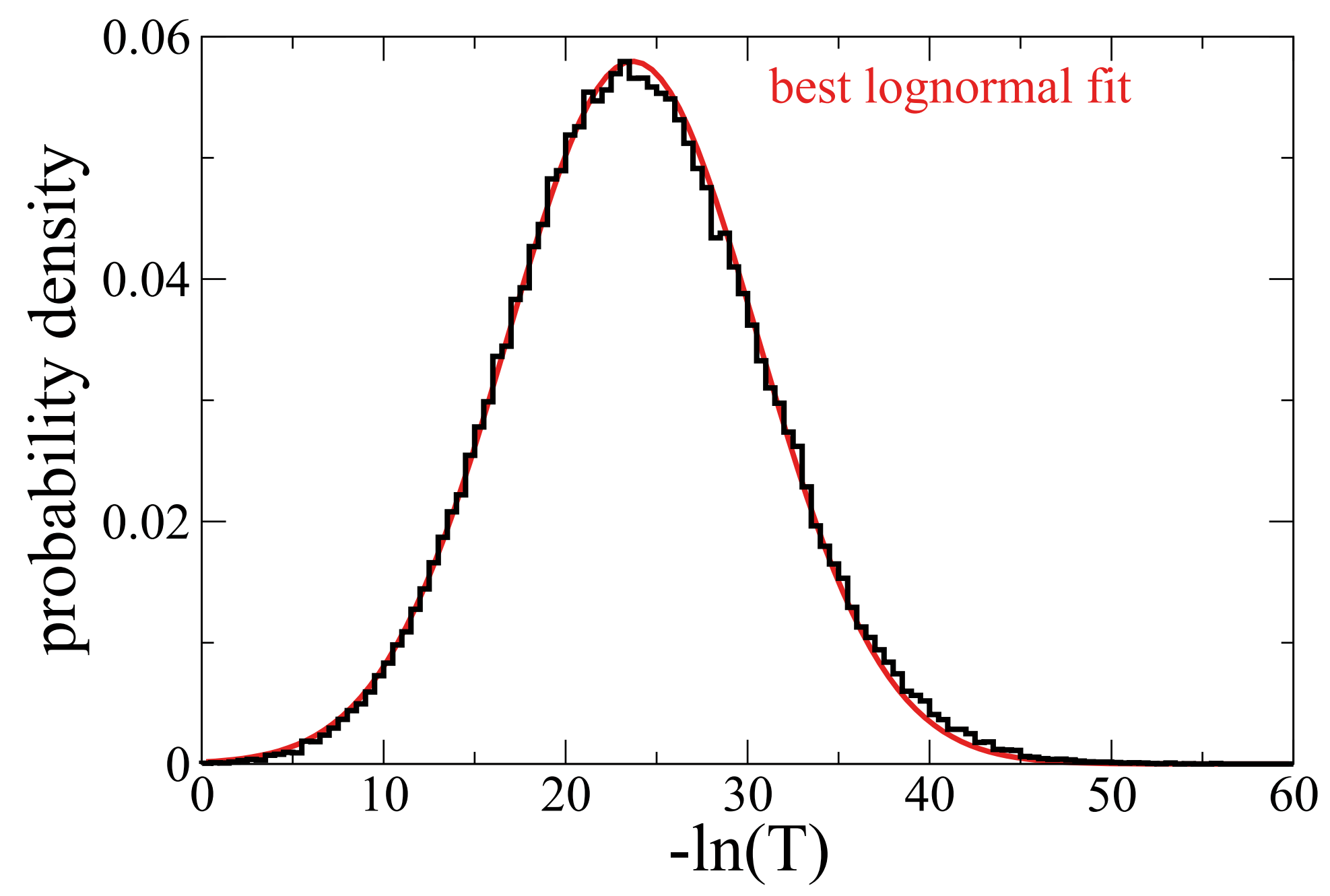




\section{Lognormal distribution for speckle disorder}

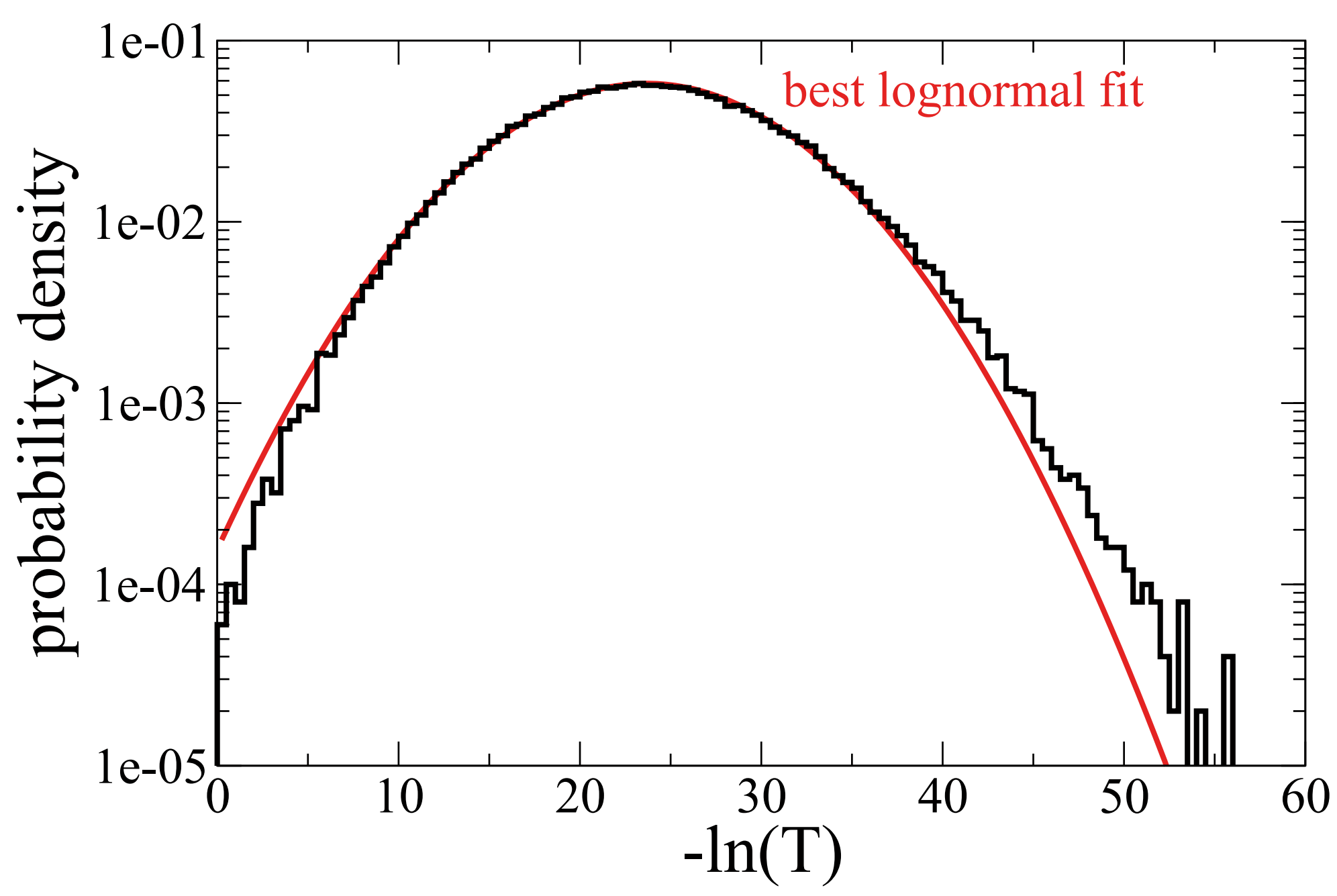




\section{Transport through 1D disorder potentials}

Finite interaction between the atoms:

$\rightarrow$ permanently time-dependent scattering, except for very short disorder samples

$\rightarrow$ compute time-averaged transmission $\bar{T}=\frac{1}{\Delta t} \int_{t_{0}}^{t_{0}+\Delta t} T(t) d t$

$\rightarrow$ algebraic (Ohm-like) decrease of the average transmission:

$$
\bar{T} \simeq \frac{1}{1+L / L_{0}}
$$

$\longrightarrow$ signature for incoherent transport

T. Paul, P. Leboeuf, N. Pavloff, K. Richter, and P.S., PRA 72, 063621 (2005) 


\section{Two-dimensional disorder}

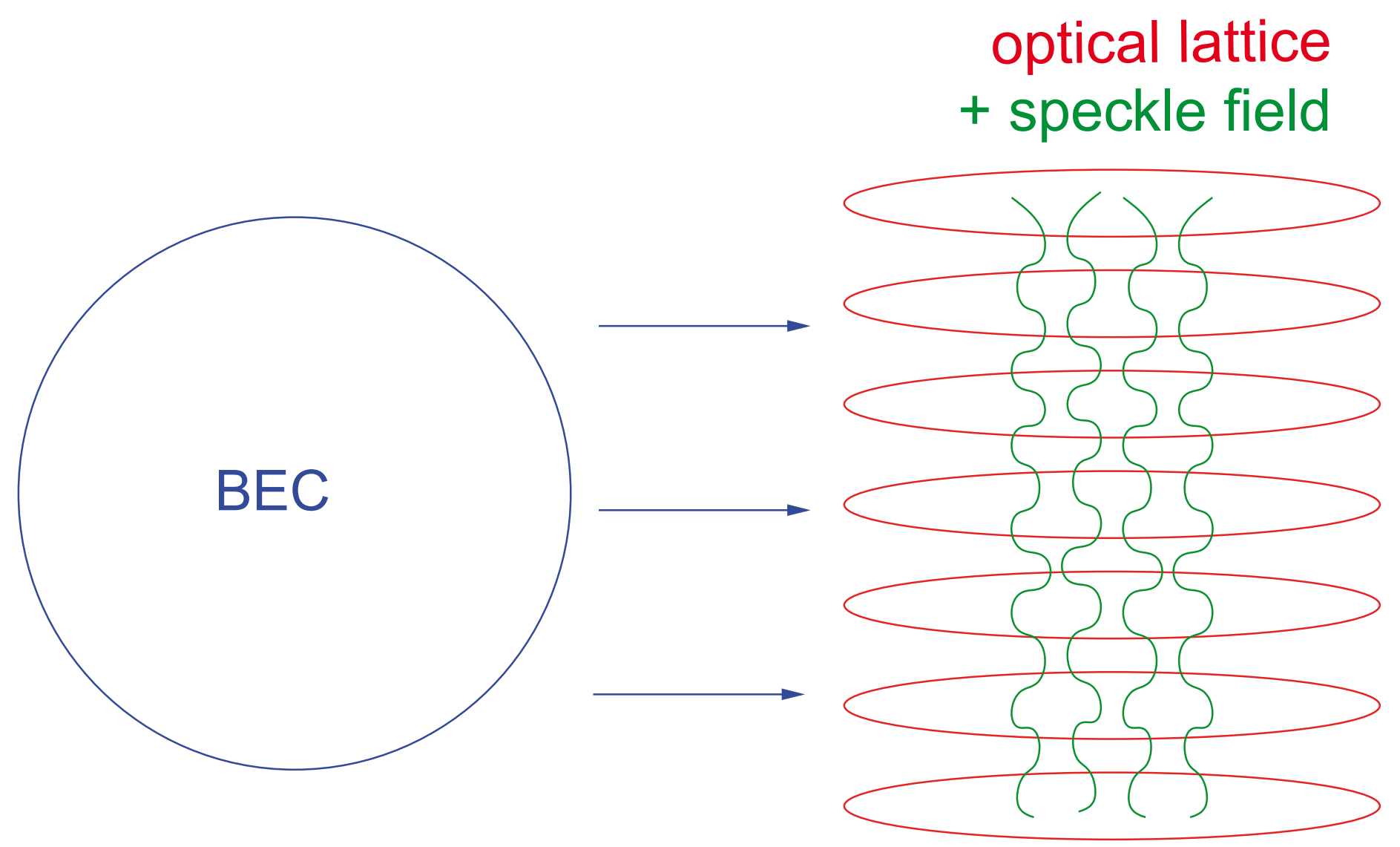

$\longrightarrow$ measure angle-resolved flux of backscattered atoms 


\section{Weak localization in two-dimensional disorder}

$\rightarrow$ Constructive interference between reflected paths and their time-reversed counterparts
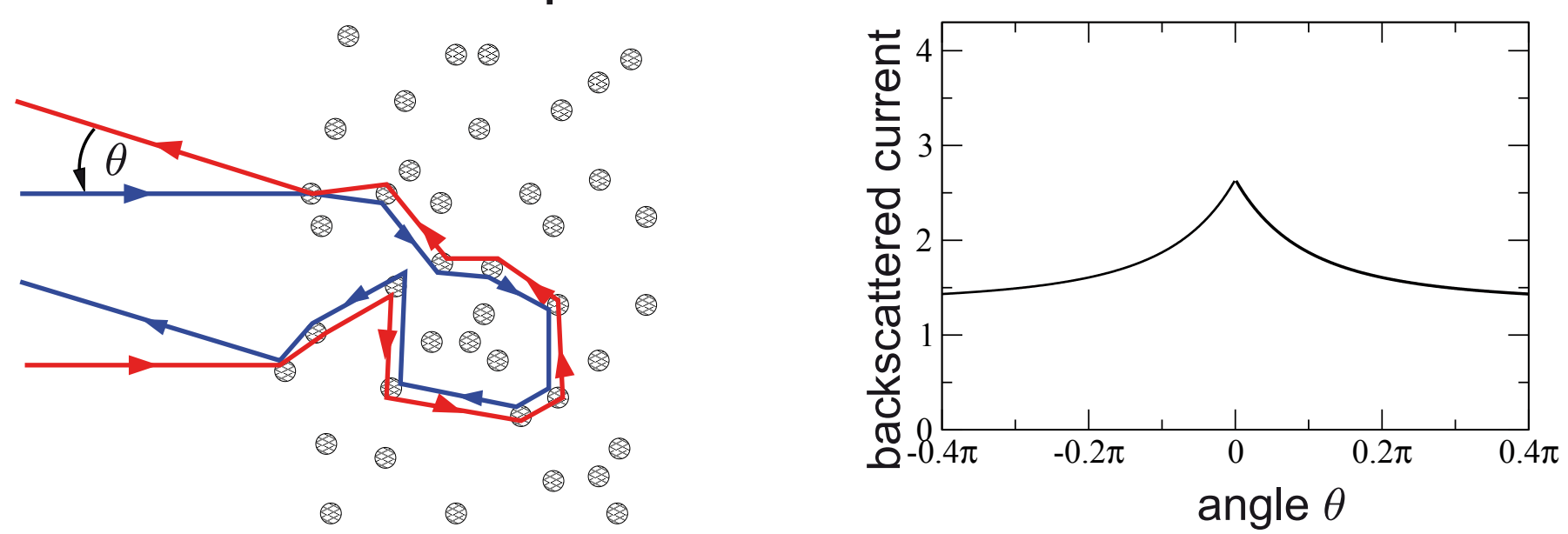

$\rightarrow$ enhanced coherent backscattering of laser light from disordered media

M. P. Van Albada and A. Lagendijk, PRL 55, 2692 (1985)

P.-E. Wolf and G. Maret, PRL 55, 2696 (1985) 


\section{Weak localization in two-dimensional disorder}

$\rightarrow$ Constructive interference between reflected paths and their time-reversed counterparts
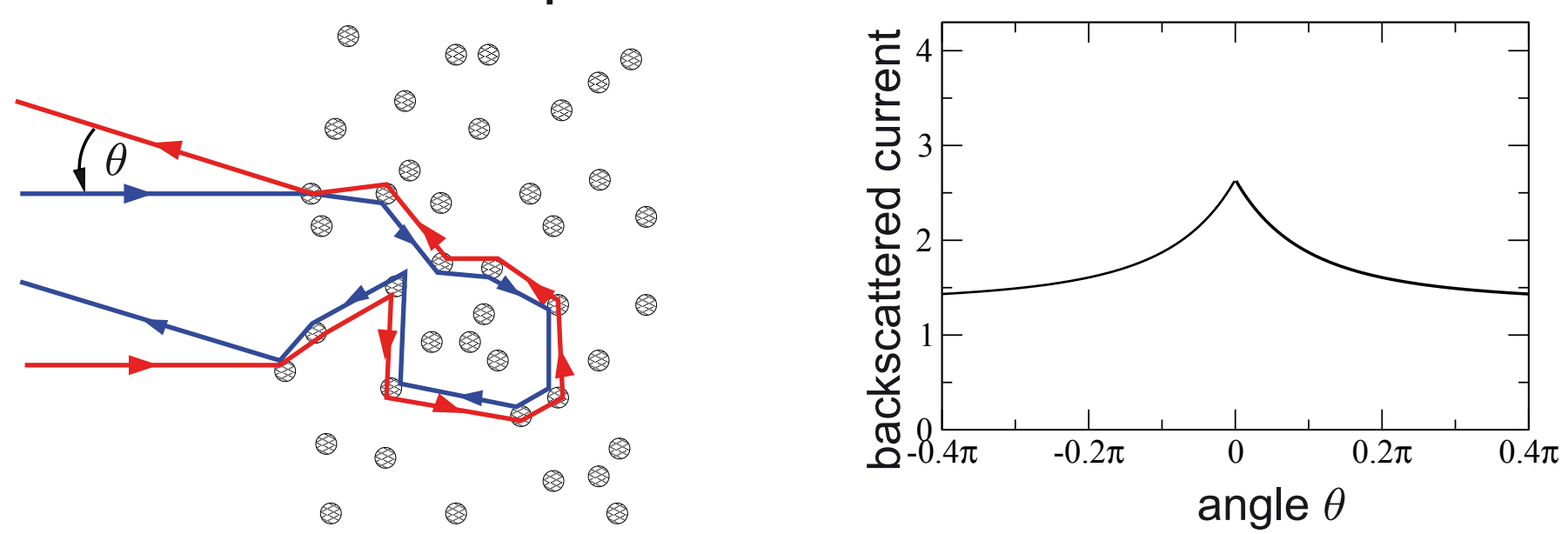

$\rightarrow$ enhanced coherent backscattering of laser light from disordered media

M. P. Van Albada and A. Lagendijk, PRL 55, 2692 (1985)

P.-E. Wolf and G. Maret, PRL 55, 2696 (1985) 


\section{Transport of condensates through 2D disorder}

periodic boundary conditions
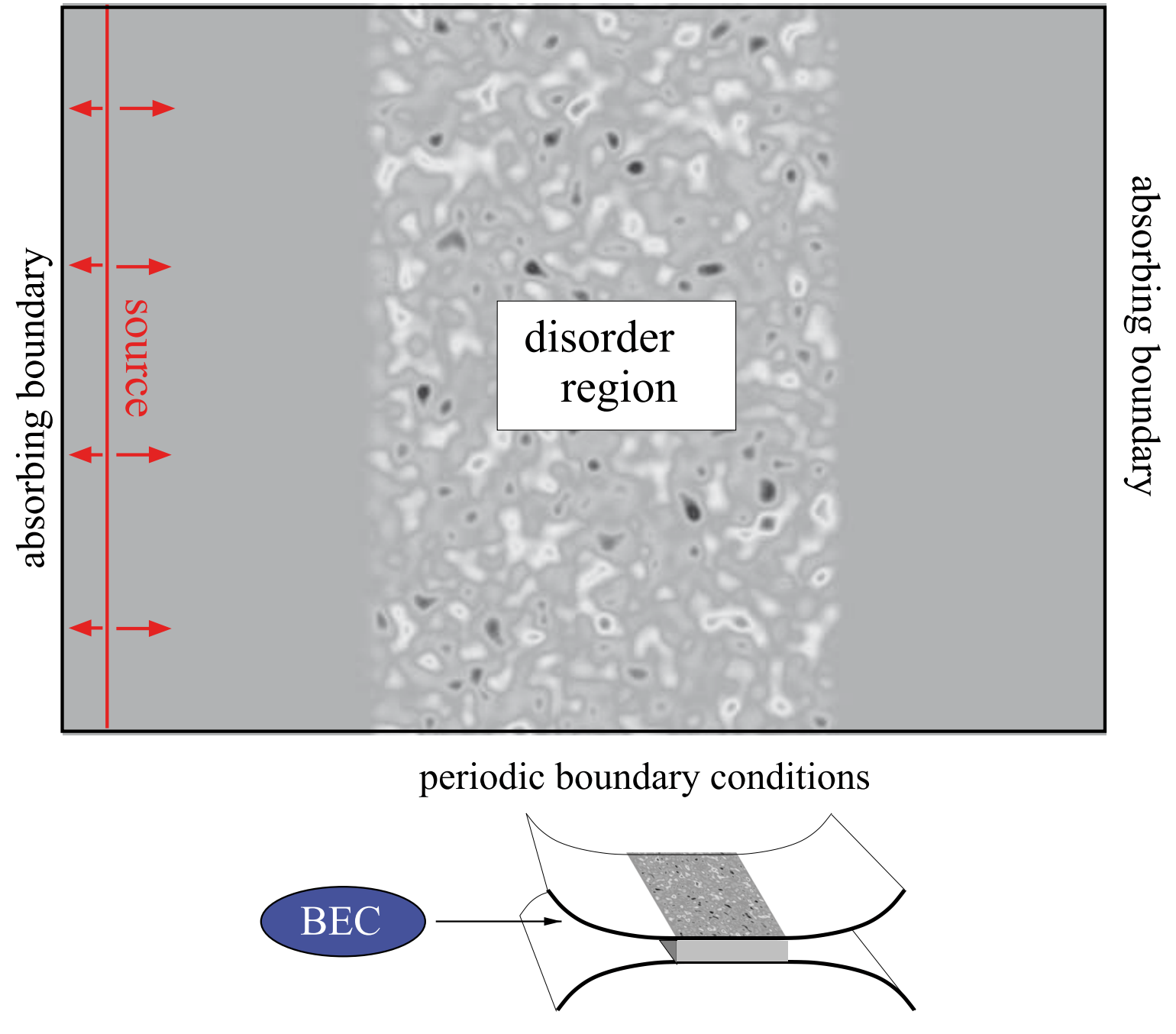


\section{Stationary scattering state of the condensate}

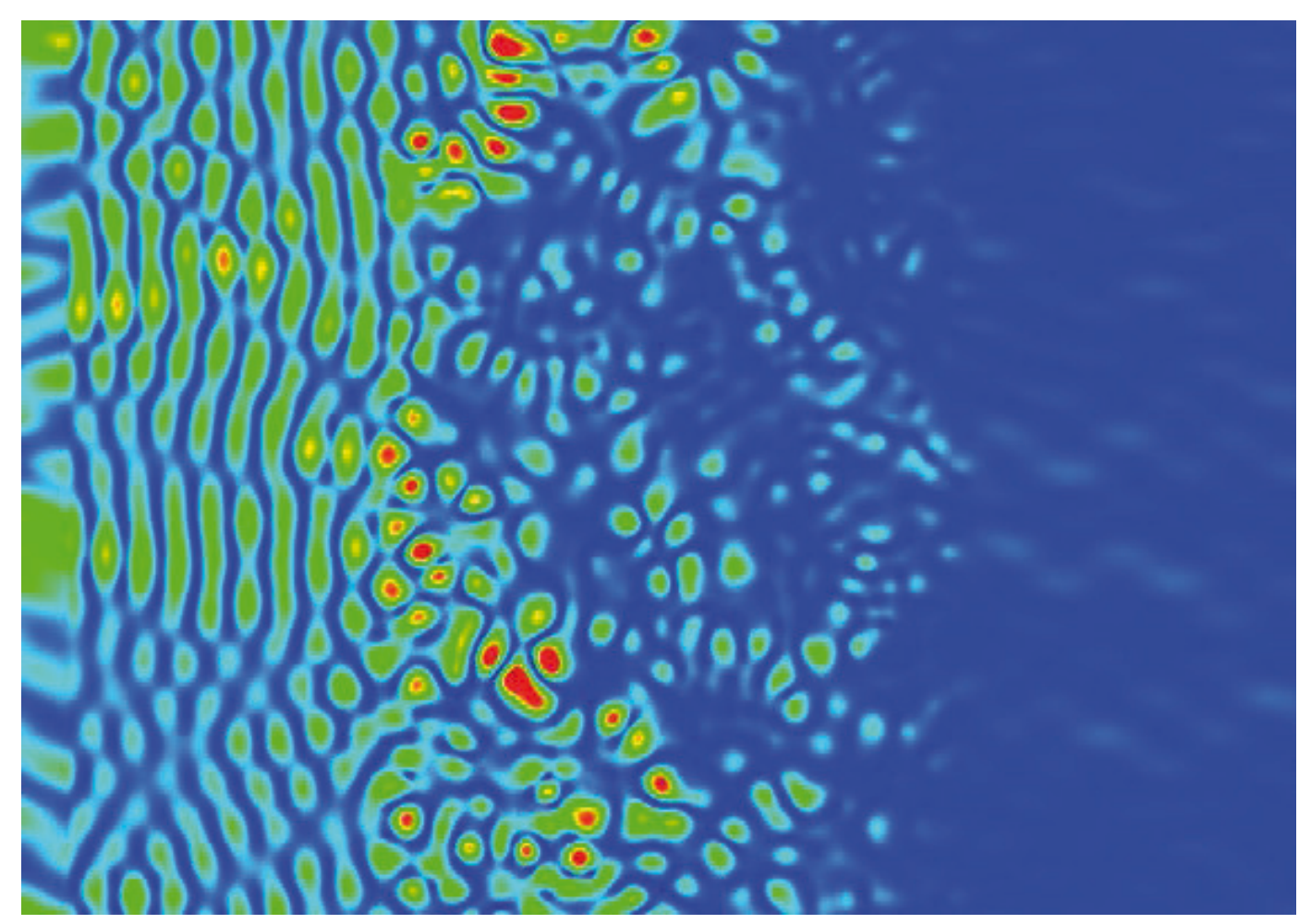




\section{Coherent backscattering of the condensate}

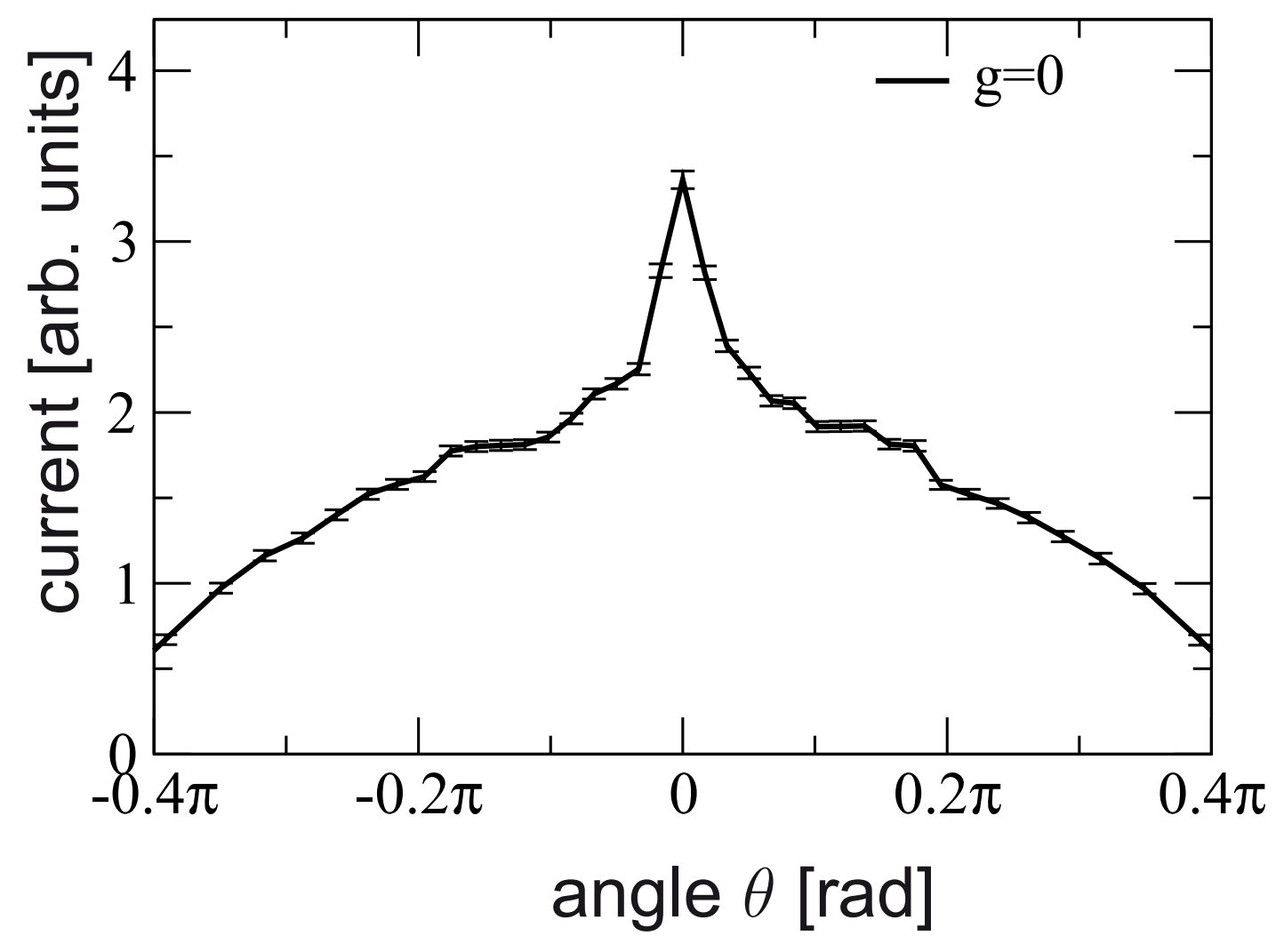




\section{Coherent backscattering of the condensate}

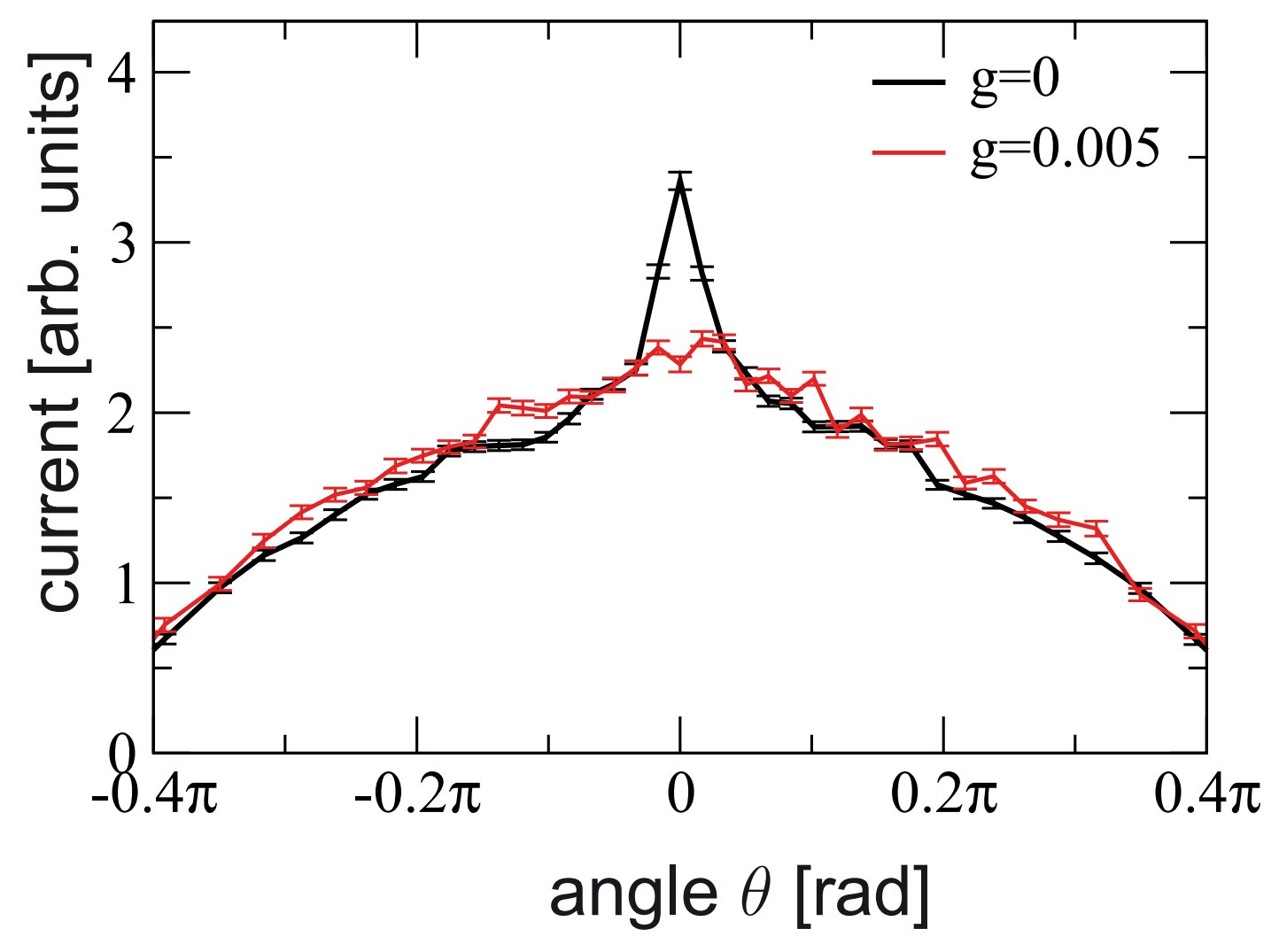




\section{Coherent backscattering of the condensate}

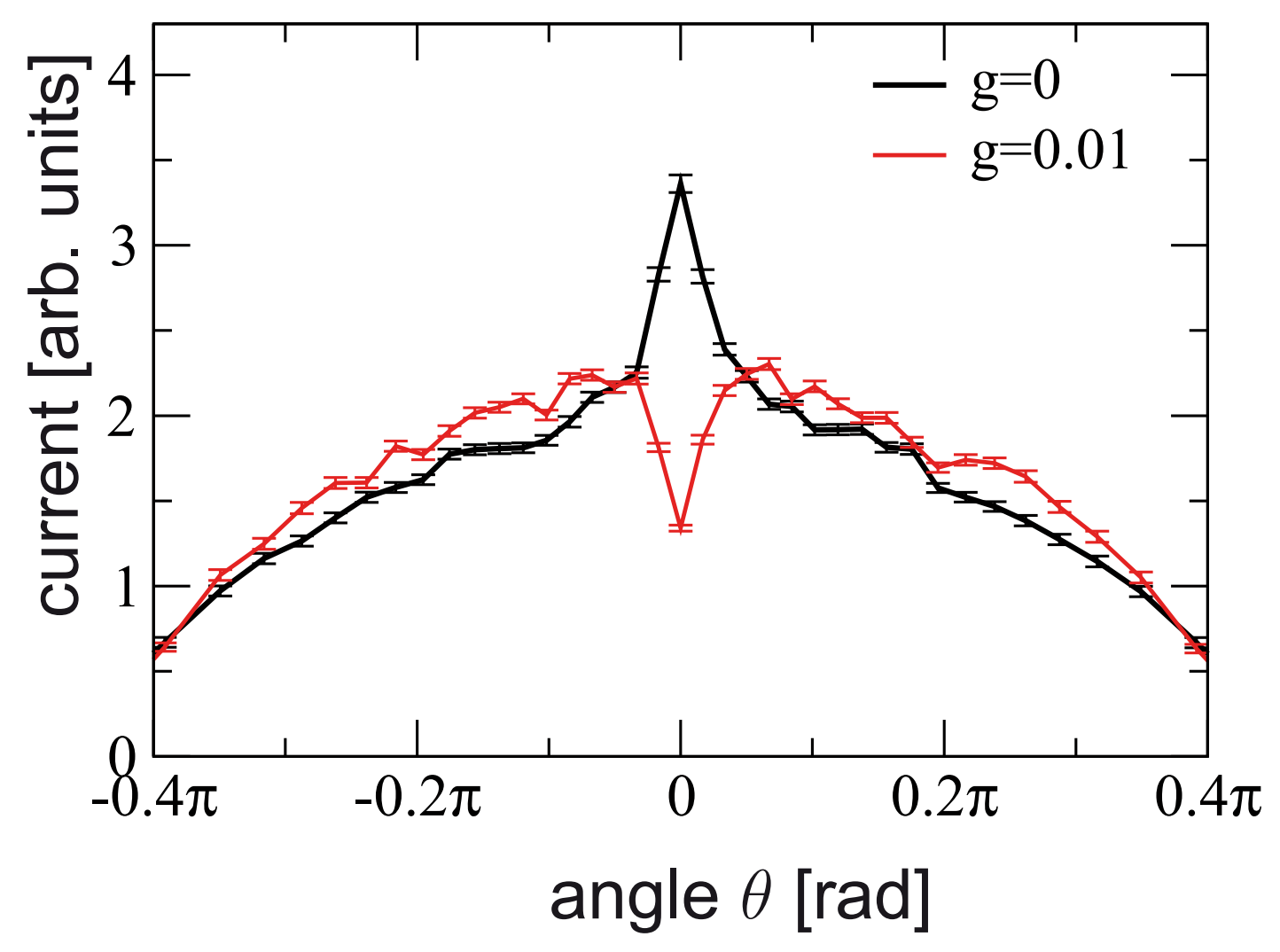

$\longrightarrow$ inverted cone in presence of finite interaction: crossover from constructive to destructive interference

M. Hartung, T. Wellens, C. A. Müller, K. Richter, P.S., PRL 101, 020603 (2008) 


\section{Summary}

- guided atom lasers for studying quasi-stationary transport of coherent bosonic matter waves

- extended scattering potentials and interaction may lead to a loss of coherence

$\rightarrow$ breakdown of Anderson localization

- interaction-induced inversion of coherent backscattering 


\section{Healing length}

Q: The healing length is much larger than any other relevant length scale. Doesn't this invalidate the applicability of the Gross-Pitaevskii equation? 


\section{Healing length}

Q: The healing length is much larger than any other relevant length scale. Doesn't this invalidate the applicability of the Gross-Pitaevskii equation?

A: In a closed 1D system yes. But we have here a scattering process in an open system with a finite extent of the interaction region. The large healing length should therefore not be a relevant length scale. 


\section{"Roton"-like excitations in 1D}

Q: What about excitations along the second Lieb-Liniger branch?

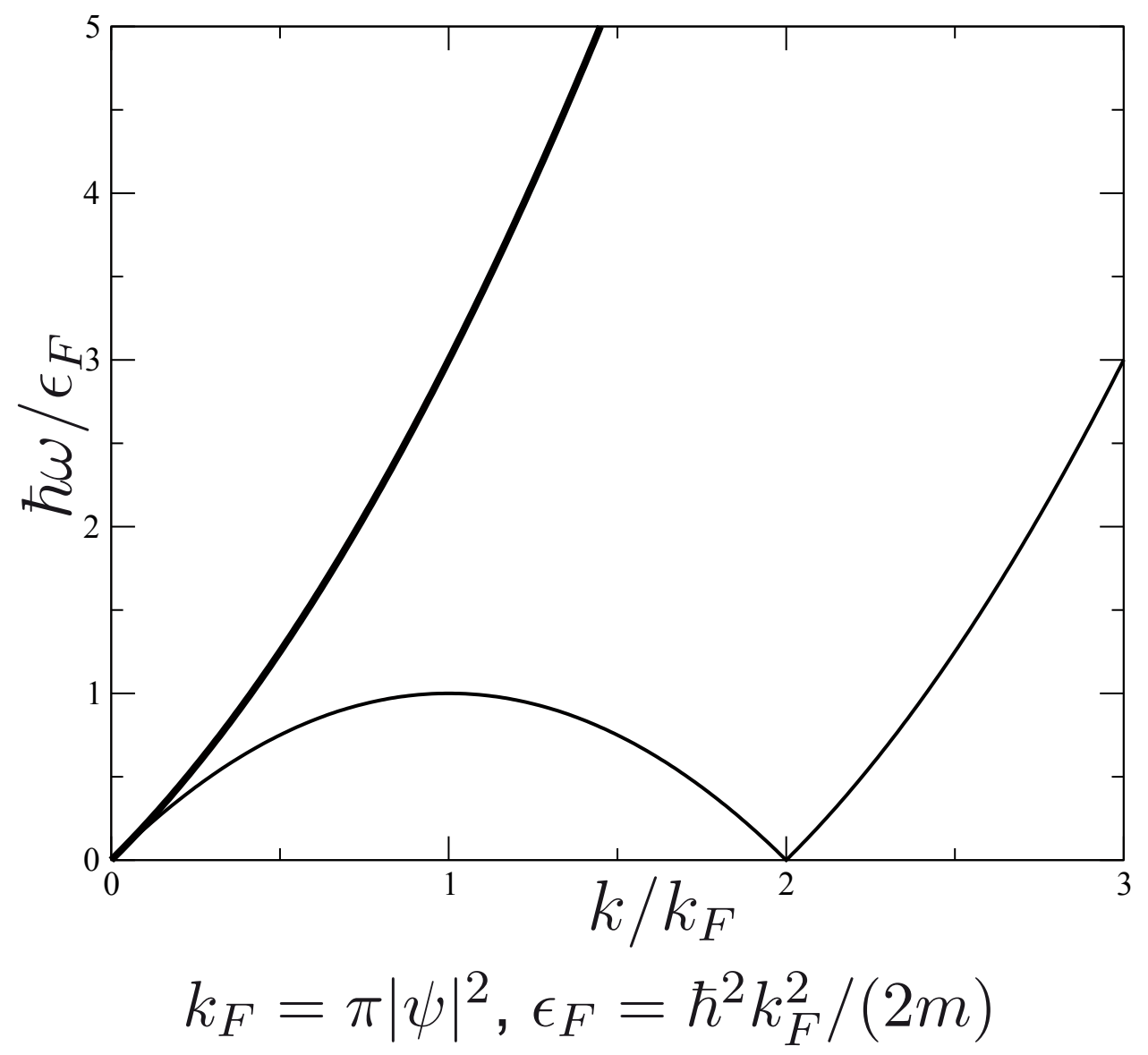




\section{"Roton"-like excitations in 1D}

Q: What about excitations along the second Lieb-Liniger branch?

A: Such excitations cannot be properly accounted for by HFB studies. In closed systems, however, they do not seem to play an important role in the structure factor.

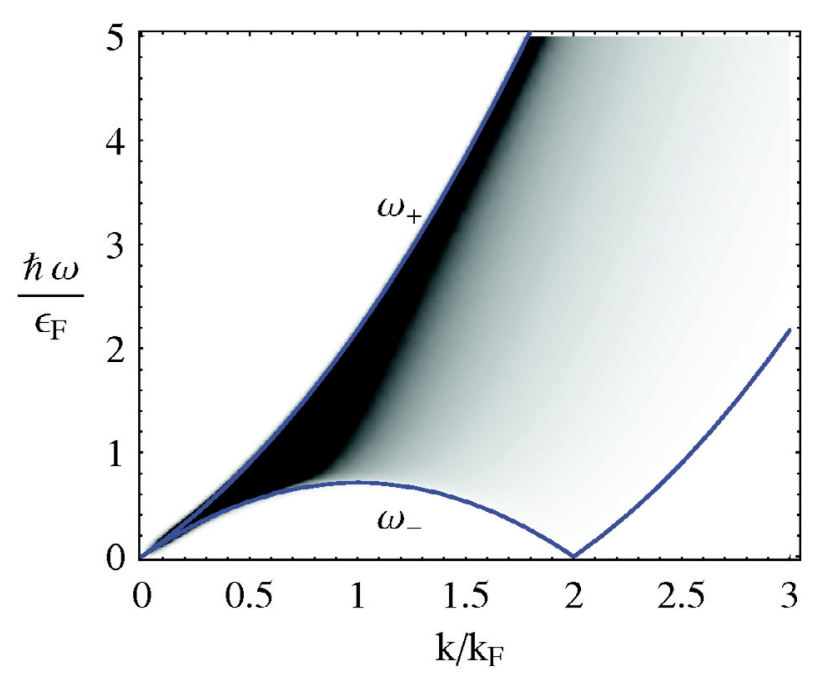

A. Y. Cherny and J. Brand, PRA 79, 043607 (2009)

J.-S. Caux and P. Calabrese, PRA 74, 031605 (2006) 


\section{Infinitely long disorder regions}

Q: What happens for infinitely long 1D disorder regions? Is it possible to have stationary transport (with $100 \%$ reflection) for finite interaction strengths $g>0$ ? 


\section{Infinitely long disorder regions}

Q: What happens for infinitely long 1D disorder regions? Is it possible to have stationary transport (with $100 \%$ reflection) for finite interaction strengths $g>0$ ?

A: We did not attempt to explore this issue with our means. More careful studies M. Johansson et al., EPL 8610009 indicate that the answer is yes. 